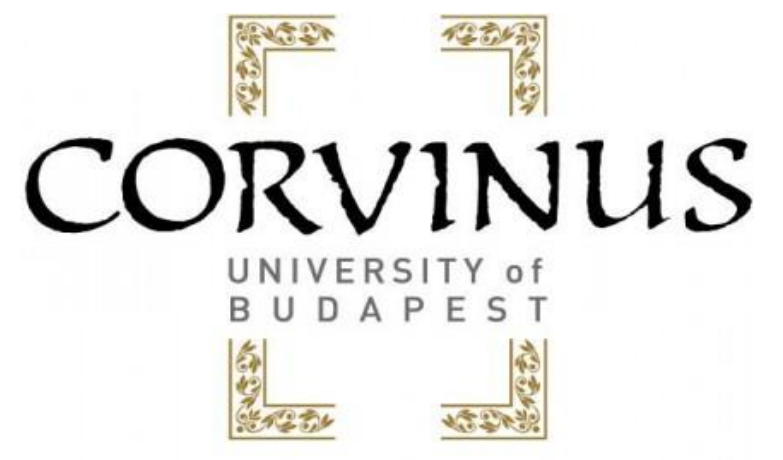

\title{
The Framework of a Real-time Patient Monitoring System
}

Besnik Qehaja

Supervisor:

Dr. András Gábor CSc.

Budapest, (C Besnik Qehaja 


\section{Content}

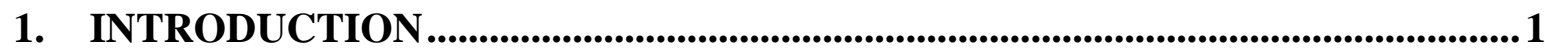

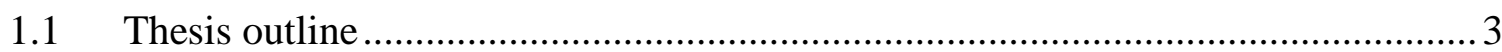

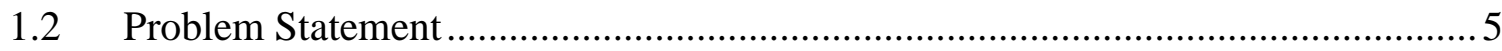

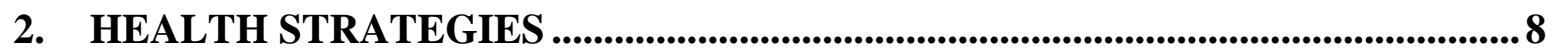

$2.1 \quad$ World Health Organization (WHO).............................................................. 8

2.2 World Health Organization and Europe …………............................................ 8

2.3 Health Strategy of Western Balkan Countries ...................................................... 9

2.4 Kosovo Health Strategy ………………………........................................ 12

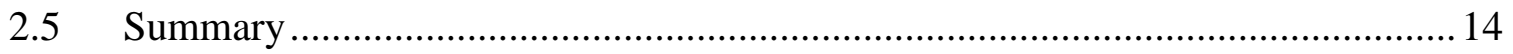

3. Organization of Healthcare ............................................................................................15

3.1 General Practitioner (Family Doctor) ……………............................................... 15

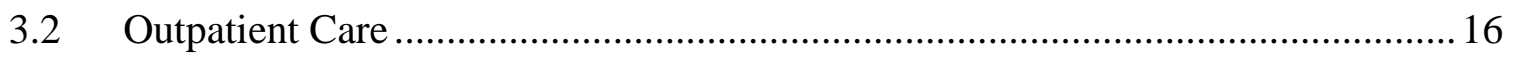

3.3 Tertiary Level Health Institutions ........................................................................ 16

$3.4 \quad$ Homecare - Family Care .............................................................................. 16

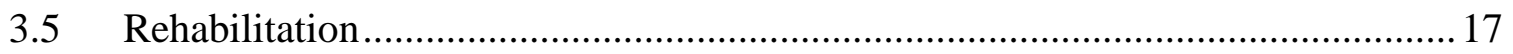

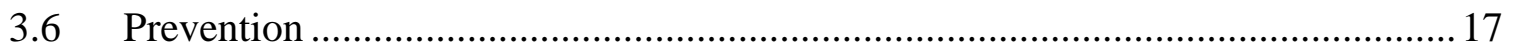

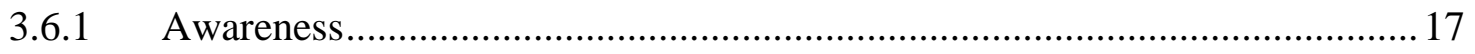

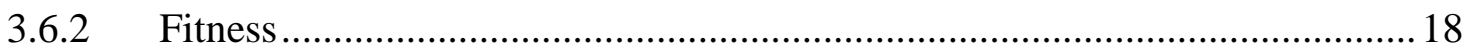

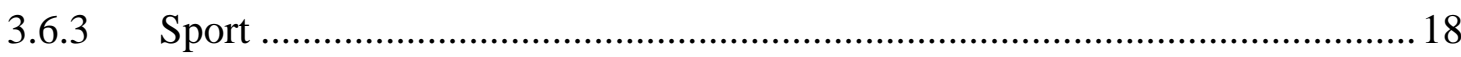

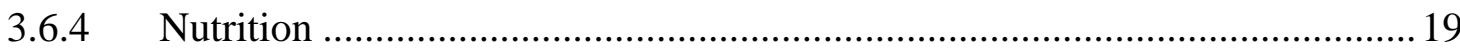

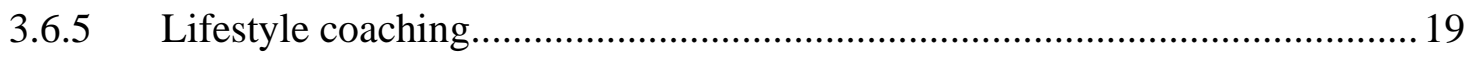

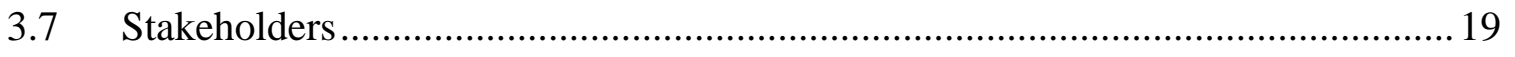

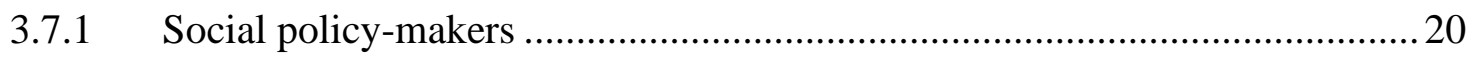

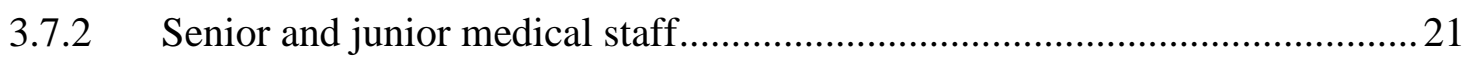




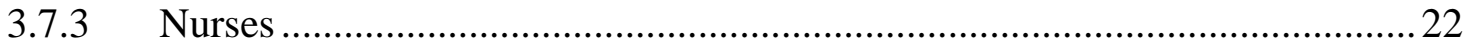

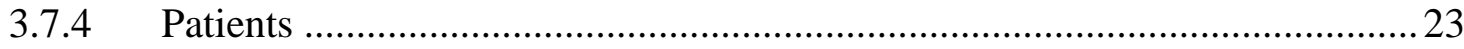

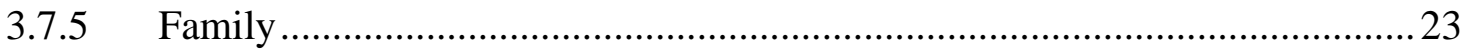

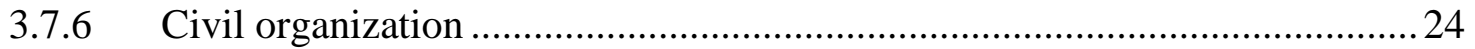

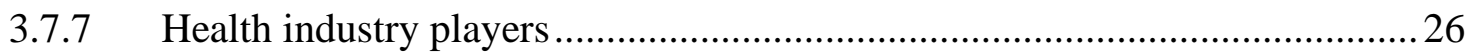

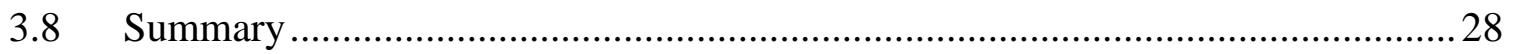

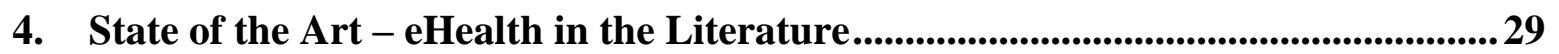

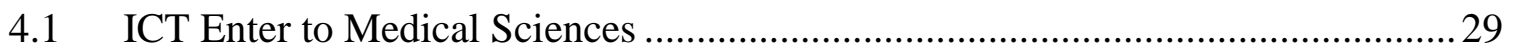

4.2 Digitalization in Healthcare ……………………............................................. 30

4.3 Popularization of ICT in Medical Treatment........................................................ 31

4.3.1 Digitalization in diagnosis and treatment .................................................... 31

4.3.2 ICT Enter into the treatment of patients …………………………………..... 32

4.4 Challenges of ICT in Medical Treatment …………............................................. 35

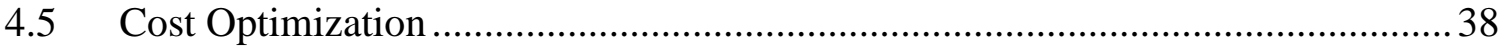

4.5.1 Prevention of disease and early detection through eHealth.............................40

4.6 ICT Infrastructure for eHealth Implementation .....................................................41

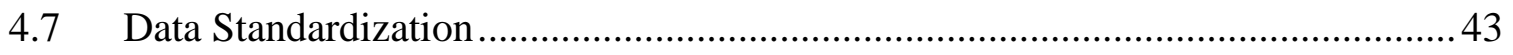

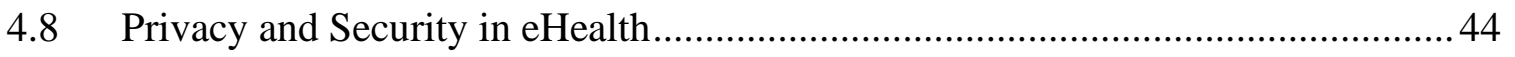

4.9 Types of Hardware Used for eHealth Systems …………………………............46

4.10 Best Practice and Relevant Projects................................................................

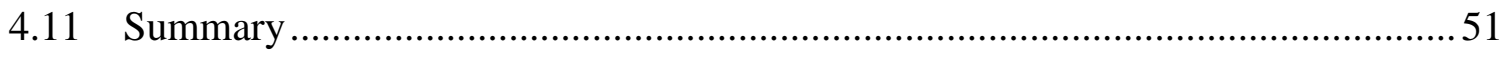

5. Role of Monitoring in eHealth..................................................................................................55

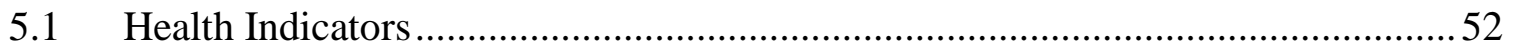

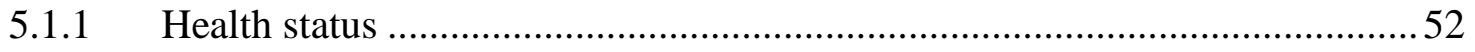

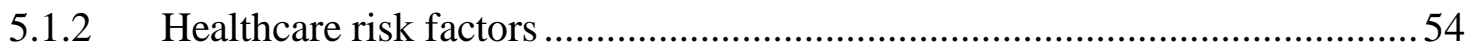


5.1.3 Effectiveness: quality of care and patient experience ................................54

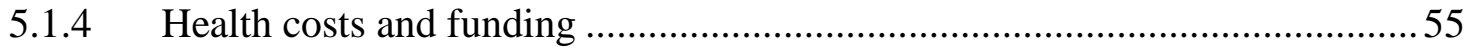

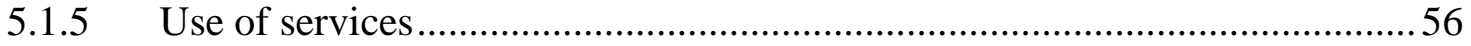

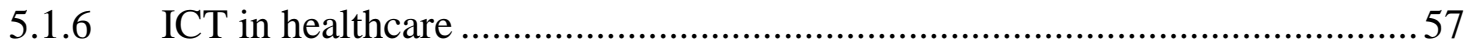

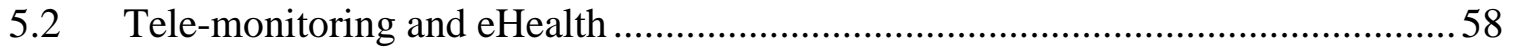

5.2.1 Advantages of Tele-monitoring ..........................................................59

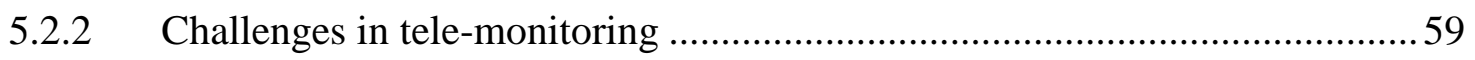

5.3 Implementation: best/good/bad practices ................................................... 61

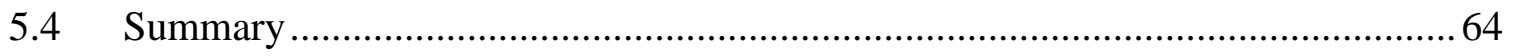

6. Problem Definitions - Research Questions........................................................66

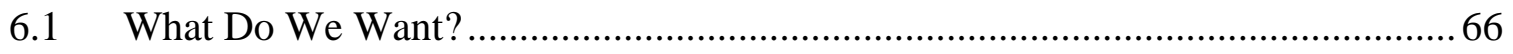

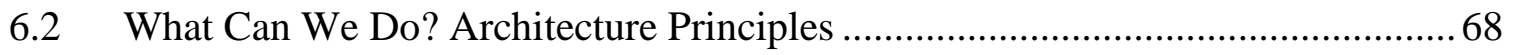

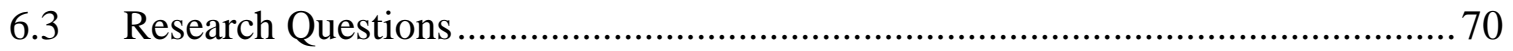

7. e-Health Enterprise Architecture ........................................................................ 72

7.1 Why Enterprise Architecture Approach?................................................ 72

7.2 Current Situation of Healthcare Information Handling in Kosovo....................... 74

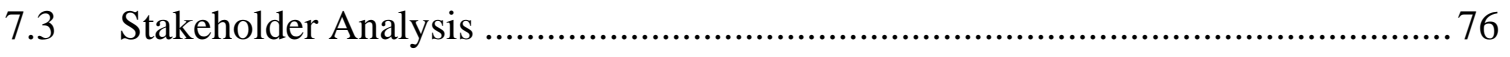

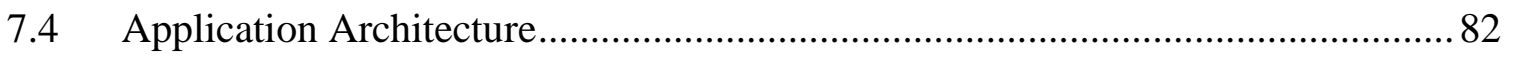

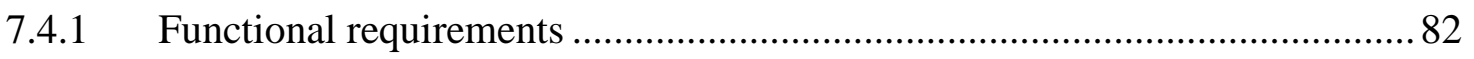

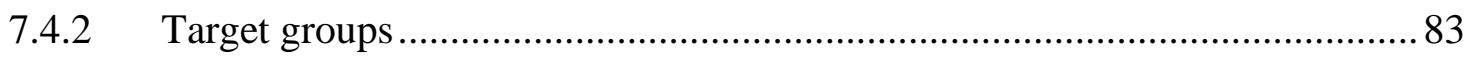

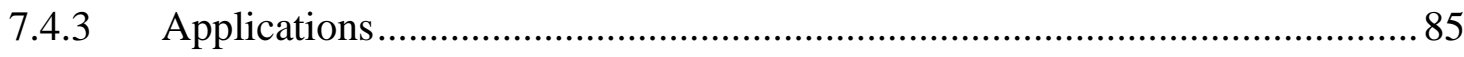

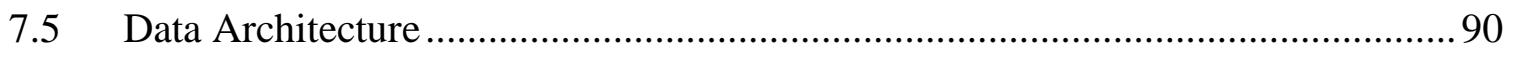

7.5.1 Data analytics in the eHealth system ...................................................95

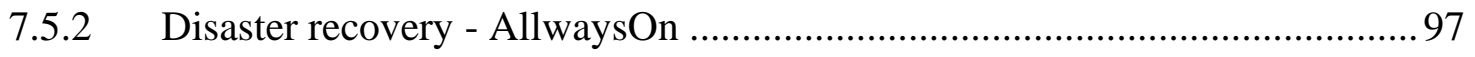

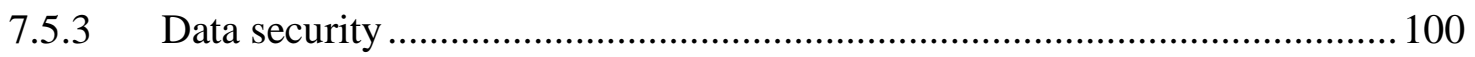




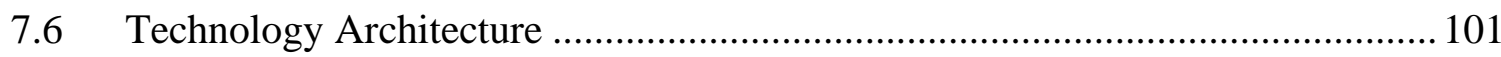

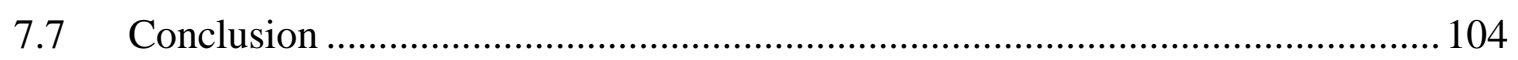

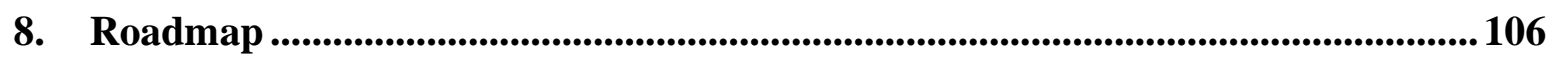

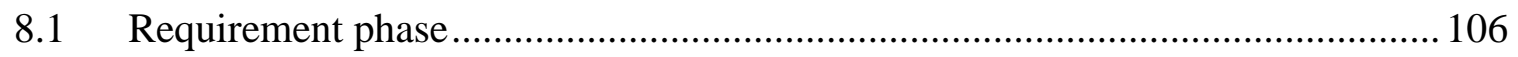

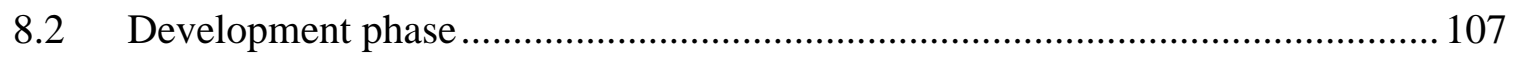

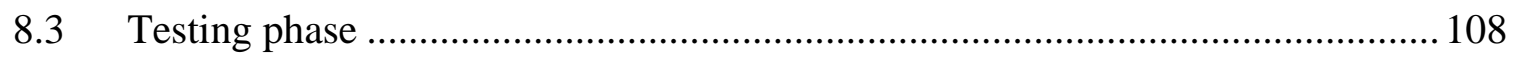

9. Implementation Strategy ………...............................................................................................111

9.1 Preliminary and Planning Tasks .................................................................... 111

9.2 Implementation Phase ……………………………...................................... 112

9.3 Stakeholders' Awareness and Motivation ……………………………...............113

9.3.1 Motivation and awareness raising of citizens .............................................. 113

9.3.2 Encouragement of insurance organization …………………..................... 114

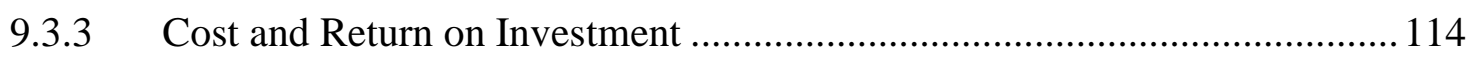

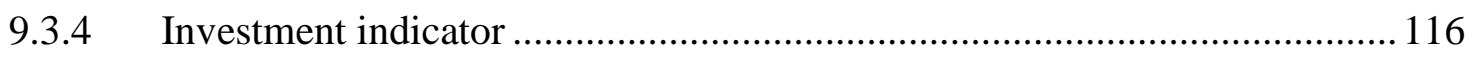

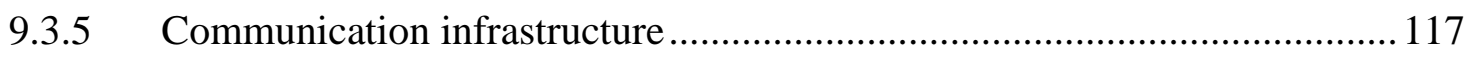

10. Research Methodology and Interview Questions.............................................................118

10.1 Research Methodology ................................................................................. 118

10.2 Selecting Target Groups and the Planning the Interviews ................................... 119

10.2.1 Interviews with medical personnel ........................................................... 120

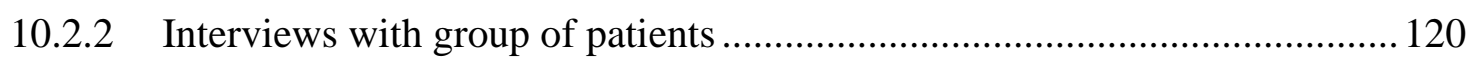

10.2.3 Interviews with ICT experts ................................................................... 121

11. Validation ....................................................................................................................................... 121

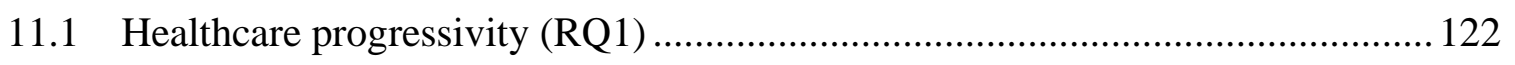

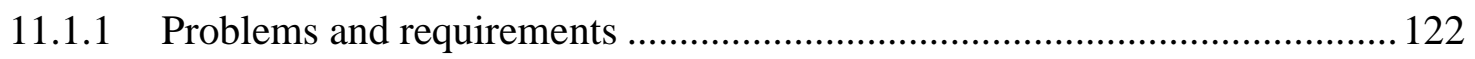

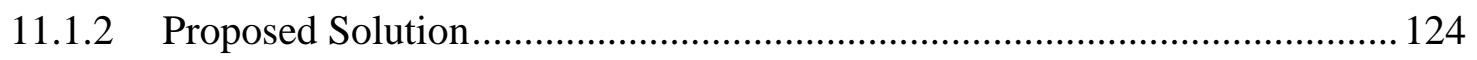




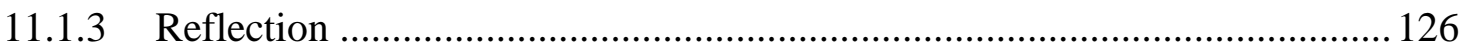

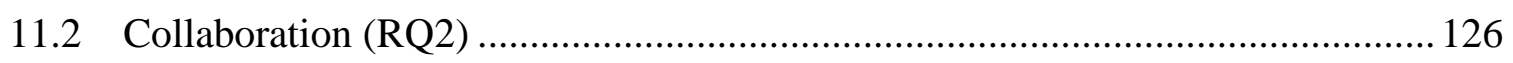

11.2.1 Problems and requirements .................................................................. 127

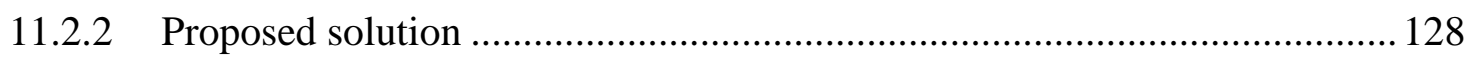

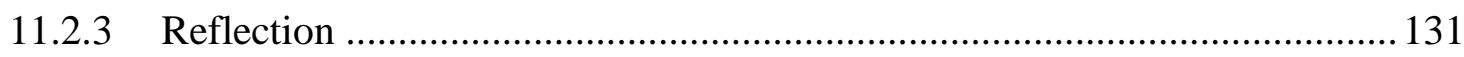

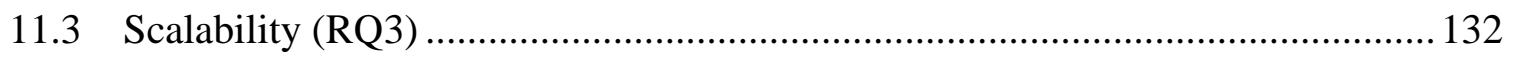

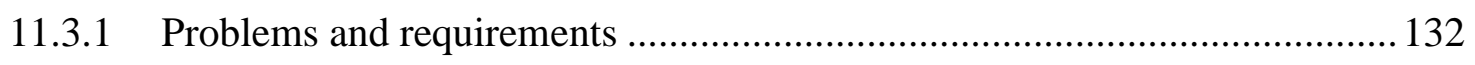

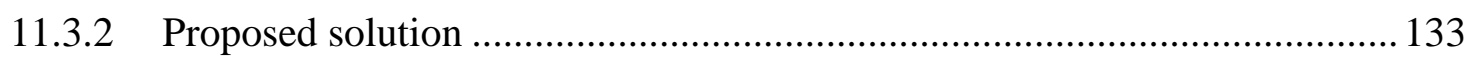

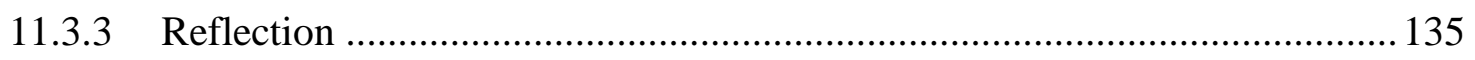

12. Conclusion and Future Direction ......................................................................................136

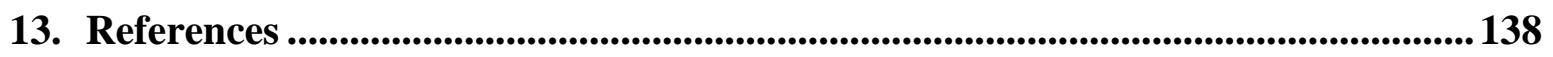

14. Appendix 1: Planning Interview Questions and Target Groups .............................152

14.1 Interview Questions for Medical Doctors........................................................... 152

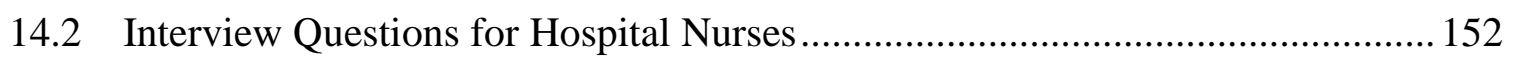

14.3 Interview Questions for Hospital Managers …………………………………..... 153

14.4 Interview Questions for Patients with Chronic Diseases ....................................... 154

14.5 Interview Questions for Patients with Acute Interventions .................................. 154

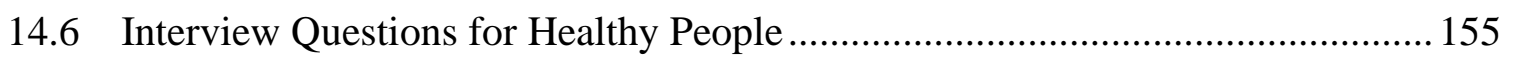

14.7 Interview Questions for Information System Expert ............................................ 155

14.8 Interview Questions for Software Architect Expert …………............................. 156

14.9 Interview Questions for Database Expert ……………………………………..... 156

14.10 Interview Questions for Telecommunication Expert ......................................... 157

14.11 Interview Questions for Cyber Security Expert …………………………….... 157

15. Appendix 2: Summary of the interviews..........................................................................158

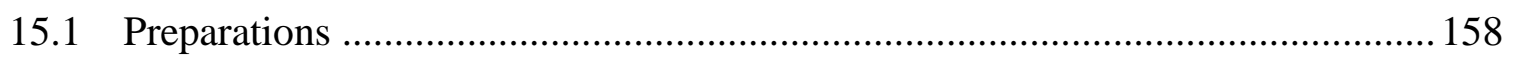




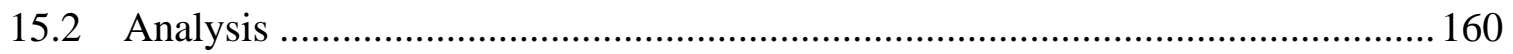

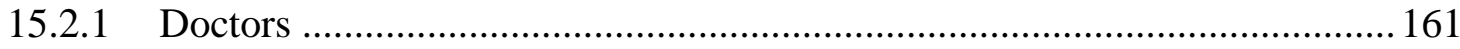

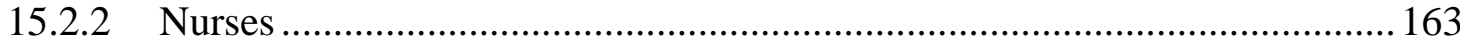

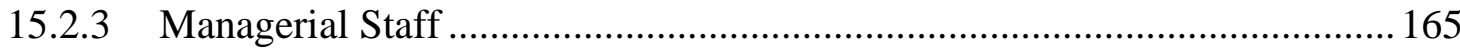

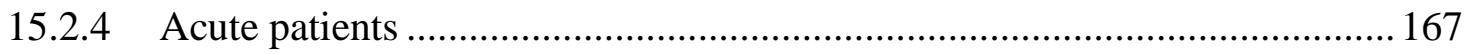

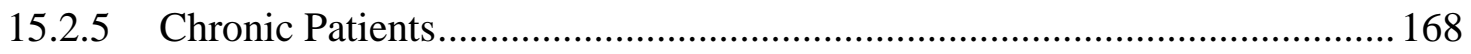

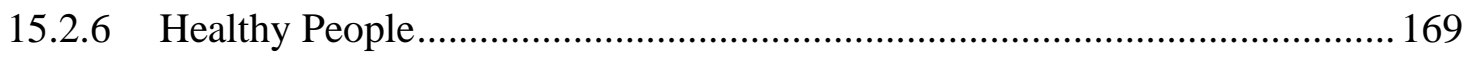

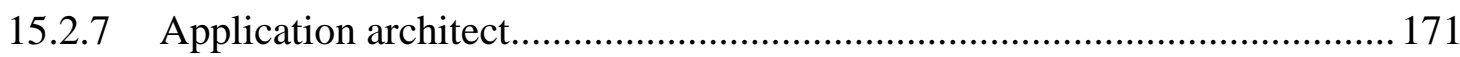

15.2.8 Database (Data Science) Expert …………………………………............ 172

15.2.9 Network communication expert ............................................................. 173

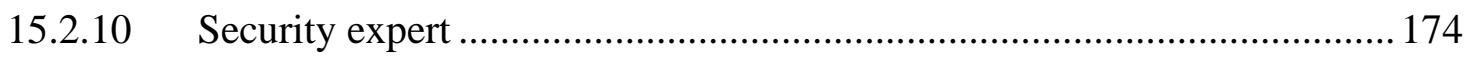

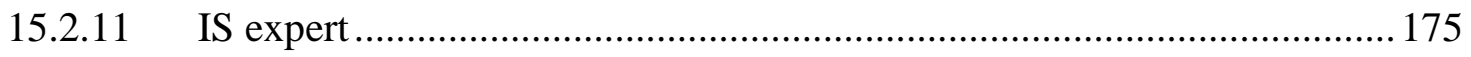

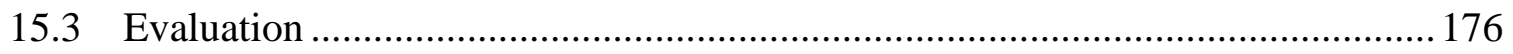

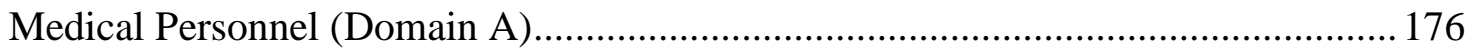

Patients (Domain B) .............................................................................................. 177

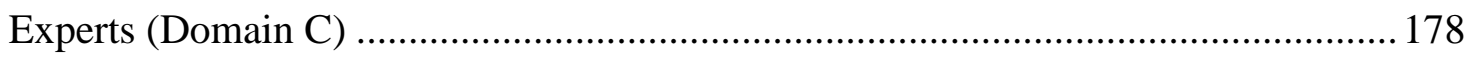

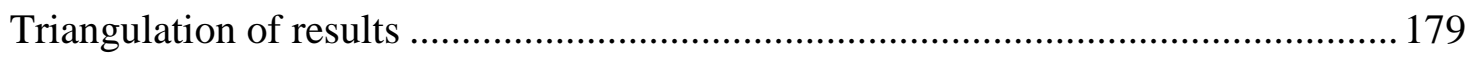

16. Appendix 3: Technology Architecture Repository .........................................................180

16.1 Network WAN and LAN architecture ……………........................................ 180

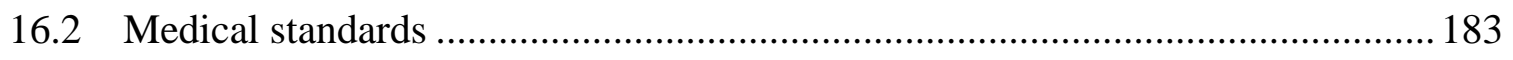

16.3 Communication Security ………………………........................................ 184

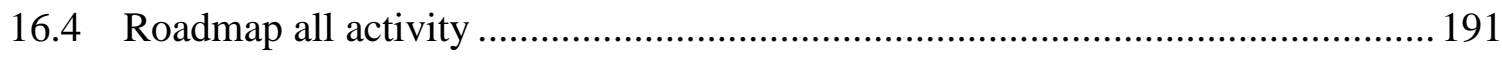




\section{List of Figures}

FIGURE 1. STAKEHOLDER STRUCTURE (CAPOLONGO, 2015) ...................................... 20

FIGURE 2. PATIENT BENEFITS THROUGH THE ICT (ALBAHRI, 2018) ............................ 34

FIGURE 3. CHALLENGES IN EHEALTH SYSTEM (ALBAHRI, 2018)................................ 37

FIGURE 4. STRUCTURE OF MAIN SYSTEMS AND SERVICES IN THE UK (HMSO, 2009)49

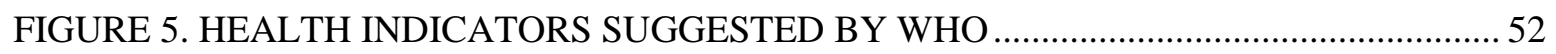

FIGURE 6. MAIN CAUSES OF MORTALITY AMONG WOMEN AND MEN IN EU

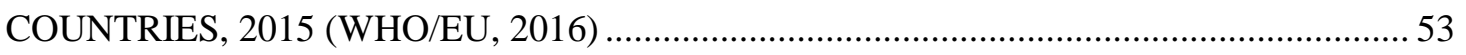

FIGURE 7. LEADING CAUSES OF PREVENTABLE AND AMENABLE MORTALITY IN THE

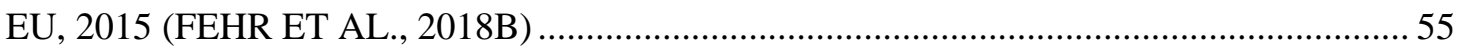

FIGURE 8. HEALTH EXPENDITURE AS A SHARE OF GDP, 2017 (FEHR ET AL., 2018B) .. 56

FIGURE 9. COVERAGE OF HEALTHCARE SERVICES AND PRIVATE HEALTH

INSURANCE FOR 2016 IN EU \& WESTERN BALKAN COUNTRIES .............................57

FIGURE 10. PERCENTAGE OF USE OF ELECTRONIC HEALTH DATA FROM 2012 TO 2016

FIGURE 11. THE MAIN INDICATORS AND BARRIERS IN THE IMPLEMENTATION OF

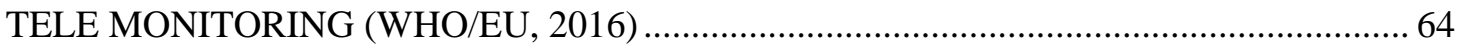

FIGURE 12. INTEROPERABILITY IN THE ARCHITECTURE VISION ................................. 73

FIGURE 13. STAKEHOLDER INTERACTION IN HEALTHCARE .......................................... 77

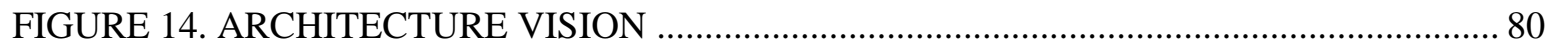

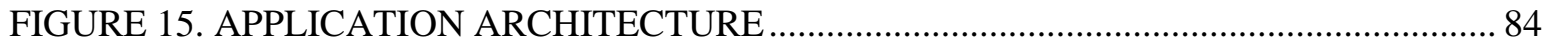

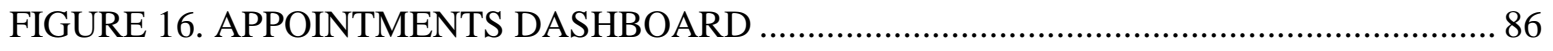

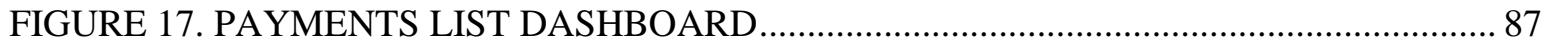

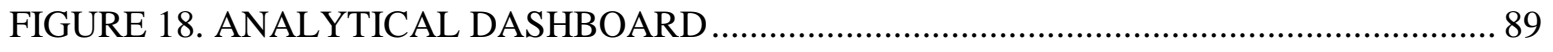

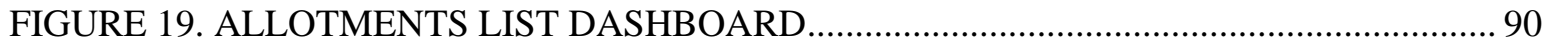

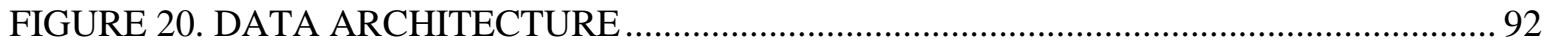


FIGURE 21. DATABASE RELATION STRUCTURE …........................................................ 95

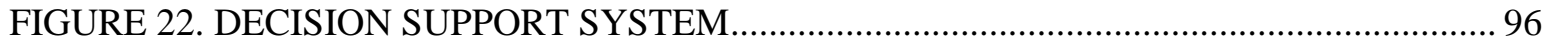

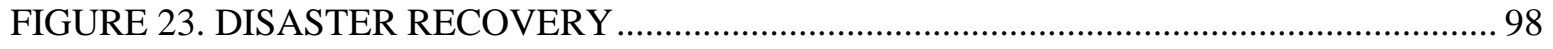

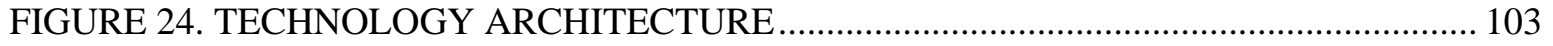

FIGURE 25. ROAD MAP ACTIVITY IMPLEMENTATION (PART ONE) ............................. 109

FIGURE 26. ROAD MAP ACTIVITY IMPLEMENTATION (PART TWO) …......................... 110

FIGURE 27. ROAD MAP ACTIVITY IMPLEMENTATION (PART THREE) .......................... 110

FIGURE 28. FROM PROBLEM TO SOLUTION - VALIDATION …...................................... 122

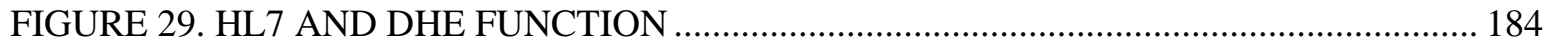

TABLE 1. KEY PERFORMANCE INDICATORS FOR EHEALTH ......................................... 116

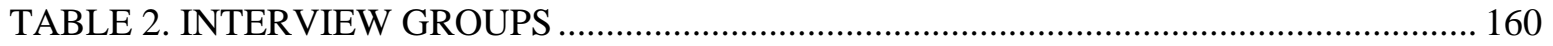




\section{Acronyms}

\begin{tabular}{|l|l|}
\hline AI & Artificial Intelligence \\
\hline API & Application Programming Interface \\
\hline CA & Certification Authority \\
\hline CEF & Cisco Express Forwarding \\
\hline CEN TC251 & Technical Committee 251 \\
\hline COBRA & Common Object Request Broker Architecture \\
\hline CSA & Canadian Standards Association \\
\hline DCOM & Distributed Component Object Model \\
\hline DHE & Distributed Healthcare Environment \\
\hline EHR & Electronic Health Record \\
\hline EHR & electronic health record \\
\hline EMR & electronic medical record \\
\hline ERP & electronic patient record \\
\hline GP & General Practice \\
\hline GPS & Global Positioning System \\
\hline HIE & Health Information Exchange \\
\hline HIS & Health information system \\
\hline HL7 & Health Level Seven \\
\hline HTML & Hyper Text Markup Language \\
\hline HTTPS & Hypertext Transfer Protocol Secure \\
\hline IBM & International Business Machines \\
\hline ICD & International Classification of Diseases \\
\hline ICD-9-CM & ICD ninth revision, Clinical Modification \\
\hline ICPC & International Classification of Primary Care \\
\hline ICR & International Clubfoot Registry \\
\hline ICT & Information and Communications Technology \\
\hline IEEE & Institute of Electrical and Electronics Engineers \\
\hline IHE & Integrating the Healthcare Enterprise \\
\hline IMIA & International Medical Informatics Association \\
\hline IoT & The Internet of Things \\
\hline ISO & International Organization for Standardization \\
\hline LAN & Local Area Network \\
\hline MoH & Ministry of Health \\
\hline$M Q T T$ & Machine-to-machine \\
\hline N3 & The National Network \\
\hline
\end{tabular}




\begin{tabular}{|l|l|} 
NHS & National Healthcare Service \\
\hline PDA & Personal Digital Assistant \\
\hline PHC & Primary Health Centre \\
\hline PHR & Professional in Human Resources \\
\hline PKI & Public Key Management \\
\hline QoS & Quality of Service \\
\hline SAML & Security Assertion Markup Language \\
\hline SHA-1 & Secure Hash Algorithm 1 \\
\hline SNOMED & Systematized Nomenclature of Medicine \\
\hline SOA & Service-Oriented Architecture \\
\hline SSL & Secure Sockets Layer \\
\hline TCP/IP & Transmission Control Protocol/Internet Protocol \\
\hline TLS & Transport Layer Security \\
\hline UML & Unified Modeling Language \\
\hline VPN & Virtual Private network \\
\hline WBAN & Wireless Body Area Network \\
\hline WHO & World Health Organization \\
\hline XML & XML - Extensible Markup Language \\
\hline & \\
\hline
\end{tabular}




\section{ABSTRACT}

The health system is improved when the information system addresses national demands and problems in Kosovo. The progress of healthcare delivery relies on good decision-making in the implementation of the long-term strategy involving all stakeholders in the health system. Best practices, challenges and lessons learned in health system implementation in developed countries are guidelines for developing information systems in healthcare. The interoperability of the eHealth system operates only when the data architecture allows to transmit information between two or more systems allowing one another to process the information in an independent manner and with a single standard. The main objective of health information systems is not to gain information but to improve actions related to providing patient service and improving processes in hospitals.

Therefore, this research is to propose a strategy for the implementation of the health system where the objective is to improve the current situation in healthcare. The thesis proposal will focus on electronic healthcare system strategy and its implementation. A system framework will enable us to understand the daily needs of people with illnesses in order to develop tailored ICT solutions, which can help them, maintain or improve their health status and wellbeing during the time. The cornerstone is the monitoring of the most common diseases in the country, which may have a negative impact on patients' lives. The proposal follows the enterprise architecture principles and tries to set up an implementation roadmap. The functioning of the information system architecture is based on the results of interviews medical staff, patients and ICT experts, who have expressed their opinion on the real functioning of the architecture in accordance with the problems and requirements of users of this system, and in particular, target groups this dissertation. The specificity of this research is that it specifically addresses the monitoring of three groups of patients such as: Acute Patients, Chronic Patients and Healthy people. The results of this research highlight that the digitalization of the health system is indispensable for improving the current state of affairs in both health and administrative terms. 


\section{INTRODUCTION}

Nowadays, the widespread use of Information and Communication Technology (ICT) has covered virtually every aspect of life, including healthcare. The health information system is the creation of a standardized patient data exchange platform, through network infrastructure, hardware and software solutions. Already developing countries are applying electronic health data services, and this is seen as one of the most dominant forms of communication in healthcare institutions. This service is replacing traditional medical services by transforming them into visual electronic form, which data are estimated to be more reliable in the decisionmaking process by medical and management staff in healthcare institutions.

During the research of this thesis, it has been identified that the main problem in developing countries, especially in Kosovo, remains high mortality, where the findings show that mortality has increased especially as a result of chronic illness due to lack of information at the time of appropriate for medical staff and patients. At the same time, there is a high lack of digitalization of processes in the management of medical services, which has directly impact on the health of the population and the performance of the stakeholders, which provide health services. The research has also resulted that Kosovo has failed in many phases.

The national strategy for the implementation of the eHealth system as well as the state of health services in Kosovo remains with traditional paper-based methods.

In reviewing the literature, researchers argued that the implementation of information systems has provided first results in medical services, especially in patient monitoring and administrative processes. The literature in this dissertation has served as a basis and argument and has covered a large number of key indicators in the healthcare systems, starting with the WHO organization's strategy for developed European countries. During the research, the Strategies of developing countries, respectively Western Balkan countries, have been reviewed, analyzing how advanced they have been towards Kosovo and how much they have adhered to the WHO recommendations. Whereas, based on the National audit reports, there have been identified failures in the implementation of the Health System Strategies in Kosovo and beyond. Viewed literature is also used to elaborate all technologies that include the health information system, ranging from application architecture, data architecture, and technology 
architecture, which support information systems in their performance. At the same time, a special focus on this dissertation is given to standards and safety of medical records, which are a priority in a healthcare system.

Within this subject, the objectives and research questions are formulated to respond to the current situation and the demands of all stakeholders in healthcare. While research questions relate to my assumptions and recommendations that are given in the technological aspect where my proposal includes a comprehensive eHealth Implementation Strategy that is in line with the requirements arising from the current situation and in line with the WHO Strategy, for developing countries. The Strategy implementation recommendation is based on the TOGAF framework, which includes all levels, from application architecture, Data architecture, and technology or hardware architecture, as well as describing and visualizing the steps and tasks of implementation starting from Baseline architecture to target-line architecture. I have addressed three research questions, which directly related to the objectives pursued in this research.

- Will the proposed architecture (and how) reduce the level of progressity of healthcare in terms of resource usage and volume of patients?

- Will the proposed architecture improve (and how) the collaboration among the various actors?

- Will the proposed eHealth system (and how) scalable and modularized enough to address the different technical and cultural readiness of various users group?

Therefore, the research questions are based on the current situation and the objectives set below, whereby an Information System Implementation Strategy is proposed, which will respond appropriately to each research question in this dissertation. Also the proposed strategy along with the recommendations and requirements of the target groups interviewed will show the correlation of how the problem is addressed to research questions, where through these research questions will find the answer to the system architecture proposed by me, as well as by treating how related are user requirements during interviews with target groups that will use the proposed health information system. 
Research methodology cover in this study, a combination of three methods is used because of the nature our research questions that require multiple methods to get them answered. Combining methods offers great promise on flexibility of the research, draw strengths from multiple methods, and therefore allow the research to answer questions in a broader way that are not confined to only one method. The objective is to understand the challenges and propose technology-based solutions that can improve the data flow between different levels of healthcare systems. The survey strategy is associated with the deductive approach, and it is an appropriate procedure to answer questions like who, what, where, and how is doing. The questions in the following sections (appendix), give the skeleton of the planned interviews. This research is primarily based on the five regions of the Republic of Kosovo. The data and opinions will be taken from six hospitals, including three well-known international healthcare centers. From each hospital these roles of interviewees will be selected: medical doctor, nurse, hospital manager, patient and experts of ICT .The purpose of data collection via personal interviews is to understand better the existing work conditions of doctors, nurses and administrators, then, through interview questions, learn about their views on digitalization in medical services, collect the specific requirements from the view of their specific role, finally get some input in regard to their willingness accepting digital system outlined in the thesis. At the same time, the questions and recommendations emerged from the interviews serve as a basis for validating the proposal in this dissertation in the technological aspect of implementing the Strategy. The functioning of the information system architecture is based on the results of interviews of ICT experts, who have expressed their opinion on the real functioning of the architecture in accordance with the problems and requirements of users of this system, and in particular, target groups this dissertation.

\subsection{Thesis outline}

The topic structure is divided into 12 chapters, where the scope of the topic extends theoretically and practically based on scientific findings, and the results are interpreted as easily as possible for readers.

Chapter 1 contains an overview description of the topic, briefly describing the entire Dissertation so that readers will have a preliminary concept of the topic as a whole. This 
chapter also describes the problem statement, which elaborates, on the problems to be addressed in this dissertation.

Chapter 2 includes research on implemented health system strategies and their future planning. Also included in this chapter is the World Health Organization strategy (WHO), the Health strategies of states at the international level, strategies in developing countries and especially the Balkan countries. And, in the end, the weaknesses and strengths of the Kosovo Strategy are also discussed, based on the audit reports at the local level of Kosovo, but also at the international level (WHO).

Chapter 3 covers a very important part about the organization of the health system in the Balkan countries, thus identifying all health and non-health stakeholders, where the findings served as information on their behavior / need for interconnection with a digital system.

Chapter 4 covers the reviewed literature, which has served as a basis for evaluating the achievements of eHealth systems, as well as the challenges and achievements for digitizing the health system internationally. The literature also identifies the most used technologies in eHealth systems, as well as the challenges they face so far, especially in the interoperability and communication of eHealth health systems.

Chapter 5 covers the elaboration of key indicators on the functioning and non-functioning of the health system, based on reports generated by Eurostat and WHO. Indicators include reports on social, technological and financial problems. Also at the end of this chapter are case studies of tele monitoring implementation in health systems, identifying the problems, challenges, and best methods of tele monitoring implementation in health systems around the world.

Chapter 6 describes the problem definition by addressing the three research questions of this research, as well as the research objectives through two personal questions on my research: What do we want and what can we do?

Chapter 7 includes the proposal of the eHealth system implementation strategy for real-time patient monitoring, based on the problems, requirements and objectives set. The strategy includes an architecture based on TOGAF framework, including Architecture Vision, application architecture, data architecture, and technology architecture. Each architecture is 
described from a technical point of view and theoretical point of view so that the architecture is as persuasive as possible to the problem and research objectives.

Chapters 8 and 9 includes a presentation of Roadmap and Strategy implementation step by step with all the activities, which are visualized on a chart and are traversed by activities from Baseline Architecture to Target-line Architecture. All stages of the strategy implementation are based on the TOGAF framework using (ADM stages) and also we use a method agile and waterfall depends on the situation.

Chapter 10 includes the research methodology that was conducted during the research, as well as the planning of interviews conducted with stakeholders, in order to validate the research questions and justifies the system proposed by me.

Chapter 11 includes interview results and validation of research questions, thus attempting to elaborate on the findings from stakeholder interviews on how well they are consistent with the proposed system, and through expert interviews we sought to justify the recommended proposal against the requirements of users.

Chapter 12 includes conclusions on the topic and recommendations for the future of digitalization of the health system.

Chapter 13 includes a list of references, while Chapter 14, 15, 16 cover the Appendix includes detailed empirical data for each target group of interviewers, along with the questions formulated for all interviewees in this research. Also supporting material generated from the literature review chapter, and part of our proposal chapter is set out in the appendix.

\subsection{Problem Statement}

During scientific research, reports and other relevant documents related to the health system in Kosovo, it has been evidenced that although after 19 years after the war we still have serious problems regarding access to health services. The increasing scale of mortality is evident every year as a result of healthcare. At the same time, there is a lack of digitalization in work and management processes in hospitals. Besides that, many social indicators witness

for a worrisome situation in this regard. Personally speaking, I have been encouraged and eager to deal with this field, hoping to contribute to improving healthcare by using ICT. The 
current Kosovo system has demonstrated the failure of functioning the healthcare system and the low performance in providing timely, effectively, efficiently and patient-safety secure care. Generally speaking respective instances in Kosovo lack in-depth knowledge regarding e-health care and no one is thinking of a solution for virtual communication between the patient and the doctor. Indeed, as mentioned below, the implementation of the Health Strategy has failed from 2010 to the present day and we still do not have an information system that supports processes in the health system. The whole range of data on the processes and services performed within hospitals and other information about patients is based on paper. This situation causes drawbacks and hampers all stakeholders starting with patients, family members, medical staff, and all participants in health care. The available human and technological resources for public health services are the lowest levels compared to other European countries. Fundraising for investment strategies continues to pose a challenge for us as a people. Obviously, there has been witnessed a lack of properly utilizing existing resources to bring above positive changes in this aspect. Nevertheless, it is worth emphasizing that there is an urging need to improve health services should be improved especially in the treatment of acute and chronic diseases, which are the main indicators of the death of the population. Various age groups, in particular, middle and senior groups, which mainly represent a large part of the population, have limited access to health services and their illness poses a major economic burden for their families and the state in general. Analysis of the health status of the population shows that the number of deaths in 2016-17 ranges to 421.9 deaths per 100,000 inhabitants. Apart from mainly paper-based treatment, the problem remains the lack of qualified healthcare staff who are migrating, and this is an obstacle to covering patient requirements for treatment.

The use of ICT in medical services has made it possible for us as researchers in the field of health care to contribute to acceptable solutions to overcome this situation by using ICT as a key tool for improving health services. Therefore, my intention is to propose a strategy for the implementation of the health system where the objective is to improve the current situation in healthcare. This proposal will mainly focus on recommending an electronic healthcare system strategy implementing. Additional programs for the monitoring and management of comprehensive healthcare surveillance for the three-target group below. A 
system framework will enable us to understand the daily needs of people with illnesses in order to develop tailored ICT solutions, which can help them, maintain or improve their health status and well-being during the time.

\section{PART ONE}




\section{HEALTH STRATEGIES}

\subsection{World Health Organization (WHO)}

World Health Organization, at its international level, has mandated the role of eHealth through two World Health Assembly resolutions: WHA58.28 (2005) on eHealth (4) and WHA66.24 (2013) for eHealth standardization and the interaction of information systems. Through these two resolutions, the organization regulates the role and responsibilities of WHO's contribution to the global eHealth agenda and the needs and requirements of UN member states in WHO. The intention is the proper use of information and communication technologies in order to improve care and increase the level of engagement of patients in their healthcare, the provision of quality health services, and the sustainable financing of healthcare systems and the promotion of universal access (WHO, 2016b).

\subsection{World Health Organization and Europe}

The Action Plan for National eHealth Strategy describes in detail, how WHO has an active role in the approval and implementation of eHealth strategies in European countries, where according to the WHO Europe Strategy (Gjorgjev, Bacanovic, Cicevalieva, Sulevski, \& Grosse-Tebbe, 2006) .European countries have important indications in their eHealth adaptation steps and signals the way forward for new approaches to health and wellbeing around the world. The WHO's main goal under the 2020 Strategy is that in European countries, the national or local health management will be replaced by standardized and unified health systems so that they can be interconnected. European countries through the approval of the WHO 2020 Strategy have been endorsed for two common objectives, the need to improve health for all, and the need to strengthen leadership and participatory governance in healthcare. Through these two objectives, the main goal of the countries in the region is to reduce premature mortality by 2020 , increase life expectancy, reduce health inequality, increase population wellbeing and cover health insurance at the universal level.

Based on the WHO global health survey statistics for (WHO/EU, 2016) European countries have shown tangible progress in the integration of ICT to improve public health and provide health services. However, results show a comprehensive and cross-sectoral approach to the development of eHealth national strategies is recommended to ensure their relevance for all 
stakeholders and to promote joint actions in achieving health objectives. The WHO Survey for European Countries is primarily has 8 key points: eHealth foundations, Electronic Health Records, telehealth, mHealth, eLearning in health, social media in health, health analytics and Big Data in health, as well as Legal frameworks for eHealth. According to results of this survey show $84 \%$ of WHO Member States in the European region have drafted policies aiming to enhance to support their progress in achieving universal health insurance coverage. $70 \%$ of the EU Member States have a national eHealth policy or strategy; $69 \%$ have financial support specifically available for the implementation of eHealth strategy or national policies; It is worth emphasizing that approximately $60 \%$ reported they have a policy or strategy that guides the development of their national SIS. Whereas $89 \%$ provide education or training on the use of ICT and eHealth. As far as e-Learning is concerned, the results show that $71 \%$ (32 member states of the European region) use eLearning to train health professionals. Meanwhile, as far as social media is concerned with regard to the use of social media, the survey results show $91 \%$ of respondents (40 states resp.) reported that individuals and communities use social media to learn about health issues, while 81\% (35 states) reported healthcare organizations use social media to promote health messages as part of health promotion campaigns (Fehr, 2018).

Based on WHO survey results, recommendations are for states to use the WHO's National Health Strategy and integrated the International Telecommunication as a basis for developing their national vision as well as tracking the action plan and monitoring and evaluation frameworks for eHealth. However taking into account WHO poll results, it points out that states have a national eHealth strategy that is supposed to have the elements to attain and implement the Health 2020 policy, which is a key factor in strengthening people-oriented health systems and healthcare capacity public (Fehr et al., 2018a), (WHO, 2016a).

\subsection{Health Strategy of Western Balkan Countries}

Compared to the developed countries of the European Union, the Western Balkan countries including Kosovo, stagnate considering implementation of eHealth strategies (Piha, 2015). The slow pace of eHealth development is due to the lack of financial funds and political support. However, the results also show there are also promising strategic projects within the 
Western Balkan countries, especially in Serbia and North Macedonia. As mentioned above through the National Health Strategy, (now) North Macedonia in 2011 started implementing (MyTermin) in order to improve clinical appointments and to reduce the long waiting times of doctors' checks and the diagnostic tests. Initially, (MyTermin) was used in three public institutions, but quickly expanded to public hospitals and primary care providers, but further development continues. All public and private health institution in North Macedonia have already included in the (MyTermin) system into all healthcare services in these institutions, referrals and prescriptions, electronic health cards for citizens and electronic health records for each patient (Toçi, 2015).

The cloud-based system is a requirement of the North Macedonian National Health Strategy and designed to be scalable using modular programs, solutions that can be integrated with each other and with other healthcare applications. This system is combined with the main state system in eHealth services. In this system, the modules include useful services such as registration for organ transplantation, and joint decision-making on health (Arak \& Wójcik, 2017).

(Velinov et al., 2015) mention the Ministry of Health in the course of execution of National Health Strategy of North Macedonia, through the Health Insurance Fund, plans to integrate automated processes and practices at all levels of healthcare, including pharmacies and the administration. In the reports of the Ministry of Health of North Macedonia, the progress is documented, the project (MyTermin system) significantly reduces the waiting times for diagnostic images in health institutions because of the identification of false patients, the indetermination of the work of the doctors with minimal schedules as well as the unused time within the working hours (Atanasovski, Bogdanović, Velinov, Stoimenov, \& Sahpaski, 2017).

(Atanasovski et al., 2017) quoting a 2015 survey by the Ministry of Health, claim over $80 \%$ of Macedonian patients reported being satisfied with the system, doctors noted improvements in the patient notification if their appointments were cancelled or delayed. According to the results of this survey over $70 \%$ of patients were satisfied with the appointment and reduced waiting times at the doctor's office and fewer than $7 \%$ of patients failed to meet their 
scheduled meetings at their doctors). Based on the National Health Strategy Report of North Macedonia, the Ministry of Health had gradual access to building a more complex information system. In fact, this is a huge investment and takes time for multiple tests, for the Ministry of Health, the full implementation of this system remains a challenge. Especially budget constraints for the implementation are critical, the Ministry capable to afford the price of such a large and complex software (Atanasovski et al., 2017).

The implementation of the health information system in Serbia is far more developed compared to other Western Balkan (Vučetić, Uzelac, \& Gligorić, 2011) summarizing the National Strategy of Health of Serbia, conclude the implementation of the health information system has produced satisfactory results. As a result of drafting a lucid and straightforward national health strategy, Serbia has benefited from several European Union projects closely related to the WHO's Strategies for Developing Countries. First Serbia's initial program was the development of the SIS system for Basic Health Services and Pharmaceutical Services, that same national project was extended from an initial project to a major project, which was initially tested in 4 hospitals and focused on standardizing and expanding technological infrastructure. In the framework of this project, the Ministry of Health was obliged to change the Law on Health, which paved the way for the use of information technology to improve health services in the country. Among the main goals of the Ministry of Health to improve, the health service was to offer a lower cost service and eliminate paper service through the digitalization of the processes. Based on the objectives of the National Health Strategy of Serbia, the Ministry of Health continues to expand with additional objectives, which include the development of new modules in the systems, such as health reports, technical reports, reports on the electronic healthcare portal (Milenković et al., 2012). These objectives are also guidelines for the development of an analytical system that provides real-time information on patient diagnoses. Therefore, based on the above data, Serbia compared to other Western Balkan countries is on the right track to implement recommendations from the WHO Strategies and Reports (Atanasovski et al., 2017), (Vučetić et al., 2011). 


\section{$2.4 \quad$ Kosovo Health Strategy}

The objectives of the National Health Strategy of Kosovo are almost the same as other Western Balkan countries because Kosovo and most Western Balkan countries have similar history heavily influenced by the political conflicts of the last three decades. Whereas, regarding the implementation of the National Health Strategy, Kosovo has stagnation. Due to the fact that the Ministry of Health in Kosovo is the institution responsible for the management of the health sector in the country, in 2011 has carried out a Strategy on the Health Information System in Kosovo 2010 - 2020, which aims to inform the citizens about diseases and treatment procedures. This strategy provides the patients with the opportunity of being informed about the diagnosis, specifics of their treatment etc. whereas the professionals of the health sector have the possibility to search for proper guides, to improve their knowledge and to have an easy approach regarding the files of patients. (MoH, 2010c).

Obviously, the strategy which is concerned with the healthcare system attempts to analyze and provide in-depth scrutiny of the statistics of the existing system and to carry out a multifaceted strategy to develop the necessary infrastructure before 2020. The most important pillar of this strategy is the initiation of evidenced-based decision-making processes at all levels. This is the only way of achieving fruitful outcomes in the Health Sector Strategy $(\mathrm{MoH}, 2010 \mathrm{~b})$. The purpose of this document is to identify, through addressing the healthcare system and analyzing the existing Health Statistics System, the best ways to enable Kosovo to create the necessary infrastructure for health intelligence development and routine application before 2020, evidence-based decision-making at all levels, and to achieve the goals of the Sector Health Strategy.

However, the Health Information System has not yet been implemented, but as far as the institutions are concerned, they have anticipated being the broadest reform in the health sector $(\mathrm{MoH}, 2010 \mathrm{c})$.

According to the Kosovo Health Sector Strategy 2017-2021 (MoH, 2010a), the reform of the health system is based on maintaining and improving the health status of the population, ensuring sustainable financing and reorganizing the health sector $(\mathrm{MoH}, 2010 \mathrm{c})$. All this is planned to be done through the full functionalizing of the health information system, the clear 
separation between purchasers and health service providers, the strengthening of the financial sector's financial base, the promotion of quality and integrated health services starting with the services of mother and child, promoting and implementing the concept of family medicine and mental health services in the community, increasing the supply of medicines, setting up services for cardiovascular and oncological diseases, increasing the managerial autonomy of health institutions, functionalizing the system monitoring and evaluation. The strong focus is given to oversee the implementation of the project. Important vehicle implementing the national health strategy in Kosovo is to establish, maintain and develop a Health Information System (HIS), which provides electronic access to information to support decision-making on healthcare institutions, resource management, patient management, disease management, population health management, pharmaceutical management, participation in international organizations and meeting EU requirements for social cohesion, public health, and participation in the open market. Among the main objectives of this strategy are: to create and develop HIS infrastructure including legal infrastructure, organizational infrastructure, human resources bases and facilitating economic resources; to establish and further develop electronic processes and methodologies for collection, storage and analysis of data, setting up electronic information technology infrastructure; further development of the evidence-based decision-making method at all levels of the healthcare sector and for all participants in the healthcare system; as well as the full integration of the private sector, the creation of electronic patient file, as well as preparation for the establishment of hospital information systems.

The plan for the implementation of the National Health Strategy in Kosovo is foreseen to be divided into three phases. The first phase involves the necessary actions for infrastructure design and implementation of HIS pilot systems in key regions. The second phase concerns the planning of scaling out HIS system piloting in routine practice. After evaluation of the pilot, application of modules and processes for quality monitoring and cost-effectiveness of health services, and also with automation regular reporting of a significant number of health indicators will be enhanced. Whereas the third phase addresses infrastructure development, technological operations supported the full-scale HIS operation across Kosovo and expanded 
additional functions in proportion to the objectives of the National Strategy and the WHO Strategy (MoH, 2010c), (National Audit, 2017a).

\subsection{Summary}

All health system strategies in European countries should follow WHO recommendations, both in the level of locally and internationally. Based on the above research, WHO recommendations and strategies are especially needed for developing countries, such as the Balkan countries. The WHO's main goal under the 2020 Strategy is that in European countries, the national or local health management will be replaced by standardized and unified health systems so that they can be interconnected. The WHO Survey for European Countries is primarily has 8 key points: eHealth foundations, Electronic Health Records, telehealth, mHealth, eLearning in health, social media in health, health analytics and Big Data in health, as well as Legal frameworks for eHealth. Based on WHO survey results, recommendations are for states to use the WHO's National Health Strategy and integrated the International Telecommunication as a basis for developing their national vision as well as tracking the action plan and monitoring and evaluation frameworks for eHealth. Compared to the developed countries of the European Union, the Western Balkan countries including Kosovo stagnate considering implementation of eHealth strategies. The slow pace of eHealth development is due to the lack of financial funds and political support. However, the results also show there are also promising strategic projects within the Western Balkan countries, especially in Serbia and North Macedonia. The implementation of the health information system in Serbia is far more developed compared to other Western Balkan. The objectives of the National Health Strategy of Kosovo are almost the same as other Western Balkan countries. Whereas, regarding the implementation of the National Health Strategy, Kosovo has stagnation. As shown by the research, the successful implementation of a Health System Strategy in Kosovo requires a WHO roadmap, as well as the adoption of best practices by neighboring countries, as seen in the Balkan country during the implementation of the their strategies have almost the same challenges as Kosovo's. 


\section{Organization of Healthcare}

The healthcare system in Western Balkan countries, especially in Kosovo, is significantly based on the standard of WHO model, which is characterized by central planning and service specialization, where hospitals are classified in four ways:

- $\quad$ Type of services provided,

- $\quad$ Number of beds,

- Ownership,

- $\quad$ Length of stay (acute or chronicle patients).

In Kosovo this system is regulated by the Ministry of Health through the Law on Health No. 2004/4, which distinguishes three main levels: primary, secondary and tertiary (MoH, 2010c). The respective municipality funds the primary level and the spectrum of its services is more general, this is the first institution to be contacted by the patient. The secondary level provides more specialized services and is funded directly by the Ministry of Health. As well as the tertiary level, in addition to providing specialized services, it is responsible for higher education and realization of academic and scientific research projects. Services in each of the public institutions should be partially paid by the patient (co-payment) and partly through subsidies or funds from the Ministry of Health. Legally the division of activities among healthcare levels is carried out in accordance with the national health strategy and based on the principle of primary health care as the starting point. Access to healthcare is provided 7/24 without interruption, in forms of regular work, shifts, guardianship, and work within the emergency service, in accordance with the schedule set by the health institution. The completeness and continuity of health care services at the three levels of the organization is ensured by the full implementation of the referral system from the primary to the secondary level, from secondary to tertiary level (Hodzic, 2017).

\subsection{General Practitioner (Family Doctor)}

(Hodzic, 2017) summarizes in the Western Balkan countries, especially in Serbia, since 2012, primary care regulation has been developed, legislation reforms for primary care are related to the assessment of primary care service delivery and organization, including sharing of 
primary and secondary care provision, the introduction of "General Practitioner" system and the continued decentralization of primary care in favor of municipalities. For example, General Practitioners have a very limited role in emergency care and rarely do small-scale surgery or other medical interventions for their patients (Stubbs, 2009), (Milenković, Milenković, Vujin, Aleksić, \& Radojičić, 2012).

\subsection{Outpatient Care}

Among the key objectives within the Western Balkans health strategies is to provide ambulatory treatment for patients, especially in specialized clinics, to improve clinical effectivity, reduce the duration of the average stay, reduce the number of hospitalization and other reductions to improve patient acceptance, healthcare management and strengthen coordination between service providers, particularly when it comes to outbound patients in outpatient care (Peterson, Hamilton, \& Hasvold, 2016).

\subsection{Tertiary Level Health Institutions}

In most Western Balkan countries, the progressive health system provides services. Institutions at the tertiary level of health care are general and special hospitals with defined constituent units, Also as part of the tertiary health care institutions are the university clinical centers, university clinical dentistry center, National Public Health Institute, the National Center For Occupational Medicine (Chipman, 2016).

\subsection{Homecare - Family Care}

In most Balkan countries, home care (family care) is still not solved properly. (Chipman, 2016) argue the given rate of the ageing population in Western Balkan countries, especially in Serbia, home care should be provided and made available to patients on a much wider extent. National health strategies of the Balkan countries claim homecare needs to be integrated with primary care services, social services and long-term care. While WHO suggests increasing community care and shortening hospitalization, home care services can also be an effective response to problems stem from ageing populations. 


\subsection{Rehabilitation}

In developing countries, such as Western Balkan countries, the tasks of rehabilitation mainly fall on secondary care, which includes a complex set of services provided to community hospitals and general practitioners' offices after an assessment by a primary care practitioner. Secondary services include treatments and reconstructive procedures (Sopjani, 2016).

\subsection{Prevention}

The comprehensive concept behind health services providing curative care is not valid unless preventive measures are not taken into consideration. As matter of fact, the role of health institutions in the Western Balkan countries, according to their national health strategies, is no longer only in providing curative care, but also an active and important role in providing preventive and promotional health care. Health institutions need to have a critical assessment of preventive activities, including health promotion clinics, in order to prevent illnesses. Preventive and promotional efforts should be so effective that a child should have good health with a minimal need for curative consultations and less need for hospital admissions and hospitalization (Arak \& Wójcik, 2017).

\subsubsection{Awareness}

The need to improve healthcare and to support a better way of life, the role of nurses has become more significant. As a result, not only they are caring for the patient and doing other medical tasks, but they are also playing an essential role in raising awareness about healthy living, saving good conditions. Any interaction between the patient and the nurse is an opportunity for exchanging knowledge about health issues. While doctors are focused on diagnosis and treatment, nurses are educating patients, consulting their problems on how to prevent illnesses, in general how to improve their health. When people take more care of themselves then the chance of having a disease is lower, so they put less weight on the healthcare system. Community awareness is an important aspect of preventative healthcare, as well (Mountford \& Kessie, 2016), (De Lyon, Neville, \& Armour, 2017) . 


\subsubsection{Fitness}

Regular exercises are a major factor for a healthy lifestyle. Numerous researches (DeLyon, Neville, \& Armour, 2017) show the benefits of performing regular exercises, not only that they help in losing weight and stop the development of various diseases, help maintain mental health. Low fitness activity can increase the risk of developing different diseases but regular exercises help to:

- Improve muscular and cardio-breathing skills,

- Improves bone health,

- $\quad$ Reduce the chance of having hypertension, heart disease, diabetes, cancer,

- $\quad$ Reduce depression and other mental problems.

Kinesiologists have always been interested in physical activities, fitness, and their relation to health problems. There is a consensus now showing that engagement in physical activities and exercises throughout life helps positively increase the number of healthy years and wellbeing. WHO currently recommends that adults should have an intensive activity of 150 minutes a week, children should have an activity 60 minutes a week (Ziefle, 2011). Supporting fitness as a key point of public health has come from various stakeholders such as public health researchers, politicians, industrial organizations, politicians (DeLyon et al., 2017), (Kim, Wang, \& Mahmud, 2016).

\subsubsection{Sport}

Technology revolutionizes sports in several respects. Many new creations are used to improve the performance of an athlete, while others affect the justice of the game. Improvement in health security, monitoring solutions are significant, electronic wardrobe or electronic clock allows real-time tracking of an athlete's health. Policy and technologyrelated liaison with doctor's results in improving mental health conditions, and dehydration incidents, heart attacks, and many other worse incidents have fallen significantly. In the developing countries we still do not have a splendid product worth mentioning in the field of sports, but simple applications developed by IT professionals have found application for a 
part of the society that is trying to advance health and technology (DeLyon et al., 2017), (Arak \& Wójcik, 2017).

\subsubsection{Nutrition}

Nutrition plays a crucial role patient healing, and most of the hospitals are working in this direction for patients to have more information about nutrition to improve their health. Numerous studies have shown that good nutrition has resulted in improved health after hospitalization. Food has an impact on the treatment of obese people, critically ill patients, people who have allergies to foods, and helps in many different types of diseases. At present, nutrition awareness in the Balkan is being applied exclusively by fitness centers but there is still no plan by the health minister to become vigilant through state health policies (DeLyon et al., 2017).

\subsubsection{Lifestyle coaching}

Recent researches conclude that daycare is costing more and more, the human resource team is looking for different opportunities to lower the cost of healthcare and maintain the healthy condition of people. "Health coaching" is an effective method to improve health at a relatively low cost. Health coaching can positively influence the fight against chronic diseases such as diabetes and high blood pressure. Health coaches are trained to work with only individuals so they can always work according to the conditions that fit the patient in question. Health coaching helps many people who work hard and live a stressful life, and whom the official healthcare has left behind. Above all people do not change for one night but they have to work continuously to achieve their goals for a healthy life by creating a daily, monthly regular check-up even though it needs extra effort from most of them (Albahri, 2018), (WHO/EU, 2016).

\subsection{Stakeholders}

All potential customers and contributors to the healthcare system in the countries of Europe and the Western Balkans, including Kosovo, are seen as important actors in improving health services. Understanding the nature of the health system they are grouped in many areas, such as social workers, policy-makers, service providers, consumers, health workers, etc., who 
with better coordination can contribute to better functioning of health services (Hammond, 2010), (Peterson et al., 2016). The strong interconnections are illustrated in Figure 1.

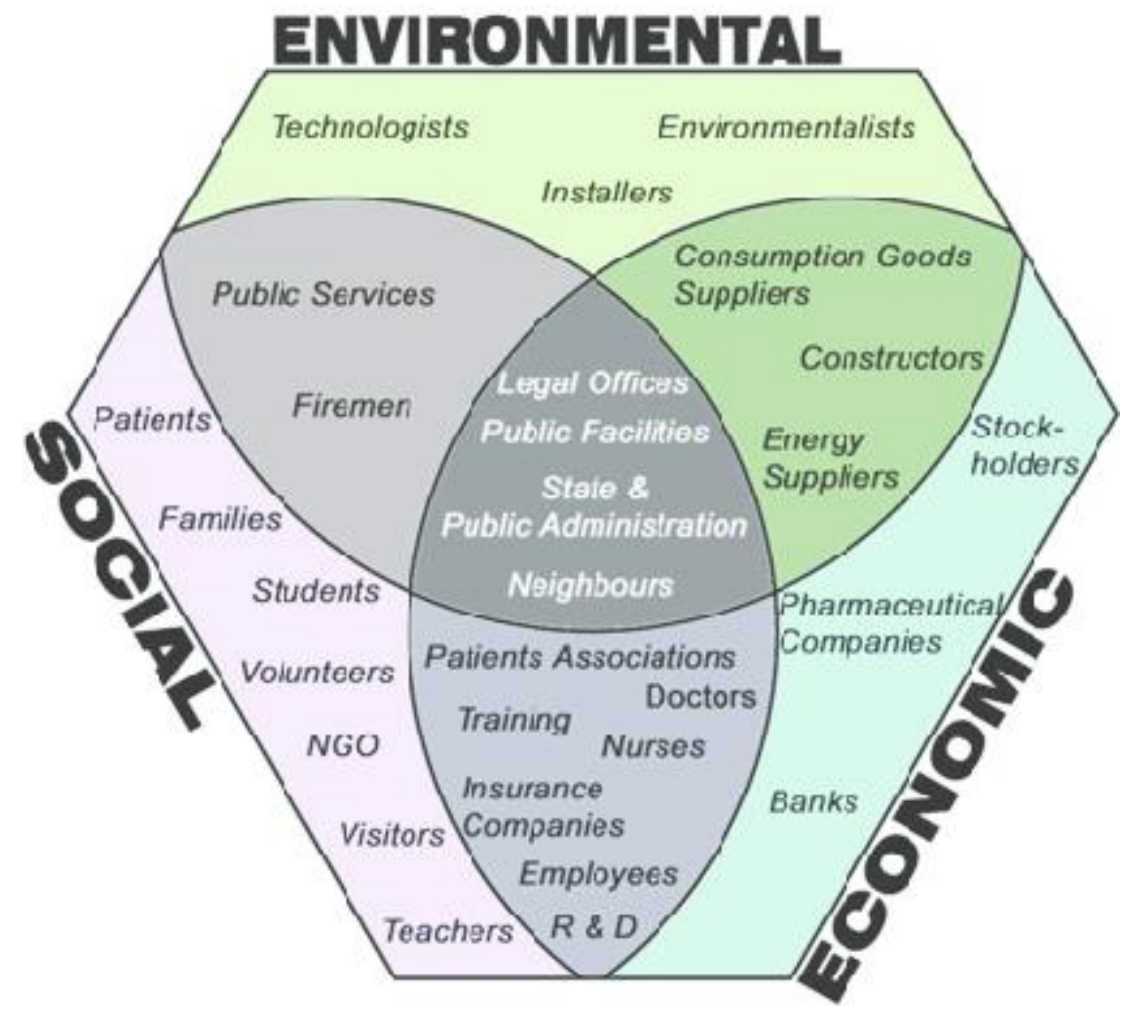

Figure 1. Stakeholder structure (Capolongo, 2015)

\subsubsection{Social policy-makers}

Public health policies in Western Balkan countries are under the control of the government, respectively the Ministry of Health, which compiles healthcare policies and implements the laws of a non-discriminatory and responsible system for healthcare based on professional analysis and scientific data. (Hodzic, 2017) give an outline of healthcare legislation. The Ministry of Health sets standards and guidelines for the health sector while respecting the relevant international standards. The Ministry of Health as a policy-making body also undertakes all the measures prescribed by law to carry out the right actions with the purpose of implementing and protecting the rights of citizens, residents and other actors of health services in healthcare. In the last three decades most of the Balkan states have been faced with wars, and for the people of these countries, as the case is with Kosovo, has brought not 
only financial and infrastructure problems but also illnesses and bad environment not conducive to the health of the population in place (Hodzic, 2017).

According to the official statistics (KAS, 2013) the average age of the population in Kosovo is 30.2 years, policy-makers see it as necessary to focus on the planning of interconnection between human resources and technology in order to optimize the cost of services and time. Because of this interconnection improvement of morbidity and mortality rate of the population is one of the key problems for the population in Kosovo due to the lack of proper health services. In Serbia and North Macedonia, the population is ageing, the average age around 45 years in comparison with Kosovo, where the average age is younger. In this case, policy-makers in the mentioned two countries are targeting the health system to reduce costs and reduce hospitalization time. Therefore, monitoring the health of patients through the National Health System since elderly patients get continual treatment, they see it as an effective solution.(Arak \& Wójcik, 2017), (MoH, 2010c).

Whereas, in recent years, Bosnia and Herzegovina has adopted many strategic policies that seek to improve the health service, including: "The latest Strategy for Improving the Rights and Status of People with Disabilities in the Federation 2016-2021"; "Strategy for rare diseases 2014 - 2010"; "Resolution on Diabetes and the Strategy against Diabetes 2014 2024"; "Policy and Strategy for the Protection and Improvement of Mental Health in the Federation 2012 - 2020” (Arak \& Wójcik, 2017).

\subsubsection{Senior and junior medical staff}

One of the interest groups is senior medical staff, including doctors, management staff, and healthcare specialists. The senior medical staff has an active role in the care and health of the patient because it is responsible for implementing the principles and procedures of the medicine for the prevention, diagnosis, and treatment of patients. Therefore, it is necessary for the senior medical staff to have the patients' confidence in the treatment of their health problems, but also in the prevention of other illnesses that may arise, in order to create the possibility and the space for the patients' professional treatment ( WHO, 2006).

All healthcare professionals require a variety of generic attributes that deserve the trust of patients who support the therapeutic relationship. These qualities include good 
communication skills, ability to work as part of a team, non-judgmental behavior, sensitivity, and integrity. However, in addition to possessing these shared attributes, senior medical staff should be able to assess healthcare needs of patients taking into account their personal and social circumstances by applying their knowledge and skills to synthesize information from different sources in order to achieve accurate diagnosis and identification of the steps to be taken to treat the patient.

In all Western Balkan countries, the health sector is facing the problem of fleeing doctors, and also nurses from the country, looking for the same work in the European Union because of the better living and working conditions. According to data published by (Christodoulou, Dunbar, Gáspár, Jaksa, \& Krapez, 2008) also by only in the last decade over 1700 doctors from Serbia, North Macedonia, Albania, Serbia and Kosovo have fled to work abroad, which makes this problem recognized and centered on policy development regarding further health and education in the region. This problem is even greater for Kosovo, given the small number of medical staff so far compared to the population. Based on (KAS, 2013) statistics, the population in Kosovo for 2017 was estimated to be 1,798,506 residents, while the medical and nursing staff at the secondary and tertiary healthcare level was 1441 doctors and 3966 nurses. Compared to the countries of the region and the European Union, the low number of doctors and nurses gave the problem of lack of health staff in Kosovo and in other Western Balkan countries, eHealth is a good opportunity for the proper functioning and providing health services to patients. eHealth might counter-balance the lack of health staff, especially in the technical procedures, eliminating the time lost in these procedures, and direct the focus of health staff to improve diagnoses and other health services (Chipman, 2016), (DeLyon et al., 2017).

\subsubsection{Nurses}

The role of nurses in the health service in most of the Western Balkan countries has so far been more administrative and technical, providing technical assistance to doctors rather than providing healthcare services to patients. However, with the public policies of these countries, the role of nurses is being promoted to change in the direction of the professional role, with the aim of providing professional health services to patients. In Kosovo is already 
a requirement for nurses to have an undergraduate nursing diploma, where not long ago the nurses have only secondary education. The main focus on advancing the role of nurses at the professional level is to have a decision-making role in providing health services to patients, especially in preventative measures for patient health care (DeLyon et al., 2017), (Sopjani, 2016).

\subsubsection{Patients}

One of the key components for efficient healthcare service is an effective relationship and communication between health workers and patients. (National Audit, 2017a) investigated public policies in all Western Balkan countries and concluded patients are entitled to treatment with respect and sincerity and, whenever possible, stimulate their involvement in decision-making on their care health. For patients' participation, sincere communication between the treatment team and the patient is needed that information and knowledge can be shared, giving the patient a sense of control and responsibility, including patients suffering from mental problems, to benefit and accelerate rehabilitation from the active involvement. As one of the key contributors, patients can make an important contribution to the surveys conducted by health and professional institutions related to healthcare, thus identifying the problems and challenges of treating them in healthcare institutions. The information gained from patient surveys can be used by health professional staff to improve health services because this is a good indication that the patient is a real stakeholder of the healthcare system.

\subsubsection{Family}

Due to financial problems and poor health services, the family has a very important role in patient care, especially when patients need long and complex rehabilitation. Therefore, families have a strong effect on the well-being of patients, a family approach to healthcare can help curb rising healthcare costs by both the patient and the healthcare providers. All Western Balkan countries through public policies should strengthen the role of families in the healthcare of patients because families can help promote health, reduce the risk of disease, and prevent disease initiation. Families also affect healthcare and treatment decisions (Hodzic, 2017). 


\subsubsection{Civil organization}

Civil society has the power to influence individual patient behavior by providing counselling and basic services for their own health situation or counselling for disease prevention. Civil society also has an active role in institutional behavior that drafts and implements policies for the health system, as well as the institutional behavior of health service providers through monitoring and delivery of services aimed at improving health policies and services in a place (Stubbs, 2009).

(Carrasqueiro, 2017) summarized why civil society plays an important role in promoting health and advancing the health agenda. Civil society (WHO, 2006) is a key factor in formulating, promoting and delivering change. In various countries including the Western Balkans, WHO has been at the forefront of forming innovative partnerships with civil society, including communities of the major population groups at higher risk (such as HIV-infected people) and non-governmental organizations advocating and provide services (WHO, 2011) In Kosovo according to (Gjorgjev et al., 2006) there is a relatively large number of civil organizations providing services to Kosovo citizens, the main areas covered by them with the provision of services are mainly social, educational, health services. Cooperation between a civil society with patients and health service providers is more than necessary, as both institutions and patients can benefit from the services provided by different civil society organizations, either by providing services or training to promote public health and disease prevention. Civil society not only influences counselling on disease prevention but can also affect the process of policy-making in healthcare. The impact of civil society on public policy is an essential necessity for improving policies and the health system itself and can contribute either during the public health policy process or during monitoring of the implementation. For example, by involving different civil society actors in co-operation with public institutions and international partners such as WHO, they can support the implementation of national health strategies in Western Balkan countries by monitoring the implementation process of the strategies or by suggesting improvement of these strategies, even occasionally delaying their implementation (Stubbs, 2009). 


\subsubsection{Church / caritative services}

(Gjorgjev et al., 2006) and (Arak \& Wójcik, 2017) explore in their report social protection and social inclusion in Western Balkan countries, namely: Albania, Bosnia and Herzegovina, Kosovo (under United Nations Security Council Resolution 1244), Montenegro and Serbia, based on a series of reports completed by independent churches in August of 2008. In accordance with its mission, the church's healthcare activities (both Catholic and Orthodox) tend to differentiate themselves by serving and advocating for people whose social condition puts them at the margins of the society and makes them particularly vulnerable to discrimination: the poor, uninsured people, children and the newly born; single parent, elderly, those with incurable diseases, racial minorities, immigrants and refugees. Especially persons with mental or physical disabilities and also Roma minorities as the most discriminated group in the Balkan state (European Commission, 2018).

Palliative care is provided by a special team of health professionals, including doctors, nurses and other specialists working together, providing services and advice to improve not only the patient's quality of life but also give help to their family. This form of health service is appropriate at any age and at any stage of a serious illness and can be provided along with curative treatment. Palliative care is a specialized healthcare service, which is offered to patients with serious illness, e.g. such as the case of severe diseases of cancer, Alzheimer's, Parkinson's, etc. The palliative care professionals focus their attention on providing services and consultations to the patient and family in relieving the symptoms and stress caused by a serious illness both for the patient and families who are giving care to the patient. Therefore, coordinating the activities of professionals, doctors and the family and the patient is an important factor in the complexity of healthcare and it is far from a quasi-optimal solution (Gjorgjev et al., 2006), (Stubbs, 2009).

\subsubsection{Voluntary services}

One of the most important civil society groups is the Voluntary Service group, which in the health service makes a major contribution to the public sector with assistance saving money and providing better services, especially in the prevention. Volunteer organizations or volunteer groups, whether local or international, provide free voluntary counselling, services 
to citizens, in particular, public health prevention, promotion services, and advice, especially in cases when the high cost for receiving services or advice from public or private healthcare institutions is a serious barrier. In Western Balkan countries, volunteer service groups also play an important role in public awareness campaigns and promotion of public health in the country, organizing information days and other information campaigns, public activities for making more sensible the public for dangers and negative consequences. In Kosovo on the International Day Against Breast Cancer, public training is organized in the sense of sensibilization of women for breast cancer measures, with a view to preventing breast cancer, either through routine or breastfeeding check in the mobile mammogram, such arrangements are provided as a free service or blood donor bodies in universities for the benefit of people in need (Carrasqueiro, 2017), (Hodzic, 2017).

\subsubsection{Health industry players}

\subsubsection{Pharmaceutical companies}

The pharmaceutical industry plays an important role in the cooperation between healthcare institutions and is a driver of a network of customers. Interstie parties are also comprised of doctors, caring institutions and patients as consumers. Moreover, part of the interested parties are pharmacists, health insurance funds, healthcare politicians, and pharmaceutical wholesalers (Imasheva \& Seiter, 2008). Physicians, because of their strong influence on decisions related to the patients' retention should be considered as interstate parties in a broader sense. An already identified problem shows that pharmaceuticals' representatives of the industry are developing a connection with doctors, rather than patients. Doctors describe the medication of a certain company; it may increase sales by communicating with pharmaceutical companies as well as suggesting replacing some other, similar product. There are three main stakeholders in the pharmaceutical industry, which include producers, patients, doctors. Based on the current situation, it is foreseeable in the Balkan region the focus will be given to reimbursement that is still in the hand of private insurance companies. At a later stage, it can be expanded to cover some medical equipment and other standardized technologies and thus a good model of cooperation between the health system and pharmaceutical industry (Danko \& Petrova, 2014). 


\subsubsection{Healthcare vendors}

Vendors have a significant role and are of great interest. Amongst many other vendors, IT vendors are of our interests. They are the private seller of software systems and technology for managing and developing database applications that can help integrate healthcare information systems. Their products also help third parties such as pharmacies, insurance companies through applications; databases help these companies to be integrated into the state health system. A good example is the creation of data integration modules for both the pharmacies and the insurance companies collaborating with healthcare institutions. In the absence of laws, often vendors are in conflict with state-owned public data storage and data protection policies, where they must protect the confidentiality and privacy of data that they can expose during communication through their systems (Langenhove, Decreus, Rogala, Olyslaegers, \& Whitehouse, 2013).

\subsection{Smart gadget manufacturers}

Healthcare has undergone drastic changes with the advancement of technological developments, ranging from anesthetics and antibiotics to resonance imaging magnetic scanners and radiotherapy. Companies that develop technology equipment have helped physicians to better diagnose and treat patients' illness. Recently, we have seen more users of healthcare monitoring devices, thanks to the advancement of technology, low prices of electronic devices such as a smartwatch, sensors, mobile devices, analytic software, etc. Some of these devices include monitoring of diseases such as breast cancer that shows metabolic disorder, as well as the baby's stomach in which case measuring the amount of oxygen that the baby is sucking. The good thing about these devices is that the data is stored in the system and is easily accessible by doctors who have the best update which enables them to improve the expected results. There are several challenges the technological equipment producing company are facing amongst others the standardization is under different legislations in the producers' countries than in the customers' country, another challenge remains the performance of the switchover in different environments affecting the decisions of physicians who rely on technologic equipment as a source of information for decision making (Pashazadeh \& Navimipour, 2018), (Yaseen et al., 2018). 


\subsection{Summary}

Understanding the work and health organization enables us to understand the requirements / needs, as well as the role of users in the digitalization of the health system. The healthcare system in Western Balkan countries, especially in Kosovo, is significantly based on the standard of WHO model, which is characterized by central planning and service specialization. The organization of the health system in most Balkan countries is at three levels: primary, secondary and tertiary. Communication between the three levels and their interconnection with all stakeholders, medical and non-medical staff, turns out to be a need and one of the key points for the proper functioning of the health system. Each stakeholder plays an important role in improving health services. Based on the behaviors and work roles of each of the stakeholders, which have been elaborated above, there is a need to digitize their work processes. Therefore, any proposal for a healthcare information system should have as its primary objective the interconnection of stakeholders, using different technologies, based on the needs of the stakeholders, and have interoperability between them. 


\section{State of the Art - eHealth in the Literature}

\subsection{ICT Enter to Medical Sciences}

The review reflects to the literature that seeks to understand the health systems and the impact of Information and Communication Technology (ICT) on the healthcare sector. Especially case studies of different countries are of our interest as a basis for exploring, analyzing innovation, development, benefits, advantages, strengths, weaknesses and disadvantages of these systems. By understanding and analyzing these factors, we are able to contribute to the medical science field and ICT opportunities in health care, knowing that health care sectors face costly and complex services that have a major impact on the economy and the everyday lives of peoples (WHO, 2012).

Nowadays, the widespread use of ICT has permeated through almost every aspect of life, including the healthcare sector. (Hassanalieragh et al., 2015) highlight ICT can contribute to the dissemination and management of health information related to patients' diagnosis and treatment. ICT also has the potential for helping patients to solve (partially) their own problems. The health information system is the establishment of a standardized interchange platform of patients' data, through the network infrastructure, hardware equipment's and software solutions. eHealth is an emerging field of medical informatics (Krishnamurthy, 2012).

In a broader sense, the term using information and communication technology characterizes not only a technical development, but also a new way of working, a different attitude, and strong commitment for networked, global thinking, improving healthcare locally, regionally, and nationwide.

According to the study of (CAST, 2013) electronic data interchange is the most dominant form of communication and nowadays as it replaces the traditional statistical reports, analysis, and monitoring, it increases the opportunities for interaction between patients and medical services. The electronic data interchange moreover favors the data validity, providing data on real time, and these features enhance the quality of decision making in the clinical, administrative and management areas. 
Similar to other industries, the healthcare sector's nature has changed over time from a relatively stable to a dynamic industry. According to Patrice Cristofori (Hammond, 2010) member of the Board of Directors of the European Association of Health Telematics (EHTEL), more and more patients tend to have access to information and manage their personal data through mobile devices. People in many developing countries use advanced technology due to their enhanced communication practice. The result of the intensive use of advanced communication technology opens a pathway to implementing electronic health monitoring system assisting many patients.

In addition, the health information system has evolved through different technological stages. (Greenspun \& Coughlin, 2012) recall mobile health (mHealth) has emerged as a special form of eHealth. This term refers to the delivery of healthcare services via mobile communication devices (such as smartphones, portable computers and tablets). mHealth is an interdisciplinary field with various disciplines such as healthcare professionals (HCPs), biomedical engineers, and information technology specialists working together to support applications used by patients. Other handheld hardware/sensors (such as a spirometer accessory) can be attached to a mobile communication device, and a health application subsequently displays and analyses the data. (Greenspun \& Coughlin, 2012), (Mshali, 2018).

\subsection{Digitalization in Healthcare}

Several authors mention (Mshali, 2018), (Bhavnani, Narula, \& Sengupta, 2016) currently digital solutions and electronic health records are not available in a wide aspect, they remain limited to general living habits and do not fully document the patient's illnesses. However, with the advancement of eHealth most patients will have an active role in diagnosis and treatment. A study prepared by the consultant firm Roland Berger outlines; the internet and widely available digital information have already brought a major change in the role of the patient. Patients now have access to health information, as for education purposes and to communicate with other patients via the internet about their health status and treatment potential (Roland Berger, 2016).With the advancement of technology, the traditional patientdoctor relationship will no longer be as a central role as it has been so far. Instead, we expect the online platform to be the main interface between the patient and the doctor, where through 
digitalization; the best medical devices connected will allow real-time monitoring of clinical parameters. Online platforms that provide lightweight tools to be used to compare and select a specific doctor or clinic will be available to patients. Therefore, doctors and hospitals need to review their value, proposing and creating their own individual business models to put them away from domestic and international competitors (Ziefle, 2011).

This is the only option that they have if they want to remain important players in a field where large data systems like IBM Watson Health are able to diagnose diseases only with the strength of digital patient information (Tresp et al., 2016). This sends up to a new kind of doctor-patient relationship, in which the patient becomes a partner to establish appropriate (or even proactive) treatments to improve the accuracy and timely intervention of doctors.

\subsection{Popularization of ICT in Medical Treatment}

\subsubsection{Digitalization in diagnosis and treatment}

Digital technology has the power to change the relationship between patients and their General Practitioner (GP) service points, in the same way as relationships in other areas of life have changed, such as the bank's finance management or different online shopping platforms (Taylor, 2015), (Tresp et al., 2016). To meet the demands of healthcare society, advances in information and communication, as well as medical engineering technologies, provide new or improved medical diagnosis, therapies, treatments, and rehabilitation opportunities. In those situations where patients cannot continue to be treated only in the institutional environment, individual and personalized care in the home environment of patients plays an increasingly important role. One of the most interesting aspects of eHealth is managing relationships between healthcare providers and patients (Cerdan, CatalanMatamoros, \& Berg, 2017). Fostering a good relationship with the patient will entice them to be loyal, to create mutual understanding, trust, and pleasure. In addition, a good relationship will encourage the patient to engage in decision-making during treatment. This will promote effective communication, which is often associated with improving physical health, managing chronic diseases, and better health related to the quality of life (Fairburn \& Patel, 2017). These services enable patients:

- $\quad$ Book or cancel appointments at the time that is appropriate for them 


\section{- $\quad$ Order recurring descriptions online (on regular basis)}

\section{- $\quad$ See some of their online information recorded by the GP}

Types of potential term bookings are managed by the GP at which the patient is registered, the repetition of the descriptions allows the patient to save time and money, as they do not need to visit the GP again in case of continued therapy as well the third possibility is to view personal health information from the mobile device such as allergies, descriptions, and previous therapies while not visiting their physician. Managing relationships with patients' needs to develop and grow constantly. In order for a good relationship to be dynamic, the organization that provides healthcare should be vigilant and aware of changing the needs of the patients during treatment but also after the treatment. On the other hand, when hospitals have an information system and are well managed, patients will always want to be part of it, because trust has already been built. In this case, patients know that if they face difficulties, the organization will resolve it professionally (Ziefle, 2011).

eHealth should be understood by the patient as systems that provide specific information to support the decision-making process at each level of an organization. Therefore, the main objective of health information systems is not to gain information, but to improve actions related to providing patient service and improving processes in hospitals. By using the eHealth Information System, medical human resources can be allocated as efficiently as possible, while at the same time treating patients is organized as well as optimizing waiting time (Albahri, 2018).

\subsubsection{ICT Enter into the treatment of patients}

Use of information and communication engineering technologies with medical machinery has made it possible for us, as healthcare researchers, to contribute to several areas in the digital medicine as tele-monitoring services, self-care services, notification services, emergency treatment services, and data archiving (Stachura \& Khasanshina, 2007). These services cover many areas in healthcare including monitoring of ECG, glucose level, stress, elderly people with chronicle diseases and patients' physical activity. According to Annette and Lynn (Ziefle, 2011) in terms of healthcare using ICT, a patient who needs permanent medical care will not be limited to be treated in specified areas, for example at home or in 
rehabilitation centers. The patient can move freely and live a relatively normal life, under the overall medical supervision. In regard to this perspective, the goal of providing healthcare services to any patient without constraint on time and location. The main beneficiaries of ICT in healthcare will be patients, while their relatives are the secondary ones, according to their medical treatment and the whereabouts they are treated or monitored: where they live (at home), the workplace or the environment in which they act. (Stachura \& Khasanshina, 2007) conclude that telehealth means the provision of health services at a distance using a range of technologies, such as telephone or video consultation to support the diagnosis. Telecare relates to the remote or enhanced delivery of care services to people in their own home or community. The concept of eHealth is the broadest concept, covering nearly all aspects of healthcare issues. It mainly involves two areas: infrastructure and services. Telehealth or telecare belong to the service part (Chaudhry et al., 2011),(Anker, Koehler, \& Abraham, 2011) draw the attention tele monitoring requires the use of technology and equipment and includes the collection of objective and subjective clinical data and the transmission of such data between a patient at a distant location and a healthcare provider through electronic information processing technologies. (WHO, 2012) report mentions eHealth is generally a matter of transferring, exchanging and managing data between public health institutions and citizens by using ICT solutions. The main target of bringing ICT solutions in healthcare is to seamlessly connect all stakeholders such as hospitals, insurance companies, general practitioners, the pharmaceutical industry, and regional public health agencies with patients at the core. (Piha, 2013), (Albahri, 2018) point out these advanced technologies for patient care enable remote patient monitoring, better transporting information to patients, improving access to health advice and quicker access to emergency services. For healthcare practitioners, the technologies also help to deliver better training and improve disease discovery, data collection, and management of patient records, thus enhancing service transparency and accountability. Functional health systems depend on harmonized and interoperable data elements between and within systems (Guedria et al., 2015). 


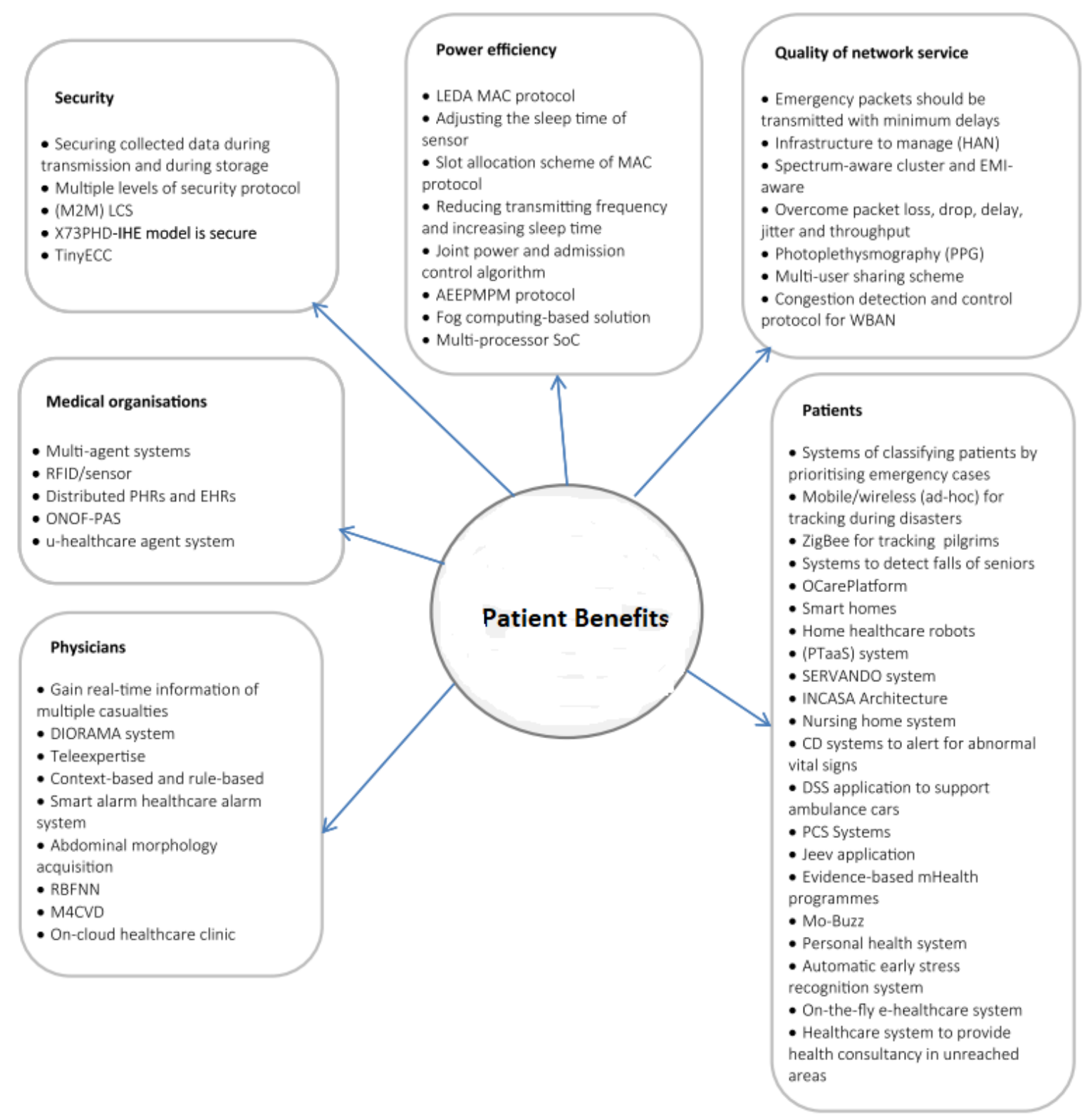

Figure 2. Patient Benefits through the ICT (Albahri, 2018)

(Stachura \& Khasanshina, 2007) in their paper concluded tele-monitoring services provide the opportunity of continuous and detailed information on a patient's status. Thus, it offers details of patient's position, context and environmental conditions (temperature, humidity), which are continuously monitored by special sensors located on the body or through devices such as web portals, mobile phones or tablets. (Rosa, 2011) shows in a study, monitoring systems like mHealth refer to smartphones, personal digital aids (PDAs) and pocket-based personal computer systems. This model allows patients to be fully mobile while undergoing 
constant health monitoring through utilizing various networks to communicate with the doctors and healthcare system as WiMAX, LTE or 5G (Lloret, Parra, Taha, \& Tomás, 2017). (Oleshchuk \& Fensli, 2011) mention another option for monitoring patient's health through ICT that is a smart health monitoring enabling continuous evaluation of the patient's health status. A set of significant biomedical parameters are constantly monitored through biosensors, which are located on or implanted in the patient's body. A wireless body sensor network collects and evaluates data from these Getaway devices. After evaluation, the results will show the current health status of the patient and stored in a database. This principle allows patients and healthcare providers to interact almost continuously and without interruption. Vital signs included are heart rate (pulse) (RR), blood pressure (BP), electrocardiography (ECG), oxygen saturation (SpO2), body temperature (temp) and respiratory rate (resp). (Chaudhry et al., 2011), (Yaseen et al., 2018), (Saleem, Derhab, AlMuhtadi, \& Shahzad, 2015) (Rosa, 2011) adds to the above-mentioned features, healthcare services are designed for enabling the provision of medical care to the patients. The traditional healthcare services are based on the doctor-centric paradigm. Essentially, they enable healthcare providers to assess a patient's health status based on information derived from medical examination and information stored in the patient's Electronic Health Records. Improving the quality of healthcare is an economic and moral necessity. (Jovanov \& Milenkovic, 2011) found patients can minimize the cost of hospitalization while being monitored in their homes effectively as in hospitals. System and real-time monitoring help identify emergency conditions for patients in an easy and fast way, and it is possible to solve the problem of the shortages of bed in hospitals by remote monitoring of some patients in their home. All the above benefits provided by the healthcare system focus on efficiency, which reduces workload over medical staff and early responses in the event of an emergency (Nadeem et al., 2015).

\subsection{Challenges of ICT in Medical Treatment}

In this section, I will give an overview based on the relevant literature, about the existing use of the technologies, the standards to be applied in the system, the wide variety of ICT applications in medicine. Based on a study prepared by (Ziefle, 2011) the purpose of this literature research is to highlight the challenges by using the supporting components that 
correspond to the technical and semantic interaction, as well as the whole system interaction process, another equally important objective to reduce cost and improve accuracy. This is a pressure on healthcare providers to start managing and sharing patient information in digital formation short, how to obtain information in order to improve the treatment. In more details, various challenges in telemedicine systems exist, such as medical action scheduling, communication between a patient and a hospital, knowledge management during monitoring, and enhancement of data security and risk management (Santos et al., 2016). Challenge about cost, various benefits of healthcare system technologies hardly can be accessed by developing countries because of their limited resources and infrastructure (Jovanov, Milenkovic, Otto, $\&$ De Groen, 2005). The daily living challenges elderly people are facing to pose problems for telemedicine systems. One of the major challenges is the need for assistance at home, a few people can provide hire or provide an independent caregiver for their elderly or disabled relatives (Ziefle, 2011). Numerous caregivers and caregiving organizations offer such assistance; patients with cognitive dysfunction, such as Alzheimer and chronic illnesses, these issues are rapidly becoming the main focus of improved healthcare delivery systems. Many developers and researchers have conducted studies on the various fields of telemedicine, by surveying these studies, we found that researchers are concerned about the challenges in telemedicine related to sensors in WBAN, smartphone applications, and servers in medical healthcare centers (Al Ameen, Liu, \& Kwak, 2012). The main problem areas, such as scalability, mobility, reliability, and energy efficiency in healthcare systems can be addressed by the recent development of ICT technologies. Cloud technologies, sensor technologies, IoT (knowledge of domain activity for modelling sensor coating networks) and big data analytics systems are emerging technologies and are still challenges and new technologies that engineers are being viewed as a feasible application (Shen et al., 2018).

The vision of any eHealth project reviewed in the literature aims to integrate information technology into a medical ecosystem. The correct selection and integration of communication technologies is still an open issue and needs to be addressed in the context of eHealth system information (Mshali, Lemlouma, \& Magoni, 2018).

The transformation of the healthcare system, which is needed to improve productivity and effectiveness, will rely on computer interaction to provide information when and where 
required, support decision-making, eliminate duplication of data, reduce delays, and avoid mistakes . Several authors concluded that the lack of common standards in data collection methods causes gaps in health data reporting as it may lead to important data that should not be reported (Krishnamurthy, 2012), (Pashazadeh \& Navimipour, 2018).

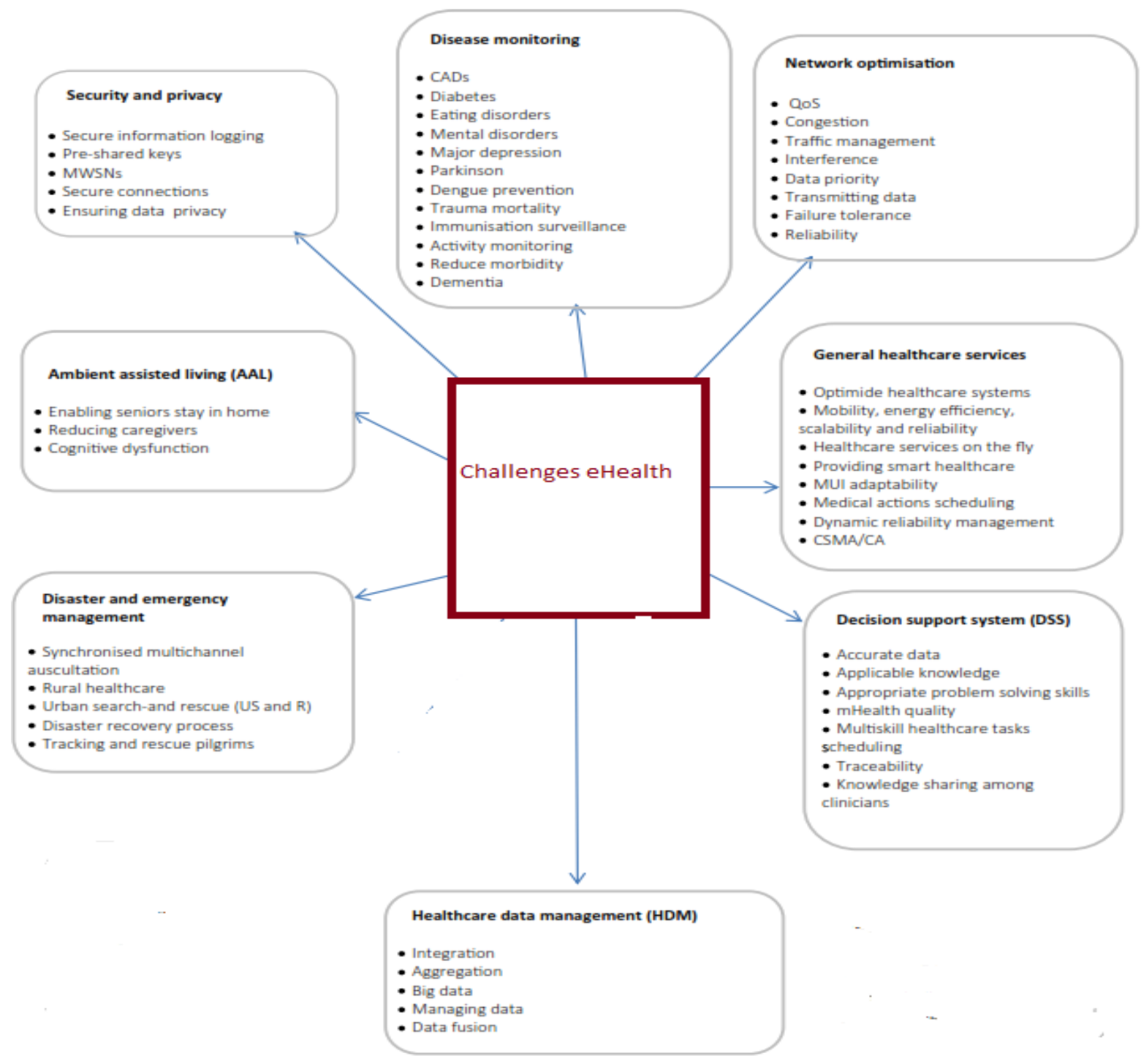

Figure 3. Challenges in eHealth System (Albahri, 2018).

The challenges for engineers and other stakeholders force integration of information technology into a medical ecosystem (Albahri, 2018). The authors conclude the ICT has a promising perspective due to the growing market of sensors and great opportunities in mobile devices that promote future changes in the healthcare system. Interaction between the 
existing system and infrastructure within the health system is key to providing medical services to patients, considering the economic conditions and the geographical situation.

\subsection{Cost Optimization}

Other experts (Maccallum \& Austin, 2000), (Kumar \& Lee, 2011) point out the value of health information is enhanced by making it readily accessible to decision-makers and by providing incentives for, or otherwise facilitating the use of information. To provide and carry out efficient and qualitative patient care, as well as accurate and detailed information about the status of direct, as well as indirect processes concerned with the health of the patient, it is worth having a serious and trustworthy database. Such information is closely related to medical examinations, to a wide range of departmental procedures, to laboratory outcomes, to medical checks, account management, pharmacies, etc. Better information contributes to better policy for decision making, health planning, management, monitoring and evolution of programs and services, including patient care, improving performance and overall performance of health services (Lee, Ghasemzadeh, \& Mortazavi, 2013). The basic assumptions that are implied in the information system are multidimensional: first, good quality data available in the shortest time and will be transformed into useful information that will influence decisions on a positive basis. Second, the results gained from data manipulation will lead to more effective and better, which will help to optimize cost and decision-making procedure. Undoubtedly, the process of information can be divided into the following components: data collection, data transmission, data processing, data analysis, tracking information along with planning and management of health services (Rolim et al., 2010).

Data mining is of significant importance when it comes to the healthcare system. A large amount of data which is available in the databases contains the utmost knowledge that needs to be elaborated (Pashazadeh \& Navimipour, 2018), (Woodbridge \& Tu, 2011). Indeed, it is extremely difficult, and almost impossible, for a human being to do so. As a matter of fact, we need an automated system, such as data mining, to find and process information (Krishnamurthy, 2013). 
When medical institutions apply data mining to their existing data, they can discover new, useful, and potentially life-saving, financial resources that otherwise would have left immovable errors in their databases. Implementation of this process is useful and costeffective in itself. The exploitation of data directly aids the organizations to acquire useful knowledge from their existing data at a minimal cost (Jovanov et al., 2005), (Kumar \& Lee, 2011). Data management aims to detect scams on credit cards and insurance systems. Similarly, these techniques can also be used to detect abnormal patterns in health insurance systems, and thus fraudulent claims may be discovered (Stachura \& Khasanshina, 2007).

ICT in healthcare, in particular, play an important role when patient and doctor are at a distance, and such systems are capable of reducing healthcare related costs and enhancing the quality of patient healthcare delivery.

Treatment of chronic diseases such as heart failure and diabetes is responsible for a disproportionate share of health care costs (Chen, Gonzalez, Vasilakos, Cao, \& Leung, 2011) - According to (CAST, 2013) analysis of the existing published telehealth literature, home monitoring for chronic diseases is one of the areas where telehealth has the greatest potential to reduce health care costs.

According to (Newman \& McMahon, 2011) improved access to healthcare at the most appropriate level, increased patient satisfaction, conducting in-home medication reconciliation and providing services resulted, early identification and treatment of disease exacerbation, reduced hospitalizations, reduced bed days, reduced emergency room visits, reduced health care costs, actively engage patients. If mobile phones can be tools for remote diagnostics, they also allow patients and healthcare providers to access health records remotely, speeding up processes, avoiding duplication, and saving between 20 and 30 per cent in administrative costs (BCG, 2012).

According to (CAST, 2013) the already cited report of the project called Medicare, the results of the home telehealth pilot project demonstrated that home telehealth intervention had a significant and positive impact on clinical and financial measures: telehealth reduced the need for emergency department intervention, inpatient hospitalizations and the associated 
Medicare costs for HCBS/FE client and the patient perceptions of the intervention remained positive and stable over time (Newman \& McMahon, 2011).

One of two implemented projects in Canada and Minnesota with COPD resulted fewer nurse home visits and hospitalizations for patients in the experimental group. In summary, six months of remote monitoring saved a significant amount of money per patient (15 per cent of the total cost for this service) compared to traditional home care (Maccallum \& Austin, 2000).

\subsubsection{Prevention of disease and early detection through eHealth}

Early detection and management of diseases, using data utilization and visualization, medical experts can find patterns and anomalies (Pantelopoulos \& Bourbakis, 2010). Health experts are also beginning to see how to apply data mining for early detection and disease management. The process of detection, management of diseases by visualization facilitates the job of medical experts to find out anomalies then simply observing a set of tabulated data. Using data manipulation through data mining is not considered harmful for human beings, but in-depth scrutiny of the entire process leads to a conclusion that it has harmful effects on people after being used in a long-term perspective (Grieco et al., 2014).

(Patel, Park, Bonato, Chan, \& Rodgers, 2012) state that one of the most exciting areas in the field of technology is the use of sensors and patient-doctor communication systems to reach early detection of changes in the status of the patient requiring clinical intervention. An example of the application of this technology is the clinical management of patients with chronic obstructive pulmonary disease (COPD). The main purpose in the clinical management of this disease is to achieve early detection of deterioration episodes. Deteriorations commonly referred to as breathing difficulties. On the other hand, potential patients are claimed to be workers who have static work during the day, especially office workers such as full-time programmers and drivers, who work $70 \%$ of the time without any physical activity. Patients can be monitored for issues related to psychological stress due to high workload, de-concentration and anxiety. This patients are individuals at an early stage of an illness or a less complex form but should prevent the aforementioned diseases by using eHealth opportunities (Fairburn \& Patel, 2017). eHealth systems help and have a particular 
impact on patients who work but do not have any ongoing treatment, and have medical prescriptions, should be checked by the doctor from time to time (Patel et al., 2012).

Therefore, based on the status report that is generated by the system continuously, early detection and prevention are very important in case of in these patients. Depending on the patient's profile, it is necessary to determine which monitoring technology will be selected, static or clustered sensors, mobile technology or PC, local server or cloud services, internal or external protocols, algorithms for communication and data analysis (cf. Big Data) (Mshali et al., 2018). To act effectively, doctors need to provide complete and accurate information about the patient. (Tresp et al., 2016) think the most promising feature of the Big Data approach is predictive analysis, which reveals the potential risks even before they occur during eHealth data manipulation. One way to address the problem of achieving early detection of worsening episodes is to detect changes in the level of activity performed by a patient and assume that a decrease in activity is an indication of the possibility of deteriorating clinical condition of the monitored individual. Development of intelligent sensors can be used to monitor physical activities of patients suffering from chronic obstructive pulmonary disease. On the other hand, clinical professionals require quick access to relevant data and they want to be confident that what they see on screen is a true representation of what has been said about the patient. Emergency access to health records is sometimes needed by caregivers, otherwise losing responsibility in the patient's normal care (Nadeem et al., 2015), (Ziefle, 2011).

\subsection{ICT Infrastructure for eHealth Implementation}

A systematic review of literature I have undertaken is broadly discusses researches related to tele-monitoring services, self-care and emergency treatment. The medical field needs to understand current technologies such as IT infrastructure, platforms, protocols, equipment, standards and possibilities. Benefits will be fully considered and covered by many articles, case studies, industry news and reports. The realization of ubiquitous computing requires the collaboration of many ICT areas. Its implementation depends on several factors such: specific application and devices (smaller, lower power processors), physical and semantic embedded systems, ubiquitous connectivity, interoperability (seamless networks, self- 
configuring), intelligent systems including sensor networks, context awareness, data handling, security and reliability (Ziefle, 2011), (Albahri, 2018). Researchers identified the eHealth infrastructure components based on demand to support the distribution of structured and meaningful health information across the geographic and the health sector boundaries, to support improved or new ways of providing care services. via high-speed data connections (WiMAX, 5G) with health information records, authentication and authorization services (Jovanov \& Milenkovic, 2011), (Oleshchuk \& Fensli, 2011).

Other investigations (Oleshchuk \& Fensli, 2011), (Ziefle, 2011) recalled some difficulties that may occur during the collection of data through medical devices. They concluded without a good technological infrastructure, the nurses may have problems to use multiple systems to look up information they need for the interventions such as patient history, medications, or to document the intervention after each call from patients. Summing up the results of these projects, the conclusion with certainty the ubiquitous computing requires the collaboration of many ICT areas. (Jovanov et al., 2005) expressed, the good technical infrastructure implementation depends on several factors, as it was mentioned above.

Referring to works of (Aminian, 2013), (Rolim et al., 2010) as far as technological equipment is concerned interoperable (ubiquitous) networking aims to provide seamless communications between persons and objects while they move from one location to another. Ubiquitous networking supports three types of communication: (1) person-toperson communication: persons communicate with each other using attached devices (e.g., mobile phone, PC), (2) person-to-object_communication: persons communicate with a device in order to get specific information (e.g., IPTV content, file transfer), and (3) object-to-object communication: an object delivers information(e.g. Sensor information) to another object with or without the involvement of persons (Salem et al., 2015). (Chen et al., 2011) believe still many challenges need to be addressed, especially broad bandwidth and energy efficient communication protocols, interoperability between body sensor network and other wireless technologies, and the design of successful applications (Alemdar \& Ersoy, 2010). 


\subsection{Data Standardization}

The information system is required to provide quick access to patient registration, patient is health profile and patient's medical history when and wherever a problem occurs. The patients profile data are essential in order to find and provide access to a patient's electronic health information. Electronic Health Record use a compilation of clinical practices, such as clinical data repositories, nursing documentation systems, clinical decision support systems, laboratory information systems, order entry, pharmacy management systems, physicians' documentation and diagnostic information systems. Information can be drawn from multiple sources while being managed, shared, and controlled by the individual (Meingast, Roosta, \& Sastry, 2006).

Referred to the studies about HER (Adebesin, 2013a) (Ziefle, 2011) it is an electronic record of health information of an individual that conforms to (inter-)nationally recognized interoperability standards and can be created, managed, and consulted by authorized clinicians and staff across more than one healthcare organization.

In three case studies (CAST, 2013) authors conclude that selecting a telehealth solution, the integration of telehealth data into the electronic health record (EHR) must be taken into consideration, but this is not trivial. Given that use of EHR is a new area, the staff is required to be familiar with the system and trained continuously in order to be informed about the technology. The worrying issue is security, namely protecting user information from unauthorized access. Also, privacy and confidentiality including the right of the user to control the collection and dissemination of personal information. In terms data management there are certain standards that must be followed as the System Health Level 7 (HL7) or ICD9 (Adebesin, 2013b), (Pedace et al., 2017).

(Piho, Tepandi, Thompson, Woerner, \& Parman, 2015) draw attention to the objective of HL7 Version 3 that aims to provide a multifaceted framework for the respective events, data elements and messaging. HL7 uses, in principle unique object identifiers and allows the control of sending messages on time.

(Piho et al., 2015) points out HL7 CDA (Clinical Document Architecture) is an XML based markup standard intended to specify the encoding, structure and semantics of clinical 
documents for exchange. Multiple standards exist in healthcare that is used in eHealth applications and interactions between humans, devices, processes, and applications. These standards can be classified in multiple classes including data standards, message standards, document standards, process standards.

(Peterson et al., 2016) also mentions the World Health Organization has recognized the importance of health data standardization that can be syntax-based, semantics-based, relationship-based, purpose-based, and classification-based. International standard codes for diagnoses (ICD) are used in electronic health information records (Health Level System 7), and IEEE 11073-20601-2008 standard also stands for the appropriate use (Peterson et al., 2016). Standard addresses need for an openly defined, independent procedure for converting the information profile of personal health devices into an interoperable transmission format, so the information can be exchanged to and from personal telehealth devices and computing engines. The organization recognizes that implementing health data standards to achieve interoperability at all levels of healthcare delivery is not an easy task, despite numerous efforts (WHO, 2006). Interoperability is widely recognized as an essential element to achieving the full potential of seamless data exchange using information, communication

technologies (ICTs) and medical devices in support of health systems (Adebesin, 2013b), (Hammond, 2010) . With reference to the work of (Meingast et al., 2006) complying to standards is an attribute of the system that should be valued, so the eHealth solution should fit in the trends of technological innovations that may occur in the future.

\subsection{Privacy and Security in eHealth}

eHealth can be effective only if patients can trust the eHealth websites they access. eHealth data is usually stored by different entities at different places of healthcare IT systems (Ziefle, 2011). For instance, researchers need health data for clinical research, doctors exchange health data for a second opinion, and patients send their medical histories to doctors to get the right treatments. The most important security requirements for eHealth applications are described like availability, confidentiality, integrity, anonymity. User awareness based on a secure system built on three main functions: data protection on the device, secure authentication and data encryption (Wetzels et al., 2018) . 
Recently the healthcare security threats started being taken into attention because of the great impact that they have in our lives. Lots of people usually underestimate this impact because there is a great lack of user awareness, hence this is one of the fields that really needs more attention and understanding. Healthcare data is extremely valuable to criminals. For example, if somebody takes our banking card data they will make money from our card information, but if an attacker steals our healthcare information they can impersonate our lives entirely and do much more than just taking our money, they could take our life (Rahmani et al., 2018)

(Al-Janabi, 2017) mention security and privacy protection are of particular importance in the eHealth system, where confidentiality should be treated as important. Since healthcare data is communicating through wireless networks, efforts must be made to maintain its confidentiality and privacy. Privacy includes the right of users to control the collection and dissemination of personal information, while security is the protection of user information from unauthorized access. To assess the eHealth security architecture, engineers need to focus on the latest perspectives and assess the security challenges, we face in each of the key areas, including end-users and their access to internal networks, cloud services, partners, internet service providers, health system data held on web platforms, and implications that may occur from the standards applied to wireless devices (Meingast et al., 2006), (Rahim et al., 2018). The forthcoming evaluation of any eHealth solution is intrinsically linked to the successful deployment of a secure and privacy-preserving eHealth infrastructure.

Privacy and security during building standards have focused on communicating all actors in the system such as Endpoint Devices Security, Network Security, Application Access Security, Data Storage Security, Federated Secure Access to Partner Cloud Service (Al Ameen et al., 2012). Many project and author still need to cope with this challenge and especially the inerrability between the equipment and the standards is the main problem. Act of 1996 (HIPAA) set up many different rules to be followed by doctors, hospitals, healthcare organization and other health-related professionals (Alves, Marquezan, \& Granville, 2015).The Act requires comprehensive data security measures for data administration policies, data safeguards, and supporting systems. Based on social issues of WBAN systems including privacy, security and legal issues, due to the communication of health-related 
information between sensors and servers, all communication over WBAN and Internet should be encrypted to protect users' privacy. Legal regulation will be necessary to regulate access to patient-identifiable information .

\subsection{Types of Hardware Used for eHealth Systems}

Definition of the terminology associated with the advanced technology is necessary in order to understand the operation of state-of-the-art devices and systems, which are now dominating and revolutionizing eHealth systems. (Pahl et al., 2015) discuss in detail that ICT infrastructure needs to be built carefully on how devices are connected to the ecosystem in healthcare. The tendency is choosing the most advanced communication technologies inside and outside the system, choosing the right communication between sensors and data storage devices, communication between mobile devices and the system using the latest technologies like 4G, 5G (Oleshchuk \& Fensli, 2011).

An assessment should also be made based on (Santos et al., 2016) research, which will also include exploring the underlying widespread network features starting with IP connectivity, intelligence labelling, radio frequency, IoT and IPv6. The acceptable computer solution on the network part will greatly affect the functionality among the variations in the equipment that the system will be built up .

Patients use the smart systems which monitor, collect or process data on remote/wireless platforms (Jovanov \& Milenkovic, 2011), (Pantelopoulos \& Bourbakis, 2010). Wireless sensor area network, wireless body area network, and personal area network are terms for wireless wearable telehealth monitoring systems, they collect vital signs from patients by attaching sensors directly to the body (Chatterjee, Das, \& Sing, 2014) list many challenges arise when implementing a WSSN for structural monitoring that is not present in wired systems. The design of a wireless monitoring system, employing a large number of sensors, must account for the limited resources of smart sensors, time synchronization, limitations in the types and quality of sensors available, data loss associated with RF communication and network fault tolerance (Jovanov \& Milenkovic, 2011). Based on wireless communication protocol standards wearable health monitoring systems use a different protocol such as Bluetooth, Zigbee, Infrared Ultra-wideband (Ziefle, 2011). The authors warn the main 
limitation of using body sensor whether the system is capable of monitoring only real-time patient's status and is not able carrying out professional analysis and giving instruction. Therefore, data analysis with experts' experience needs to be further studied to provide more useful information. According to the user query, some users are not very enthusiastic about the idea of continuously wearing a monitoring device. The reason could possibly be the overall discomfort caused by the belt. In the future, the focus will be given on how to improve the wearing experience of the sensor (Myers, Grant, Lugn, Holbert, \& Kvedar, 2006).

(Fagerberg \& Kurkinen, 2012) recall the emergence of Patient Monitoring System applications that can address key issues or challenges such as worldwide increasing healthcare-related costs, the increasing number of an ageing population, the high usage of ubiquitous devices (smartphone, tablet, or PC) in daily life and enhancing overall healthcare delivery. Since professional equipment always requires operational skills and limits the patients' mobility, it is unsuitable for daily monitoring of chronic disease patients. Such situations bring challenges for continuous monitoring and mobile health (Lorenz \& Oppermann, 2009). Central to mobile health applications is the tracking of body functions and physical activity, commonly referred to as self-tracking or quantified self. Self-tracking activities are linked to the empowerment of patients through the promise of offering new knowledge about the body, which might lead to new practices, bodily changes, and better health outcomes (Greenspun \& Coughlin, 2012). Thinks this is still an open research question need to be addressed and the opportunity for research to address a particular question such as do wireless remote mobile patient monitoring systems make a difference to the patients' well-being? To answer this important question, many researchers have included the views of patients as well as of medical professionals at every stage of the design and development (Fagerberg \& Kurkinen, 2012). The acceptance of any system in the healthcare industry depends on user awareness and acceptability. The adaptation of a device within the clinical field is diminished if it is negatively perceived (Lorenz \& Oppermann, 2009).

\subsection{Best Practice and Relevant Projects}

The literature review presents an overview of researches and reports that seeks to understand deeper the implemented health systems in different countries. Looking at case studies, the 
practices of different countries give the opportunity to research, analyze and compare the innovation, development, benefits, strength, weakness, advantages, and disadvantages of these systems. By understanding and analyzing these factors, we will be able to create a suitable idea for a system of healthcare in Kosovo.

Knowing that health sector is faced with expensive and complex services, which have a major impact on the economy, and the everyday lives of peoples, the United Kingdom is one of the first countries, which has always been seeking improvements of the national healthcare system. A very important role in improving the NHS system was the technology that has been becoming more and present in these systems. In 1998 the representatives of National Health Service (NHS) published "Information for Health: An Information Strategy for Modern NHS 1998-2005", a strategy that aimed at engaging in the development of electronic health records, patient data recording, technical infrastructure, and guidelines for improving telemedicine use (Grosios, Gahan, \& Burbidge, 2010). Thus, in 2002, the National Program for IT (NPFIT) was established, where key points in this program were: providing accurate information to patients at general points of health services known as GP, reducing the risk of treatment errors and assisting healthcare staff to be fully informed about treatment decisions, prompt and accurate transmission of information such as descriptions and books in different NHS locations, increasing efficiency in the NHS by reducing time spent on retrieving data of patients by staff. One of the key goals of UK is to have a completely electronic healthcare system by 2020, leaving behind traditional forms of data retention in the paper (HMSO, 2009). 


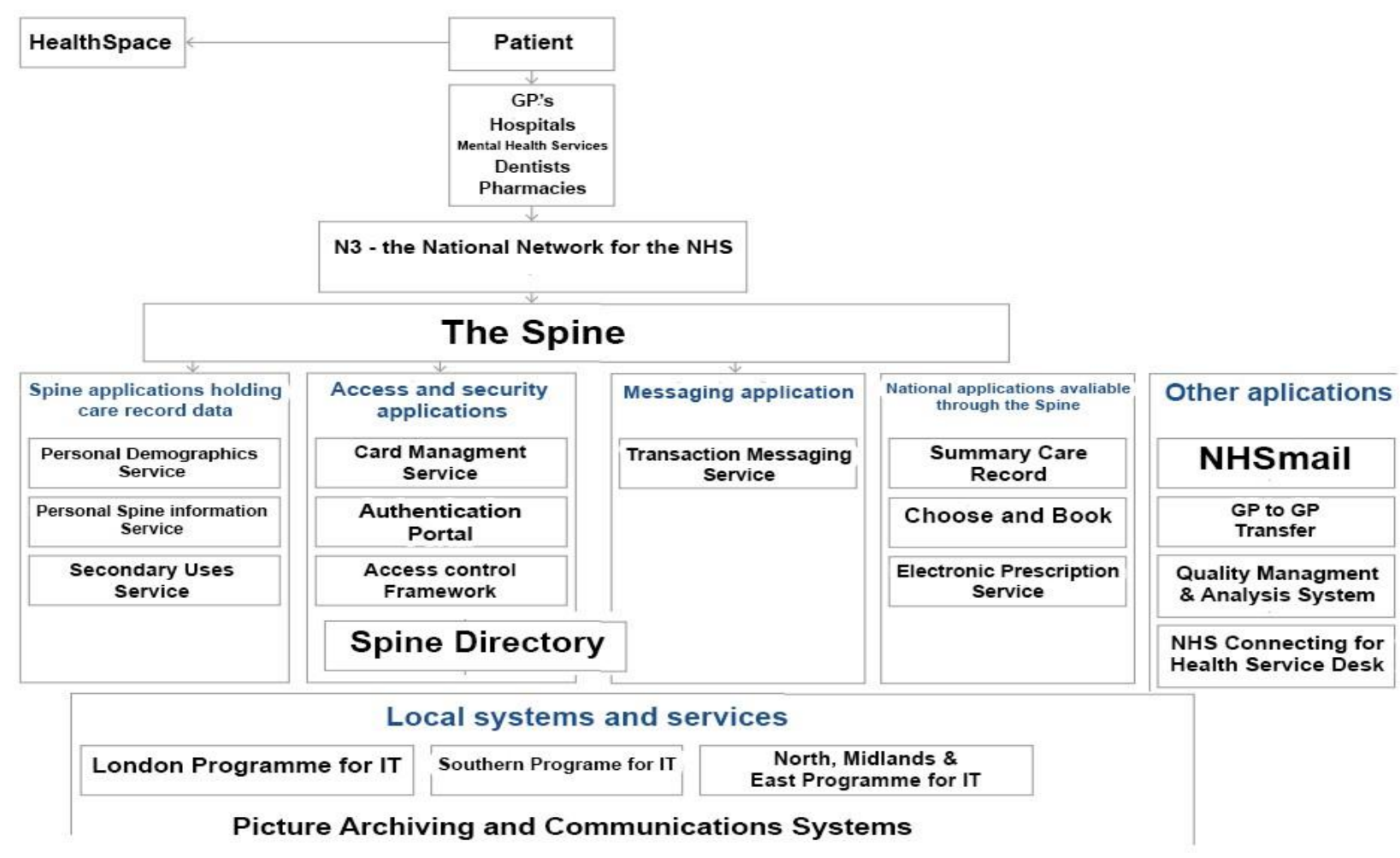

Figure 4. Structure of main systems and services in the UK (HMSO, 2009)

The use of healthcare applications on mobile devices is playing a special role, and according to the NHS, it is a part of the game, in realizing the goal of full electronic data handling to be achieved by 2020. Mobile devices we mean, any device that can be used for storage, transport, and access to data and health applications that are used by mobile devices are known as mHealth (HMSO, 2009), (Grosios et al., 2010).

Mobile devices in healthcare organizations provide access to technology for information access and electronic health data in the network as well as their transfer to other locations. These devices, especially smartphones and tablets, are used by NHS healthcare organization staff who access the organizations' applications, such as emails or in more specific cases with the approval of authorized persons, access to other data. Since 2015 healthcare organizations in the UK also offer some opportunities for patients who can perform online healthcare services through mobile devices. These services enable patients to reserve or cancel appointments at the time that suits them, the message of recurring descriptions online, see some of their online information recorded by the GP. The following five types of mHealth 
applications have been distinguished: those providing access to health records; consumer versions of existing medical devices; those for monitoring and management of a condition; fitness trackers and wellness coaches; and those related to diagnosis or treatment. mHealth is often combined with eHealth; a mobile device that collects data and presents them on the device, and a website that can use the data for monitoring or detection. It is noteworthy that this rapidly evolving field has many possible legal and practical stumbling blocks. Issues around qualification as a medical device, data protection, privacy, and 'medical' responsibility are important and need to be addressed with proper legislation and practical implementation (Grosios et al., 2010; National Audit, 2008).

Two projects, Health Gear and CodeBlue (Al Ameen \& Kwak, 2011) aim to provide affordable continuous monitoring of a person's health-related issues. The major focus is given to the cost effectiveness and power consumption of devices used.

The LifeGuard project (Patel et al., 2012) which ran for astronauts in the first place, was also used to monitor the signs of the human body. The system consists of several components, the sensor interface can support different types of sensors such as ECG, breathing, pulse oximeter, and blood pressure.

The intelligent AlarmNet project was a prototype of a wireless medical network consisting of five components. The cellular network of body sensors is responsible for physiological monitoring and follow-up functions (Patel et al., 2012), (Alemdar \& Ersoy, 2010).

A project based on specific patient requirements, called MobiHealth was one of the earliest projects that integrate all sensor devices as body sensor, as PDA phones and the smartwatches a person carries during the day. Based on the literature review of mobile technologies we still did not find a version which integrates the entire eHealth ecosystem, this is also a future research topic (Ziefle, 2011), (Al Ameen \& Kwak, 2011). 


\subsection{Summary}

We see that ICT has covered all aspects of Life, especially healthcare. Various technological devices have found great application in the treatment of patients and administrative processes in healthcare. Process digitization has affected many areas ranging from health treatments, real-time monitoring, and online appointment to doctor appointments, to payments for health services. Most of them can be performed using electronic platforms. The benefits of ICT implementation in healthcare are many, including easier access to information, better organization of resources, cost reduction for patients and health staff, more efficient treatment by medical staff. In this process, benefits and challenges every time is evident in health systems, starting from data security, interoperability between different technologies, standardization of different technologies in health-ecosystem are always evident. The medical field needs to understand current technologies such as IT infrastructure, platforms, protocols, equipment, standards and possibilities. The vision of any eHealth project reviewed in the literature aims to integrate information technology into a medical ecosystem. The correct selection and integration of communication technologies is still an open issue and needs to be addressed in the context of eHealth system information. 


\section{Role of Monitoring in eHealth}

\subsection{Health Indicators}

From the perspectives of improving performance outcomes in the health system also indicators are important, as they represent important aspects of the health system performance as well as they are the basis of quality assessment of health services. The purpose of these indicators is to measure progress in achieving results and assessing their impact. WHO suggests the following leading indicators in the EU and Balkan countries?

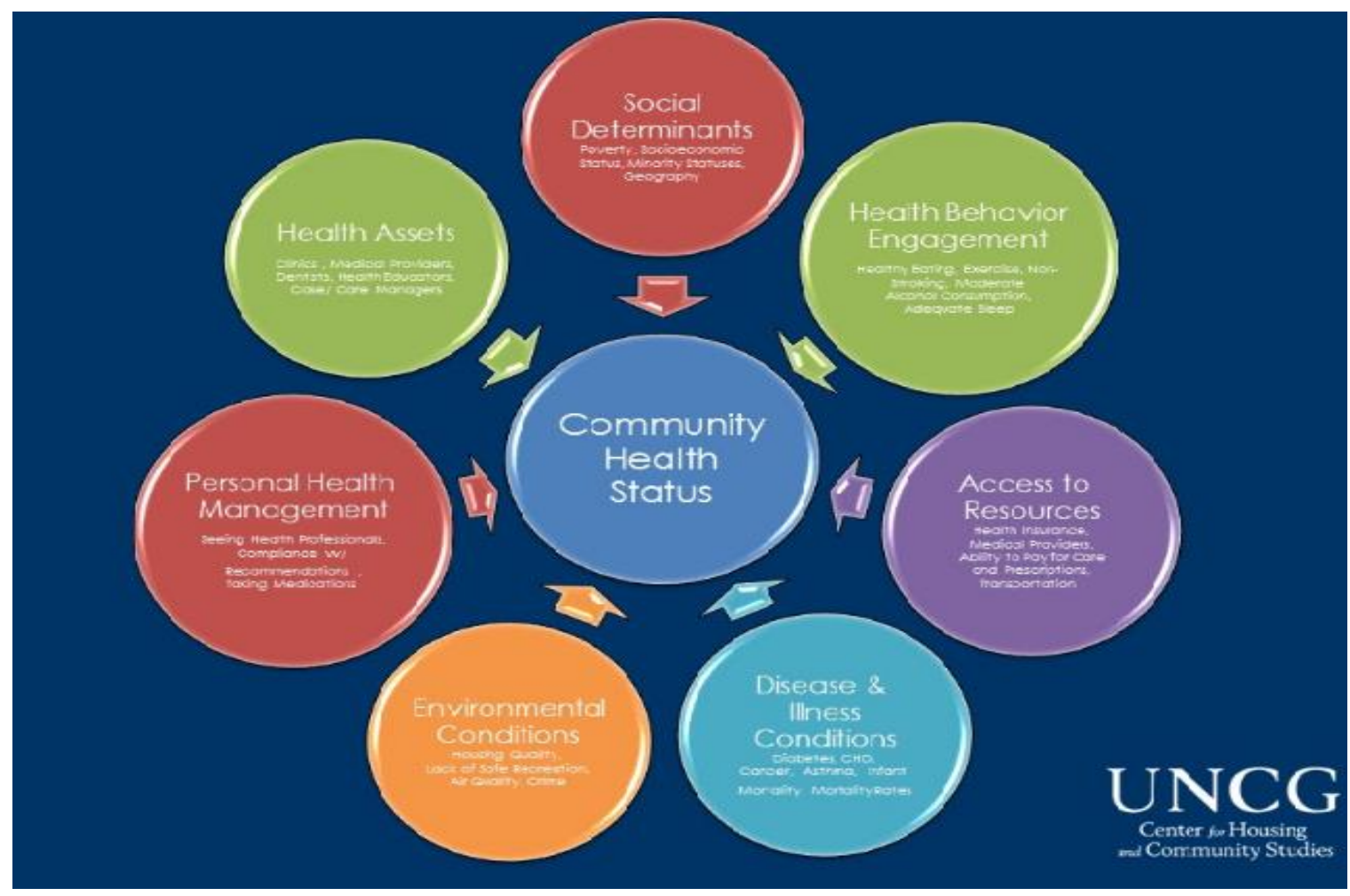

Figure 5. Health indicators suggested by WHO

\subsubsection{Health status}

One of the indicators that shows the performance of the health system is the indetermination of the health status of the population, which can be measured by pathological and clinical measures and is usually provided by doctors or using measuring instruments. One of the health measuring indicators is life expectancy and mortality of the population. Based on data from the EU Statistical Office (Eurostat), the World Health Organization (WHO), the World Bank and the Organization for Economic Cooperation and Development (OECD) in Europe, 
including the Western Balkans life expectancy reaches average up to 81 years. While the main causes of deaths in the European countries, including the Western Balkans, remain cardiovascular diseases (over 1.900.000 cases in 2015) and cancers (1.320.000 cases), which together count for over $60 \%$ of all deaths. In the (WHO, 2015) below statistics for 2015 are presented based on Eurostat mortality data for the of the population in EU countries, separated by gender and disease, (WHO, 2016a), (Fehr et al., 2018b).

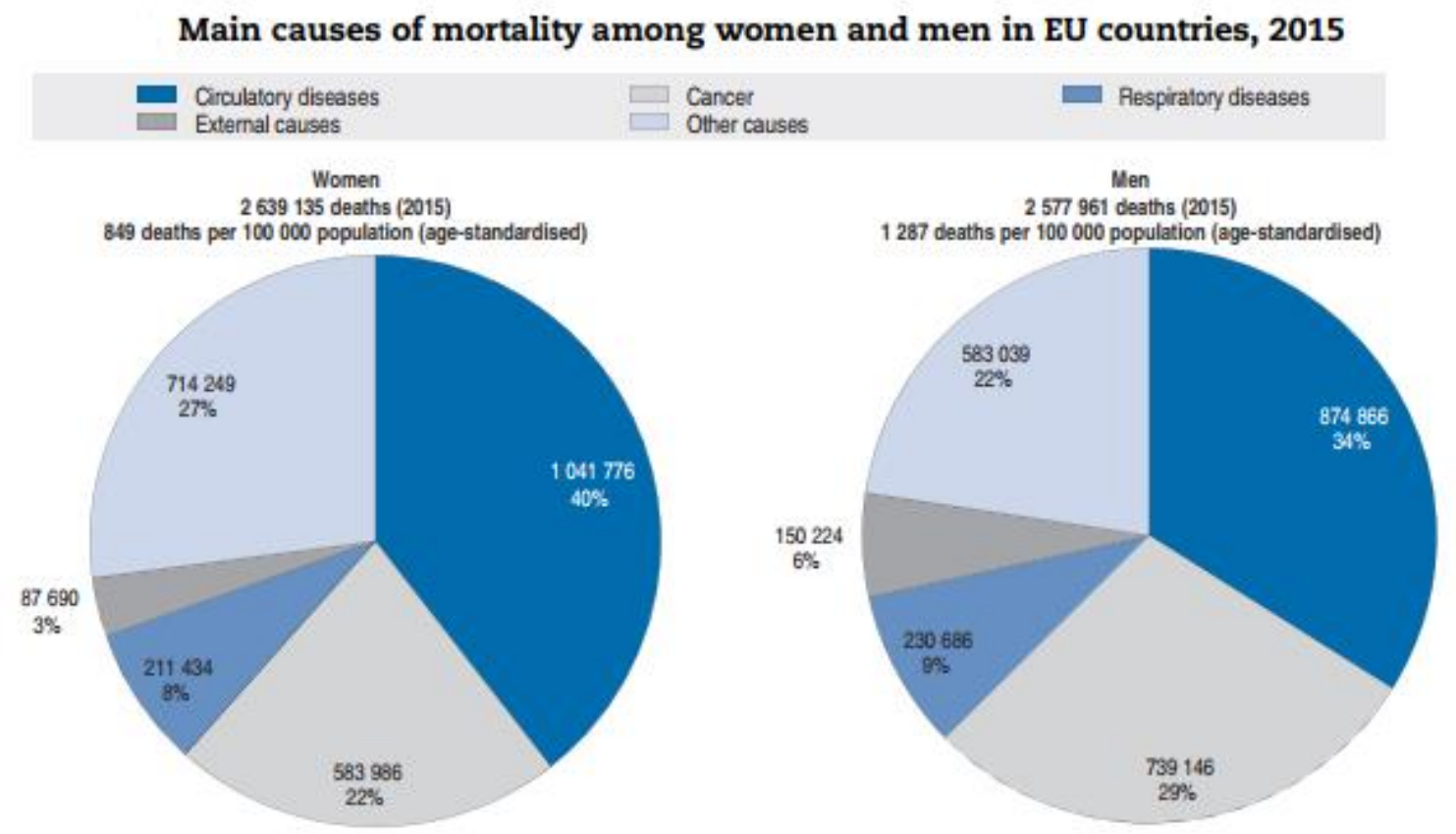

Figure 6. Main causes of mortality among women and men in EU countries, 2015 (WHO/EU, 2016)

As far as Kosovo is concerned, according to the Kosovo official statistical reports (KAS, 2013) the most common causes for mortality of patients, related to cardiovascular and oncological diseases, in 2014, 3.996 patients died from cardiovascular diseases, while 1141 patients died in cancer. 


\subsubsection{Healthcare risk factors}

Patients' health is affected by various risk factors, including smoking, alcohol consumption, and obesity. Other factors are related to environmental factors such as air pollution and extreme weather conditions. Recent estimates indicate that around 790.000 people in EU countries died prematurely in 2016 due to smoking, alcohol consumption, unhealthy food, and lack of physical activity.

Exposure to serious air pollutants is estimated to have caused the deaths of some 240.000 people in 2016. In the Balkans, the main risk factors are uncooked food, polluted air. Kosovo and North-Macedonia are ranked as the first two countries with the most polluted air in the world (European Commission\&OECD, 2018).

Based on official statistical data outdoor air pollution is the most influential factor in Kosovo, estimated loss cost €97.6 million per year, causing 852 premature deaths, 318 cases of chronic bronchitis, 605 hospital admissions, and 111.900 emergency visits. Lead contamination is the second factor, estimated to cost $€ 67.9$ million, mainly due to gas emissions from lead-free and zinc mines and processing facilities. In general, the polluted environment has a very negative impact on the health status of Kosovo's population, at a cost that is higher than all the investments made for health in Kosovo each year (KAS, 2013).

\subsubsection{Effectiveness: quality of care and patient experience}

The effectiveness in improving the quality of patient care and patients' experience is based on indicators of mortality avoidance and reduction of premature deaths (OECD/EU, 2016). In 2015 more than 1.2 million people in EU countries died early from diseases and injuries that could be avoided through more effective public health or healthcare policies. The main causes of avoidable mortality include chronic heart disease, lung cancer and accident. Health systems in most European countries have made progress in treating cancer through implementing screening programs and providing effective cancer care (WHO, 2015). Healthcare should be provided by placing patients at the center as well as publishing experiences reported by patients earned during their contact with doctors. In most countries, patients' experience reports appear to be limited, while available data indicates that patients generally report positive experiences. The below shows Eurostat data for the leading causes 
of preventable and amenable mortality in the European Union, in 2015 (European Commission\&OECD, 2016), (European Commission\&OECD, 2018).

Leading causes of preventable and amenable mortality in the European Union, 2015

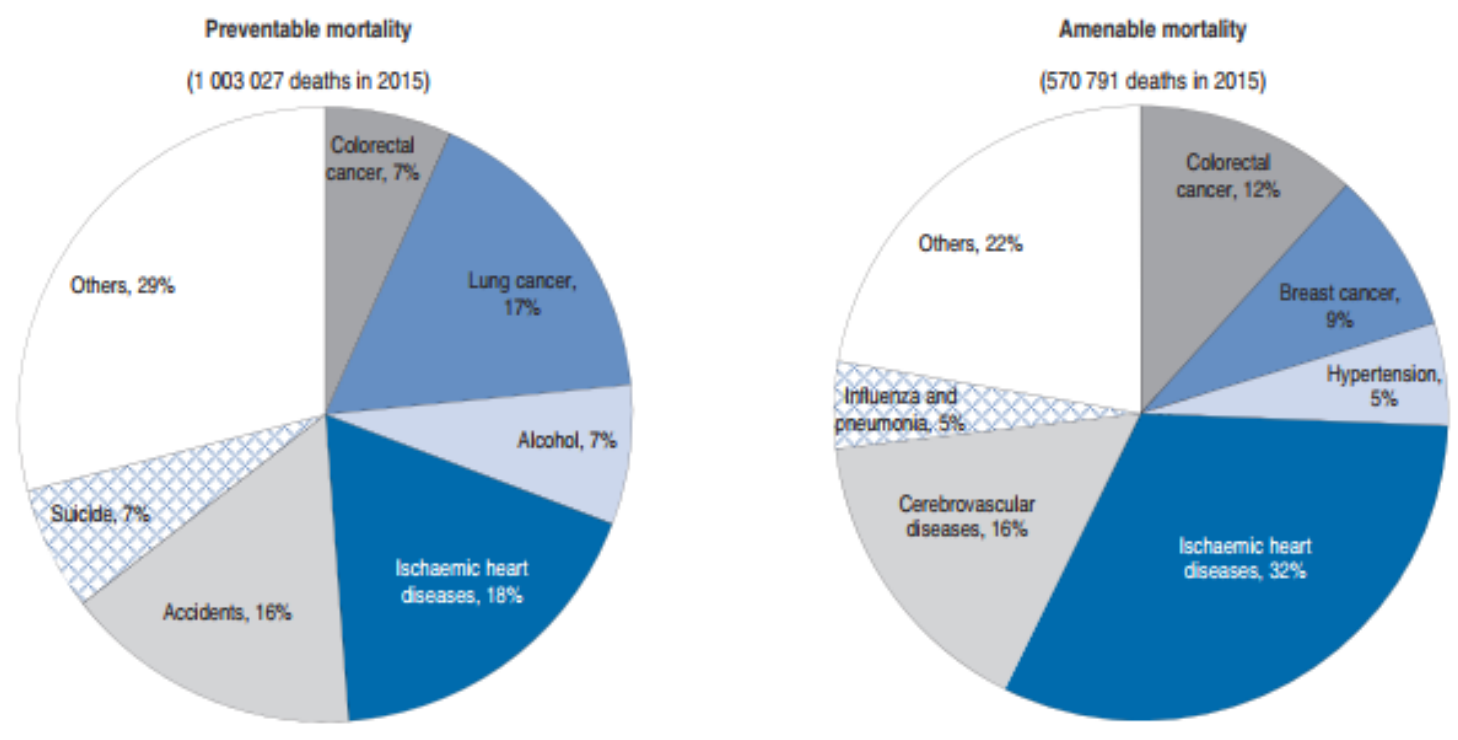

Figure 7. Leading causes of preventable and amenable mortality in the EU, 2015 (Fehr et al., 2018b)

In Kosovo, capacity utilization has reached a negative peak and the quality of health services needs to be improved, especially regarding hospital infections, the use of standardized guidelines, clinical protocols. The different age groups, in particular, the middle and the old age groups, which represent predominantly a large part of the population, have limited access, and for them, their diseases present a huge economic burden, too. Analysis of the health status of the population shows that the number of deaths in 2016-17 in Kosovo was 421.9 deaths per 100.000 inhabitants (MoH, 2010c), (KAS, 2017b).

\subsubsection{Health costs and funding}

One of the key indicators influencing the health system performance is the funding of the system. According to data from OECD, Eurostat and WHO in 2017 healthcare spending in the European Union was $9.6 \%$ of gross domestic product, this funding remained unchanged from the previous two years. In the majority of the European countries, including Western Balkan countries (see the Figure 8 below) most of the funding is oriented towards curative and rehabilitation services, while in most countries, pharmaceutical spending constitutes a large part of the health system funding. However, in Western Balkan countries, the main 
challenge is the operating of the health insurance system. It is important that most of the expenses for health services come from patients' personal finances. The Figure 8 below shows the expenditures in the percentage of GDP for 2017 (Fehr et al., 2018b), (European Commission\&OECD, 2016).

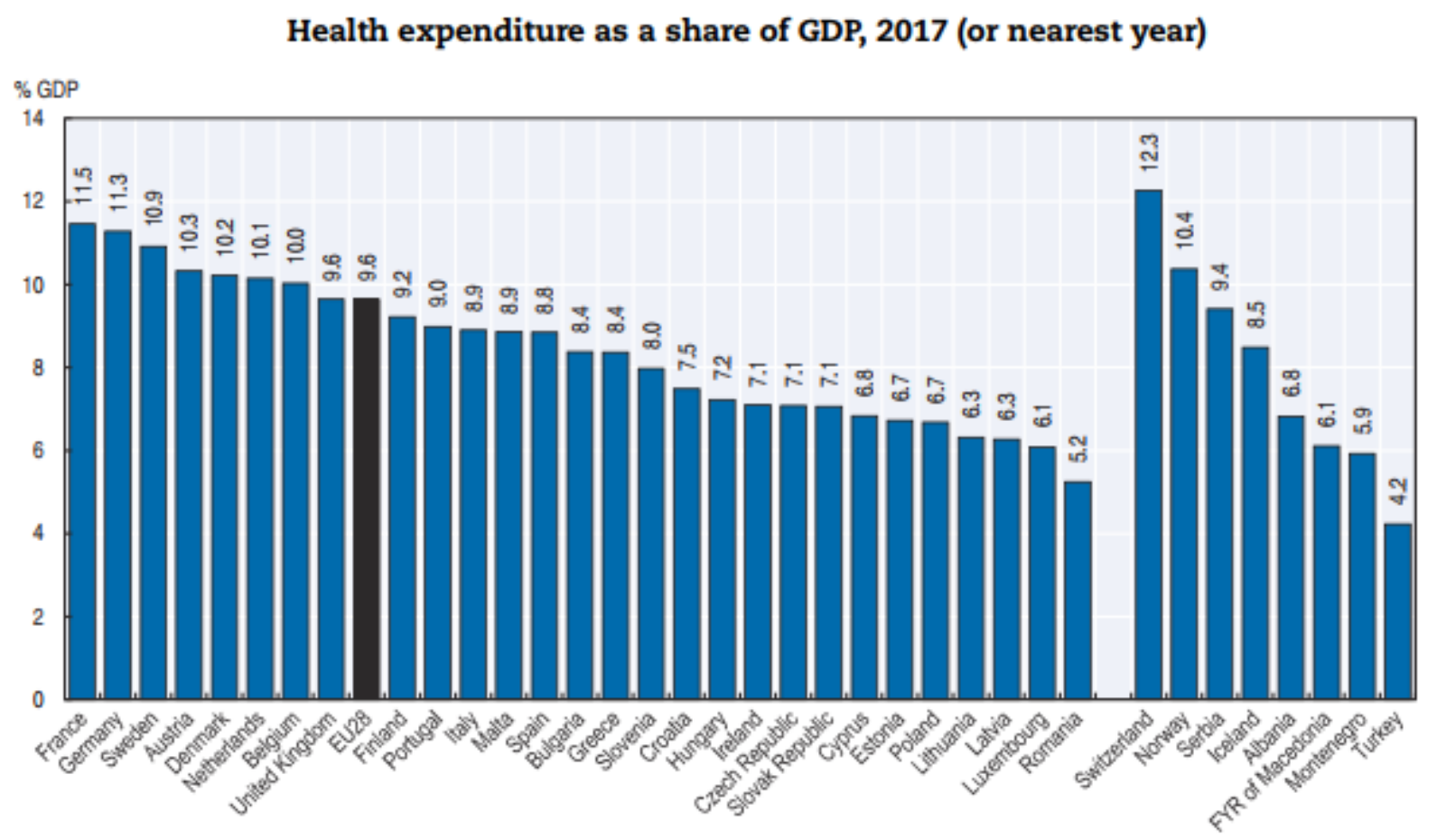

Figure 8. Health expenditure as a share of GDP, 2017 (Fehr et al., 2018b)

In Kosovo, the environment also affects health and life expectancy. The World Bank study shows the cost of influencing environmental factors in healthcare, including water and sewage, is $€ 222.9$ million per year, which has a very negative impact on the economy (5.3\% of GDP in 2010). In order to address this problem, the Ministry is in the process of preparing an assessment of the environmental impact on health with donor support (KAS, 2018).

\subsubsection{Use of services}

Patient access to health services is the main objectives of any healthcare system because if access is limited, health outcomes will be poor and cause large inequalities in the system. There are various reasons of the limitations of access to healthcare; the main reasons are the unbounded costs of the patient for health services, long distances from healthcare centers, and the long waiting time for treatment. (Imasheva \& Seiter, 2008) analyzed recent data from 
low-income households and concluded that unmet needs for care compared to high-income households, explained mainly by financial reasons. Another factor affecting the nonavailability of health services is the lack of doctors, which according to the data has increased over the last decade in most European countries, particularly in the Western Balkans. In the Figure 9 below, coverage of healthcare services and private health insurance for 2016 in EU countries, including Western Balkan countries, has been increased (European Commission\&OECD, 2016), (European Commission\&OECD, 2018)
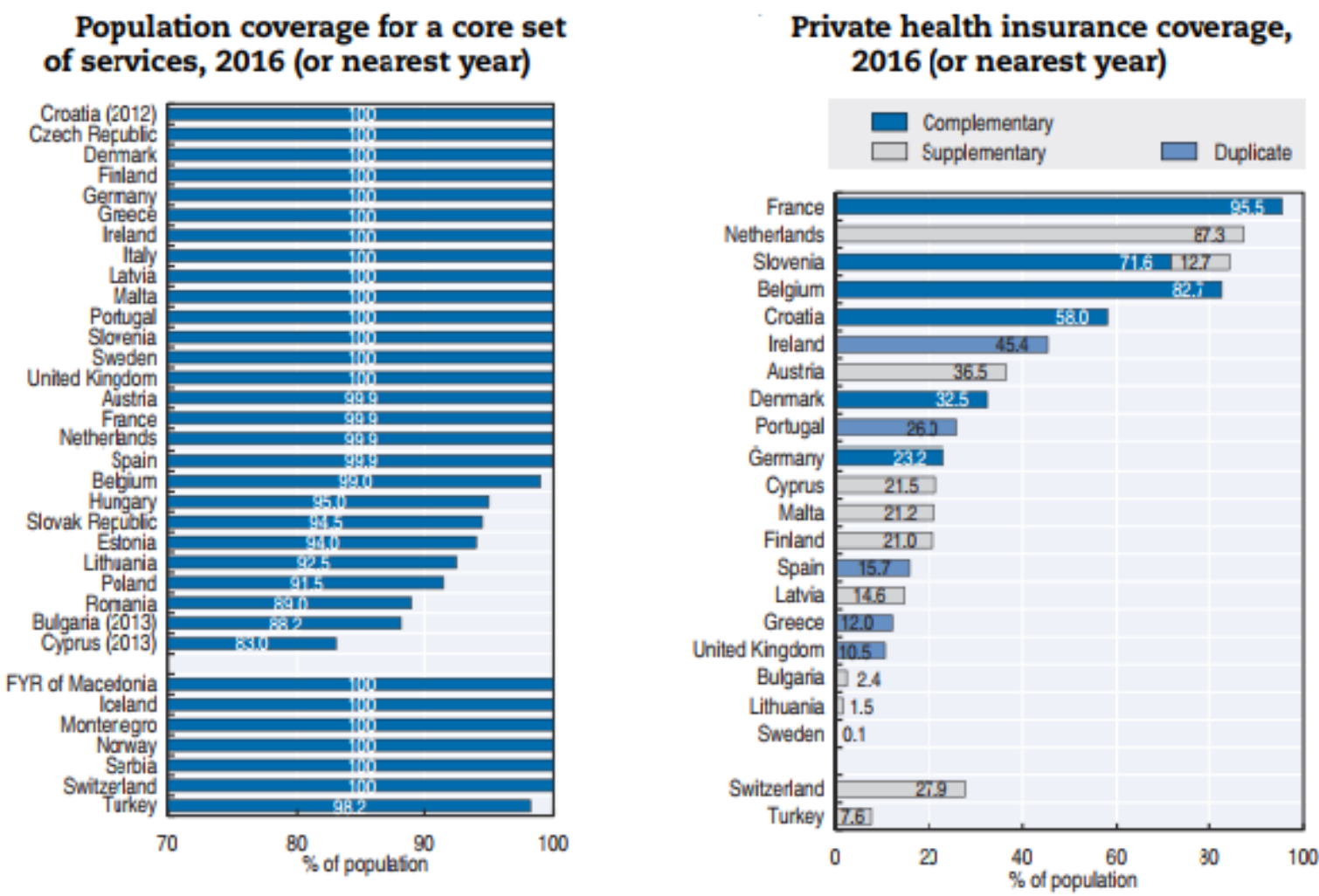

Figure 9. Coverage of healthcare services and private health insurance for 2016 in EU \& Western Balkan countries

\subsubsection{ICT in healthcare}

The efficiency of health services is also closely related to the use of ICT, which according to WHO, is one of the main indicators in the improvement and elasticity of health systems. This creates opportunities for efficient adaptation of health systems with changes in economic, technological and demographic environments. ICT provides great opportunities for health services, empowering patients to access their health data electronically, and enables data 
exchange healthcare providers across all EU countries. WHO reports the use of eHealth and ePrescribing has begun applied in most EU countries as a result of the implementation of ICT systems. Good practices are contributing to the improvement of the health system in developing countries, especially the Western Balkans. Based on EUROSTAT data the Figure 10 below shows the percentage of use of electronic health data from 2012 to 2016 (OECD/EU, 2016).

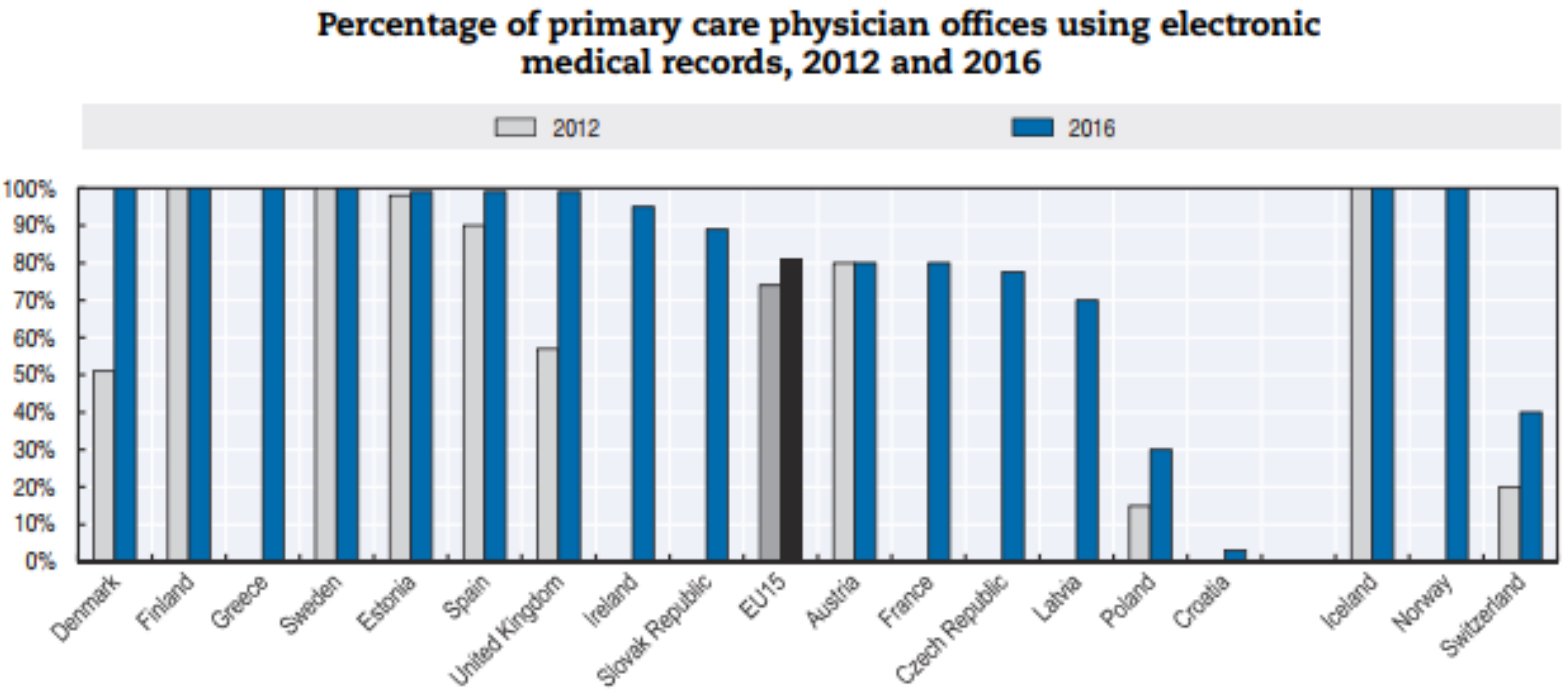

Figure 10. Percentage of use of electronic health data from 2012 to 2016

\subsection{Tele-monitoring and eHealth}

Tele monitoring is considered a promising and effective way to provide more integrated health services in the home of patients. As outlined in the literature, tele monitoring requires the use of technology and equipment, as well as the collection and transmission of measured and subjective clinical data at distances between patients and a healthcare provider through electronic information processing. (Ellingsen, Christensen, \& Silsand, 2014) gave a definition of tele-monitoring that means providing remote health services by using a variety of technologies, and this includes mainly two areas: infrastructure and services. Telemonitoring consists of several components such as network, computer equipment, smart devices, telephone or video devices to support diagnosis consultation with patients under monitoring (Song, Sarver, Aranki, Bajcsy, \& Javey, 2016) . 
Health managers and policy-makers need to consider how tele-monitoring reconfigures existing practices and relationships between different health professionals collaborating in various health organizations. Tele-monitoring can be beneficial for citizens (patients) and care professionals, but also for health organizations and public authorities. When effectively implemented tele-monitoring provides more personalized healthcare, the healthcare is more citizen-centric, also positively influences the reduction of diagnosis errors and the length of hospitalization (Chaudhry et al., 2011).

\subsubsection{Advantages of Tele-monitoring}

The benefits of tele-monitoring, as mentioned in the literature, are in many aspects: costeffectiveness, the better use of medical staff, easier access to health services. The easier access especially stands for rural areas where healthcare is less developed, and patients have difficulties to reach professional help in emergencies services. Both in rural and urban locations healthcare centers have implemented tele-monitoring services improved the quality of health services and promoted the prevention of diseases (Stachura \& Khasanshina, 2007) Tele-monitoring also plays an important role in treating patients with diseases requiring longterm treatment, enabling the patient to be monitored occasionally by healthcare staff. Telemonitoring creates the possibility for patients and healthcare staff to take precautionary measures to prevent lethal incidents that for most countries in the world still remain one of the key health problems, especially in case of COPD, cancer and diabetes. Treating these three diseases through tele-monitoring offers patients the opportunity to have rapid and efficient treatment (Marcos, Maldonado, Martínez-Salvador, Boscá, \& Robles, 2013).

\subsubsection{Challenges in tele-monitoring}

While technology improves processes and spares, time spent on administrative tasks or consultations with healthcare staff, high-quality technology solutions will require a restructuration of IT. This restructuration can lead to increased costs of implementation of a health system, however, it is counter-balanced with a higher return on investment on a longer time (WHO, 2011).

The main challenge remains the privacy and data security. Since technology not only supports digitalizing data communication processes, it also allows passing data through 
various digital platforms, this is from where the patient data security risk stems. Data security along with data reliability is a key element that hinders the tele-monitoring process and has opened up a new challenge for engineers who recommend a technologically dependable infrastructure (Stachura \& Khasanshina, 2007). Most tele-monitoring devices are still not standardized and incompatible with each other's, so in most countries, systems developments are based on local requirements and strategies(Christensen, 2018), (Ellingsen et al., 2014).

As a result of endangering patient data security and privacy, the EU legislation (GDPR) became mandatory for all member states from May 25, 2018.The GDPR implementation challenge will set a higher level of data protection for relevant public institutions in member states, particularly for institutions and persons responsible for collecting, processing and maintaining patient data, like health insurance companies, pharmacies, and hospitals. (Yuan \& Li, 2019).

The implementation of web and cloud-based tele-monitoring platforms (not exclusively) has created new challenges for the design and implementation of policies coping with cybernetic attacks on electronic medical records (EHRs), medical records of patients that are readily available to health practitioners through the web; websites or applications, which are also a favorite target for to malicious people or hackers. In developing countries, there may be a challenge for both the management and the legislation addressing issues related to compromising patients' data (Al Ameen et al., 2012), (Shen et al., 2018).

As technology development assumes that doctors, nurses and other staff members should be fully trained in all systems planned to be implemented. the technological renewal invokes new competencies to ensure coping with changes and best enforcement practices. Therefore, this also challenges that policy-makers who should address this requirement in their strategies.

Given that tele-monitoring is a new field, there is an urgent need to enhance the education of otherwise highly trained professionals, especially developing countries such as Kosovo, in line with technological developments and the implementation of new communication platforms in the health services. The additional training creates additional costs and, at the 
same time requires additional investment in laboratories (Anker et al., 2011), (Stachura \& Khasanshina, 2007).

\subsection{Implementation: best/good/bad practices}

Most of the developed countries that have already implemented tele-monitoring have had satisfactory results, but in developing countries such as Kosovo, the implementation of telemonitoring has faced various barriers, even though strategies have aimed implementation for many years back, implementation always failed or at least could not achieve satisfactory results (Peterson et al., 2016).

The evaluation of tele-monitoring projects/implementation/ initiatives has created the need for continuing the development of electronic health services. In most tele-monitoring projects has been evidenced the successful integration of tele-monitoring depends not only on the technical components but also on how and why the services are used. Assessments bring to light the successes and barriers of services that are important to patients, health workers, investors and other stakeholders (WHO, 2016b). In the next few paragraphs we collected and summarized a few experiences earned through eHealth and tele-monitoring systems implementation.

(Smaradottir, Gerdes, Martinez, \& Fensli, 2015) gave an account of the United4Health project (UK). It was launched, in 2008 and tested on 6191 patients with chronic illness. The project was initially focused on three different tele-monitoring services, including teleconferencing, monitoring and health training via text messaging (SMS) and online health monitoring and support. In the UK NHS digital services had been extended to more modules to attract health professionals and patients as well. Implementation takes time, in case of some modules up to 12 years, or is still under development due to the ongoing technology evaluation (Grosios et al., 2010).

Estonia in 2008 launched the E-tervis project (Lai et al.2013) whereby it has created the opportunity for patients, including foreign nationals, to see EHR using their ID card in combination with a mobile phone ID. This template is interactive and web-based, the patients can see their own electronic medical records, and see which of the healthcare staff has visited their profile and status. The Ehealth project allows its citizens to reserve an appointment for 
medical examinations, to receive notifications from doctors or infertility and care providers. This platform also provides tele-monitoring services, vitro health checks, and many others.

In Denmark, the Patient@home project was launched, focusing mostly on patient rehabilitation, which offers up to 30 services ranging from home treatments to data systems optimization. This system enables Danish patients to reduce the length of their stay in hospitals, as well as reduced admissions to Danish hospitals as a result of tele-monitoring. The disadvantage in the Patient@home electronic platform is the separation of health professionals, as well as non-compliance or ignoring tele-monitoring from some profiles of health professional roles. One of the main problems of this project is that nurses did not have the authority to make decisions without the presence of doctors, and this reduced the confidence of the patients in this project (Stuart \& Weinrich, 2001).

In Hungary, PISCES (EUREKA) project tested monitoring of patients with cardiovascular disease, as well as their response to specific physical activity. The platform was developed for smartphones and used wearable sensors. In the project, they used patient samples at different ages. Finally, the project turned out to be successful, with some recommendations especially in adapting equipment to patients (Kö, Gábor and Szabó, 2015).

In Israel, one of the long-lasting projects that succeeded in implementation is the project called CAMONI. The platform starts with a launcher and is developed as a web-based virtual communication, which through modalities include blocks, forms and private chat rooms. The platform is supported by 16 different communities with various chronic diseases, among them diabetes, cancer and depression. Individual consultancy between patients and medical professionals is also an option (WHO/EU, 2016)

In India, Aerotel project had a positive impact on the life of the patients. Aerotel operates in more than 40 cities and is the main telemedicine project in India. This project focuses on cardiovascular diseases, remote monitoring ECG of patients, and recording heart attack events (WHO/EU, 2016).

In Serbia, VisMedic Platform is similar to MojTermin a national eHealth project. A successful startup operates the tele-monitoring platform, it allows patients to use the video 
link to consult with their doctors, asking information, advice or second thought (Velinov et al., 2015).

Successes and failures are always going with any project. Most of the projects that have stagnated in implementation have to cope with the lack of legislation, the lack of incompatibility between different technologies and standards. One of the reasons of the telemonitoring projects' failure is the inability to adapt the eHealth system to patients and healthcare staff, and to other stakeholders because of the long lead time of learning how the system is becoming part of the patient's daily life (WHO, 2012).

The failure of tele-monitoring projects comes as a result of the non-standardization of the systems recommended by the WHO, where most countries disregard WHO standards in the absence of funds, trying only to adhere to their national strategies, which then falls facing the global objectives of the WHO.

Based on the experiences of most of the above-reviewed projects and within the literature, the main challenges related to interoperability between equipment, protocols and other major technological actors. The privacy and data security across machines and systems, such as NHS in the UK, Medicare, LifeGuard, Health Gear, CodeBlue or AlarmNet in the US. This is also a challenge for the World Health Organization (WHO), which is helping countries through the development of strategies by various experts, as well as by the experiences and best practices of developing countries that have already implemented tele-monitoring solutions and are on the right track for the functioning of a genuine system. According to research done in developing countries, one of the key problems of tele-monitoring failure in these countries, as noted above, stay in the planning phase of the strategies, especially the loosely defined steps that need to be followed during the implementation. Due to the inadequate strategies and their non-compliance with the WHO's objectives has led to stagnation or even failure of projects during the implementation phase. In Kosovo, two projects failed, one funded by the Luxembourg government and the other funded by the state of Kosovo, where the latter is based on the current Strategy 2012-2020. The testing phase of the latter mentioned project was in 2016, but the trial has not yet started in any hospital, and there is no draft version of the first implementation of this project, although the planned 
budget for this project has been already spent prematurely. The main indicators and barriers in the implementation of tele-monitoring according to the are divided according to lack of human capacity, infrastructure, finance, productivity, as well as policies are missing. (World Health Organization, 2016)

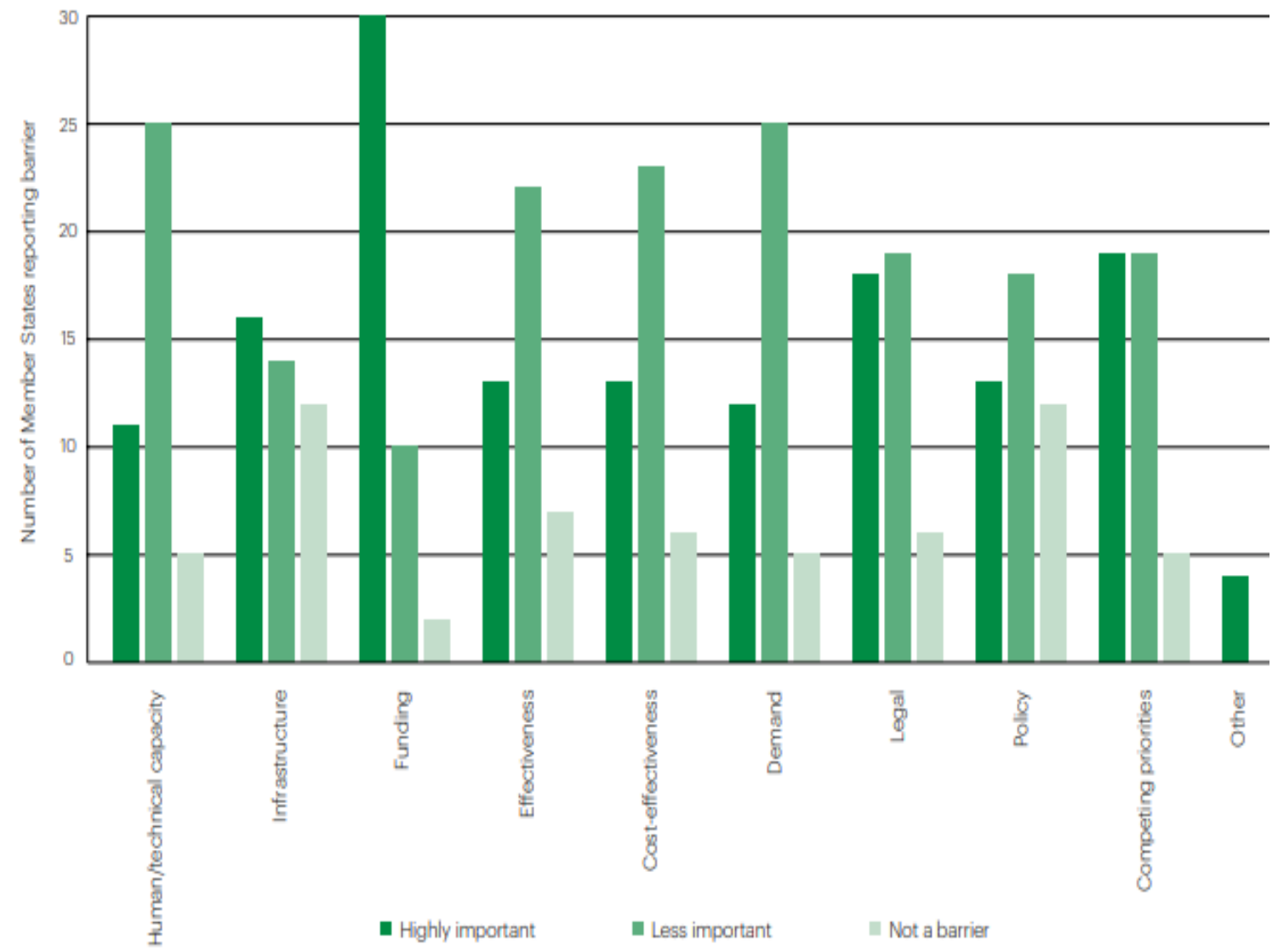

Figure 11. The main indicators and barriers in the implementation of tele monitoring (WHO/EU, 2016)

\subsection{Summary}

From the perspectives of improving performance outcomes in the health system also indicators are important, as they represent important aspects of the health system performance as well as they are the basis of quality assessment of health services. The purpose of these indicators is to measure progress in achieving results and assessing their impact. As seen on the WHO basis, the main indicators are divided into three main areas: socially, technologically, and financially. All of the indicators mentioned above are important, especially the health status indicator (health status), where research shows that 
both the International and the Balkan aspects of population mortality is higher, due to the lack of an efficient health system for treating the population. Therefore, this indicator drives us as researchers to contribute to the improvement of the health services, especially in developing countries, as the lack of digitalization of the health system results in inaccurate data on the health status of the population and all healthcare processes. The financial aspect is seen as a key indicator in monitoring the health system. As it can be seen in the data above, the reason for all states, failing to provide effective monitoring of the health system is that they spend their entire budget on medical treatments. Whereas, on the other hand, the implementation and use of ICT in health care seems to have borne the first fruit towards a quality service. Tele-monitoring is considered a promising and effective way to provide more integrated health services in the patient home. Tele-monitoring can be useful for citizens (patients) and care professionals, but also for health organizations and public authorities. The benefits of tele-monitoring, as mentioned in the literature, are in many respects: costeffectiveness, better use of medical personnel, easier access to health services. Based on the literature review, key indicators and barriers to tele-monitoring are divided depending on the lack of human capacity, infrastructure, finances, productivity and policies are lacking. 


\section{Problem Definitions - Research Questions}

\subsection{What Do We Want?}

Health information is important. The progress of healthcare delivery relies on making good decisions and the appropriate strategy. Good information enables decision-making either to provide better treatment or to orient health institution in the most efficient way or to ensure that the population of Kosovo is being provided with the proper health care service. The current Kosovo system and strategy for the past seven years has demonstrated the failure of functioning of the healthcare system and low performance in providing timely, effective, efficient and safe patient care. First and foremost, a static application was developed only to preserve the patients' historical data, without a function or even a goal that in the future this application would have the potential to be developed and become a system that includes all key stakeholders in healthcare system.

Based on the current situation illustrated above, I intend this research's output to be used for a Strategy Proposal to design and implement a healthcare management system. The strategy

focuses on ICT as a basis enhancing the opportunities of healthcare in Kosovo. The cornerstone is monitoring of the most common diseases in the country, which may have a negative impact on patients' lives. Using ICT in the healthcare management system, Kosovo may have better fiscal management in healthcare, more effective prevention and improvement in mortality rate, which according to the reports mentioned above currently is too high.

Improving the system in Kosovo can be can be carried out by understanding the national requirements and problems as well as through the use of engineering, management and different IT design and development solutions, among many others TOGAF, which in the world of information systems have helped many international strategies to succeed in the implementation of health information systems.

Therefore, I aim to elaborate and deeply scrutinize in this dissertation a strategy regarding the implementation of a functional health information system that responds to the current health situation and requirements in Kosovo, which is in coordination with the objectives of 
WHO for developing countries. Through existing technological capacities, I think that the above-mentioned problems can be solved by at least $30 \%$ in the next 5 years.

The main vision in this thesis proposal is to improve the health of patients as well as to raise awareness of patients to self-manage their lifestyle and enable candid health. One needs to design his or her own telehealth program based on the desired organizational goals, population, competencies, and partnerships, consequently, 30\% improvement means the following:

- Reduction of the overall morbidity and mortality rates of the population;

- Improving resource management and quality of services;

- Functionalizing, re-organizing and supplementing the existing infrastructure of the healthcare system and medical devices, in accordance with European standards.

In order to realize the vision, I have set some of the objectives below to be pursued in order to justify the requirements, which were highlighted above, concerned with improving the health system and health services in Kosovo. The purpose of this proposal from a technical point of view that strategy should be based on the current situation and problem definition. The distinction between the main objectives and specific purposes leads to far more multifaceted and comprehensive outcomes. Maylor and Blackmon (MacLean, 2013) suggest that specific objectives should meet the criteria of the well-known SMART test, which are as follows:

- Specific - What precisely do you hope to achieve from undertaking the research?

- Measurable - What measures will you use to determine whether you have achieved your objectives?

- Achievable - Are the targets you have set for yourself achievable given all the possible constraints?

- Realistic - Based on your opportunities would it be possible to meet the tasks and carry out this research on time

- Timely - Will you have time to fulfill all your objectives within your expected deadline? 
The main purpose is:

Proposing a strategy of an eHealth system that can respond to the demands and offer a comprehensive tool to help people in self-management of their health status through existing devices and infrastructure.

Whereas, the specific objectives are:

- Through existing technology (web and cloud platforms, WAN and LAN network infrastructure, sensors, mobile devices) and the proposed strategic eHealth information system to monitor and reduce the number of patients' visits to doctor and to increase patient awareness for self-management.

- Applications should be deployed on mobile devices with friendly user interface and to be applied by the patients on remote locations

- The system should be scalable and secure from communication and patient data management point of view, safety of wearable devices and patients should be guaranteed.

- The recommended system should reduce expenses for patients, hospitals and all other stakeholders involved in healthcare.

\subsection{What Can We Do? Architecture Principles}

In the thesis, I propose a high-level architecture of the Kosovo eHealth System, as well as in this document will I will draft the important cornerstones of implementation of the eHealth information system.

Architecture development covers definition of needed resources, taking into account the organizational barriers, implementation schedule, and the phases to be followed from the baseline to the target architecture. Implementation aspect covers ICT equipment and services to be procured.

The architecture will be based on the principles as follows:

- $\quad$ Equality and non-discrimination in the provision of services and employment 
- $\quad$ Protect the rights of the stakeholders, with a special focus on vulnerable groups

- $\quad$ Acceptance of diversity (cultural, social and professional)

- Accessibility (in geographical and/or economic aspects)

- Cost-effectiveness and reliability

- Quality of care

- Scalability

The proposed architecture will reflect to the existing infrastructure with the aim of approaching the one, specified in the architecture vision. The key success factor is the early establishment of an eHealth ecosystem consisting of several varieties of devices, applications and support systems. Another key success factor is taking into consideration the security risk, avoiding misuse of data. Giving priority to the high level of standardization the architecture fits in the future and foreseeable technological innovations. Beside of ICT infrastructure issues, also the process definitions and/or improvements must be addressed according to:

- $\quad$ Performers (health professionals, administrators, associates and policy-makers, users and service buyers)

- $\quad$ Procedures to be followed in different processes

- Input/output information (processing, transmission)

- Backend and frontend systems that facilitate the collection, analysis, storage and dissemination of data

Process definition will enable the architect to:

- Clarify the concept of the information system

- $\quad$ Define roles and responsibilities

- Identify the baseline architecture

- Develop a phased approach of creation eHealth information system complying with international standards. 


\subsection{Research Questions}

Based on the current situation and the above set goals I have addressed some research questions directly related to the objectives pursued in this research.

1. Will the proposed architecture (and how) reduce the level of progressity of healthcare in terms of resource usage and volume of patients?

2. Will the proposed architecture improve (and how) the collaboration among the various actors?

3. Will the proposed eHealth system (and how) scalable and modularized enough to address the different technical and cultural readiness of various users group? 
PART TWO 


\section{7. e-Health Enterprise Architecture}

\subsection{Why Enterprise Architecture Approach?}

Enterprise architecture is a complex systems development approach. The complexity comes from various factors. First of all this approach incorporates the business objectives, information systems design principles and methods, data management, technological platforms, implementation and deployment, with the stakeholders' analysis and requirement management, and the organizational aspects. In short, who does what, why, when and where, in the granulation levels scope, strategy, logical, physical design, implementation and change management? We need to emphasize three very important principles that are the cornerstones of the approach: architecture (building blocks, artefacts) repository, re-usability, standardization (interoperability).

In this chapter, the focus will be given mainly to the application, data and technology architecture, since the business architecture, related elements were verbosely discussed in the previous chapters, the implementation and deployment related issues will be discussed in the following chapter.

For us it is very important to put the emphasis on the evolvement of different architectures and their interactions in the ecosystem. Interoperability feature of the architecture is the basis for the proposed strategy providing undeniable proofs that ICT has the potential to properly address customer requests. From an architecture design point of view the information system architecture bears decisive role, especially in the context of different types of interoperability. Interoperability requirements can be understood and interpreted with the help of typology of ICT backed decisions as prerequisites of coherent system design.

These prerequisites are outlined in what form the patient has access to the system, how safe are the approaches to the system, how data exchange channels are implemented between these information systems, or what communications technology, web-based platforms should be used, what type of databases are used and how they are organized. The architecture is based on several essential aspects to guarantee a sustainable system based on the abovementioned objectives and are divided as follows (Figure 12): 


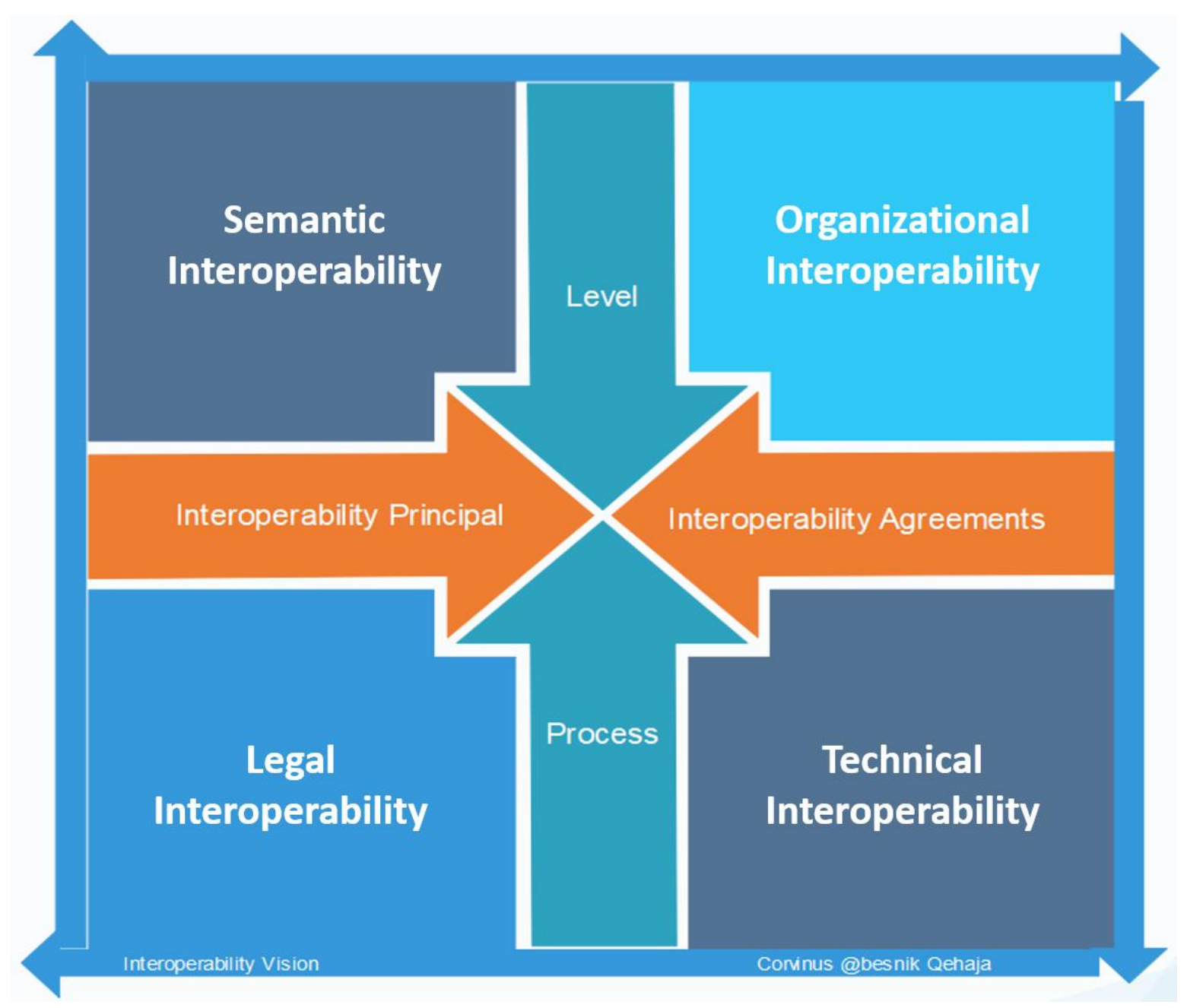

Figure 12. Interoperability in the architecture vision

The Figure 12 above shows the rules that must be followed, that each architecture should be scalable and provide a basis to meet the future demands of increasing the volume of data produced by health systems. Architecture will provide an approach that allows systems to share common functionality for integration. Architecture provides common data integration and management services as well as interaction with information that determines how information should be shared. Architecture primarily relies on international standards, that is a precondition providing secure environment, as a result of that confidential data will be kept safe. The architecture shall be capable of adapting to changes based on additional requirements for eHealth needs. The architecture should be consistent with widely accepted WHO standards that promote data, application, and technology sharing and collaboration. 
Technological interaction scrutinizes how ICT services such as communication networks and databases should be integrated and shared or at least related to each other's.

In terms of data the proposed architecture will be accounted for value assets that will help make the right decisions. The assumption relies on the extensive use of analytical platforms that are directly related to the separated but interdependent databases. A special focus will be given in the proposed architecture to security and data protection. Data security and protection should be approached on technological, application and regulatory levels. The proposed approach encourages the design and development of eHealth architecture that is consistent, reflects to the stakeholders' needs, uses best practices and pays due attention to current and future requirements, health needs.

\subsection{Current Situation of Healthcare Information Handling in Kosovo}

This research has been carried out by incorporating data reported at scientific level as well paying visits and making interviews with the respective interested parties. As a result of the collected data and feedback, there has been concluded that there is an urging need to undertake tremendous investments to improve the contact with patients.

The existing health system database called the 'DB003' is the most advanced part of the health information system, which is mainly paper-based, most of the patient data and their treatment are recorded in paper forms. These data time to time are collected by the respective institutions and recorded into an Access Database. Human resources data, patient information and schedule are mainly included into an Excel program. After services are performed access to data by medical staff or patients is limited to paper version. Investment in ICT infrastructure is a must, however reconfiguration of existing equipment's would result a significant improvement as they are already installed in the vast majority of the hospitals and used only a fraction of the performance they have.

Network infrastructure in most hospitals is mainly used for e-mail communication in the administrative offices, but wards are not connected to the Internet. The usual configuration consists of router, switch, PC, printer, scanner, camera. The software in most of the cases is Microsoft Office. Electronic communication in many cases provided with the help of IT professionals, who carry the messages on USB from one place to another. Based on reports 
and interviews, activity data, morbidity and mortality data in primary, secondary and tertiary care are increasingly being recorded in the main "Access-Master database". Nowadays Access databases in most regional hospitals are implemented locally, but the inclusion of data, relevance and validity are not satisfactory.

The data are reported with a delay of six months or more, not all health institutions report in a unique form, therefore similar data reports ending up with different results. The private health sector does not report at all. No hospital has access to the central database. In general, communication with the interested parties is carried out through telephone from the offices of doctors and managers of hospitals. Predominantly, the vast majority of hospitals possess technological equipment are not in a property of the institution, and is often maintained by third-party healthcare companies. Based on the IT contacts, the hospitals are provided with phone numbers from the state free Telecom for telephone calls when they have problems with the existing network and all the support is done through the phone. As far as communication of state network infrastructures is concerned it has given priority to most of hospitals, in order to have access to the fiber optic government network. Howerver, there no logical configuration that exploits the physical configuration to become a stable connection and infrastructure serving a common service across the country. In my opinion, a reorganization by utilizing the current resources would bring a good opportunity to implement the eHealth system.

Contrary to the existing situation we may come up to the conclusion that almost $92 \%$ of the Kosovo population uses a smartphone or a computer. In this perspective, it is worth pointing out that every second a citizen of Kosovo makes use of the internet in a smartphone or a personal computer. (KAS, 2017b) This indicator paves the path for the introduction of eHealth services. Patients may become an integral part of eHealth system by using their own personal devices. One of the problem stems from the difficulty of using digital medical services, but it will be the primary task to provide customized training for using the electronic healthcare system. 


\subsection{Stakeholder Analysis}

Based on the current situation and the functional requirements the proposed architecture is a solution that responds to current requirements and is in compliance with the standards in terms of technology and the compatibility recommended by WHO (WHO/ITU, 2012). Undoubtedly, by making use of this solution, we are pointing out where we are and how adequate existing technology solutions would have improved the current health system based on the best practices of the systems implemented so far. In my opinion, the proposed system architecture and the respective solution will be unavoidable. Since the beginning of this decade there has been argued that the current system will face major challenges regarding the upcoming technological changes and therefore my proposed architecture, would ease this transition and make it easier for developers to adopt technological trends and changes to the newly implemented healthcare system.

Before starting to propose the develop architectural layers I have tried to tackle and explain the aspects related to the role of stakeholders who are directly involved in processes related to the eHealth system. 


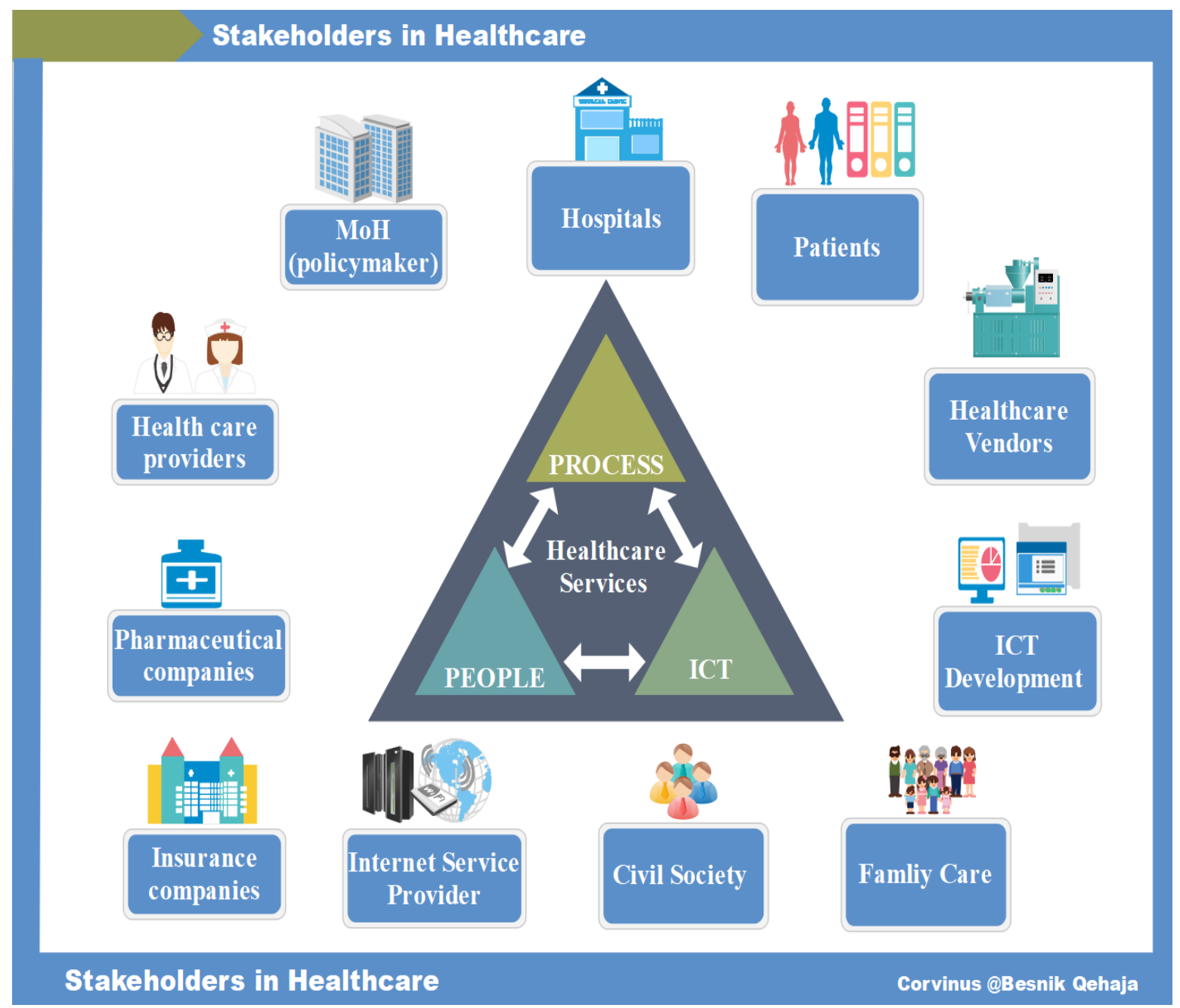

Figure 13. Stakeholder interaction in healthcare

Indeed, architecture it is important to investigate and understand the interested parties' requirements and their behavior in the healthcare system and returned them as functional user requirements as well as functional points of the system. During the literature review, we have specified each entity and also recommended a functional system using the three major architectures that represent the health information system.

In the Figure 13 above are given the parties whose interests are interdependent with healthcare. Communicating in healthcare can be: 
- Communication within a single healthcare unit.

- Communication between different medical units of a single healthcare institution / organization.

- Communication between the various medical and non-medical units of a single healthcare institution / organization.

- Communication between various medical institutions / organizations.

- Communication between medical institutions / organizations and other healthcare partners (e.g. pharmacies, laboratories, other services, institutes, etc.).

- Communication between healthcare institutions / organizations and non-medical partners in healthcare (e.g. insurance companies, funding organizations, public administration, etc.).

- Communication between national healthcare institutions / organizations and foreign healthcare institutions / organizations.

Each entity above has its own relevance with one-another and if we come up with an adequate technical solution that each entity and ICT are linked to, then the objective is thought to be realistic. Indeed if the method is that if we follow the steps like using engineering solutions where the application architecture receives information, architecture data that processes valuable information and choosing the infrastructure that supports digitization of processes and communication, we think that the information system will work based on the expectations we have set above.

Similarly, to architectures in other industries, healthcare architecture has been subject to a rapid change over time from that relatively stable industry in a dynamic industry. Health information systems have also evolved through several different technologies, so during my research in the literature and 14 years of experience as telecommunication engineer I have analyzed and planned a solution that for the next 10 years. The technology I propose in this architecture would be able serving for a longer period.

Initially before I argue the importance of architecture in the field of information system and health services in general, I go back to the general eHealth definition. This recommends any 
system that provides specific information and supports decision-making process at any level of an organization can be defined as a eHealth system. First and foremost, a health information system is a 'system' and just like any system that has an organized set of interacting components, this system can be grouped into two entities: the information process and the management structure. Through the information process, inputs are transferred to useful information for managing decision-making (outputs).

A representation and recommendation of the general eHealth architecture vision as seen below is recommended by my perspective of seeing the best and successful practices of the European countries that have been examined in the literature. I have proposed an architecture, which fulfills the requirements based on the current situation in Kosovo incorporating all stakeholders and their requests for exchange of information using ICT. 


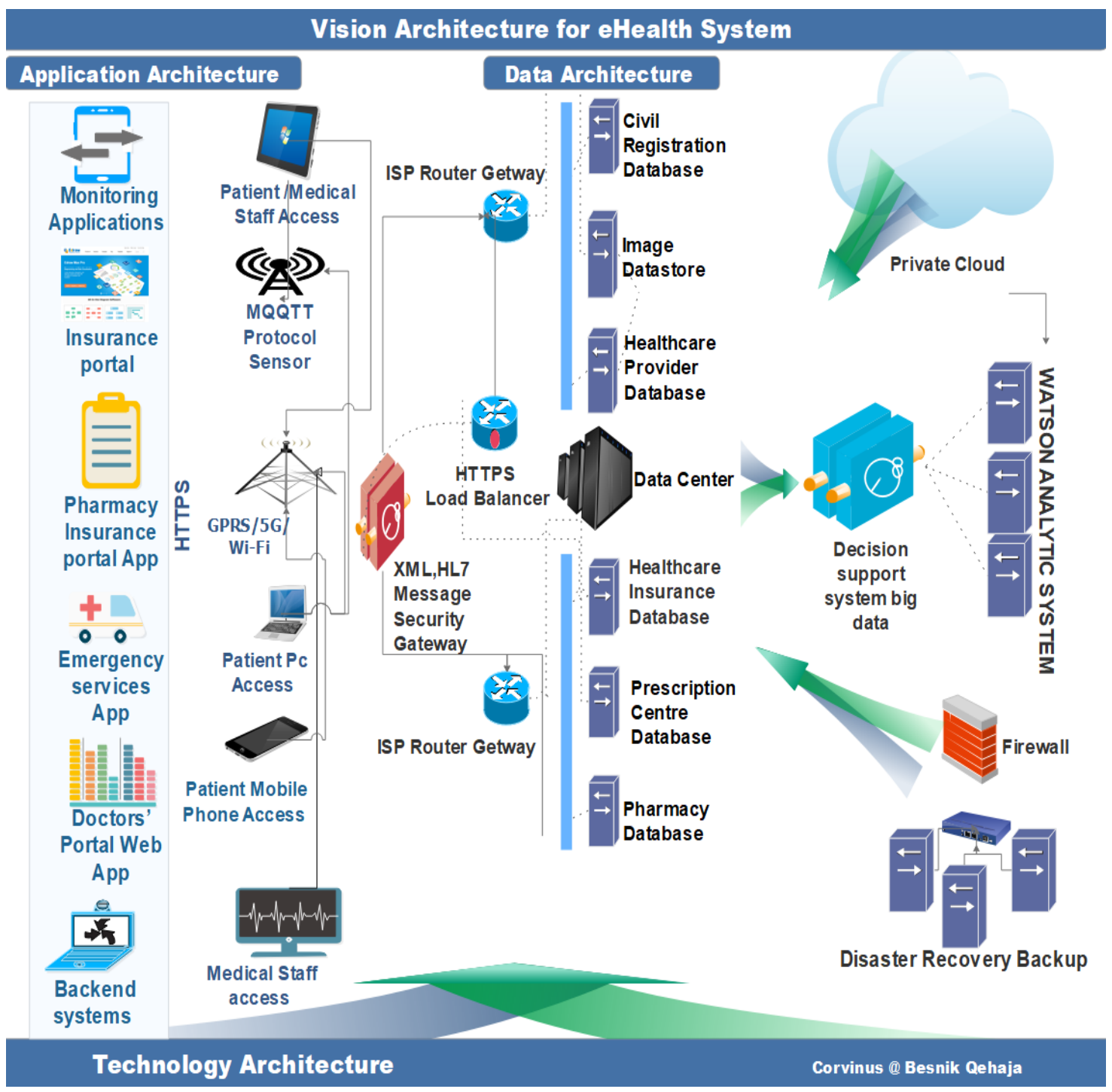

Figure 14. Architecture vision

As follows, I will subtly elaborate in detail the aspects of architecture in the information system at three levels in logical elements of communication at the level of application architecture, data architecture and technology architecture as well as the main functions of communication using visual images and justifying each function from an engineering aspect.

The architectural approach makes use of different types of communication using different technologies, as discussed below:

- Patient-related medical communication (anamnesis, diagnosis, therapy, monitoring, follow-up) 
- Patient-related non-medical communication - (patient account, resource usage, financial and administrative information)

- Non-patient-related medical communication - (mortality and morbidity statistics)

- Non-medical communication - e.g. materials, human capacities, materials in stock etc.

The information processing in the vision of the recommended architecture is split into several components of data and technology that will support the system as follows:

1. Collection of data through applications, sensors, smartwatch, mobile applications, web applications and standardized monitoring equipment.

2. Data transmission via e.g., Internet, computer devices, communication protocols like HL7, internal and external protocols in computer networks like 4G, 5G, WiMAX.

3. Data processing in XML data format, using specific databases for each entity present in eHealth system, personal data, and data generated from the aforementioned devices.

4. Analyzing data through systems using artificial intelligence, such as Big Data software, Watson Analytic, Siemens Health.

5. Presentation of information in forms of indicators for planning and management of health services. Analytical data can be filtered according to organizational processes such as medication procurement, hospital treatment, human resources etc.

6. Monitoring and evaluating the processes ensures that proper input mixing (inputs) for the sake of producing the right outputs at the right time. For example, the necessary information changes constantly with changing planning and management needs.

A health information system can generate the right information only if each of the components has been accurately structured. Working through the recommended architecture, the goal is to provide a system supporting the key system functional requirements; the most important ones are listed below:

- The system supports automatic translation of data codes used in medical records, communication. Code translation must include ICD-9-CM, ICD-10, CPT (4 and 5), SNOMED II and III. 
- The system should support the seamless communication among various applications regardless the paperless or hybrid systems.

- The system should be able managing micro services, enabling the data interchange among a variety of databases.

- The system integrates a variety of platforms and applications for data capture

\subsection{Application Architecture}

Application architecture consists of a variety of applications, including report generation, data capture and transfer from different devices, data visualization, access modules for medical and non-medical stakeholders. The high-level overview of application architecture follows the segmentation of stakeholder groups as it is shown on Figure 15.Figure 15. Application Architecture

\subsubsection{Functional requirements}

Following patients' movement and medical episodes is the basic principle behind of the application architecture. This principle demand to collect all relevant data historically and providing selective access to the properly authenticated and authorized personnel. Realization also demand the standardized data flow among the various applications and platforms. The detailed requirements explained in the following sections.

- Continuity of care: one or more healthcare providers provide services to a patient in a coordinated manner. This organizational principle focuses on time ties between various health services (focus on medical history).

- Joint care: two or more healthcare providers collaborate providing services to a particular patient for ongoing diagnosis and/or treatment.

- Careful care: focuses on the timely and adequate transfer of activities and information where the responsibility for providing healthcare has been fully or partially transferred from one healthcare provider to another (focus on shared medical intervention) 


\subsubsection{Target groups}

Monitoring can be specific according to the target groups. Services cover many areas of healthcare, including ECG monitoring, blood pressure, glucose, respiratory, external factors (humidity, wind, and temperature), drug taking rigorosity and physical activity through standardized sensors that can be directly linked through API to mobile application. The target groups fit to the following categories:

Patients with chronicle diseases are patients who do not need any acute care, but only regular checkups. They must keep themselves to the prescribed medical procedures and ensuring drug compliance as advised by the doctors and other medical staff is very important.

Patients with acute interventions are people who in the past have had an intervention, especially surgeries like arthroscopic or heart surgeries. This group of patients, usually spend a time between three to six months (as advised by the doctor), in rehabilitation centers. For this reason, they have to be monitored on a real time basis, so that the doctor can keep an eye on them.

Healthy people can also utilize the applications, which in the following years will be much more developed with additional functionalities, to keep track of their health, in order to prevent potential diseases. Fitness sensors, communities, tracking through smartwatches or any other mobile devices and many more technologies will be used to keep an eye on this target group as well. By monitoring this group gives the opportunity evaluating the collected data, the results will show their current health status. This solution allows healthcare providers to interact relatively continuously and without interruption and use predicative analysis for launching and improving preventive measures, including direct screening actions, or customized and personalized "warning" reports. 


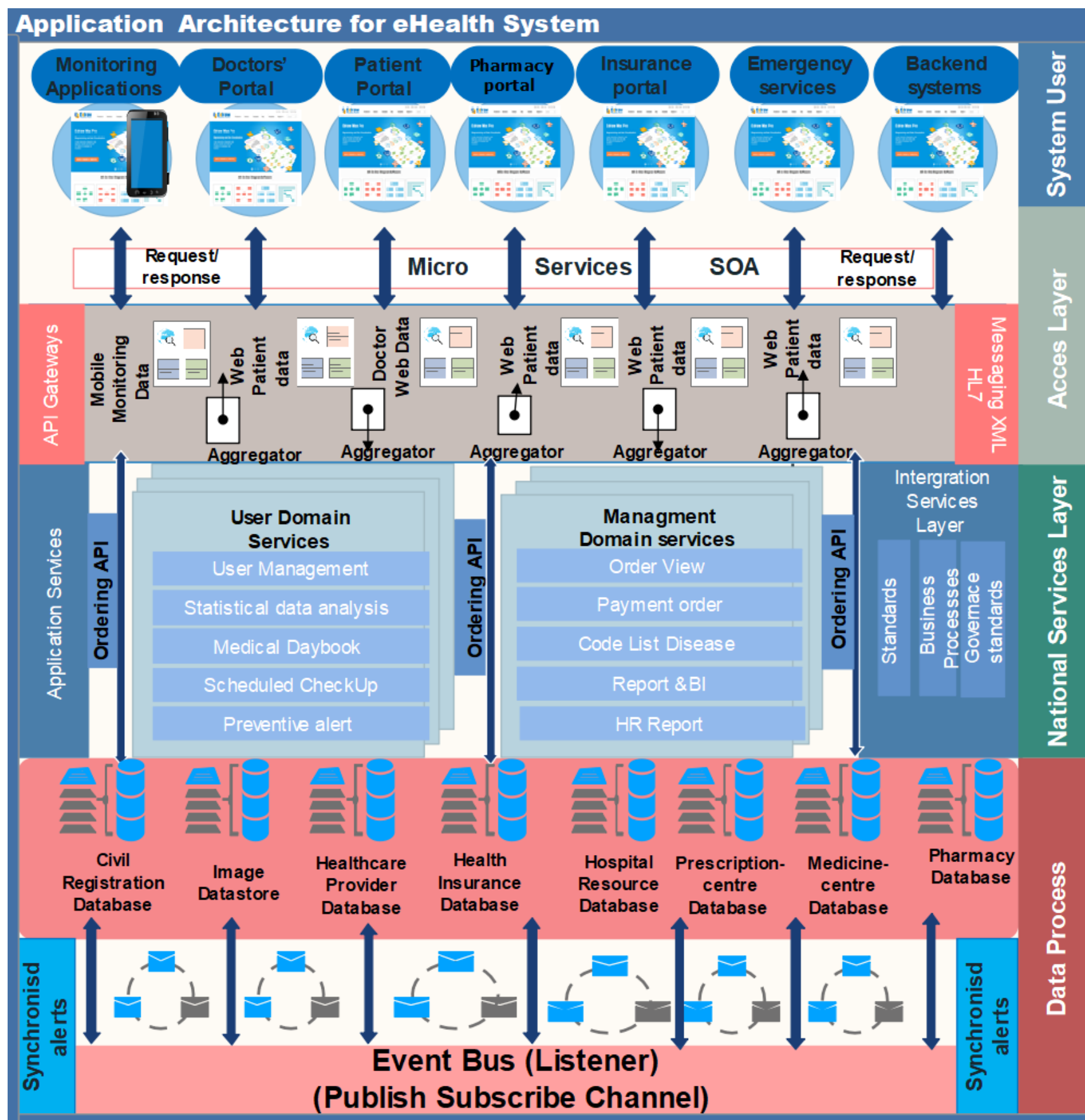

Application architecture

Corvinus @Besnik Qehaja

Figure 15. Application Architecture 


\subsubsection{Applications}

The eHealth application architecture contains those applications, which are relevant for providing, and managing healthcare services in the eHealth ecosystem. Below are some of the most important parts of the application architecture:

Patient Portal is an application through which patient can communicate with the service providers. After the patient enters the web portal with the credentials assigned to him/her and registered as a client at a specific healthcare provider then he/she can view a patient related information, e.g. regularly monitored disease, labor diagnosis results, medical recommendations, history of prescriptions, doctors availability, etc. The advanced web portals also incorporate chat-bots, that allow a user to discuss treatment, or administrative matters.

Doctors' Portal this is similar to the Patients' Portal, but data access and information services are more detailed and customized on a professional manner. Tele-monitoring module, which is specifically designed for the doctor, collects high volume of data, but doctors are interested only in those that are different from normal. Therefore, they use a dashboard, where only interesting patient data (the irregularities) show up, including but not limited to: the status of sensors monitoring the patient, prescriptions, current treatments, duration of cure etc. This portal is related to the healthcare providers' databases and from this portal the doctor can access those data of his patient, as well. It is noted, the access and use of patient data is under special and very strict regulation, this is explicitly controlled by EU regulations and specifically the GDPR. The strict authentication and authorization providing enough security and privacy will be detailed in the technology architecture section. 


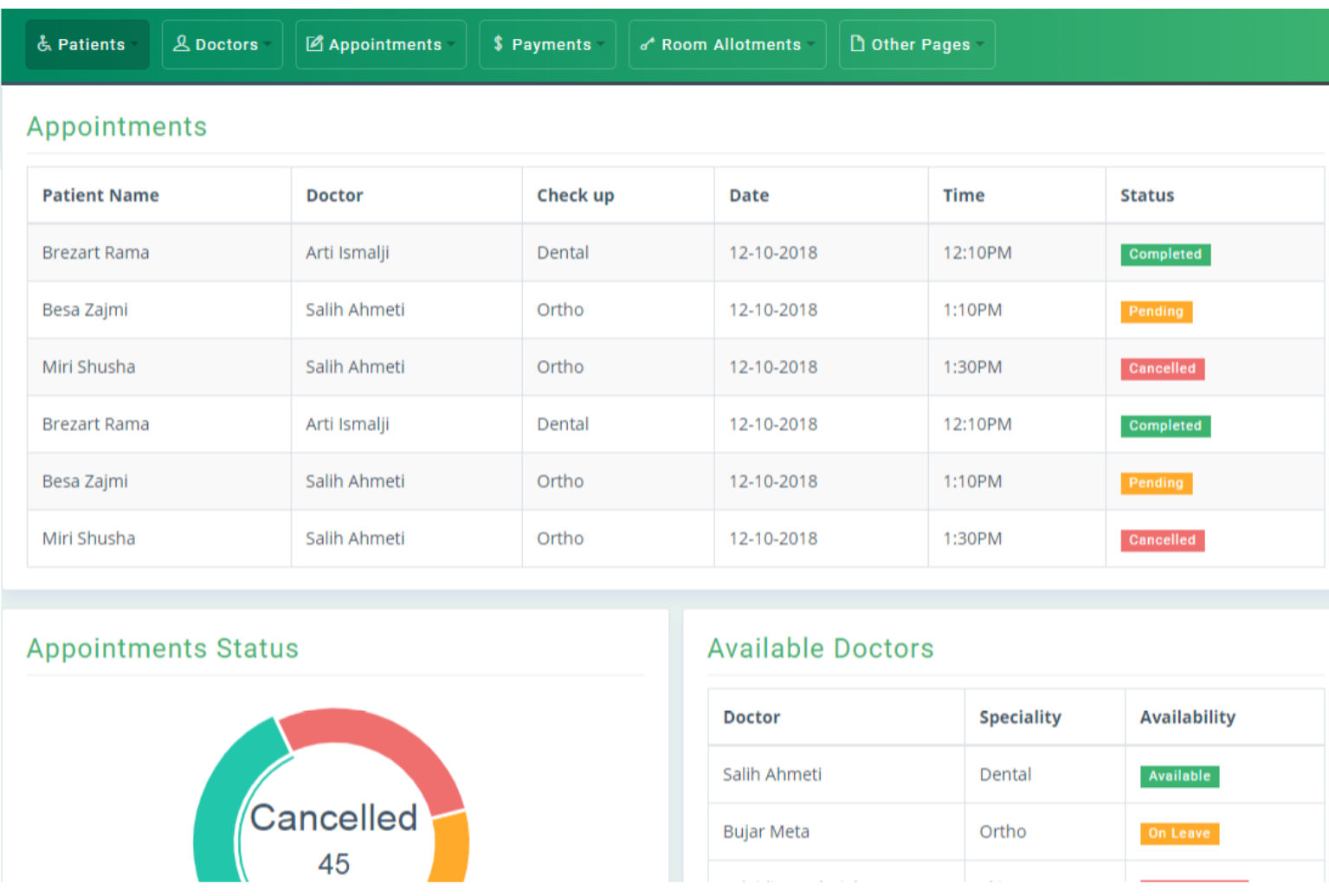

Figure 16. Appointments dashboard

Emergency services is an application designed to keep track of the status of the patients being in critical status, alerting medical staff in case of emergency. The scale of intervention is wide, from informing the doctor to providing help on site. A good example of interoperability and collaboration that architectural approach ensures, how the intervening emergency team get address, and absolutely needed medical information. The address of patient in emergency is automatically obtained from the database and given to the emergency response team, to locate the patient with the other urgent medical information.

Insurance portal This portal allows a patient to check its insurance status, the amount that the user has paid for the insurance, services the insurance covers, obtained services that insurance covered. This portal is connected with the Insurance database, from which the user can read its data. In a more advanced solution, partly as a result of the architectural approach, the insurance fee can be connected to the healthy life mode, and in return of screening or any preventive action, the insurance company or fund can give some discount, or on contrary, can punish the patient. 


\section{\& Patients - \& Doctors - $\square$ Appointments - \$ Payments * of Room Allotments - $\square$ Other Pages}

\section{Payments List}

\begin{tabular}{|c|c|c|c|c|c|c|}
\hline 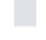 & Patient Name & Doctor Name & Service Name & Charges & Discount (\%) & Status \\
\hline 口 & Brezart Rama & Salih Ahmeti & X-ray & $\$ 500$ & 5 & completed \\
\hline [ & Genta Zeqiri & Salih Ahmeti & ECG & $\$ 120$ & 10 & completed \\
\hline 口 & Vlora Shala & Salih Ahmeti & Dental & $\$ 190$ & 8 & Pending \\
\hline a & Brezart Rama & Salih Ahmeti & X-ray & $\$ 500$ & 5 & cancelled \\
\hline 口 & Genta Zeqiri & Salih Ahmeti & ECG & $\$ 120$ & 10 & completed \\
\hline L & Vlora Shala & Salih Ahmeti & Dental & $\$ 190$ & 8 & Pending \\
\hline 口 & Brezart Rama & Salih Ahmeti & X-ray & $\$ 500$ & 5 & completed \\
\hline L & Genta Zeqiri & Salih Ahmeti & ECG & $\$ 120$ & 10 & completed \\
\hline L & Vlora Shala & Salih Ahmeti & Dental & $\$ 190$ & 8 & Pending \\
\hline L & Brezart Rama & Salih Ahmeti & X-ray & $\$ 500$ & 5 & cancelled \\
\hline
\end{tabular}

Figure 17. Payments list dashboard

Pharmacy portal historically allowed the patient to track purchases and insurance or social security funding of pharmaceutical products. Introducing e-receipt, medical institutions and pharmacies send and receive prescription data through the eHealth platforms, also the customer (the patient) will log in the system on the premises of the pharmacy, to get the prescribed medicaments. This way the total sales and funding can be controlled. An additional service what the pharmacy can provide to the doctor and to the patient is the drug interaction check, also the consumption tracking can be a good starting point of fighting against polypragmasy.

Monitoring applications aim to collect data from the patients and from the environment in which the patient lives. Hence, the main beneficiaries of monitoring services will be patients, while their relatives will be the secondary beneficiaries. Monitoring services can be 
implemented in many ways: fixed sensors, wearable sensors, web applications (e.g. weather information), smartphones, and dedicated medical devices (e.g. Holter). Through mobile applications patients can be monitored in-house or on the workplaces, or during sport or leisure activities.

The monitoring applications are connected to the eHealth backend systems, continuously transmitting data or in batch mode. Through configured API they would be linked to the recommended Watson Analytics system than medical staff could take precautionary measures in real time. This monitoring applications may be used monitoring specific groups of people who have a higher risk to become a patient, considering their profile. Examples of special groups: office workers who have static jobs that do not have activities within the day of the week; drivers who do not have activities but need to have preventive actions to keep them healthy to do their jobs. Monitoring should cover not only the very strict parameters, but the specifics of healthy lifestyle, including physical activity, nutrition, even some of the parameters showing mental status.

Backend systems will handle and manage databases, decision support actions, and statistical-epidemiological reports. The data processed "out of the monitoring time slots" provide valuable raw material for preparing and/or fine-tuning medical policy, especially in direction of prevention. A data warehouse type application will give many opportunities for analysis, drill-down, or other analytical approach (Figure 18.). Hence the real-time monitoring of patients with acute or chronicle diseases, easily leads to create an eHealth ecosystem which ends up in a significant and expected cost saving. 


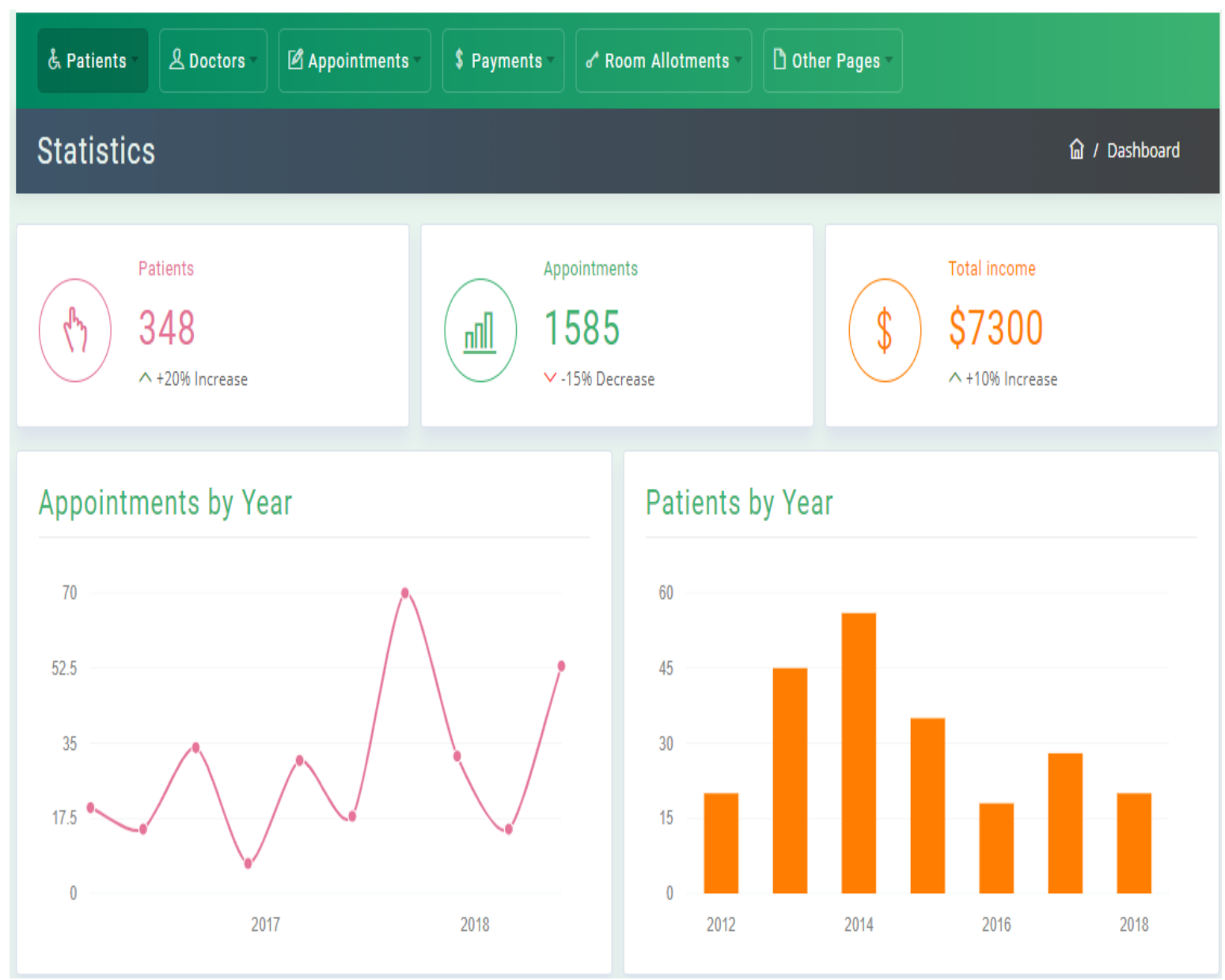

Figure 18. Analytical dashboard 


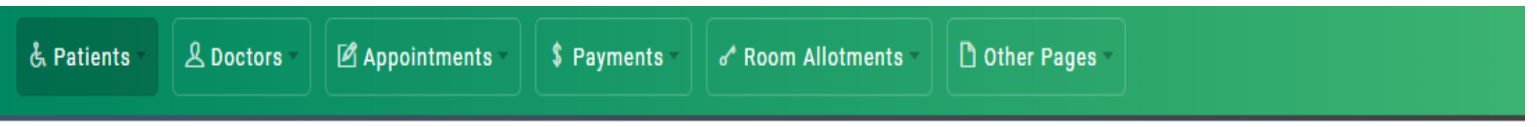

Allotments List

\begin{tabular}{|c|c|c|c|c|c|c|c|}
\hline & Room & Room Type & Patient Name & Allotment Date & Discharge Date & Doctor Name & Status \\
\hline 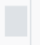 & 10 & ICu & Brezart Rama & $12-11-2018$ & $16-11-2018$ & Dr Salih Ahmeti & Available \\
\hline 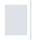 & 4 & AC Room & Blerta Nitaj & $10-11-2018$ & - & Dr Salih Ahmeti & Not Discharged \\
\hline [ & 2 & AC Room & - & - & - & - & Not Alloted \\
\hline 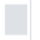 & 3 & General & Miri Shusha & $10-11-2018$ & $12-11-2018$ & Dr Shpresa Rama & Available \\
\hline te & 10 & Icu & Brezart Rama & $12-11-2018$ & $16-11-2018$ & Dr Salih Ahmeti & Available \\
\hline 1 & 4 & AC Room & Blerta Nitaj & $10-11-2018$ & - & Dr Salih Ahmeti & Not Discharged \\
\hline 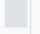 & 2 & AC Room & - & - & - & - & Not Alloted \\
\hline 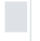 & 3 & General & Miri Shusha & $10-11-2018$ & $12-11-2018$ & Dr Shpresa Rama & Available \\
\hline . & 10 & Icu & Brezart Rama & $12-11-2018$ & $16-11-2018$ & Dr Salih Ahmeti & Available \\
\hline & 4 & AC Room & Blerta Nitaj & $10-11-2018$ & - & Dr Salih Ahmeti & Not Discharged \\
\hline
\end{tabular}

Figure 19. Allotments list Dashboard

\subsection{Data Architecture}

The interoperability of the eHealth system operates only when the data architecture allows transmitting information between two or more systems allowing one another to process the information in an independent manner and with a single standard. I have tried to compile flow of data where stakeholders in this architecture have unique data from different databases.

When using DBMS, the information system can be adapted according to information gathering requirements. Additional categories of data can be added to the database without jeopardizing the existing system. Database servers are located on dedicated computers. 
Generally speaking the whole range of these servers are multi-processor computers, with abundant memory and independent disks in the form of Raid 0 to raid 10. DBMSs are connected to one or more servers through a high speed channel, also used in the transaction of high volumes. The DBMS is located at the heart of most database applications.

Below is the data architecture for eHealth and components are divided as follows:

- Civil Registration Database

- Image Data store

- Healthcare Provider Database

- Health Insurance Database

- Hospital Resource Database

- Prescription-center Database

- Pharmacy Database

- Medicine-center Database 


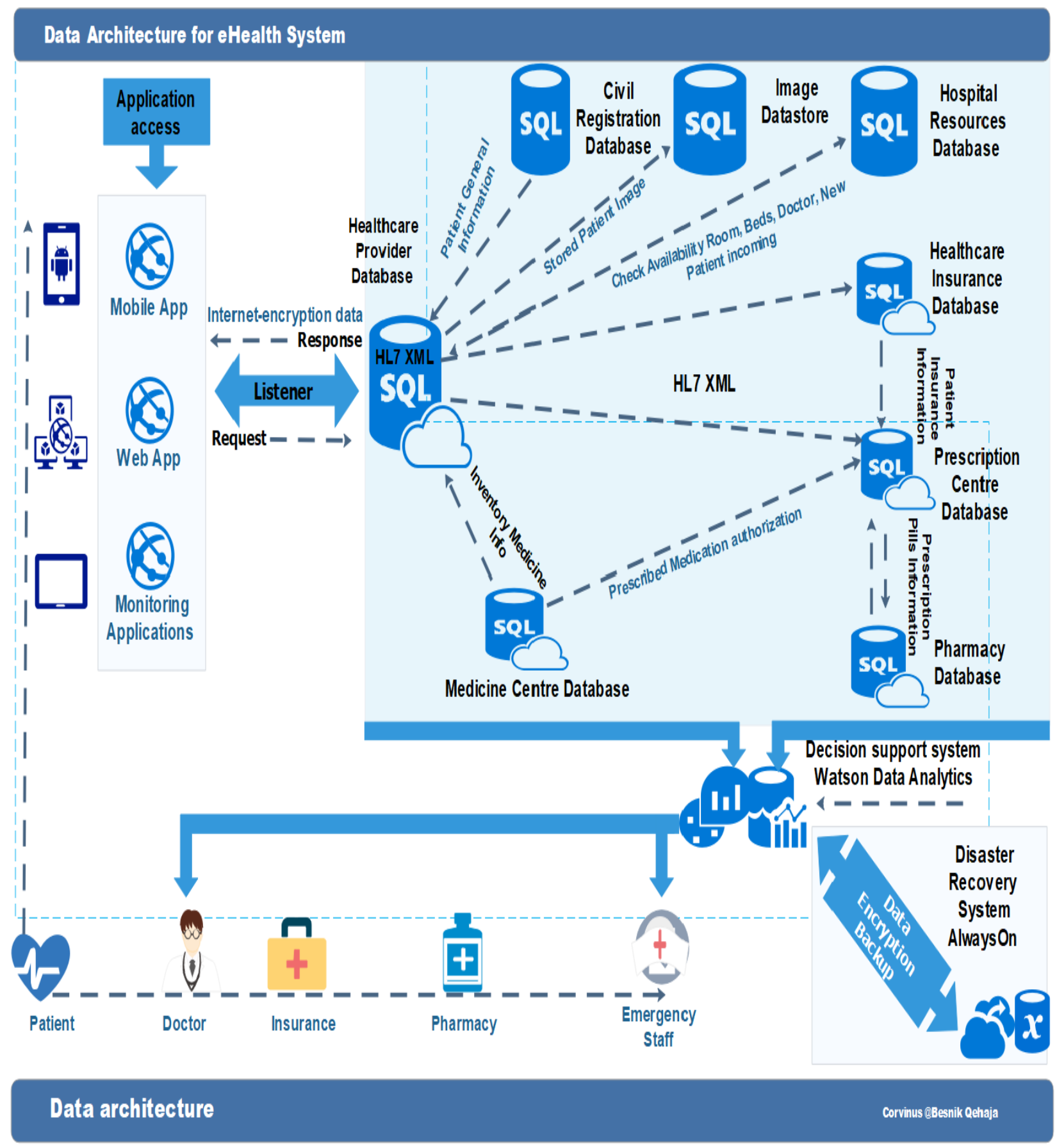

Figure 20. Data Architecture

Figure 20 describes the data and information that flows among the databases in the eHealth system. 
The government runs the Civil Registration Database. This database contains matricula and address data of every citizen, it is used by the healthcare service providers to record patients automatically with the correct and accredited information. From this database the healthcare providers are informed about newly born citizens.

The Healthcare Provider Database operated and maintained by the healthcare provider, stores all the healthcare data that was generated under the medical services. The X-rays, US, CT and other images are sent to the Image Data store.

The Image Data store is a dataset, which stores all images related to a patient.

Hospital Resources Database contains the data, which is used to track the available rooms, the available beds on those rooms, the actual number of patients and the number of new patients coming in. The healthcare provider always communicates with this database in order to check availability, and in the same way, using the database enables the healthcare provider to act, if the number of available rooms or available beds is decreasing.

Healthcare Insurance Database stores all the patients' data that pay for their health insurance products. The healthcare provider always checks if a patient is insured by a partner insurance company before treatment or if the patient pays himself. If the patients is insured, than the healthcare provider sends the patient information and the bill for the treatments to the healthcare insurance company. The healthcare insurance company also sends patient insurance information to the prescription-center to prove that the patient is indeed insured by them.

The Prescription Centre Database stores all prescriptions that were prescribed by the doctors of the healthcare provider. The healthcare provider stores all the diagnosis, treatment related data plus the prescribed medicaments for each patient separately and sends the prescriptions regularly to the prescription center. The prescription center transfer the prescriptions to the pharmacy database to check availability and in case of out of stock suggest replacement.

The Pharmacy Database stores information about all the currently available drugs in store. It also stores the OTC sales and the ones for which healthcare insurance paid in order to send the bill to the healthcare insurance company. The pharmacy database receives prescription 
from the prescription center; this is how they check the current inventory. They also provide information on stocks to the prescription center.

Medicine Centre Database has a very close relationship with the pharmacy, owing to the fact that it controls all the inventory that is currently available in pharmacies and makes connections between pharmacies to know which one is in possession of which drug, so that the exact drug can be found for patients in need. This information is fed to the healthcare provider so that they know, which drug to be prescribed and which not in case of nonavailability. Confirmation about prescribed drugs is sent to the prescription center to inform them on the prescribed drug's status. 


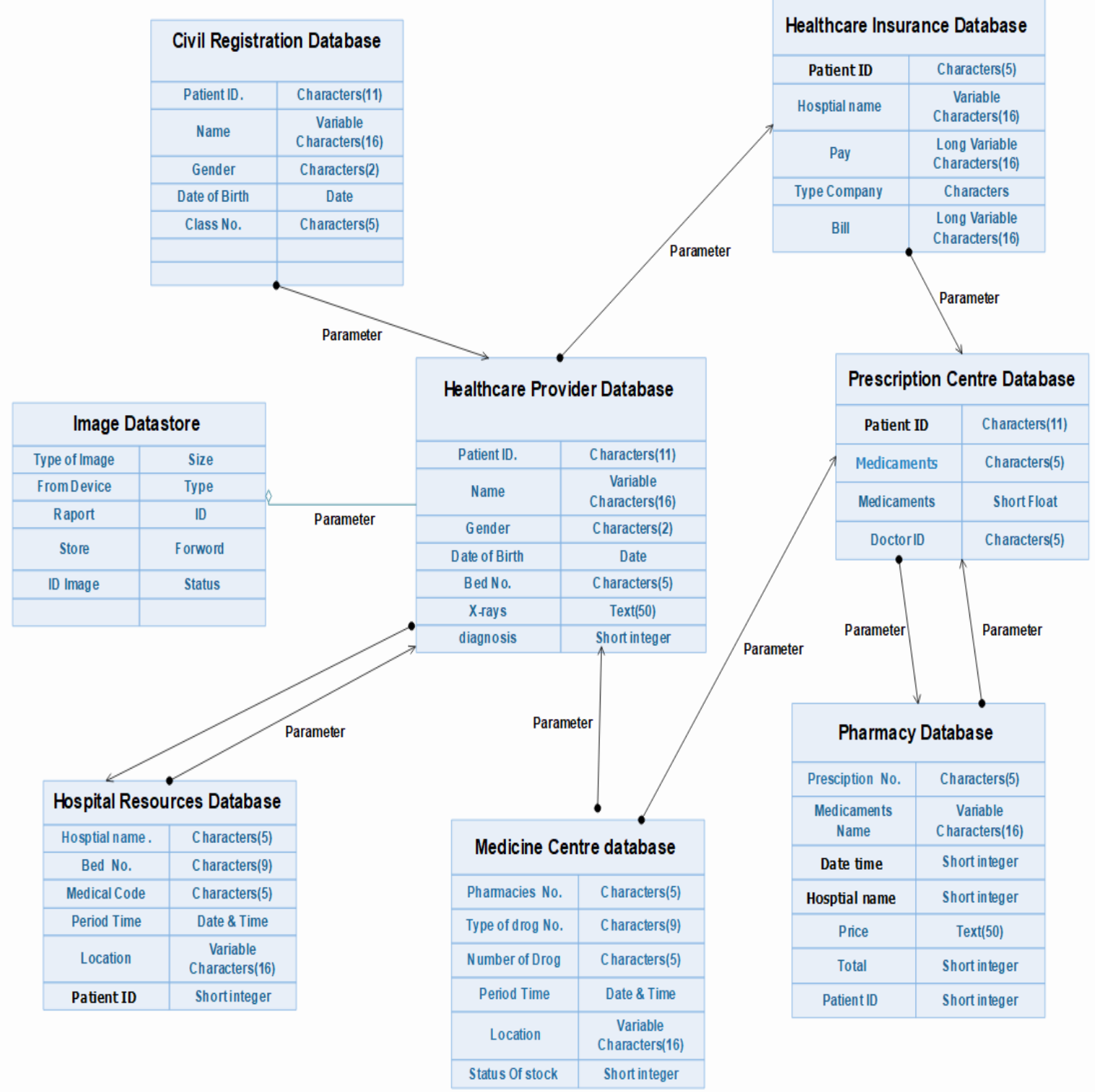

Figure 21. Database relation structure

\subsubsection{Data analytics in the eHealth system}

The ultimate goal of the eHealth system is to have better information on evidence-based decision-making. Different stakeholders in the health sector have a different focus and will use the eHealth system for various purposes. The proposed solution below relates to the data that are collected, and are distributed among various databases and processed by the 
Statistical System through APIs to generate useful information. This makes the data ready to be analyzed and sent to AI services to provide quickly health reports. The proposed technology is IBM Watson Health designed to address some of the world's most important health challenges through data, analytics, and AI (artificial intelligence), doing this via capturing, processing and displaying valuable data for stakeholders and providing information for future forecasts (Figure 22).

\section{Data Architecture for eHealth System}

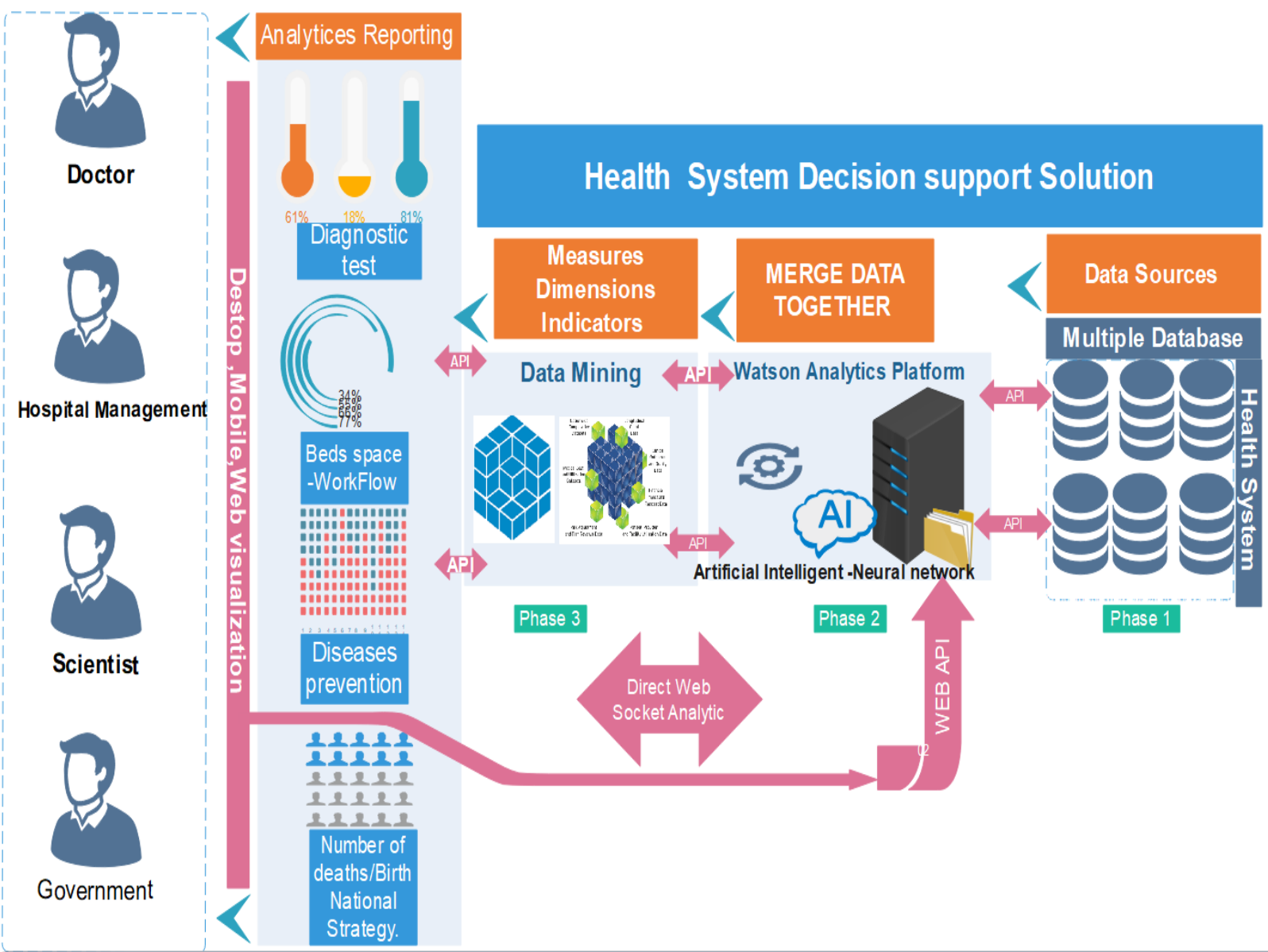

Figure 22. Decision Support System

Through the proposed statistical system, patients will decide where they can handle best their current problems. Health professionals will make decisions about the best ways of treating patients. Administrators and politicians will use the statistical decision-making system on 
how to prevent illness or to ensure high levels of quality, productivity and effectiveness of human and managerial resources within hospitals such as: planning for hospital places, flow of work and processes, shortening of expectations. For scientists the system provides input for analyzing serious diseases and for other scientific purposes. Government make use of information on mortality, birth and future health strategy plans to improve the quality of health.

Adaptation of the decision support system is driven by the number of sources of healthcare information such as EMRs, laboratory systems, diagnostic or monitoring instruments, insurance / billing requirements, pharmacies, human resources and vendor/supplier information, and real-time detection systems. The availability of as many healthcare data as possible is crucial, as stakeholders create more and more indicators for decision-making. This is true on the other way around. If data is available, decision makers can create more granulated indicator set. Watson also supports data mining and exhibits the results in a statistical form. Watson also allows us to create API, which receives data from Web applications and generates reports.

\subsubsection{Disaster recovery - AllwaysOn}

In the data architecture the ecosystem is mirrored, where many data are stored in different databases and for eHealth system it is vital to maintain and secure the data. Therefore, in the data architecture, I have proposed a solution for data recovery in case of disaster, malicious threats or any other legal problems. Below a planning and data management architecture is illustrated (Figure 23). 


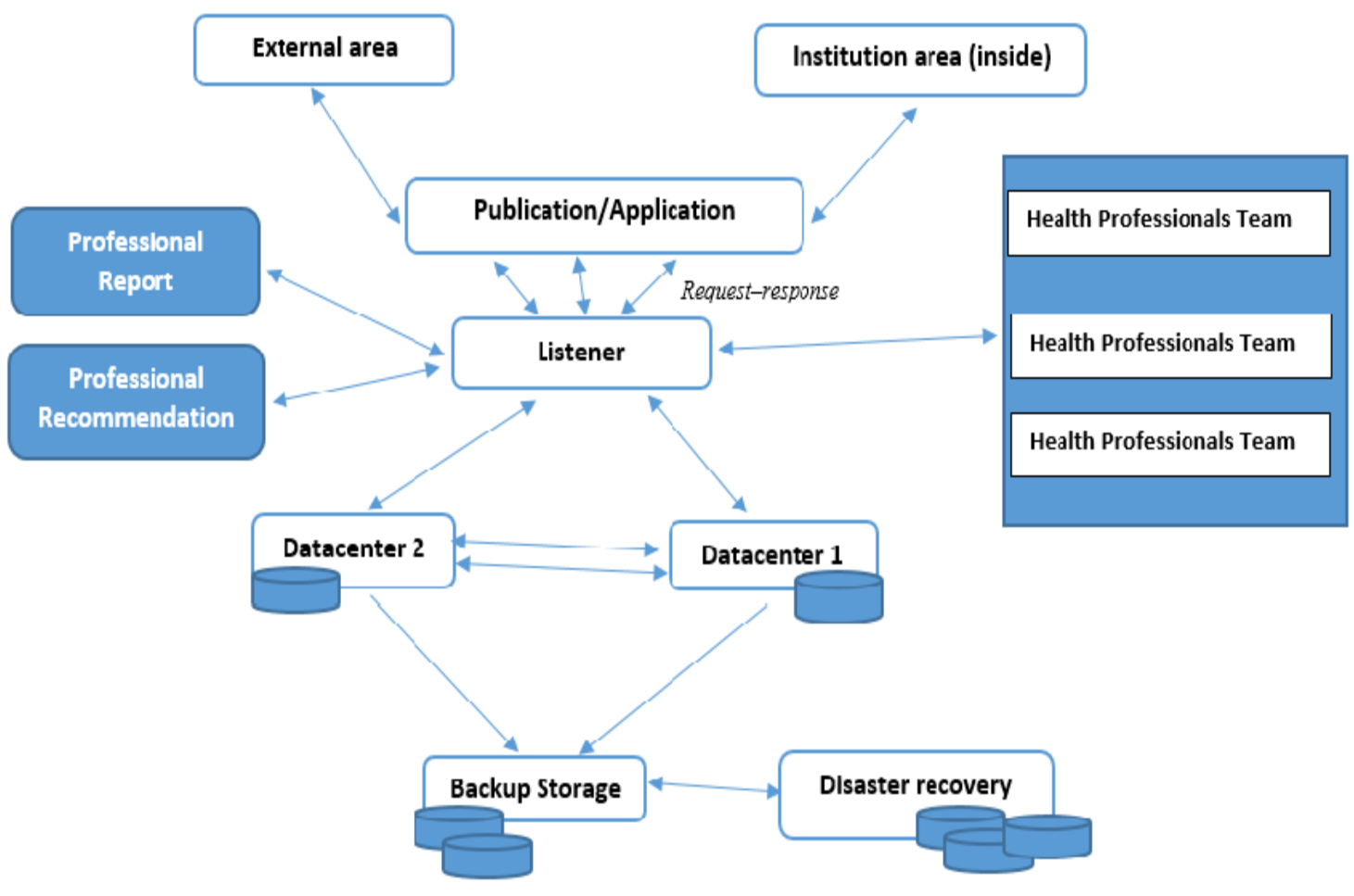

Data Center

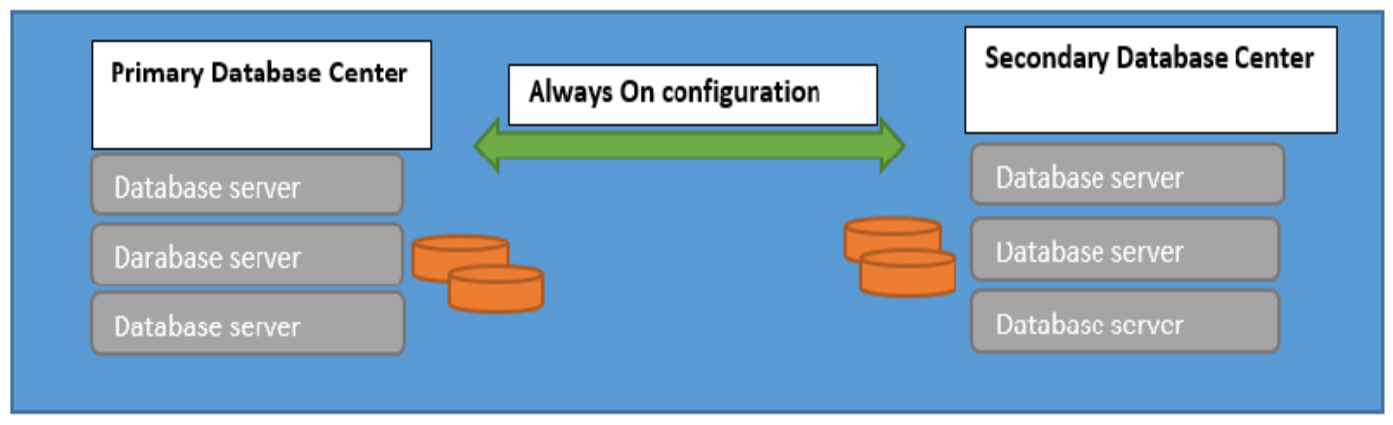

Figure 23. Disaster Recovery

Database AlwaysOn Configuration - helps the services to be uninterrupted, meaning that communication between end-users and the information base will be possible for each one. For this purpose, functionality, accuracy, speed, security and availability are very important components. SQL Server AlwaysOn offers a high availability and disaster recovery solution for SQL Server. Used in SQL Server as a combination of high availability and disaster recovery, this technology has begun to evolve and for this purpose has been named as HADR: 
- H-High

- A-Available

- D-Disaster

- R-Recovery

AlwaysOn is based on:

- Database Mirroring and

- Failover Clustering.

Microsoft cluster technology is used to protect against three types of failures:

- For the failure of applications and services,

- Hardware system failures,

- Failure of sites in multi-site organizations that may occur due to power cuts, communications, or natural disasters.

AlwaysOn does not make use of new technology as a whole but makes the most effective use of existing technologies that have been tested. It primarily aims to provide more control in order to reach a higher scale of availability.

Listener check the database that searches for the primary server for receiving requests and also redirects the request. The listener configuration enables us to access the frontend apps that access the database by accessing the listener because the listener chooses the primary server. The listener redirects the requests that come from different applications and drives them to the primary server whatever it is. So even if the primary server fails, or when servers switch roles from primary to secondary or vice versa, the application or user does not break away from the database, and there are no lost information packets.

Database Backup in the data architecture will have a backup strategy if information is to be restored to a new location or for monitoring or in case of failures of maintenance.

Through the backup strategy, information can be returned at certain time intervals where even the lost data can be repaired. Configuration of the backup is done automatically by 
revoking Sql agent for storing backups. During configuration, the choice is made when and where a backup copy of the primary or secondary server should be set up. This is of particular importance in maintaining and improving the database performance during the creation of the backup copy.

Disaster recovery will be a system independent of the primary system in a different geographic location in physical or natural disasters; this system must be ready to access and take the role of the primary system as soon as possible. Disaster recovery should be updated with the latest data on regular daily basis or even more often depending on what type of information is found in the databases. The Figure 23 shows the primary data center and the disaster recovery data center where the synchronization between of data centers is done synchronously and asynchronously. Synchronous between the databases in the primary datacenter and asynchronously between the primary and the disaster datacenter.

\subsubsection{Data security}

Data security has the highest priority, because health records are classified and confidential, data encryption should be applied to the database. Backup copies must be also encrypted. According to EU rules, any information identifying an individual should be protected and coded against free reading and understanding (GDPR). Encryption can be done in several methods by deleting the rules and procedures that differ from which method is omitted. The minimum to be implemented is Encryption Data Transparency. Example: Encryption TDE ${ }^{1}$ should be created: for example certificate and database encryption key in combination with certificate.

Among others Always Encryption enables information to be generated from the database even it is transmitted over the network from the database to the client and is deciphered only in the relevant client application, which means that the data is also protected from administrators of the system.

\footnotetext{
${ }^{1}$ TDE - Transparent Data Encryption
} 


\subsection{Technology Architecture}

To propose a technological architecture solution for the eHealth information system, it is very important to emphasize the life cycle of different entities and their interactions in the ecosystem. The longevity of equipment and data lifetime is a critical part of the understanding the technological architecture at every level. Architecture can be analyzed in three relevant aspects that meet the requirements and objectives outlined earlier. The three aspects to be addressed during the technical architecture development are as follows:

1. Access Layer the data collection and enhancer in this regard is related to how data is collected from end-user utilities. Data can be transmitted from various electronic devices and therefore we propose architecture layer for end-devices that can be used for both application and technical aspects, including communication capacity and protocol.

2. Network Connection, the focus for this aspect is related to how devices are connected to the ecosystem. There will be a review of the most advanced communication technologies inside and outside the system, by choosing adequate communication between end-devices and data storage devices, communication between mobile devices and the system using the latest technologies like 4G, 5G LAN, WAN (Oleshchuk \& Fensli, 2011).

3. Access Security and Privacy treatment are one of special importance in this architecture where confidentiality will also be treated. As healthcare data will be communicated through wireless networks, efforts must be made to preserve confidentiality and privacy. Privacy includes user's right to control the collection and distribution of personal information, while security is the protection of user information from unauthorized access. To carry out the assessment of security architecture for eHealth, we will focus on the latest perspectives and assess the security challenges we have to cope with in each of the key areas, including: end-users and their access to internal networks, private cloud services, partners, internet service provider, health system data held on web platforms, and implications that may occur from standards applied to wireless devices.

The purpose of technological architecture is to propose a functional architecture for a healthcare system focused on the real-time and periodic interaction with patients through information technologies. Architecture components enable functionality, the specification of 
technological devices, such as the infrastructure network, servers, sensors, web platforms, mobile devices and applications, communication protocols, data storage standards, and virtual data management systems will be based on the functional requirements.

The Enterprise Architecture approach - amongst others - is built on the principles of interoperability and re-usability. Meeting with the mentioned criteria architecture repository plays vital role. Repository consists of many components, from our point of view the building blocks, guidelines, protocols are important. In the Appendix we give a high level summary of the components which are used for development eHealth technology architecture, we will not go into details of the protocols, topology and other very technical elements. Figure 24 illustrates the technological architecture which includes all the communication and interaction between the access level, networks, data, and security. 


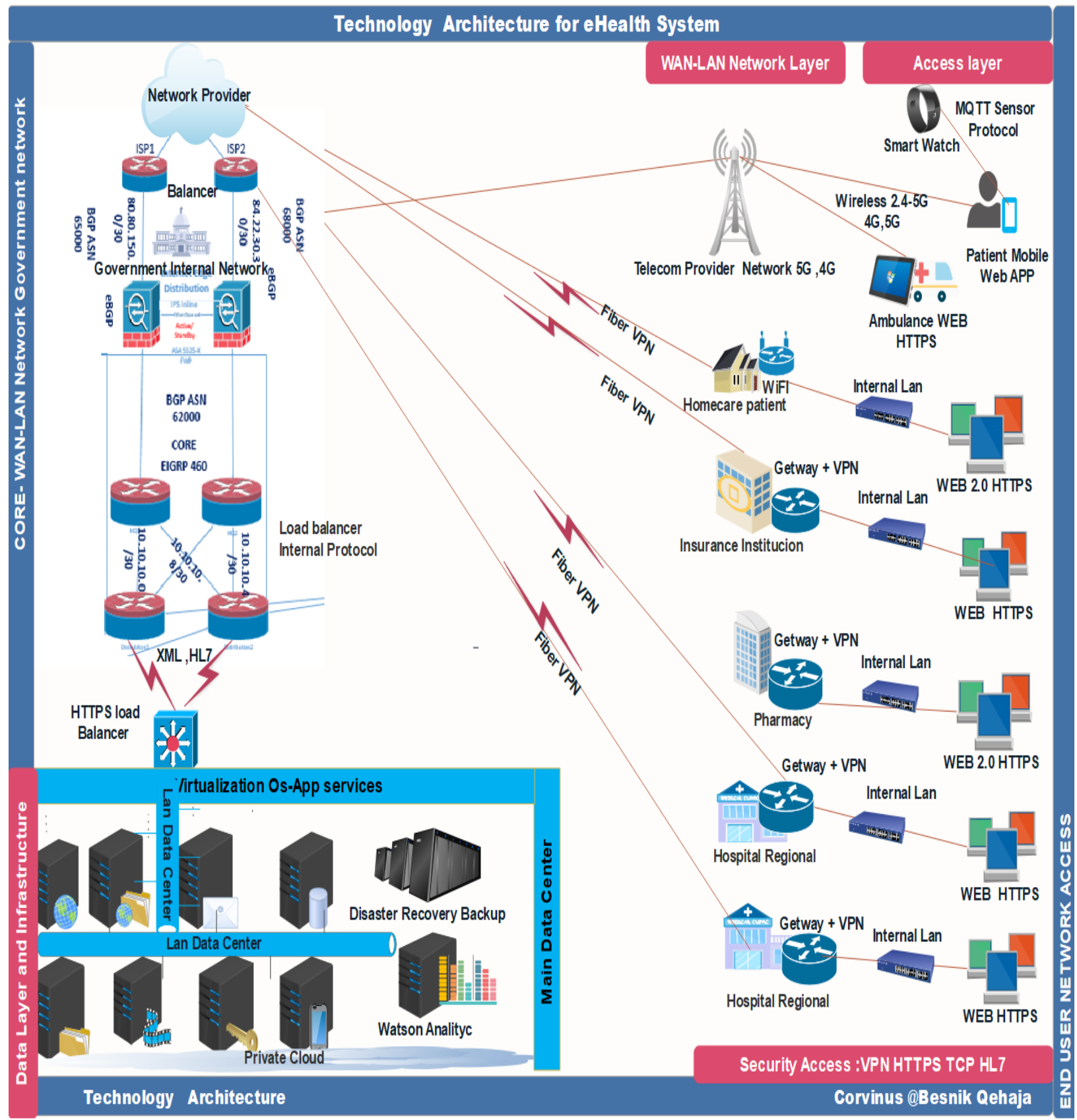

Figure 24. Technology Architecture

In technological architecture as a primary objective, an interdependent and functional topology has been proposed in order to support / provide the necessary services for health institutions. Many factors are considered in this architectural solution, such as the definition of network topology, hardware selection and software platforms, as well as the ability to carefully configure each component. All of these elements are essential in addressing the 
requirements for a sustainable health system. Therefore, considering these elements, the Access Level, Network (WAN) Services, as well as the Data Security and Data System are designed based on the following specifications:

- The architecture based on location infrastructure and the organizational structure of the institution based on geographic level and the existing network of institutions

○ Functional and technical requirements for the dedicated service of the institution.

$\circ$ The medium and long-term development trends and technology development trends.

- Compliance with international standards for designing and securing network and data.

- The architecture supports the structuring or creation of specific user groups based on the organizational structure of the institution and the services of groups for external communication from the institution.

- The network and services are compatible with the existing infrastructure and enable the provision of real-time and uninterrupted services (24/7).

○ Inbound network security (intranet-vpn) and external attacks (internet).

○ The security of the services from the outside (Internet) facing the web services, medical data.

\subsection{Conclusion}

Taking into account all the proposals at all levels, namely Application Architecture, Data Architecture and Technology Architecture their standardized logical and physical connection, I believe that the proposed solution is realistic. With that being said, it is a realistic and achievable solution if the implementation involves the major stakeholders mentioned and presented above. In broad terms, the architecture meets the requirements to a certain degree and extent. But this does not affect the main architectural masterpiece and proposed solution. Furthermore, in the upcoming sections below, the implementation of the proposed solution and its architecture will be described in great detail from the baseline to the target architecture; how the proposed solution can be put in practice. 
PART THREE 


\section{Roadmap}

The implementation proposal of eHealth architecture requires a harmonized roadmap of planning activities in all types of architecture. In this chapter, we highlight only the part of the roadmap, which ends with the implementation. Initially it should be analyzed the infrastructural aspect in the current situation of health institutions at state and local level. A very important aspect is the timeframe for all activities that can be carried out within the specified timeframe.

From this perspective, having enough resources is of key importance to initiate with the eHealth system building, including human and technological resources, and how stakeholders are willing to be part of the development of the system. Throughout the proposed architectural recommendation, the scope starts from the current state to an adequate solution that covers the health system requirements for a stand-alone, sustainable version, in conformation with WHO standards. The proposed planning and roadmap will cover system design, development implementation, deployment up to the activities of system testing and user training. Below we will see the activities both graphically presented the activities together with the time, as well as the description of each activity. The working method for system development will be based on the TOGAF framework using ADM model as well as the combination agile and waterfall system development methodology.

\subsection{Requirement phase}

Initially a functional requirement analysis is performed for each level and should be divided into functional requirements, system requirements, and non-functional requirements.

The second phase will be Design of Architecture it is divided into three main parts, the design of a computer network in a logical and physical aspect. Designing databases, creating tables and connection between them so that the data is organized for each database starting with the

Medicine-center Database, Pharmacy Database, Prescription-Center Database, Health Insurance Database, Healthcare Provider Database, Image Data store and the integration of the Civil Registration Database. 
Web design and mobile applications as well as technology architecture based on SOA should show how to handle the request and return to the code, GUI design for the entire system will determine what view the system will have when it will be used by users and stakeholders.

The design of middleware is concerned with the architecture principle: how databases interact with the applications. Design at this stage includes all services initiated by users starting with Patient Portal Doctors' Portal, Emergency services, Insurance portal, Pharmacy portal, Monitoring Applications, Backend systems. The design of the security should focus on at which levels must carefully manage data security and communication security by analyzing and implementing three-tier application, data and technology security.

\subsection{Development phase}

In the course of development it is of significant importance that the team of developers shall be appointed by staff who are specialized and have work experience at the three levels. The staff will consist of the project manager, a group of software developers, computer network engineers, security experts, and information system experts. Each team should keep up to date, so that the three-tier architecture has interdependence during development and each stage needs to be tested in small parts.

Software development will be the part of architecture implementation, divided into different parts: coding, development of system modules and integration of the system modules. At this point, all the modules are joined and completing the system, the initial performance testing will only be performed on the written code and the purpose is finding errors.

The next step is adapting the IBM Watson analytical system and linking to databases, developing and applying APIs for real-time communication with the help of web services.

Developing the network topology and restarting the existing network starts from the data center. First, we need to see which state network is safer and need to distribute more reliable ISPs in the market for collaboration. Second and foremost, you embark on with a close observation of the current infrastructure as it fits to the requirements, then begin to replace the appropriate devices and configurations at each level ranging from institutions and other stakeholders to the data center. The network needs to be tested in advance using simulation software before going live on the system as this feature exists and a portion of the services 
can be tested within the network. Configure network services ranging from Voice over IP through H.245, IPsec, EGRP, BGP, OSPF.

Developing disaster recovery and AlwaysOn system, linking to core databases and creating a second backup system that supports database architecture and provides security in case of failure or other anomalies during work. Configuration and backup options for each level of architecture, network redundancy and other safety measures will close this step.

\subsection{Testing phase}

Testing means a thorough check of performance, errors indicate the points where the system fails, forget to meet the user's request correctly. Documentation of the issues give a good starting point where the system shows weaknesses and inaccuracies in the performance testing. The document will be delivered to the developers and they will resolve the problems. The general computer network should be tested using virtual simulation software as a Packet Tracer simulator and GNS3 before the system is placed on the system to do specific tests ranging from Data Center to the patient or stakeholders.

The security aspects of network applications must be tested by testing VPN, IPsec access protocols SSL, TCP HTTPS, Firewall security, Digital Key Infrastructure Digital Certificate and databases by applying GDPR compliant data encryption and testing using cyber-fish attack methods, dos, web penetration etc. Application and introduction of eHealth standards HL7, Digital Imaging, ISO / TC 215, ISO / IEEE 11073 is also part of the testing activities. Service application scenarios should be tested for each of the parties to see how the user interacts before starting the system and receives user feedback for any specific request and carry out changes to the system. Below you find a visual view of activities including time and details (Figure 25, Figure 26 and Figure 27). 


\begin{tabular}{|c|c|c|c|c|c|c|c|c|c|}
\hline \multirow{2}{*}{ Version } & \multirow{2}{*}{ BASELINE 1.0} & \multirow{2}{*}{ Duration } & \multicolumn{7}{|c|}{$2020 / 2 / 29$} \\
\hline & & & $2020 / 3 / 1$ & $2020 / 4 / 1$ & $2020 / 5 / 1$ & $2020 / 6 / 1$ & $2020 / 711$ & $2020 / 8 / 1$ & 2020/9/1 \\
\hline 1.0 & Analysis of requirements & $7.0 \mathrm{~d}$. & & & & & & & \\
\hline 1 & Functional requirements & $0.0 \mathrm{~d}$ & $\boldsymbol{\Sigma}$ & & & & & & \\
\hline 3 & Meeting with Stakeholder & $0.0 \mathrm{~d}$ & $\Sigma$ & & & & & & \\
\hline 4 & System Requirements & $0.0 \mathrm{~d}$ & $\boldsymbol{\Sigma}$ & & & & & & \\
\hline 5 & Design of architecture & $8.0 \mathrm{~d}$ & & & & & & & \\
\hline 6 & Network Design & $2.0 \mathrm{~d}$ & $\boldsymbol{\Sigma}$ & & & & & & \\
\hline 7 & Logic Topology Network & $2.0 \mathrm{~d}$ & $\Sigma$ & & & & & & \\
\hline 8 & Physical topology Network & $4.0 \mathrm{~d}$ & $\boldsymbol{\Sigma}$ & & & & & & \\
\hline 9 & Design Database & $4.0 \mathrm{~d}$ & $\Sigma$ & & & & & & \\
\hline 10 & Medicine Centre Database & $4.0 \mathrm{~d}$ & $\Sigma$ & & & & & & \\
\hline 11 & Pharmacy Database & $4.0 \mathrm{~d}$ & $\Sigma$ & & & & & & \\
\hline 12 & Image Datastore & $4.0 \mathrm{~d}$ & $\Sigma$ & & & & & & \\
\hline 13 & Civil Registration Database & $4.0 \mathrm{~d}$ & $\Sigma$ & & & & & & \\
\hline 14 & Monitoring Applications and Web Desing & $4.0 \mathrm{~d}$ & $\Sigma$ & & & & 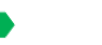 & & \\
\hline 15 & Design GUl & $4.0 \mathrm{~d}$ & $\Sigma$ & & & & & & \\
\hline 16 & Design Middleware & $4.0 \mathrm{~d}$ & $\Sigma$ & & & & 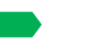 & & \\
\hline 17 & Design Portal Patient & $4.0 \mathrm{~d}$. & $\boldsymbol{\Sigma}$ & & & & 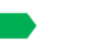 & & \\
\hline 18 & Design Doctor Portal & $4.0 \mathrm{~d}$. & $\boldsymbol{\Sigma}$ & & & & & & \\
\hline 19 & Design Emergency services & $4.0 \mathrm{~d}$. & $\boldsymbol{\Sigma}$ & & & & & & \\
\hline 20 & Design Insurance and Pharmacy portal & $4.0 \mathrm{~d}$ & $\mathbf{z}$ & & & & & & \\
\hline 21 & Design Backend system & $4.0 \mathrm{~d}$ & $\boldsymbol{\Sigma}$ & & & & & & \\
\hline 22 & Design Monitoring Applications & $4.0 \mathrm{~d}$ & & & & & & & \\
\hline
\end{tabular}

Figure 25. Road Map activity implementation (part one) 


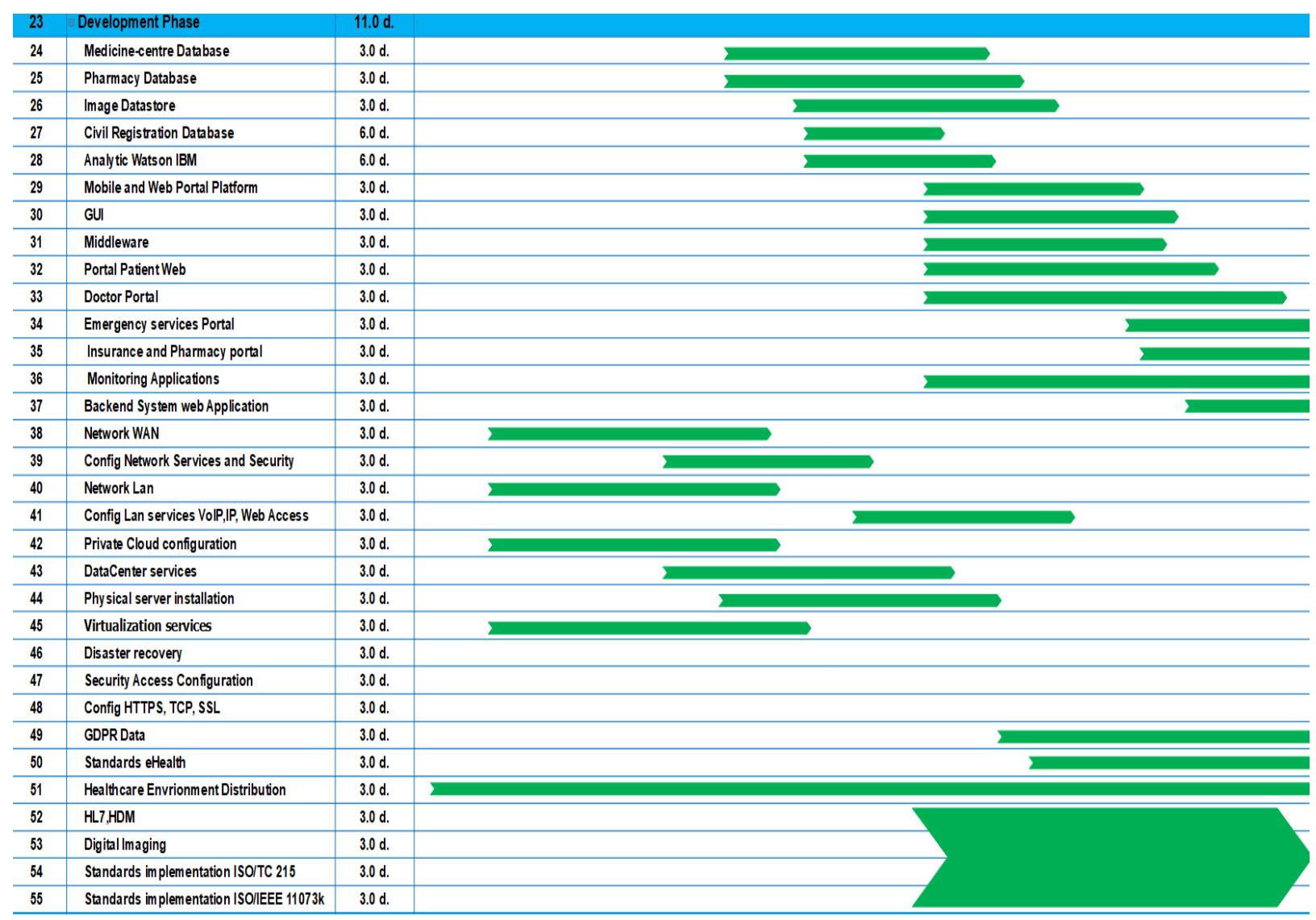

Figure 26. Road Map activity implementation (part two)

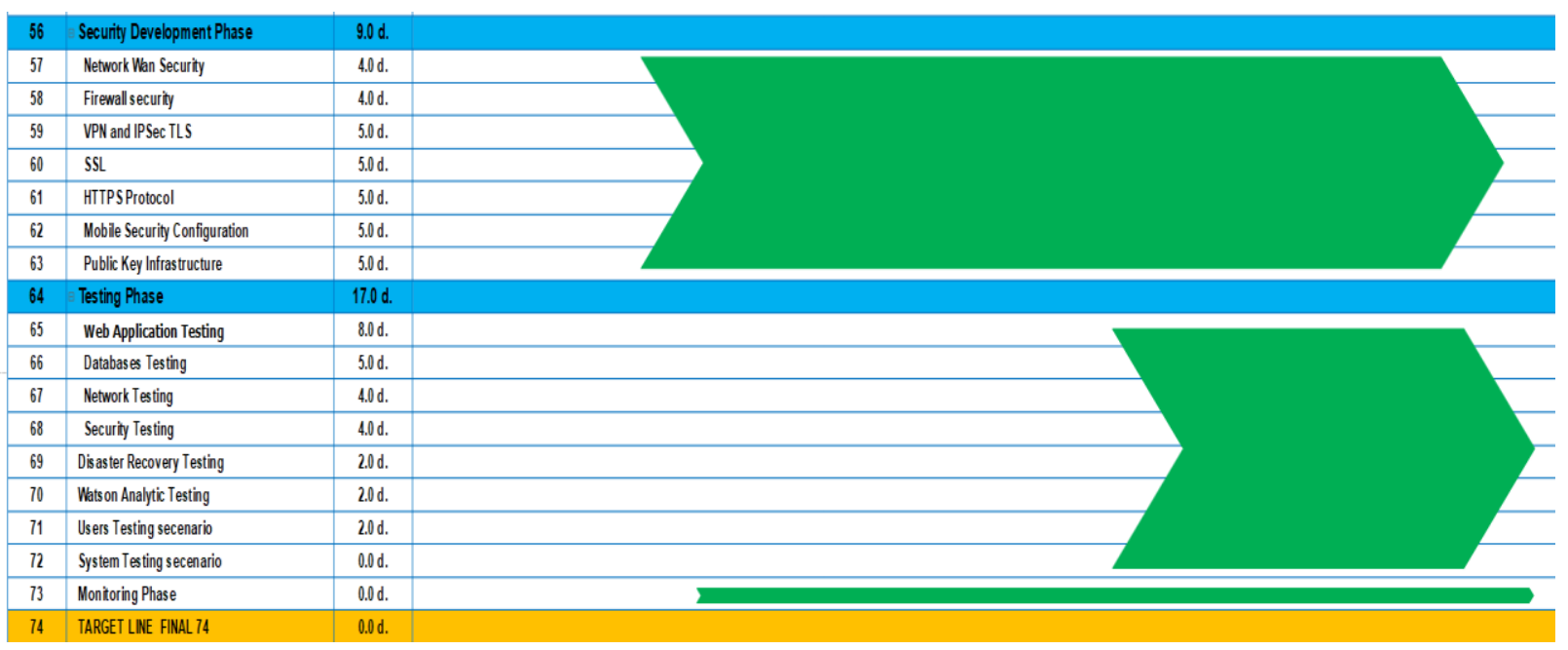

Figure 27. Road Map activity implementation (part three) 


\section{Implementation Strategy}

Based on the overview of how the architecture of the eHealth system should be developed in realistic terms, it is very important to implement the strategy and its vision.

Strategic implementation will be based on a clear overview of the eHealth architecture development process, where the objectives and indicators that are involved in the process are measured, reviewed and defined: The objectives should be pursued by the Ministry of Health's requirements for their vision and mission, the WHO Strategy's view, social, legislative, and stakeholder demands.

In the framework of these basic aspects and system development cycle as well as the barriers during the implementation process, we create the basis for starting the drafting phase and adopting the Implementation Strategy.

During the development of technological architecture the implementation time was envisaged, it was estimated in 2 years from the start of implementation. Additional 6 months needed for training, awareness raising campaigns, overcome barriers. In the design phase of the above proposed architecture we have calculated all aspects of development in detail, and for us it gives more confidence that the implementation strategy will not have any major obstacles. Therefore, referring to planning in all aspects involving human and technological resources, an action plan for the implementation of the eHealth system has been set.

\subsection{Preliminary and Planning Tasks}

The action plan is foreseen to be divided into five phases so that the implementation strategy is as realistic as possible. The stages are divided into the following steps:

- Initially, the first phase is the initiation of the project, primarily the vision of the architecture, the feasibility analysis, which will include the justification of investment and project budgeting, the stakeholder meeting where will be discussed who and what will benefit from this system, the team meeting and formation of the Steering Committee. It will also analyze the current infrastructure situation and setting up the main objectives.

- The second phase is planning. This consists of a technology analysis, cost analysis, procurement procedures (if needed), and budget planning. Planning system development 
ends this phase by addressing each implementation phase that is detailed in the Roadmap section.

- The third stage is to identify the requirements that need to be meet during system development, such as applying a unique identification number for system access and privilege sharing.

- The fourth stage is the identification and stipulation of risks that involves system analysis and identifies the risks that may occur and identifies methods and ways of preventing them so that the system does not fail and does not deviate from planned processes and expenditures.

- The fifth phase is the treatment of the human resources plan for system development and management, as the distribution of tasks and responsibilities. Selection of highly specialized and experienced staff at the three levels of development and managerial aspects.

\subsection{Implementation Phase}

Referring to the three-level architecture proposal and eHealth development steps, it is very important that during the implementation of each phase, time and resources should be allocated, as well as prioritization of tasks. Starting from system requirements, system design, system development, system testing is expected to be tested in the 3 main centers that will be piloted before implementation is extended across the country. In addition, elapsed time should be considered in proportion to the risk calculation that is anticipated during the start-up phase.

During the implementation phase, it is important to create a group for the implementation audit, another group for auditing the standardization of technical equipment and the standards of medical records. It is important that during the implementation a group should monitor the tendering and contracting process of the technical specifications of the equipment and software and their maintenance. It is also envisaged a group that reviews data security and privacy measures in connection with regulations. 
A group should treat and approve key beneficiaries at all levels of private and public health care about the services, obligations provided by the eHealth system. In addition, the costing and pricing aspects should be taken into consideration. This strategy envisages continuous monitoring of activities and their timely reporting to supervisors, and monitoring in the technological aspect where a phase of upgrading and monitoring of the system will be established throughout its use.

\subsection{Stakeholders' Awareness and Motivation}

\subsubsection{Motivation and awareness raising of citizens}

After the completion of the system development and testing phase, the training of users groups is foreseen, including their peers in the pilot institutions. Meetings and trainings for hospitals management staff, as well as providing technical assistance to each hospital until IT staff of hospitals are able to operate independently.

After testing the system with staff and hospitals, awareness raising of patients and other users is envisaged through an awareness campaign that would include public meetings with citizens and staff training in the respective hospitals. Trainings for the use of eHealth information system cover the use of key communication tools, which are used, by various interest groups as well as campaigns are needed through platforms in social media, which provide quick and efficient information for all interest groups. Awareness campaign will be supported by manuals and extended use of video messages (e.g. you tuber influencers).

A very applicable method for citizens' awareness raising is sponsorship on social networks by creating filters for different age groups where ads are filtered based on age and gives each group an opportunity to see the benefits that the system offers if they use eHealth Information System.

Another efficient method of citizen awareness raising is messaging on mobile telephones from Telekom of Kosovo; these messages encourage citizens to appear at the health service centers to obtain additional information about the eHealth information system and the impetus that this system brings. One of the most important components within the awareness raising campaign for citizens will be the aspect of maintaining privacy and data security when using different eHealth platform platforms. In this context, citizens will be provided with an 
explanation and legal guarantee based on the legislation in force as well as the argumentation in the technology perspective that their data will be held accountable and punctual.

\subsubsection{Encouragement of insurance organization}

Health insurance institutions are encouraged to use the eHealth Information System, so that through this system they will reduce the costs of citizen's medical treatment that are included within the health insurance systems.

During the involvement of insurance companies will be explained in detail how this system will positively affect the health of their clients as well as reduce time and visits to administrative services at healthcare centers that are potentially visited by their customers. the campaign concerned with encouragement of insurance companies, will create them with the opportunity of providing cooperation for awareness campaigns regarding the use of the eHealth system.

\subsubsection{Cost and Return on Investment}

In the literature and in the indicator section, it is argued that the implementation of the eHealth system plays an important role in cost reduction and return on investment. In Kosovo, health financing is largely managed at the central level with very limited involvement and very low autonomy of health institutions. The budget is designed based on past spending, without any correlation with the needs of the population and without a strategy of priorities. In 2017 and 2018, the ministry has spent over 92\% of the final budget or $€ 57,567,776$. However, budget use remains unsatisfactory, where only $€ 11,354,000$ million is invested for capital investment and there is still no proper information system (Revizije, 2017).

According to the Audit Report (Revizije, 2017) in 2017 and 2018, the registration of the purchase of drugs has made it difficult to track the supplies and the management of medicines have noticed that the drugs purchased for the Essential List in the amount of $€ 1,431,071$ were not recorded in the absence of software has created direct damage to the budget. Whereas, the treatment of patients outside public institutions for the period 2017/2018 amounted to $€ 14,350,049$. As elaborated in the section of the current situation, both public and private budget expenditures are large, the increase in mortality in Kosovo is still evident. 
Therefore, these are pointed out some notable indicators that investing in an eHealth information system is more than necessary and the return on investments is expected to be high. Investing in such an eHealth project justifies the cost of investing since in many aspects the architecture and the proposed system play a key role in cost reduction at all stakeholders, as well as increase the efficiency of health care services is expected. The justification for the investment costs as well as the role of each of the above proposed collection levels is provided below:

- Patients and doctors through mobile and Web applications are fed with data, like registration of a meeting with a physician and payment, reduces the waiting time and helps coordinating processes and allocating resources better.

- Distance communication reduces visits to hospitals, saves patient waiting time, allows having remote contact with the doctor, and enables monitoring patient's health in real time.

- The doctor manages time better and decision making is more efficient, access to patient records is faster, more detailed and more reliable.

- Through this medical-patient communication, the system operates $24 / 7$ and also open for emergency services.

- The eHealth system also enables efficient management of drug prescriptions, enables monitoring and making sound decisions to avoid the excessive drug consumption.

Table 1 explains the eHealth information system lowers the cost in many respects: improving the prevalence, reduced waste during the visit, reduced prehospitalization, less physical contacts, improved managerial process. 


\begin{tabular}{|c|c|c|c|c|c|}
\hline Cost saved & $\begin{array}{l}\text { Improved } \\
\text { prevetion }\end{array}$ & $\begin{array}{l}\text { Reduced time } \\
\text { ëaste during } \\
\text { visit }\end{array}$ & $\begin{array}{l}\text { Reduced } \\
\text { rehospitalisation }\end{array}$ & $\begin{array}{l}\text { Less physical } \\
\text { conctacts }\end{array}$ & $\begin{array}{l}\text { Improved } \\
\text { managerial } \\
\text { process }\end{array}$ \\
\hline Data quality & High & High & High & High & High \\
\hline Data capture & High & Medium & High & High & High \\
\hline $\begin{array}{l}\text { Patient portal and } \\
\text { doctor }\end{array}$ & High & High & High & High & Medium \\
\hline $\begin{array}{l}\text { Managing logistic } \\
\text { process }\end{array}$ & Medium & High & High & High & High \\
\hline $\begin{array}{l}\text { Clinical decision } \\
\text { supported by } \\
\text { Watson analytic }\end{array}$ & High & High & High & High & High \\
\hline $\begin{array}{l}\text { Healthcare } \\
\text { stakeholders }\end{array}$ & High & High & Medium & High & High \\
\hline $\begin{array}{l}\text { Monitoring of } \\
\text { health services }\end{array}$ & High & High & High & High & High \\
\hline Impact & Low & Medium & High & & \\
\hline
\end{tabular}

Table 1. Key Performance Indicators for eHealth

\subsubsection{Investment indicator}

If we rely on the total state budget allocated to healthcare annually, which is $€ 57,567,776$ of which we allocate only $3 \%$ for the development and implementation of the eHealth system, then we can build a healthcare system which in the future will reduce the cost each year and will not need additional investment (National Audit, 2017a). 


\subsubsection{Communication infrastructure}

Since the war ended in 2001 Kosovo is in favor of network communication. The communication network has been extended throughout the whole territory of Kosovo, which is now a stable network and only needs to be restructured and properly configured with some additional investment in certain hospitals.

About $85 \%$ of the population uses the Internet via mobile or computer devices. Coverage of Kosovo's territory is about $98 \%$ with $4 \mathrm{G}$ cellular networks and in the next two years it is anticipated to be transferred to 5G. Human resources for expert developers are enough and the minimum wage for an engineer is about 800 to 1200 Euro, so investment in staff is not that big. With a better management and division of responsibilities and tasks, all stakeholders (especially IT in hospitals) can be involved and the burden on human and technological resources is dispersed proportionally (KAS, 2017a).

The Watson analytic system is a system that can be purchased and the price of licenses is very cheap, about $\$ 80$ per month, in proportion to the function it performs. Thus reconfiguring existing equipment, using the best practices of international standardized systems reduces the cost of direct investment. On the other hand in favor of cost reduction is the virtualization of services by creating private cloud, through virtualization we reduce the cost of purchasing additional devices as servers, routers, etc. In the existing center of the Ministry of Health, we can use most of the equipment only to allow for a great reconfiguration and investment using the method of verifying the equipment and services. Through ISP providers we facilitate access to each hospital, where prices are extremely cheap on dedicated lines around $€ 50$ for a speed of $200 \mathrm{Mbps}$, this is also a good way of reducing the cost of communication between the hospital and the data center, especially when it is in rural areas. Taking into account all the indicators, both in terms of technology and in terms

of human resources, we think that it is worth such an investment, because this investment comes back both in terms of improving health and improving services for all parties of interest (KAS, 2017a). 


\section{Research Methodology and Interview Questions}

\subsection{Research Methodology}

Research methodology according to Creswell (Abdusamadovich \& Sciences, 2013) is defined as general guideline for solving a problem or a systematic way of solving problem through design of novel solution. There are different kinds of research approaches; these include qualitative approach, quantitative approach, design science approach and mixed approach (mixture of qualitative methods and quantitative methods).

In this study, a combination of three methods is used because of the nature our research questions that require multiple methods to get them answered. Combining methods offers great promise on flexibility of the research and draw strengths from multiple methods, therefore allow them to answer questions in a broader way that are not confined to only one method.

The objective is to understand the challenges and propose technology-based solutions that can improve the data flow between different levels of healthcare systems. We further review the available technologies for data collection and evaluate their suitability in improving healthcare system in Kosovo.

1. Through literature review and qualitative study, we study health data flow in healthcare systems and challenges associated with healthcare system.

2. Through case studies, we use the same mix of quantitative and qualitative methods evaluating performance of system; we use similar key performance metrics which already have been used in two different project, in two different countries.

3. In the surveys (interviews), I intend to use the same mix of quantitative and qualitative methods, too.

The literature review revealed a deeper understanding of health systems in different countries. Case studies from different countries served as a base to research, analyzing the innovation, development, benefits, advantages, strengths, also the weaknesses and potential disadvantages of these systems. By understanding and analyzing the above-mentioned 
components, I am able to propose a real-time patient monitoring eHealth system, knowing that health sector face with expensive and complex services, which have a major impact on the economy and the everyday life of people.

During my research, I explored the relevant literature addressing the existing technologies, the applied information systems standards, a big variety of applications, and especially applications designed for mobile devices, including the possibility of interconnection with wearable and implanted body sensors. The purpose of the literature review and research is to confront the three conditions by using the existing ICT components corresponding to technical and semantic interaction within the healthcare processes.

I outlined the architecture of Real-time Patient Monitoring eHealth System (RPMeHS) according to the corresponding literature and particularly to my ICT knowledge. The next step is to verify, validate and justify the proposal.

The validation process aims to evaluate the functionality and usability of the proposed RPMeHS architecture using qualitative method. Considering the small number of working applications, the high dependency on awareness, willingness and regulatory barriers conducting thoughtful personal interviews looks the most appropriate method to be applied.

The survey strategy is associated with the deductive approach, and it is an appropriate procedure to answer questions like who, what, where, and how is doing. The questions in the following sections, give the skeleton of the interviews, however some deviation from them happened time to time.

\subsection{Selecting Target Groups and the Planning the Interviews}

This research is primarily based on the five regions of the Republic of Kosovo. The data and opinions were taken from six hospitals, including three well-known international healthcare centers, including the Center of University Clinic of Kosovo (QKUK), which plays a central role in the tertiary healthcare in Kosovo. Other hospitals are of regional character, like Gjilan Hospital in the eastern region, Regional Hospital of Prizren in the south, Regional Hospital of Peja in the west, the Regional Health Center in Kamenica, as well as the regional hospital in Ferizaj. These hospitals especially considered monitoring as essential part of their core 
activity. In rural areas and in small villages monitoring is particularly important for the secondary health care, too.

Creating different groups of interviewees is based on the separate roles what they have in the system design, development, implementation and operation. From this point of view, the following groups were defined:

1. Medical staff (doctors, nurses)

2. Patients

3. ICT experts

\subsubsection{Interviews with medical personnel}

In the first stage of the interview, I visited each of the selected institutions and conducted the interview with selected participants (for the detailed interview plan see Appendix 1). They were contacted by phone in advance and they were informed about the purpose and method of the interview. Their 'consent informed' approval for the interview was obtained as well. All participants revealed their keen interest and willingness to cooperate and participate in the interviews.

The purpose of data collection via personal interviews is to understand better the existing working conditions of doctors, nurses and administrators. During the interview I focused on learning about their views on digitalization in medical services, collecting the role-specific requirements, finally getting some input in regard to their willingness accepting digital system as it is outlined in the thesis. I also pay attention to the fact they are not ICT experts.

\subsubsection{Interviews with group of patients}

The patients intended to be monitored were divided into three groups, 5-10 patient of each, and the interviews for the sake of empirical research have been organized as follows:

- First 'Chronic' group - Male and female patients aging from 30 to 65, with chronic disease. This type of patients does not need active long-term care at the hospital. They get treated at home but need regular checkups at the hospital. They follow medical treatment, medical procedures and take the prescribed drugs as advised by the. 
- Second 'Need attention' Group - This group consists of recently hospitalized patients, their age varies between 30 to 50 years. They had an intervention, especially surgeries like arthroscopic or heart surgeries in the past. This group of patients usually spend time between three to six months (as advised by the doctor), in the rehabilitation center of a hospital or in other specialized institutions. In ideal case, they are monitored on a real time basis.

- Third 'Healthy' Group - Members of the third group are healthy people and representing both genders: the males and females aged between 18 and 50. This group plays the role of a control group, but they can be also the beneficiaries of the system. It is assumed that in the following years the system will be enhanced with additional functionalities, to keep track of their health status, in order to prevent future diseases. Fitness sensors, fitness saloons, communities, tracking through smart watches or mobile devices and many more technologies will be used to keep an eye on this target group as well.

For the detailed interview plan, see Appendix 1.

\subsubsection{Interviews with ICT experts}

I performed interviews with computer scientists and information systems experts to obtain additional input to the thesis, in order to gain a better view and evaluation of the proposed information system architecture. Their assessments and were performed in three areas of information systems architecture: application, database, and technologies used for the proposed architecture. The questions were adapted for each interviewee type, depending on the field of expertise (for the detailed interview plan see Appendix 1).

\section{Validation}

The national health system is improving when the information system addresses national demands and problems. Therefore, the research focuses on the current situation and the objectives set out above. It is assumed the Information System Implementation Strategy (cf. Chapter 9) will respond appropriately to the demand articulated in the research questions.

The interviews gave the opportunity to me to confront the proposed architecture with the 
users' requirements, also to address the local and global barriers, implementation and deployment difficulties that they mentioned. From technical point of view, the ICT experts approved the basic concept of the proposed system both from architectural and operational context.

The detailed description of interviews is in the appendix, in this chapter I intend to summarize the most important and interesting findings.

Below Figure 28. is a visual structure of how all problems articulated in the research questions will be handled:

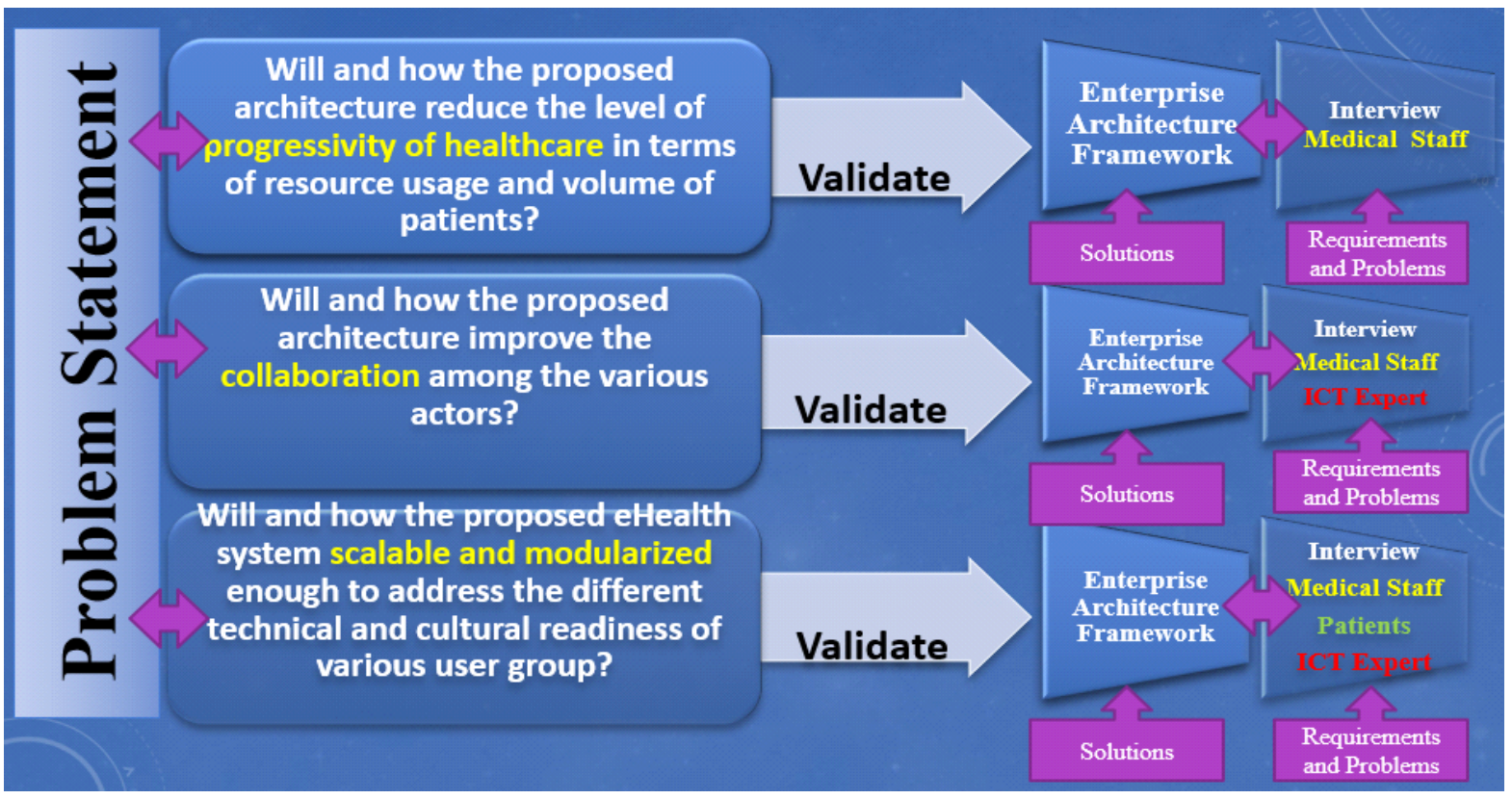

Figure 28. From problem to solution - validation

\subsection{Healthcare progressivity (RQ1)}

\section{"Will and how the proposed architecture reduces the level of progressivity of healthcare in terms of resource usage and volume of patients?}

\subsubsection{Problems and requirements}

As it was highlighted in the description of current situation regarding the treatment of patients, in most health institutions in Kosovo all treatment processes are still recorded in hardcopy paper. During the interviews with doctors, nurses and hospital managers, it was 
identified as a main problem. The lack of appropriate and up-to-date patients' data often leads to faulty or incorrect diagnosis, which obviously followed by more resource usage and requires more progressive care of patients (15.2.1), (15.2.2), (15.2.3) . Preventive measures for a specific patient are almost impossible, long-term patient monitoring is also impossible. Doctors mentioned that patients often visit two to three physicians a day to receive second opinion but their paper-based treatment history is kept secret, the other doctors cannot assess them (15.2.1). This phenomenon creates multiple workload and additional management cost of management (15.2.3).They also recommended access opportunity to the detailed patient history for the sake of a good diagnosis; possibly back to the patient's birth (15.2.1).

Nurses also pointed out the lack of patient data and inventory that is available at hospitals and the lack of a system that coordinates appointments with doctors and nurses, leads to many disadvantageous situation in patients' treatment and this directly affects their performance in work and health status of patients, too (15.2.2). Nurses during the interviews also commented that lack of communication with the patient after treatment leads to frequent repeats of the same diagnoses, as many patients travel from rural areas and access to and communication with health systems is not possible in cases of short or longer consultation depending on the patient's condition (15.2.2).

Hospital managers during interviews indicated that one of the problems they face is patient waiting time, averaging up to 20-30 minute, due to lack of data and a system of patient appointments. (15.2.3) The lack of such a system is causing problems in managing the services for treating patients also in the planning of human resources in hospitals. Interviews with hospital managers showed that lack of data on available staff, inventory costs, workload, and the number of patients in proportion to beds in depots (15.2.3). Currently the management staff process all the planning in the hospitals where they work based on the data of the previous month and where each month different and therefore different data is are reported in the management processes (15.2.3). Based on the requirements and problems outlined above, the proposed architecture at the application and data architecture level directly provides the answer to most of the problems addressed above by medical interviewees. 


\subsubsection{Proposed Solution}

The basic principle of Web platform-based Application Architecture in our proposal is to collect data from different users and make them accessible by different technological devices. The different applications proposed here have homogeneity; each application has access to different databases enabling different users to obtain different information.

In the suggested solution patient data retrieval takes places on Patient Web Portal, it is an application through which a patient can communicate with service providers, as the patient can access the patient portal and view information on patient profile, term, results of diagnosis, medical recommendations, prescription history, choice of doctors available. The portal also includes video-chat-bots, which allow a user to discuss treatment for a health problem, or administrative issues.

This information is essential for the medical staff, where based on the medical staff requirements identified during the interviews. The data required by staff also helps for realtime patient monitoring.

Also, according to the requirements of nurses and doctors the solution offered in our proposal is the Doctors' Portal, this solution is similar to Patient Portal, but data access and information services are more detailed and professionally personalized. As part of the Doctors Portal is the Tele-monitoring Module, which is specially created for the physician, which collects high volume of patient status data and many other information, especially when the patient has values different than normal, then the physician can react in real time. Among the important information are the patient's history of previous treatments, irregularities in the status of the sensors that monitor the patient, prescriptions, current treatments, and duration of treatment.

Based on the interviews the long waiting time for the basic treatment is high and the efficacy of services is not appropriate, (15.2.3) so the solution proposed through Monitoring Application aims to collect data from patients and the environment in which the patient livesThese data reduce the number of hospital visits and also discharges staff burdens doing direct check-ups of patients. Therefore, the main beneficiaries of monitoring services will be patients, while their relatives will be the secondary beneficiaries, as their families will save 
time and money by using remote monitoring. Monitoring services can be implemented in many ways: fixed sensors and removable sensors. Through the mobile app, patients can be monitored inside or at workplaces, or during sport activities, or even during leisure time. This app is intended to take preventive measures for non-sick patients but requires periodic treatment (checkup). Also doctors and nurses through the recommended Watson Analytics system by configuring APIs for specific patient monitoring cases enables them to monitor even more specific patients, especially Acute and Chronic ones. Monitoring application can be used to monitor specific groups of people who have a higher risk of becoming a patient, given their profile. Monitoring may include not only the key parameters that medical staff necessarily require but should also include the specifics of a healthy lifestyle, including physical activity, nutrition, and even some of parameters that indicate mental status.

Emergency Services application is an application designed to check the status of patients who are in critical condition, alerting medical staff in case of emergency. The scale of intervention is wide ranging from informing a doctor to providing on-site help. This application enables specific cases, especially patients in rural areas and during accidents requiring special intervention and health monitoring and helps to take preventive measures especially for Acute and Chronic patients.

Responding to the demands of nurses and physicians on medication and the status of providing adequate medication to patients (15.2.2). The Pharmacy Portal recommendation helps medical service providers and pharmacies send and receive prescription data through recommended platforms. The consumer (patient) will also be enrolled in the pharmacy record to receive the prescribed medication. An additional service that the Pharmacy Portal enables for both physician and patient is the control of drug interactions, as well as tracking consumption can be a good starting point in the fight against inappropriate medication manipulation, which is a key issue in Kosovo.

Using the Prescription Center Database, the prescription center database stores all the prescriptions that have been prescribed by healthcare provider. The healthcare provider stores all records of the diagnosis, treatment, and prescribed medication for each patient separately and sends prescriptions regularly to the prescription center. Prescription center transfer 
prescriptions to pharmacy database to check availability and in case out of stock suggests replacement of drugs. This database helps in better planning of the managerial staff regarding the planning for the medicines stock. Also based on hospital managers' requirements regarding inventory availability through the Medicine Center Database has a very close relationship with the pharmacy due to the fact that it controls all inventory currently available at pharmacies and liaise with pharmacies to know which drug is in their possession so that the correct drug can be found for patients in need.

Based on the requirements of hospital nurses and managers' opinion, (15.2.3, 15.2.3) the Hospital Resources Database contains data of available rooms, beds available in those rooms, current patient numbers and the number of new patients arriving. The healthcare provider always communicates with this database to check availability, and in the same way, using the database enables the healthcare provider to operate if the number of available rooms or beds is decreasing and make plans to cope with different load situations. This ability - to act in time - reduces waiting times and give patients a feasible solution for treatment at hospitals on different levels depending on their health situation.

\subsubsection{Reflection}

Looking at the problems and demands of the medical staff, on one hand we see a technologically significant solution based on my proposal that strongly correlates with the demands and problems of all users of the health system. At the same time, the medical staff responded positively that a digitalization of the health system is a big step ahead, compared with the traditional form of health services causing to them problems in performance and work efficiency. For most medical staff it remains a challenge to train older staff using modern technology to treat patients. Hence our application architecture proposal greatly increases the efficiency of the work of the medical staff and at the same time reduces the volume of patients in the health institutions.

\subsection{Collaboration (RQ2)}

' Will and how the proposed architecture improves the collaboration among the various actors?', 


\subsubsection{Problems and requirements}

Answering the first research question we found the main concern of the medical staff is the lack of patient data as a key problem (15.2.1) (15.2.2) (15.2.3). The second research question closely relates to the need for better communication between stakeholders, which is a key indicator in generating sufficient data for health staff and for all the other stakeholders working in health services.

During the interviews with the health staff, we noted that the lack of communication between the medical / non-medical staff and the patients has led to the lack of data and poor treatment. (15.2.1) (15.2.2). The interviewees requested that the data be grouped into laboratory data, imaging data, in order to better identify the patients' diagnosis, and to treat them better. Interviewees engaged in health-staffing also concluded that a patient must be treated with emergency when one of the vital values exceeds the median parameters, indicating that health care staff requires different data during ongoing monitoring, especially for acute and chronic patients. (15.2.4) (15.2.5)

Nurses see it as a particular need for acute and chronic patients to have ongoing monitoring, as patients' condition often worsens due to lack of communication with them (15.2.2). Currently communication with patients remotely happens only by phone, for any quick referral in emergency cases. (15.2.4) (15.2.5) Based on interviews with nurses, it turns out that they especially miscommunication with patients and health staff about scheduling appointments with doctors, and also administratively, nurses face the problem of identifying medications and beds for patients under treatment (15.2.2). Whereas, during interviews with managers turns out that the main problem is the malfunction of a unique system between the primary, secondary and tertiary level in the Kosovo health system for managing health staff data, as well as data for treatment of patients on a monthly basis and annually (15.2.3).

Interviews with acute and chronic patients showed regular and frequent communication with the healthcare staff and also with other stakeholders is a problem. They find that lack of digital communication preventing them frequent consultations with their doctors about problems or even the occasional needs they have with other stakeholders such as pharmacies and insurance companies (15.2.3). For this reason, they see digital communication as a good 
option, they consider the current methods, such as face-to-face contact with the physician, to be troublesome for patients, especially for the acute and chronic patient group, since long waiting time is required, and also cost for treatment is high. The need for digital communication from patient groups is seen as a good opportunity, but they feel that family support is needed in specific cases, so they think they can become part of a digital remote

monitoring. While the group of healthy patients recognized the digital system enables them to become aware of routine checkup or to take preventive measures in the future (15.2.6).

\subsubsection{Proposed solution}

During the strategy implementation, it is important to investigate and understand the interested parties' requirements and their expected behavior in the healthcare system and translate them as functional user requirements, functional points of the system. Based on the demands of healthcare staff and patients we saw a great need for communication between stakeholders, especially medical and non-medical stakeholders. From an architectural design point of view, information system architecture plays a crucial role, especially in the context of different types of interaction where it involves the involvement of all stakeholders, which is also the main objective of an information system. The architecture proposal has three main pillars:

- Continuity of care: one or more healthcare providers provide services to a patient in a coordinated manner. This organizational principle focuses on time ties between various health services (with focus on medical history).

- Joint care: two or more healthcare providers collaborate providing services to a particular patient for ongoing diagnosis and/or treatment.

- Careful care: focuses on the timely and adequate transfer of activities and information, the responsibility for providing healthcare has been fully or partially transferred from one healthcare provider to another (focus on shared medical intervention).

In our technology architecture proposal the key role is played by genuine stakeholder communication, the architecture encompasses all stakeholders ranging from hospitals of all levels, pharmacies, outpatient services, insurance companies, health-data centers and finally 
patients. All the stakeholders have been offered intelligent communication solutions, utilizing the latest computer networking technologies, ranging from $4 \mathrm{G}$, mobile application links, healthcare dedicated links, VPN traffic secure, different communication protocols for each level of access, as well as access to IoT monitoring devices for patients, especially two target groups, acute and chronic patients using (IBM MQTT) messaging protocol for medical sensors.

The proposed network communication includes the Ministry of Health, which is the Core network data center, where the network starts with a full redundancy network at the central communication level and then links hospitals and other institutions with real-time communication opportunities using the ISP internet with a dedicated line to each institution. This proposed solution at the level of technology architecture gives creates secure communication and good internet speed, for all stakeholders. At the same time, this communication solution is a key prerequisite and basis for enabling communication of all stakeholders. They initially communicate over the Internet but at the same time, there is an opportunity to use different applications and data exchange platforms. In our proposal, the basic principle of data architecture is to provide information to all stakeholders and at the same time allow standardized information processing. Another design principle is the possibility to have unique information from different databases for all stakeholders. The Service-Oriented Architecture (SOA) solution enables different applications to communicate with different databases. Starting from communicating with the Civil Registration Database, which helps to have accurate information on patient from the state civil registry. This database contains trusted information concerning the patient's history allowing creation patient's profile, and the database accessible to all health institutions. The Healthcare Provider Database stores and maintains healthcare data generated during medical services, ranging from recordings, patient monitoring, to generated photographs. This database allows each healthcare staff to communicate and have a clear overview of the patient's prior treatment, and avoids such existing problems than misdiagnosis, inappropriate treatment, duplicating treatment.

Communication goes on between nurses and hospital managers using the Hospital Resources Database. The database contains data to track available rooms and beds, current 
patient numbers and the number of new patients being treated, helps efficiently coordinate and plan resources. The health institution, also other stakeholders, always need data on patients' health insurance payments. Therefore, the Healthcare Insurance Database stores patients' health insurance payments and expenditures.

Doctors, nurses and pharmacists need to communicate about prescription status and other information, (15.2.1), (15.2.2) this is enabled by communicating with the Prescription Center Database, which stores all prescriptions that are prescribed by physicians. The healthcare provider stores all records of the diagnosis, treatment, and medication prescribed for each patient separately and sends prescriptions regularly to the prescription center. The Prescription Center transfers prescriptions to the pharmacy database to check availability and in case of out of stock suggest substitution. This medication-oriented communication lowers the cost of patients, as pharmacists and physicians having the right information concerning the recommended therapies. The Pharmacy Database stores inventory information. The sales information recorded per patient allows precise reimbursement by sending the invoice to the patient's health insurance company.

The main goal of the health system is to provide information from secure data of different databases for making decision. However, each stakeholder has a different focus on using data to make the right decisions to improve health services. Using the Watson analytics system, doctors, hospital managers, and other stakeholders can create custom APIs for different purposes. A physician using a specific API can monitor patients in real-time for their health status generated by monitoring, and especially for preventive measures when a patient is alerted to their poor health. Hospital managers using the Watson analytics platform they can generate useful information to increase work efficiency, improve usage of human resources, inventory planning, process automation, and meet patient expectations. Government of Kosovo (Ministry of Health) as a stakeholder may have benefits also when plan future healthcare strategy.

The Application architecture allows access from all stakeholders, as it consists of various applications depending on the purpose of the stakeholders, including patient monitoring, data capture, report generation, data visualization. Specific modules and adequate privileges 
guaranteed for stakeholders, health or non-health institutions. Following patients' movement and medical episodes is the basic principle behind of the application architecture. Following this principle all relevant data is historically collected and provided selective access to the properly authenticated and authorized personnel. Realization also demands the standardized data flow among the various applications and platforms.

The Patient Portal also available for almost everybody including patients who allow the healthcare staff monitoring of their health, communication with pharmacies for drug status, as well as insurance companies to check healthcare payments. Emergency services that the patient can access using Mobile-based applications and web platforms to monitor his vital parameters are also part of the service portfolio. Monitoring can be specific according to the target groups. Services cover many areas of healthcare, including ECG monitoring, blood pressure, glucose, respiratory, external factors (humidity, wind, and temperature), drug taking rigorosity and physical activity through standardized sensors that can be directly linked through API to mobile application.

Whereas, the medical staff utilizing the communication through the Doctor's Portal enables continuous monitoring of the patients' health condition, thus enabling preventive measures for groups of acute and chronic patients, as well as checkups for non-patients. Medical staff can also communicate with pharmacies and emergency services using the Emergency services application, which enables healthcare staff to have information from all stakeholders in order for health services to be efficient, thus utilizing information from many parties that are directly linked to health services.

\subsubsection{Reflection}

In most stakeholder requirements and problems identified by direct interviews, we find a proposed architecture realistic at all three levels. Technology architecture fully supports communication, data architecture covers data sharing requirements from all stakeholders and in application architecture, and we see a good opportunity to provide information from all stakeholders ranging from healthcare staff, patients and health staff: pharmacies, insurance companies and the Ministry of Health. Based on expert opinions, the proposed architecture at the application level comply with the stakeholder requirements, only that the experts 
during the interviews recommend that access levels should be allocated to each user using the application (15.2.8).

Considering network infrastructure, computer-networking experts highlighted that real-time communication with the recommended topology is possible, but the cost and the way of encrypting traffic may create double load in some cases on access and during communication (15.2.9).

Interview with database experts showed, at the data level the communication design based on micro-services is acceptable and is an adequate choice (15.2.8). In addition, the Watson statistical system for generating data for specific parties is a good option, but they recommend the API should be professionally configured as it deals with many different databases and therefore requires professionalism in configuration (15.2.7), (15.2.8).

Looking at the perspective of stakeholder requirements on one hand, and proposal together with the expert recommendations on the other hand, we can say that the architecture proposal positively answers the research question.

\subsection{Scalability (RQ3)}

'Will and how the proposed eHealth system scalable and modularized enough to address the different technical and cultural readiness of various user group?"

\subsubsection{Problems and requirements}

During the interviews with target groups of both medical and non-medical staff they generally view my research as a good opportunity to solve problems and meet their demands for a better healthcare system. According to their opinion digitalization is the only way and solution to get out of current situation in which the health system is in Kosovo. While older patients and older healthcare staff see it as a challenge to adapt to a digital health ecosystem, they agreed ICT has affected every areas of their lives; it is more natural if they would be adapted to the digital health system (15.2.1) (15.2.4) (15.2.5).

During the interviews with patients, we see a positive acceptance in terms of monitoring equipment and various platforms for monitoring their health. They think that it will not be a problem to use health devices while monitoring their health, since everyone uses a smart 
communication device. Nevertheless, on the other hand, some of them think that monitoring over the long term can affect their mental health; some people in the interviewed groups think continuous monitoring is a mental obstacle (15.2.6). Most interviewees agree that digital monitoring would be welcome and necessary only when they are under medical treatment by healthcare staff (15.2.5), (15.2.6). In terms of data security and data protection most of them do not have much knowledge about data protection. However, they think that the security of the traditional version is not that reliable, because their paper data is in the hands of the medical staff and they never know who and how uses their data (15.2.6). Medicinal staff and most middle-aged and young healthcare staff see the use of electronic platforms for the treatment of patients as a good opportunity, but express the urgent need to train all staff before and during the use of the information system.

\subsubsection{Proposed solution}

For us it is very important to emphasize the evolvement of different architectures and their interactions in the ecosystem. The interoperability feature of the architecture is the basis for the proposed strategy providing undeniable proof that our architecture proposal has the potential to properly address customer requests. From an architecture design point of view the information system architecture bears a decisive role, especially in the context of different types of interoperability. In terms of adapting our architecture to the users, our architecture proposal largely meets most of the target and non-healthy user target groups and requirements, especially the web architecture based on the WEB and Mobile Platform. This platform allows for and adapts all end-users, which use electronic devices (PCs, mobile tablets, smartwatch) with minimal hardware performance. In addition, the applications' internal architecture is based on Services Orientation Architecture (SOA) and is adaptable to any changes adding future features in existing applications. The application architecture also meets the target group requirements for real-time monitoring, as through standardized sensors they can be directly linked through dedicated IoT protocol (MQQTT), and mobile application-specific API creation. At the data architecture, level it is adapted to communicate directly with all applications and is based on micro-services that helps make each database independent of other databases. Also allows upscaling in the future if one creates new 
database, and at the same time we can easily incorporate into the existing platform, only additional API configurations are required from existing applications to the new database.

The Watson analytics platform, where e-Health applications are running, can also easily be reconfigured for any platform or request coming from users through custom APIs depending on the target group of users. It is enough to create APIs in terms of analytics platform for the application and the various outbound databases and using artificial intelligence to make decisions based on the data received from the applications in real time as well as on the existing data from different already existing databases. This platform adds great value and importance to the information system and it is adaptable to any applied technology.

The technology architecture is largely scalable since the hardware and software levels are easily adaptable. This is due to the fact that at hardware level of computer networking to each devices additional communication modules can be added, depending on system requirements. At configuration level, it is always permissible to configure additional services on existing equipment and this allows adaptation to any situation and requirement depending on technological changes or performance. At the level of user access as they use $4 \mathrm{G}$ mobile networks and mobile devices can easily adapt to changes in other frequency bands if this technology is required. Whereas, at the level of in-censor communication with mobile devices, the MQQTT protocol enables most IoT sensors to be connected with minimum energy efficiency and finally, it is an IBM standardized protocol.

Virtualization of data services and applications has been recommended in the datacenter, so through virtualization we can improve service performance as well as providing with convenience for speeding up data processing and expanding data storage space. At the security level, architecture from the simple user to the data center we recommended optimal security by encrypting traffic from accessing web applications and mobile platforms using the HTTPS protocol as the first level of security. In distribution networks, we recommended a VPN tunnel for encrypting traffic from hospital centers to datacenter. This recommendation stands for the data level and especially for datacenter within servers using AllwaysON SQL technology solution, which provides physical and logical security of communication data. The use and adaptation of the digital system by all stakeholders that will utilize health 
services is planned as a specific phase in the implementation of the strategy. In our proposal, staff training and awareness raising for the use of technological equipment in health services is planned, especially target groups in this research. Awareness and training methods are planned to be using direct training, awareness campaigns, through TV, social networks using age-specific filters, as well as seminars (9.3.1).

\subsubsection{Reflection}

At the application level, experts think that the recommended web / mobile platform and solution, based on SOA architecture think it's the right choice, but they think communication protocols need to be chosen carefully, because data representation on the applications is easy to do, but communication between them is a challenge which need to be treated carefully. (15.2.7), (15.2.11) Experts also recommend that defragmenting roles at the application level plays an important role (15.2.8). While, regarding application-level communication performance, experts think that the solution - offered by the proposed architecture in terms of service virtualization - is adequate, the only suggestion is that applications and data should be hardware-separated as this shared solution enhances the performance of applications (15.2.8). At the communication level the experts have a positive opinion as they see an adequate solution ranging from end users to datacenters. Whereas, experts see double encryption of traffic as a challenge, as it requires high performance of networking equipment and especially core level (15.2.9), (15.2.10). At the information system level, experts generally have a positive opinion about the solutions offered, but it remains to be seen once the proposal is implemented (15.2.11).

Given the user requirements and problems, expert recommendations, and the proposed architecture we find a strong link between user requirements and the recommended architecture proposal. The proposed architecture is largely scalable and can easily be adapted to changes depending on user requirements, especially changes and requirements for adding new features and enhancing performance in line with the evolution of new technologies. Therefore, based on the requirements of certain groups and on the other hand based on the architecture proposal, as well as the recommendations and opinions of experts, we see a realistic solution to the requirements regarding scalability and modularity user groups. 


\section{Conclusion and Future Direction}

Health information is important for monitoring and improving people's health. The results of the study prove that in Kosovo the health system still has problems in providing health services, in both professional and technical aspects. This is due to the lack of a unique electronic health system that would monitor patients' health status and possess important information about them, especially acute and chronic ones. Lack of health information also affects the quality of health services provided. Improvement of the health system in Kosovo is achieved when the information system addresses national demands and problems. The progress of healthcare delivery relies on good decision-making in the implementation of the long-term strategy involving all stakeholders in the health system. Best practices, challenges and lessons learned in health system implementation in developed countries are guidelines

for developing information systems in developing countries, such as Kosovo. The success and failure of any strategy in health systems depends on good planning ranging from vision, implementation steps, technology selection, and involvement of all stakeholders in implementation planning.

The health information system carries a heavy burden on the health information it possesses. Therefore, the selection of technologies for the implementation of the digital system requires long-term solutions at three levels of architecture ranging from application architecture that receives information, data architecture that creates value from the accumulated data, and technology architecture that supports the two aforementioned architectures in their efficient and safe operation. A realistic architecture should initially provide information to all stakeholders and at the same time allow each other to process the information independently to a single standard. It is very important to emphasize the evolution of different architectures and their interactions in the ecosystem, especially in the context of different types of interaction. Therefore, the selection of appropriate technologies directly affects the ability to scale the architecture and adapt it to the requirements of users and the health system. Handling and identifying the requirements of all stakeholders providing health services is essential, and incorporating them into the digital system is vital to the functioning of the health information system. The selection and interconnection of Web and Mobile platforms for access to information by different stakeholders plays an important role in the 
communication / improvement and efficiency of health services. Communication using technology devices and digital platforms between healthcare staff and patients, on one hand improves the health of patients, and increases the progressivity of work by the healthcare staff on the other hand. Problem and challenge remains the adaptation and training of deprived ages to be part of the exploitation of the digital health system.

The results of this research highlight that digitalization of the health system is indispensable for improving the current state of affairs in both health and administrative terms, always taking into account user requirements and experts' opinion on the chosen technology. By using ICT in healthcare benefits many stakeholders, ranging from the population, health care providers, the state, and all other stakeholders that are part of the health ecosystem.

\section{Future direction}

eHealth information systems are advancing very fast as information technology is advancing every day. As performance and data quality increase, so does the demand for new technologies. In health information systems this is evident with the incorporation of real-time monitoring for patients, which has at the same time increased the volume of data requiring greater management and security during communicating between different platforms. Cloudbased services the inclusion of IoT devices in healthcare, sends a preparation for a new phase of implementation of cloud-based services as processing large volumes of data requires more hardware resources and much more maintenance difficult.

The only solution that can meet the challenges is cloud services, but the security of health data remains a challenge. Also, with the introduction of the $5 \mathrm{G}$ network, the demand for mobile applications for monitoring patients and other services will increase, but the challenge of maintaining the energy of the devices as they will withstand 5G communication. Also security in $5 \mathrm{G}$ communications still remains controversial as to how it will be handled. Implementing block chain technology in eHealth systems is seen as a future that will enhance the security aspect of communication between stakeholders, but it is still worth rethinking how successful block chain technology will be in banking systems. The future of digital systems in medicine it will be a top priority across the globe as it is directly linked to the health of humankind all over the world. 


\section{References}

Abdusamadovich, L. M., \& Sciences, N. (2013). Global Standards and Local Health Information System Applications: Understanding their interplay in the context of Tajikistan, (June).

Adebesin, F. (2013a). A Review of Interoperability Standards in E-health and Imperatives for their Adoption in Africa. Applied Computing, 50(50), 55-72. https://doi.org/10.18489/sacj.v50i1.176

Adebesin, F. (2013b). Barriers \& challenges to the adoption of E-Health standards in Africa. Proceedings of Health Informatics South Africa 2013 (HISA 2013) Conference. Retrieved from http://researchspace.csir.co.za/dspace/handle/10204/6910

Al-Janabi, S. A.-S. (2017). Survey of main challenges (security and privacy) in wireless body area networks for healthcare applications. Egyptian Informatics Journal, Volume 18, Issue 2, Pages 113-122. https://doi.org/10.1016/j.eij.2016.11.001

Al Ameen, M., \& Kwak, K. (2011). Social issue in wireless sensor networks for healthcare perspective. The International Arab Journal of Information Technology, 8(1), 15-21. https://doi.org/10.1.1.172.5467

Al Ameen, M., Liu, J., \& Kwak, K. (2012). Security and privacy issues in wireless sensor networks for healthcare applications. Journal of Medical Systems, 36(1), 93-101. https://doi.org/10.1007/s10916-010-9449-4

Albahri, O. (2018). Systematic Review of Real-time Remote Health Monitoring System in Triage and Priority-Based Sensor Technology. Journal of Medical Systems, 42(5).

Alemdar, H., \& Ersoy, C. (2010). Wireless sensor networks for healthcare: A survey. Computer Networks, 54(15), 2688-2710. https://doi.org/10.1016/j.comnet.2010.05.003

Alves, R. S., Marquezan, C. C., \& Granville, L. Z. (2015). Experiences in the implementation of an SNMP-based high performance cluster management system. Proceedings. ISCC 2004. Ninth International Symposium on Computers And Communications (IEEE Cat. No.04TH8769), (April), 1136-1141. https://doi.org/10.1109/ISCC.2004.1358692

Aminian, M. (2013). A Hospital Healthcare Monitoring System Using Wireless Sensor Networks. Journal of Health \& Medical Informatics, 04(02), 4-9. https://doi.org/10.4172/2157-7420.1000121 
Anker, S. D., Koehler, F., \& Abraham, W. T. (2011). Telemedicine and remote management of patients with heart failure. The Lancet, 378(9792), 731-739. https://doi.org/10.1016/S0140-6736(11)61229-4

Arak, P., \& Wójcik, A. (2017). Transforming eHealth into a political and economic advantage. Digital Single Market, Report and Studies. Retrieved from https://bit.ly/2tBwV0C

Atanasovski, B., Bogdanović, M., Velinov, G., Stoimenov, L., \& Sahpaski, D. (2017). Transforming an Enterprise E-Health System from Process Oriented to Model Driven Architecture, (July 2018), 159-162.

BCG. (2012). The Socio-Economic Impact of Mobile Health, (April), 1-49.

Benharref, A., \& Serhani, M. A. (2014). Novel cloud and SOA-based framework for E-health monitoring using wireless biosensors. IEEE Journal of Biomedical and Health Informatics, 18(1), 46-55. https://doi.org/10.1109/JBHI.2013.2262659

Bhavnani, S. P., Narula, J., \& Sengupta, P. P. (2016). Mobile technology and the digitization of healthcare. European Heart Journal, 37(18), 1428-1438. https://doi.org/10.1093/eurheartj/ehv770

Capolongo, S. (2015). Improving Sustainability During Hospital Design and Operation, (February), 10-22. https://doi.org/10.1007/978-3-319-14036-0

Carrasqueiro, S. (2017). The establishment of a platform for the sharing of national eHealth strategies. $\quad$ Portugal. Retrieved from https://ec.europa.eu/health/sites/health/files/ehealth/docs/ev_20170509_co14_en.pdf

CAST. (2013). Telehealth and remote patient monitoring (rpm) - Provider case studies 2013. LeadingAge CAST Report.

Cerdan, J., Catalan-Matamoros, D., \& Berg, S. W. (2017). Online communication in a rehabilitation setting: Experiences of patients with chronic conditions using a web portal in Denmark. Patient Education and Counseling, 100(12), 2283-2289. https://doi.org/10.1016/j.pec.2017.06.023

Chatterjee, S., Das, A. K., \& Sing, J. K. (2014). A novel and efficient user access control scheme for wireless body area sensor networks. Journal of King Saud University Computer and Information Sciences, 26(2), 181-201. https://doi.org/10.1016/j.jksuci.2013.10.007 
Chaudhry, S., Mattera, J., Curtis, J., Spertus, J., Herrin, J., Lin, Z., ... Krumholz, M. D. (2011). Telemonitoring in Patients with Heart Failure. N Engl J Med., 363(24), 23012309. https://doi.org/10.1056/NEJMoa1010029.Telemonitoring

Chen, M., Gonzalez, S., Vasilakos, A., Cao, H., \& Leung, V. C. M. (2011). Body area networks: A survey. Mobile Networks and Applications, 16(2), 171-193. https://doi.org/10.1007/s11036-010-0260-8

Chipman, A. (2016). Modernising health systems in the Balkans: Uneven progress. Retrieved from

https://www.eiuperspectives.economist.com/sites/default/files/Modernisinghealthsyste msintheBalkans_0.pdf

Chooruang, K., \& Mangkalakeeree, P. (2016). Wireless Heart Rate Monitoring System Using MQTT. Procedia Computer Science, 86(March), 160-163. https://doi.org/10.1016/j.procs.2016.05.045

Christensen, J. K. B. (2018). The emergence and unfolding of telemonitoring practices in different healthcare organizations. International Journal of Environmental Research and Public Health, 15(1). https://doi.org/10.3390/ijerph15010061

Christodoulou, E., Dunbar, A., Gáspár, P., Jaksa, R., \& Krapez, K. (2008). The Development of eHealth in an Enlarged EU SYNTHESIS REPORT. Retrieved from http://ftp.jrc.es/EURdoc/JRC43277.pdf

Danko, D., \& Petrova, G. (2014). Health technology assessment in the balkans: Opportunities for a balanced drug assessment system. Biotechnology and Biotechnological Equipment, 28(6), 1181-1189. https://doi.org/10.1080/13102818.2014.978636

De Lyon, A. T. C., Neville, R. D., \& Armour, K. M. (2017). The Role of Fitness Professionals in Public Health: A Review of the Literature. Quest, 69(3), 313-330. https://doi.org/10.1080/00336297.2016.1224193

DeLyon, A., Neville, R., \& Armour, K. (2017). The Role of Fitness Professionals in Public Health: A Review of the Literature. Quest, 69(3), 313-330. https://doi.org/10.1080/00336297.2016.1224193

Ellingsen, G., Christensen, B., \& Silsand, L. (2014). Developing Large-scale Electronic Patient Records Conforming to the openEHR Architecture. Procedia Technology, 16(2212), 1281-1286. https://doi.org/10.1016/j.protcy.2014.10.144 
European Commission\&OECD. (2016). Health at a Glance: Europe 2016 - State of Health in the EU Cycle. OECD Publishing. https://doi.org/10.1787/9789264012639-en

European Commission\&OECD. (2018). Health at a Glance: Europe 2018 STATE OF HEALTH IN THE EU CYCLE. https://doi.org/10.1787/health_glance_eur-2018-en

European Commission. (2018). THE CATHOLIC CHURCH IN THE EUROPEAN UNION WESTERN BALKANS ' EUROPEAN INTEGRATION - “ A PROMISE OF PEACE AND A SOURCE OF DEVELOPMENT '?, 1-8.

Fagerberg, J., \& Kurkinen, L. (2012). mHealth and Home Monitoring. M2M Research Series, 2-5. Retrieved from http://www.berginsight.com/ReportPDF/ProductSheet/bimhealth5-ps.pdf

Fairburn, C. G., \& Patel, V. (2017). The impact of digital technology on psychological treatments and their dissemination. Behaviour Research and Therapy, 88, 19-25. https://doi.org/10.1016/j.brat.2016.08.012

Fehr, A. (2018). European Core Health Indicators. Archives of Public Health, 76(1), 52. https://doi.org/10.1186/s13690-018-0298-9

Fehr, A., Tijhuis, M. J., Hense, S., Urbanski, D., Achterberg, P., \& Ziese, T. (2018a). European Core Health Indicators - status and perspectives. Archives of Public Health, 76(1), 52. https://doi.org/10.1186/s13690-018-0298-9

Fehr, A., Tijhuis, M. J., Hense, S., Urbanski, D., Achterberg, P., \& Ziese, T. (2018b). European Core Health Indicators - status and perspectives. Archives of Public Health, 76(1), 52. https://doi.org/10.1186/s13690-018-0298-9

Gjorgjev, D., Bacanovic, A., Cicevalieva, S., Sulevski, Z., \& Grosse-Tebbe, S. (2006). The former Yugoslav Republic of Macedonia: Health system review. Health Systems in Transition, 8(2). Retrieved from http://www.euro.who.int/_data/assets/pdf_file/0005/96413/E89275.pdf

Greenspun, H., \& Coughlin, S. (2012). mHealth in an mWorld - How Mobile Technology is Transforming Healthcare. Deloitte Center for Health Solutions, 1-21.

Grieco, L. A., Rizzo, A., Colucci, S., Sicari, S., Piro, G., Di Paola, D., \& Boggia, G. (2014). IoT-aided robotics applications: Technological implications, target domains and open issues. Computer Communications, $\quad$ 54, https://doi.org/10.1016/j.comcom.2014.07.013 
Grosios, K., Gahan, P. B., \& Burbidge, J. (2010). Overview of healthcare in the UK. EPMA Journal, 1(4), 529-534. https://doi.org/10.1007/s13167-010-0050-1

Guedria, W., Lamine, E., Pingaud, H., Guedria, W., Lamine, E., \& Pingaud, H. (2015). Health systems interoperability : analysis and comparison To cite this version : HAL Id : hal-01166694.

Hammond, W. (2010). EU eHealth Interoperability Roadmap. Studies in Health Technology and Informatics, 134(December), 245-253. Retrieved from http://www.ncbi.nlm.nih.gov/pubmed/22894051\%5Cnhttp://books.google.com/books? $\mathrm{hl}=\mathrm{en} \& \mathrm{lr}=\& \mathrm{id}=\mathrm{KtSZC} 8 \mathrm{C}$ a0wC\&oi=fnd\&pg=PA245\&dq=eHealth+Interoperability\&ots=uMAROWo89f\&sig=j 2JtH_ZNzUCAEmVLGpGTSk2HK60

Hassanalieragh, M., Page, A., Soyata, T., Sharma, G., Aktas, M., Mateos, G., ... Andreescu, S. (2015). Health Monitoring and Management Using Internet-of-Things (IoT) Sensing with Cloud-Based Processing: Opportunities and Challenges. In Proceedings - 2015 IEEE International Conference on Services Computing, SCC 2015. https://doi.org/10.1109/SCC.2015.47

HMSO. (2009). The National Programme for IT in the NHS: Progress since 2006 - Second Report of Session 2008-09, (May), 54.

Hodzic, S. I. (2017). A Situational Analysis of the Western Balkans - 2017. Brussels, Beligium. Retrieved from http://www.eupatient.eu/globalassets/library/toolkits/situational_analysis_balkans_2017.pdf

Imasheva, A., \& Seiter, A. (2008). The pharmaceutical sector of the Western Balkan countries (Inglés). Retrieved from http://documents.worldbank.org/curated/en/307671468012340807/pdf/428270WP01N O0P11sinWesternBalkansDP.pdf

Jovanov, E., \& Milenkovic, A. (2011). Body area networks for ubiquitous healthcare applications: Opportunities and challenges. Journal of Medical Systems, 35(5), 12451254. https://doi.org/10.1007/s10916-011-9661-x

Jovanov, E., Milenkovic, A., Otto, C., \& De Groen, P. C. (2005). A wireless body area network of intelligent motion sensors for computer assisted physical rehabilitation. Journal of NeuroEngineering and Rehabilitation, 2, 1-10. https://doi.org/10.1186/1743-0003-2-6

KAS. (2013). Seria 5 : Statistikat Sociale Statistikat e Arsimit 2012-2013. Retrieved from 
http://ask.rks-gov.net/arsimi/publikimet/doc_view/1120-statistikat-e-arsimit-20122013?tmpl=component\&format=raw

KAS. (2017a). Seria 4 : Statistikat Sociale Rezultatet e Anketës së Përdorimit të Teknologjisë Informative dhe Komunikimit.

KAS. (2017b). Seria 5: Statistikat Sociale Statistikat e Shëndetësisë (Health Statistics). Kosovo Agency of Statistics - KAS. Retrieved from http://ask.rks-gov.net/sq/agjencia-estatistikave-te-kosoves/add-news/statistikat-e-shendetesise-2015

KAS. (2018). Statistikat Sociale Statistikat e Arsimit 2012-2013. Prishtina, Kosovo. Retrieved from http://data.rks-gov.net/dataset/59273966-78a9-4ee6-a2783189fa6309b0/resource/1b97aee9-6a78-4f65-abdad2004e47c30e/download/statistikat-e-arsimit-2013-2014.pdf

Kim, Y. K., Wang, H., \& Mahmud, M. S. (2016). Wearable body sensor network for health care applications. In Smart Textiles and Their Applications. https://doi.org/10.1016/B978-0-08-100574-3.00009-6

Krishnamurthy, R. S. (2012). Health Data Standardisation and Interoperability. Geneva,, Switzerland. Retrieved from https://www.who.int/ehealth/WHO_Forum_on_HDSI_Report.pdf

Krishnamurthy, R. S. (2013). eHealth Standardization and Interoperability and related eHealth activities at WHO Headquarters. Geneva, , Switzerland: Joint IEEE, ITU and WHO session on e-Health -.

Kumar, P., \& Lee, H.-J. (2011). Security Issues in Healthcare Applications Using Wireless Medical Sensor Networks: A Survey. Sensors, 12(12), 55-91. https://doi.org/10.3390/s120100055

Lai, T., Habicht, T., Kahur, K., Reinap, M., Kiivet, R., \& van Ginneken, E. (2013). Estonia: health system review. Health Systems in Transition, 15(6), 1-196.

Langenhove, P. van, Decreus, K., Rogala, A., Olyslaegers, T., \& Whitehouse, D. (2013). eHealth European Interoperability Framework: Vision on eHealth EIF. Study. https://doi.org/10.2759/15839

Lee, S., Ghasemzadeh, H., \& Mortazavi, B. (2013). Remote Patient Monitoring: What Impact Can Data Analytics Have on Cost? ACM Wireless Health, Baltimore, MD, USA. Retrieved from http://cs.ucla.edu/ silee/pub/acm_wh_2013.pdf 
Lloret, J., Parra, L., Taha, M., \& Tomás, J. (2017). An architecture and protocol for smart continuous eHealth monitoring using 5G. Computer Networks, 129, 340-351. https://doi.org/10.1016/j.comnet.2017.05.018

Lorenz, A., \& Oppermann, R. (2009). Mobile health monitoring for the elderly: Designing for diversity. Pervasive and Mobile Computing, 5(5), 478-495. https://doi.org/10.1016/j.pmcj.2008.09.010

Maccallum, R. C., \& Austin, J. T. (2000). Applications of Smartphones for Ubiquitious Health Monitoring and Wellbeing Management. Annual Review of Psychology, 1, 201226. https://doi.org/10.1146/annurev.psych.51.1.201

Marcos, M., Maldonado, J. A., Martínez-Salvador, B., Boscá, D., \& Robles, M. (2013). Interoperability of clinical decision-support systems and electronic health records using archetypes: A case study in clinical trial eligibility. Journal of Biomedical Informatics, 46(4), 676-689. https://doi.org/10.1016/j.jbi.2013.05.004

Meingast, M., Roosta, T., \& Sastry, S. (2006). Security and privacy issues with health care information technology. Conference Proceedings : ... Annual International Conference of the IEEE Engineering in Medicine and Biology Society. IEEE Engineering in Medicine and Biology Society. Conference, 1, 5453-5458. https://doi.org/10.1109/IEMBS.2006.260060

Milenković, D., Milenković, M. J., Vujin, V., Aleksić, A., \& Radojičić, Z. (2012). Electronic health system - Development and implementation into the health system of the Republic of Serbia [Elektronski zdravstveni sistem - Razvoj i uvod\{stroke\}enje u zdravstveni sistem Republike Srbije]. Vojnosanitetski Pregled, 69(10), 880-890. https://doi.org/10.2298/VSP101125021M

Milito, R., Morrow, M., Lake, D., \& Vargheese, R. (2014). Internet of Things: Architectural Framework for eHealth Security. Journal of ICT Standardization, 1(3), 301-328. https://doi.org/10.13052/jicts2245-800x.133

MoH. (2010a). Health Sector Strategy 2010 -2016, (May 2009).

MoH. (2010b). Kosovo Health Strategy. Prishtina. Retrieved from https://msh.rksgov.net/wp-content/uploads/2013/11/Strategjia-Sektoriale-e-Shendetesise-20102014.pdf

MoH. (2010c). STRATEGJIA PËR SISTEMIN E INFORMIMIT SHËNDETËSOR NË KOSOVË. Prishtina, Kosovo. Retrieved from http://www.kryeministriks.net/repository/docs/Strategjia-sektoriale-e-shendetesise-final-nentor-2016- 


\section{ALB_.pdf}

Mountford, N., \& Kessie, T. (2016). CONNECTED HEALTH IN EUROPE. Dublin.

Mshali, H. (2018). A survey on health monitoring systems for health smart homes. International Journal of Industrial Ergonomics, 66, 26-56. https://doi.org/10.1016/J.ERGON.2018.02.002

Mshali, H., Lemlouma, T., \& Magoni, D. (2018). Adaptive monitoring system for e-health smart homes. Pervasive and Mobile Computing, 43, 1-19. https://doi.org/10.1016/j.pmcj.2017.11.001

Myers, S., Grant, R. W., Lugn, N. E., Holbert, B., \& Kvedar, J. C. (2006). Impact of HomeBased Monitoring on the Care of Patients with Congestive Heart Failure. Home Health Care Management \& Practice, 18(6), 444-451. https://doi.org/10.1177/1084822306289991

Nadeem, A., Hussain, M. A., Owais, O., Salam, A., Iqbal, S., \& Ahsan, K. (2015). Application specific study, analysis and classification of body area wireless sensor network applications. Computer Networks, 83, 363-380. https://doi.org/10.1016/J.COMNET.2015.03.002

National Audit. (2008). HC484 The National Programme for IT in the NHS : Project Progress Reports, (May).

National Audit. (2017a). EFIKASITETI DHE EFEKTIVITETI NË IMPLEMENTIMIN E SISTEMIT INFORMATIV UNIK TË INTEGRUAR SHËNDETËSOR (21x;22.0.102010/17-08). Prishtina. Retrieved from http://www.zka-rks.org/wpcontent/uploads/2017/12/Raporti_auditimit_-SISH_shq.pdf

National Audit. (2017b). RAPORT I AUDITIMIT SISTEMIT INFORMATIV UNIK TË INTEGRUAR SHËNDETËSOR Auditim i performancës, (i). Retrieved from http://www.zka-rks.org/wp-content/uploads/2017/12/Raporti_auditimit_SISH_shq.pdf

Newman, M., \& McMahon, T. (2011). Potential Cost Savings from Expansion of Telehealth Center for Connected Health Policy. Blue Sky Consulting Group.

OECD/EU. (2016). Health at a Glance: Europe 2016 - State of Health in the EU Cycle. OECD Publishing. https://doi.org/10.1787/9789264012639-en 
Oleshchuk, V., \& Fensli, R. (2011). Remote patient monitoring within a future 5G infrastructure. Wireless Personal Communications, 57(3), 431-439. https://doi.org/10.1007/s11277-010-0078-5

Pahl, C., Zare, M., Nilashi, M., de Faria Borges, M. A., Weingaertner, D., Detschew, V., ... Ibrahim, O. (2015). Role of OpenEHR as an open source solution for the regional modelling of patient data in obstetrics. Journal of Biomedical Informatics, 55(2015), 174-187. https://doi.org/10.1016/j.jbi.2015.04.004

Pantelopoulos, A., \& Bourbakis, N. G. (2010). A survey on wearable sensor-based systems for health monitoring and prognosis. IEEE Transactions on Systems, Man and Cybernetics Part C: Applications and Reviews, 40(1), 1-12. https://doi.org/10.1109/TSMCC.2009.2032660

Pashazadeh, A., \& Navimipour, N. J. (2018). Big data handling mechanisms in the healthcare applications: A comprehensive and systematic literature review. Journal of Biomedical Informatics, 82(November 2017), 47-62. https://doi.org/10.1016/j.jbi.2018.03.014

Patel, S., Park, H., Bonato, P., Chan, L., \& Rodgers, M. (2012). A review of wearable sensors and systems with application in rehabilitation. Journal of NeuroEngineering and Rehabilitation, 9(1), 21. https://doi.org/10.1186/1743-0003-9-21

Pedace, C., Rosa, A., Francesconi, P., Acampora, A., Ricciardi, W., \& Damiani, G. (2017). [Governance in a project addressing care of disabled elderly persons within the regional healthcare system of Tuscany, Italy]. Igiene e Sanita Pubblica.

Peterson, C., Hamilton, C., \& Hasvold, P. (2016). From Innovation to implementation. World Health Organization, 1-12. https://doi.org/10.1016/j.jacc.2014.10.008

Piha, T. (2013). mHealth and Health Policies. In EHF Gastein.

Piha, T. (2015). The EU eHealth Strategy Connecting Member States. In 2nd National eHealth Summit. Carton House, Kildare. Retrieved from https://docplayer.net/22216626-The-eu-ehealth-strategy-connecting-memberstates.html

Piho, G., Tepandi, J., Thompson, D., Woerner, A., \& Parman, M. (2015). Business archetypes and archetype patterns from the HL7 RIM and openEHR RM perspectives: Towards interoperability and evolution of healthcare models and software systems. Procedia Computer Science, 63, 553-560. https://doi.org/10.1016/j.procs.2015.08.384 
Rahim, A., Kong, X., Xia, F., Ning, Z., Ullah, N., Wang, J., \& Das, S. K. (2018). Vehicular Social Networks: A survey. Pervasive and Mobile Computing, 43, 96-113. https://doi.org/10.1016/j.pmcj.2017.12.004

Rahmani, A. M., Gia, T. N., Negash, B., Anzanpour, A., Azimi, I., Jiang, M., \& Liljeberg, P. (2018). Exploiting smart e-Health gateways at the edge of healthcare Internet-ofThings: A fog computing approach. Future Generation Computer Systems, 78, 641658. https://doi.org/10.1016/j.future.2017.02.014

Raporti i auditimit për pasqyrat financiare vjetore të ministrisë së shëndetësisë për vitin e përfunduar më 31 dhjetor 2017. (2018), (i), 1-45.

Roland Berger. (2016). Digital and disrupted: All change for healthcare. Think Act, 19. Retrieved from www.rolandberger.com

Rolim, C. O., Koch, F. L., Westphall, C. B., Werner, J., Fracalossi, A., \& Salvador, G. S. (2010). A Cloud Computing Solution for Patient's Data Collection in Health Care Institutions. 2010 Second International Conference on EHealth, Telemedicine, and Social Medicine, (i), 95-99. https://doi.org/10.1109/eTELEMED.2010.19

Rosa, P. (2011). State of the art of international standards in Telemedicine and, (July).

Saleem, K., Derhab, A., Al-Muhtadi, J., \& Shahzad, B. (2015). Human-oriented design of secure Machine-to-Machine communication system for e-Healthcare society. Computers in Human Behavior, 51, 977-985. https://doi.org/10.1016/j.chb.2014.10.010

Santos, J., Rodrigues, J. J. P. C., Silva, B. M. C., Casal, J., Saleem, K., \& Denisov, V. (2016). An IoT-based mobile gateway for intelligent personal assistants on mobile health environments. Journal of Network and Computer Applications, 71, 194-204. https://doi.org/10.1016/j.jnca.2016.03.014

Shen, J., Gui, Z., Ji, S., Shen, J., Tan, H., \& Tang, Y. (2018). Cloud-aided lightweight certificateless authentication protocol with anonymity for wireless body area networks. Journal of Network and Computer Applications, 106. https://doi.org/10.1016/j.jnca.2018.01.003

Smaradottir, B., Gerdes, M., Martinez, S., \& Fensli, R. (2015). The EU-project United4Health: User-Centred Design and Evaluation of a Collaborative Information System for a Norwegian Telehealth Service. Studies in Health Technology and Informatics, 216(August), 892. https://doi.org/10.3233/978-1-61499-564-7-892 
Song, E., Sarver, H., Aranki, D., Bajcsy, R., \& Javey, A. (2016). A Telemonitoring Solution to Long-Distance Running Coaching.

Sopjani, I. (2016). Infermierët : Një forcë për ndryshim Përmirësimi i reziliencës së sistemeve shëndetësore.

Stachura, M., \& Khasanshina, E. (2007). Telehomecare and Remote Monitoring: An Outcomes Overview. Augusta Georgia Center for Telehealth Medical College of Georgia, 2892-2902. Retrieved from http://scholar.google.com/scholar?hl=en\&btnG=Search\&q=intitle:Telehomecare+and +Remote+Monitoring+:+An+Outcomes+Overview\#0

Stamate, C., Magoulas, G. D., Kueppers, S., Nomikou, E., Daskalopoulos, I., Jha, A., ... Roussos, G. (2018). The cloudUPDRS app: A medical device for the clinical assessment of Parkinson's Disease. Pervasive and Mobile Computing, 43, 146-166. https://doi.org/10.1016/j.pmcj.2017.12.005

Stuart, M., \& Weinrich, M. (2001). Home- and community-based long-term care: Lessons from Denmark. Gerontologist, 41(4), 474-480. https://doi.org/10.1093/geront/41.4.474

Stubbs, P. (2009). Social Protection and Social Inclusion in the Western Balkans: A Synthesis Report, (January).

Taylor, K. (2015). Connected health - How digital technology is transforming health and social care. Deloitte Centre for Healthcare Solutions, 40. https://doi.org/10.1016/j.cell.2007.03.014

Toçi, E. (2015). Health literacy in the Western Balkans The example of Albania and Kosovo. Universitaire Pers Maastricht.

Tresp, V., Marc Overhage, J., Bundschus, M., Rabizadeh, S., Fasching, P. A., \& Yu, S. (2016). Going Digital: A Survey on Digitalization and Large-Scale Data Analytics in Healthcare. Proceedings of the IEEE, 104(11), 2180-2206. https://doi.org/10.1109/JPROC.2016.2615052

Velinov, G., Jakimovski, B., Lesovski, D., Ivanova Panova, D., Frtunik, D., \& KonPopovska, M. (2015). EHR System MojTermin: Implementation and Initial Data Analysis. Studies in Health Technology and Informatics, 210, 872-876. https://doi.org/10.3233/978-1-61499-512-8-872

Vučetić, M., Uzelac, A., \& Gligorić, N. (2011). E-health transformation model in Serbia: 
Design, architecture and developing. Proceedings - 2011 International Conference on Cyber-Enabled Distributed Computing and Knowledge Discovery, CyberC 2011, (October), 566-573. https://doi.org/10.1109/CyberC.2011.96

Watzlaf, V. J. M., Moeini, S., Matusow, L., \& Firouzan, P. (2011). VOIP for Telerehabilitation: A Risk Analysis for Privacy, Security and HIPAA Compliance: Part II. International Journal of Telerehabilitation, 3(1), 3-10. https://doi.org/10.5195/ijt.2011.6070

Wetzels, M., Ayoola, I., Bogers, S., Peters, P., Chen, W., \& Feijs, L. (2018). Consume: A privacy-preserving authorisation and authentication service for connecting with health and wellbeing APIs. Pervasive and Mobile Computing, 43, 20-26. https://doi.org/10.1016/j.pmcj.2017.11.002

WHO/EU. (2016). FROM INNOVATION TO IMPLEMENTATION-eHealth in the WHO European Region. Copenhagen, Danimark: WHO Regional Office for Europe to: Publications WHO Regional Office for Europe. https://doi.org/10.1016/j.jacc.2014.10.008

WHO/ITU. (2012). National eHealth Strategy Toolkit. World Health Assembly Resolution and ITU World Telecom Development Conference Resolution, 1-2. https://doi.org/978 9241548465

WHO. (2006). Medical Records Manual : A Guide for Developing Countries. WHO Library Cataloguing in Publication Data, Western Pacific Region, 1-126. https://doi.org/10.2147/CLEP.S23690

WHO. (2011). mHealth: new horizons for health through mobile technologies: second global survey on eHealth. Global Observatory for EHealth Series, 3(June), 103. https://doi.org/10.4258/hir.2012.18.3.231

WHO. (2012). National eHealth Strategy Toolkit. World Health Assembly Resolution and ITU World Telecom Development Conference Resolution. https://doi.org/978 924 1548465

WHO. (2015). Core Health Indicators in the WHO European Region, 10.

WHO. (2016a). eHealth in the WHO European Region, 1-100. https://doi.org/10.1016/j.jacc.2014.10.008

WHO. (2016b). From Innovation to Implementation - eHealth in the WHO European Region. 
Innovation, 1-12. https://doi.org/10.1016/j.jacc.2014.10.008

Woodbridge, J., \& Tu, M. K. (2011). NIH Public Access, 35(5), 1165-1179. https://doi.org/10.1007/s10916-011-9733-y.A

World Health Organization. (2016). From Innovation to Implementation - eHealth in the WHO European Region. Innovation, 1-12. https://doi.org/10.1016/j.jacc.2014.10.008

Yaseen, M., Saleem, K., Orgun, M. A., Derhab, A., Abbas, H., Al-Muhtadi, J., ... Rashid, I. (2018). Secure sensors data acquisition and communication protection in eHealthcare: Review on the state of the art. Telematics and Informatics, 35(4), 702-726. https://doi.org/10.1016/j.tele.2017.08.005

Yuan, B., \& Li, J. (2019). The Policy Effect of the General Data Protection Regulation (GDPR) on the Digital Public Health Sector in the European Union: An Empirical Investigation. International Journal of Environmental Research and Public Health, 16(6), 1070. https://doi.org/10.3390/ijerph16061070

Ziefle, M. (2011). Technologies and Applications for Assisted Living: Challenges and Solutions. Achen,Germany: Medical Information Science Reference (an imprint of IGI Global). Retrieved from http://www.comm.rwthaachen.de/files/igiglobal_alle_2011.pdf 


\section{Publication List}

Journals

Gábor, A.. G.Qehaja. B (2020). Impact of ICT in Healthcare System and Implementing IT strategy In developing Countries. South East European Journal of Sustainable Development - SEEJSD, Vol 4, 1-11. Retrieved from https://seejsd.unt.edu.mk/ http://conference.unt.edu.mk/

Qehaja, B., Abazi, B., \& Hajrizi, E. (2019). Enterprise Technology Architecture solution for eHealth System and implemantion Strategy. IFAC PapersOnLine, 52(25), 370-375. https://doi.org/10.1016/j.ifacol.2019.12.554

Qehaja, B., \& Hoxha, V. (2018). Principal steps for monitoring real time patients through ICT in developing Countries. IFAC-PapersOnLine, 51(30), 166-170. https://doi.org/10.1016/j.ifacol.2018.11.280

Qehaja, B., Bajraliu, A., Shabani, A., \& Hajrizi, E. (2016). Enterprise Integration, Networking and Virtual Communications. IFAC-PapersOnLine, 49(29), 144-147. https://doi.org/10.1016/j.ifacol.2016.11.090

\section{Conferences}

Jashari, Arton; Qehaja, Besnik; and Berisha, Diellza, "Privacy and Security Data communications issues in HealthCare Ecosystem" (2018). UBT International Conference. 106. https://knowledgecenter.ubt-uni.net/conference/2018/all-events/106

Qehaja, Besnik; Krasniqi, Gazmend; Bajraliu, Ardian; and Shabani, Amet, "Web application penetration testing" (2016). UBT International Conference. 59. https://knowledgecenter.ubt-uni.net/conference/2016/all-events/59

Bula, Edin; Qehaja, Besnik; and Bula, Ines, "Use of semantic web development and linked data in improving e-Health care systems" (2018). UBT International Conference. 116. https://knowledgecenter.ubt-uni.net/conference/2018/all-events/116

Ymeri, Exhevit; Qehaja, Besnik; and Hoxha, Ramiz, "Synchronization and High Availability of Data by Using Technology of SQLServer 2012 Always On" (2017). UBT International Conference. 104.

https://knowledgecenter.ubt-uni.net/conference/2017/all-events/104

Jashari, Xhelal; Vllaço, Edin; Fetaji, Bekim; and Qehaja, Besnik, "Conceptual Design and Software Development of Clinical Management System based on Microservices" (2019). UBT International Conference. 259. https://knowledgecenter.ubtuni.net/conference/2019/events/259

Alidema, Fitim; Vokshi, Valbona; and Qehaja, Besnik, "Experiences of medical staff with ICT in Kosovo Hospitals" (2019). UBT International Conference. 315. https://knowledgecenter.ubtuni.net/conference/2019/events/315 


\section{Appendix 1: Planning Interview Questions and Target Groups}

\subsection{Interview Questions for Medical Doctors}

Purpose: Understanding the work of medical staff, the way of treating patients

Question 1: How often do you check monitored patients (e.g.: once a week, once a month, etc.)?

Question 2: How aware are the patients, in keeping with your recommendations for monitoring their own health status?

Question 3: Which data would be the most useful for you to correctly know the health status of a patient, per patient target group?

Question 4: How would you want the data to be grouped, providing the best information about patient status?

Question 5: From all the data and information that is being collected about a patient, what would be indicators that would trigger an alarm, for an emergency intervention?

Question 6: Do you think such monitoring is unethical?

Question 7: Which department in the hospital does need the most urgent digitization?

Question 8: Do you think digitization would help the patients in terms of their own health awareness?

Question 9: Do you think through a digital health system using mobile devices preventive measures can be taken for non-patients.

\subsection{Interview Questions for Hospital Nurses}

Purpose: Understanding the work needed by nurses and the way of treating patients

Question 1: What type of data filters on the dashboard interface do you want in order to search for specific parameters and/or patient data?

Question 2: How often do you visit chronic patients at home? What data would best help you keep an eye on them? Are they different for patients with chronic diseases and those with just after an acute intervention? 
Question 3: What type of data and statistics would you prefer to have on dashboard to best perform your daily tasks?

Question 4: What type of warnings or alarms (as defined by protocol) would trigger an alarm for you, at which point would you have to get in touch with a doctor for emergency treatment?

Question 5: On a scale from 1 to 10 , how do you think digitalization method for distance monitoring is needed?

Question 6: Do you think the health information system would help reduce your workload?

Question 7: What are the most embarrassing barriers you had to cope with in the last five years? Where do these barriers come from?

\subsection{Interview Questions for Hospital Managers}

Purpose: Understanding the work needs of Doctor-staff and the way of treating patients with health issues

Question 1: What is the average time that patient spend in your hospital?

Question 2: Which type of data would help you to know the current and future status of available staff, (e.g. an urgent treatment can be arranged for patients in need)?

Question 3: What type of data would best help you in order to keep track of availability of internal infrastructure (ward capacity, medical devices, drug inventory etc.) in place?

Question 4: What is the most important data that you would need in order to reduce the general costs of your hospital?

Sub-Question: What data reports would help you long-term planning, including staff, infrastructure, pharmaceutics, etc.?

Question 5: In what forms do patients set up appointments for their treatment?

Question 6: What are the barriers that you had to cope with in the last five years? Where do these barriers come from? 


\subsection{Interview Questions for Patients with Chronic Diseases}

Purpose: Understanding the work needs of Doctor-staff and the way of treating patients with health issues

Question 1: How often do you need to see your doctor for regular checks have you got in the last month?

Question 2: Are you trust to send day/night symptoms to your doctor using the internet and do not go physically to your doctor?

Question 3: Do you think that using a digitized system you would not need to go to the doctor so often?

Question 4: Do you think that the measurement of vital parameters would be able to be performed by a digitized system?

Question 5: Do you think it is easier for you to contact your doctor online? How much would you trust this digital communication?

Question 6: Do you think the digitization would increase the quality of your life?

\subsection{Interview Questions for Patients with Acute Interventions}

Purpose: Understanding the work needs of Doctor-staff and the way of treating patients with health issues

Question 1: How often do you need to see your doctor for regular checkups after surgery?

Question 2: How do you usually get in contact with your doctor?

Question 3: How much do you need to see the vital parameters measured at the doctor after surgery?

Question 4: What vital parameter do you think you should send to your doctor?

Question 5: What data about your current status do you think a doctor will need?

Question 6: How much would you believe or trust in such a system?

Question 7: In case of house treatment after an acute surgery, would you accept any monitoring equipment? 
Question 8 : At what point do you feel the digital monitoring cannot give a suitable solution for your problem?

\subsection{Interview Questions for Healthy People}

Purpose: Understanding the work needs of Doctor-staff and the way of treating patients with health issues

Question 1: During fitness exercises what parameters you want to measure?

Question 2: Do you accept to keep any kind of sensor that measures your activity and how do you trust them?

Question 3: Which vital parameter do you measure more often?

Question 4: If you feel uncomfortable with your health sometimes, would you be able to measure vital parameters and send it to the doctor?

Question 5: If you accept to share vital information with a doctor or a hospital how do you feel about security and privacy?

Question 6: Do you think such digitization would increase your quality of life? If yes, in what aspects?

\subsection{Interview Questions for Information System Expert}

Purpose: Opinion on the general overview of this system architecture

Question 1: What is your opinion about the architecture approach in general? Is there interoperability between the layers?

Question 2: Do you think the proposed architecture is safe to end-to-end communications?

Question 3: What is your opinion about the safety standards of the three architecture levels?

Question 4: Do you think that the proposed system can be implemented in a real-world scenario?

Question 5: Do you think in Kosovo the real-time monitoring as a strategy component is necessary and meets the minimum requirements for the Digital Kosovo? 


\subsection{Interview Questions for Software Architect Expert}

Purpose: Opinion on the Application Layer of the Real-time Patient Monitoring System

Question 1: Do you think that the proposed architecture based on Web and mobile access has the potential to support the different type of data that is coming from end user devices, like sensors, smart watches etc.?

Question 2: Do you think that the proposed architecture is efficient for the emergency alarms, can it react in time for the patients and doctors?

Question 3: What do you think about the middleware layer between the app and data?

Question 4: Do you think web-based and mobile technology can use the same database?

Question 5: What is your opinion on the Web and mobile application with the static system that is foreseen to have a direct API for real-time analysis?

Question 6: Do you think that the proposed application architecture correlates with the two layers: Data and Technology?

\subsection{Interview Questions for Database Expert}

Purpose: Opinion on the Database layer of this system architecture

Question 1: Do you think that the connecting different databases by using Watson Analytics would create meaningful data reports that make sense for decision makers, like doctors, managers and nurses?

Question 2: Do you think that the data architecture based on SOA services, would create a decent way of filtering data for all the applications based on Web and mobile access?

Question 3: What do you think about virtualization of services? Do you think it is the right solution?

Question 4: What is your opinion on interconnecting the two different data stores: data from in-house patients and data from end user monitoring devices?

Question 5: Do you think that the suggested database technology is encouraged for the platform based on Web and mobile? 


\subsection{Interview Questions for Telecommunication Expert}

Purpose: Opinion on the Telecommunication layer of this system architecture

Question 1: Do you think the proposed technology of communication is appropriate for different data with different sensors or technology?

Question 2: What is your opinion on the proposed network solution, can it reliably support real-time communications?

Question 3: What do you think about virtualization of services? Do you think this is the right solution?

Question 4: What is your opinion on the proposed network redundancy?

Question 5: What is your opinion on communication and how secure is this solution?

Question 6: Do you think the Network Architecture meets the requirements for an eHealth system?

\subsection{Interview Questions for Cyber Security Expert}

Purpose: Opinion on the Security of this system architecture

Question 1: How would you ensure the data integrity?

Question 2: What would you suggest to ensure the confidentiality of data?

Question 3: Is the proposed remote access setup secure enough?

Question 4: How would you ensure the best possible availability of the eHealth architecture?

Question 5: What security solutions would you suggest to keep end-users secure? 


\section{Appendix 2: Summary of the interviews}

\subsection{Preparations}

In the thesis quality research methods has been applied. As it was mentioned earlier I used semi-structured interviews. The reason why I chose this method has to do with the nature of my research field that how technology helps health services. In general, interviews are more effective for qualitative research, enabling to gather a wealth of information from different groups of interested people about: attributes, behaviors, preferences, feelings, attitudes, opinions in the context of health services digitization.

The empirical research has been conducted in several phases: preparation of the interview, interview phase, transcription phase, stage of data entry into the paper.

a) Prior to conducting the interviews, the interviewers were personally contacted by phone and email, and each of them agreed to conduct the interview together, also agreed to audio interview except the two "Acute group" patients who did not accept the interview, but they agreed to answer questions in writing. Participants were asked for their consent and signature, they participated on voluntary basis and whenever there is a conjecture they can be withdrawn. On the same page where the participants have signed the informed consent, it was explained to them that their privacy and anonymity will remain secret. The questionnaire was prepared in both English and Albanian, and all participants in the interview process requested that the interview be made in Albanian, I translated them in English for my research.

b) During the interviews phase most of the interviews were conducted in regional hospitals with medical staff and with some patients. Whereas patients with chronic and acute diseases were interviewed at home due to their health status, the healthy patients were interviewed at universities and in fitness saloons, in different cities (see the table below).

c) The interviews' time has varied around 25 minutes per interviewees. The table 2 . below shows the number of respondents and all the basic information for interviewees. Because of the privacy of each interviewer, an ID has been assigned. Identification shows only the type of target group profile and the ordinal number 
(DR01 Doctor, NU01 Nurse, MA01 Manager, CH01 Patient Chronic, AC01 Patient Acute, HP01 Healthy People, and EX1 Expert ICT. IDs assigned to each interviewee are also presented in this table and they will be used throughout this thesis. For data interpretation, the groups are divided into three domains. Domain A medical personnel, Domain B presents patients, and Domain C represents information systems experts and computer Scientists.

\begin{tabular}{|c|c|c|c|c|c|}
\hline $\begin{array}{l}\text { Type } \\
\text { of } \\
\text { group }\end{array}$ & Domain & Profile & Location & $\begin{array}{l}\text { Number of } \\
\text { Interviews }\end{array}$ & $\begin{array}{l}\text { Format of } \\
\text { the } \\
\text { interview }\end{array}$ \\
\hline DR & A & $\begin{array}{l}\text { Cardiologists } \\
\text { Specialists of } \\
\text { Cardiologists } \\
\text { Emergency care } \\
\text { Medicine Specialist } \\
\text { of Cardiac } \\
\text { physiologists }\end{array}$ & $\begin{array}{l}\text { University Clinic Prishtina, } \\
\text { Gjilan, Peja, Ferizaj, Prizren } \\
\text { and Kamenica }\end{array}$ & 8 & $\begin{array}{l}\text { Face-to-face } \\
\text { Voice- } \\
\text { recorded }\end{array}$ \\
\hline NU & A & Nurse & $\begin{array}{l}\text { University Clinic Prishtina, } \\
\text { Gjilan, Peja, Ferizaj, Prizren, } \\
\text { and Kamenica }\end{array}$ & 7 & $\begin{array}{l}\text { Face-to-face } \\
\text { Voice- } \\
\text { recorded }\end{array}$ \\
\hline MA & A & $\begin{array}{l}\text { Director/Manager } \\
\text { Hospital }\end{array}$ & $\begin{array}{l}\text { University Clinic Prishtina, } \\
\text { Gjilan, Peja, Ferizaj, Prizren, } \\
\text { and Kamenica }\end{array}$ & 6 & $\begin{array}{l}\text { Face-to-face } \\
\text { Voice-records }\end{array}$ \\
\hline $\mathrm{AC}$ & B & Patient Acute & $\begin{array}{l}\text { University Clinic Prishtina, } \\
\text { Gjilan, Peja, Ferizaj, Prizren, } \\
\text { and Kamenica }\end{array}$ & 8 & $\begin{array}{l}\text { Face-to-face } \\
\text { Voice-records } \\
2 \text { Writing }\end{array}$ \\
\hline
\end{tabular}




\begin{tabular}{|c|c|c|c|c|c|}
\hline $\mathbf{C H}$ & $\mathrm{B}$ & Patient Chronic & $\begin{array}{l}\text { University Clinic Prishtina, } \\
\text { Gjilan, Peja, Ferizaj, Prizren, } \\
\text { and Kamenica }\end{array}$ & 7 & $\begin{array}{l}\text { Face-to-face } \\
\text { Voice-records }\end{array}$ \\
\hline HP & B & Healthy People & $\begin{array}{l}\text { University Clinic Prishtina, } \\
\text { Gjilan, Peja, Ferizaj, Prizren, } \\
\text { and Kamenica, Fitness } \\
\text { Saloons, Schools, } \\
\text { Universities }\end{array}$ & 10 & $\begin{array}{l}\text { Face-to-face } \\
\text { Voice-records }\end{array}$ \\
\hline EX & $\mathrm{C}$ & $\begin{array}{l}\text { Senior cyber security } \\
\text { Engineer Mercedes } \\
\text { Benz } \\
\text { DusseldorfGermany } \\
\text { Database Expert } \\
\text { Software Developer } \\
\text { Outsource developer } \\
\text { IS Expert } \\
\text { University Professor }\end{array}$ & $\begin{array}{l}\text { EU Mission Kosovo } \\
\text { Expert ICT Kosovo. }\end{array}$ & 5 & $\begin{array}{l}\text { Face-to-face } \\
\text { Voice-records } \\
\text { and writing }\end{array}$ \\
\hline
\end{tabular}

Table 2. Interview groups

\subsection{Analysis}

\section{Medical Personnel (Domain A)}

The purpose of collecting data through personal interviews was to better understand the existing working conditions of doctors, nurses and administrators, then, through interview questions, to learn about their views about digitalization in medical services, to collect specific requirements from the point of view of their particular role, and finally get some information about the readiness to accept the digital system. The answers are categorized 
according to each interview question and are represented in percentage of responses. In the end a comprehensive recommendation is given.

\subsubsection{Doctors}

In the framework of interviews with the medical staff, interviews were conducted with 8 doctors in four areas: Cardiology, Specialists of Cardiologists, Emergency Care, and Medicine Specialist of Cardio physiologists in the regions of Prishtina, Gjilan, Peja, Ferizaj, Prizren and Kamenica. During the interview, all interviewees expressed high willingness to cooperate and their sincerity was found in all responses. Interviews were conducted during working hours in order to closely monitor the status of health system status at clinics. In some cases, the interview lasted longer, such in case of the emergency doctor at the Gjilan / Gnjilane center (DR03), due to his obligations to handle multiple emergencies. As an exception, the interview with the doctor in the Prishtina region (DR01), due to his obligations, was conducted in the early hours of the morning, before the official working hours, although he answered the interview questions but gave very short answers that have not been well elaborated.

Taking into account the feedback of doctors regarding the question about the frequency of checking their monitored patients, the results are as follows: $60 \%$ of them state that they check acute and chronic patients once a week, while $40 \%$ think that their patients are being checked every 1 month, but all respondents confirm that depending on their pathology and complexity, acute and chronic patients may have more frequent visits to doctors up to 2 times a week. Whereas consultations for the third group of patients (healthy people) are rarer because the majority consult by phone.

Regarding how much they adhere to doctor's recommendations while monitoring their health, $70 \%$ of respondents think that patients' awareness is not satisfactory enough, because patients often hide their data from health staff regarding their physical activity, nutrition, also in some cases complying with the prescribed therapy. Patients along with hiding the data often go unprepared to doctors who do not access the preliminary medical file of their earlier visits or treatments they have had in the past. While $30 \%$ of doctors highlight the risk to their patients, 
especially for those who are chronically ill. These patients respect the given recommendations, they are in aware of their medical records and during the doctor's check they take all the medical reports of the previous treatments.

To the question of what the most useful data for doctors are to accurately understand the health status of the target groups of patients, $80 \%$ of them claim that all vital parameter data is needed, such as heart rate, blood pressure, blood sugar levels, blood fat levels, and breathing. While $20 \%$ state that in addition to vital parameters, it is necessary also additional lab data.

Whereas, how doctors want the data to be grouped, providing the most valuable patient status information, $90 \%$ of respondents say they would like to group this data into lab data and imaging data, while $10 \%$ of them state that it is necessary to include radiological data in addition to the laboratory data and imaging data.

Being referred to the answers to the question of how data or information would be indicative of an alert for emergency patient treatment, all respondents' state that any vital parameter data that exceeds its normal value is an alarm for us and the patient should be treated with urgency. However, as far as they are concerned the values of the parameters vary depending on the age, condition and health problem of the patient.

While on the question of whether the current traditional patient monitoring is functional, $100 \%$ of doctors state that the current monitoring is not very functional. Owing to the lack of accurate patient data they cannot provide accurate patient diagnosis and accurate health monitoring.

As matter of fact, $40 \%$ of respondents think that a patient's medical history would probably be needed for accurate patient monitoring and treatment, possibly even from their birth.

In the question of which hospital ward type has the most urgent need for digitization, $80 \%$ of respondents state that the emergency department is more than necessary, while $20 \%$ of them state that cardiac surgery and neurosurgery are the units that need urgent digitization However, according to all respondents, a digitization of the health system is more than necessary in all wards and at all levels. 
Regarding the connection between digitalization of the health system and patients' awareness, $70 \%$ of respondents state that this affects a lot in patient awareness, while $30 \%$ of them state that it is up to age because often the elderly people do not have good skills in using technology.

On the question of whether the digital system affects the use of mobile phones, applying preventive measures among healthy people, all respondents state that this affects a lot because thanks to the use of this system and mobile devices they have prior information on their condition health and their vital parameters. According to the respondents, if they have access to the "healthy people" data in advance, it is valuable information for them to make accurate decisions for further preventive measures.

When asked how they evaluate this research and how this research would help the health system, all respondents emphasized that this is a very necessary and well-sought research, while $15 \%$ of them state that based on their experience that they earned in Western countries digitalization of the healthcare system is a successful practice there.

\subsubsection{Nurses}

Interviews with the nurses were also conducted in the clinics where they work in the region of Prishtina, Gjilan, Peja, Ferizaj, Prizren and Kamenica. 7 nurses were interviewed who provided high co-operation during the interview and answered all questions correctly, except for the interview with the head of the Ferizaj / Urosevac region (NU01), who due to her fatigue as a result of the night shift, she has partially answered the interview questions, but all the answers she has given have provided some valuable information.

To the question of how often they visit chronic patients at home, and what data would help to keep patients undergoing with chronic illnesses, $90 \%$ of respondents state that patients are not visited at home, only in rare cases when it is necessary. While $10 \%$ of them state that there are cases when they visit patients at home according to doctor's instructions and depending on the patient's need and condition. All respondents state that data that would help keep patient under surveillance is personal patient details, patient treatment methods, and patient's key parameters such as blood pressure, heart rate, blood sugar, as well as data on contagious diseases and allergies. 
While questioning what kind of patient data filters nurses will need to have available on the panel interface, $70 \%$ of respondents from the nursing group state that vital parameter data is very important, data for the compliance with therapy, as well as data on patient nutrition and physical activity, because these are the key data to have a more accurate picture of the condition of the patient. Meanwhile, $20 \%$ of respondents state that in addition to vital parameter data, they also need personal data as well as other data for the status record they have made during previous visits. The vast majority of respondents' state that in addition to the above data, it is necessary to have information on the amount and timing of the medication.

According to all respondents it is necessary to have information on each treatment and visit of the patient, also in hospitals and other clinics, because as far as they are concerned there are cases when they treat the patients and they do not know about their earlier treatments, and the lack of accurate information may adversely affect the delivery of patient therapy.

When asked what kind of data and statistics are available in day-to-day nursing work to perform better, $65 \%$ of interviewed respondents state that data on patient number and visits, data on patient health history, and statistics on physical activity are some of the data that would help them perform better. $35 \%$ of respondents state that it is necessary to have data and statistics on the number of patients, the amount and timing of medications, medical devices, preliminary diagnosis data as well as data and statistics for clinic beds. According to all respondents the success of their performance depends on proper communication with the doctor.

When asked which kind of patient information would trigger an alert for them and at which point they would need to contact doctor for an emergency treatment, $90 \%$ of interviewed respondents state that raising any value of vital parameters that exceeds the usual patient average is evidenced as alarm, while $10 \%$ of them state it depends on the illness, age and condition of the patient.

Whereas, concerning the question on the scale 1 to 10 , how they think that a digitalization method for distance monitoring for home patients is needed, all respondents state that this method is highly needed and evaluated with maximum points 10 , because it enables them to 
monitor the condition of the patients they treat. In the question of whether the information system will help reduce their workload, all respondents state that digitalization of the healthcare system would greatly affect the reduction of workload as they think that processes are digitized and have more information. $90 \%$ of them state that this would help relieve patient management, identify the amount of medication and medical devices, while $10 \%$ of them think this will also help in searching for medical records for patients since they often encounter delays until a medical file is requested. According to the respondents, the digitalization of the health system may pose a challenge for older nurses, who often find themselves in difficulties using technology.

Obviously taking into account the answers to the question: "Which are the barriers that nurses have been coping within the last five years and where do these barriers come from?" $60 \%$ of respondents state that the lack of an Information system that can identification of visits and services offered to patients, those are among the main barrier, while $40 \%$ of respondents state that the main barriers are related to the lack of a fast system that will enable identification of available medicines, lack of beds for patient placement and treatment.

\subsubsection{Managerial Staff}

Interviews with Hospital Director/Manager were conducted in 6 hospitals in the regions of Prishtinë, Gjilan , Peja, Ferizaj, Prizren and Kamenica, where all interviews were conducted in a good spirit of cooperation, where their sincerity was found in the responses in general. One case was with the manager at the Prizren clinic (MA02), who due to his age expressed pessimism in the answers that he provided.

What is the average time spent on patients in the hospital where they work, from hospital admissions until treatment ends, $60 \%$ of them state that the average time is $20-30$ minute, $20 \%$ declare that is 30 minutes, while $20 \%$ of them state it depends on the patient's problems and condition. While questioning which staff statistics would help improve the provided medical services, all respondents state that the exact number of staff within a daily, weekly and monthly schedule, as well as the statistics of the number of patients treated by each health staff. $20 \%$ of them state that in addition to the number of staff and patients it is necessary to have statistics about the use and possession of beds and medical devices. 
To the question of what kind of data would help managers to be informed of the current and future status of available staff in order to have a better staffing arrangement and better and faster treatment, $70 \%$ of respondents state that data on patient waiting time, staff load on service delivery, number of patients treated, termination, types of patient disease, as well as data for scheduling terms for patients are the necessary data for them to help you improve a more efficient management. $30 \%$ of respondents declare it is more than necessary to have data on location and time of health staff during working hours, as well as data on more frequent diagnoses of patients treated by healthcare staff.

Regarding the question of what are the most important data that are needed for managers who would help them reduce overall hospital spending, $60 \%$ of respondents state that there are data on patient visits and times, as well as data on the state of the drug stock. Meanwhile, $40 \%$ of respondents state that the number of staff in proportion to the number of patients treated, as this helps in further planning, as well as to gather data as much as possible regarding the medical expenditures and consumption of medicines at the hospital.

When asked whether these data would help with longer-term planning, including any part of the health system environment, all respondents state that these data will help a lot in longterm planning, where $40 \%$ respondents state that the hospitals they work have a planning system based on the data of the previous month. While the question as to what form patients are setting for their treatment, $60 \%$ of respondents state that for acute patients the time is set in advance by the doctor himself during the last visit of the patient, however in the case of the other patients there is no prior term because all checks are made on the day of the visit. Whereas, $40 \%$ state that the appointments are scheduled only in the case of pre-determined examinations and radiological examinations and recordings, these appointments are determined by the medical technical staff based on the doctor's recommendations. All respondents state that the term is made in physical form.

Regarding the barriers faced by managers over the last five years, $60 \%$ of respondent's state that the main barriers concern the dysfunction of a unique primary, secondary and tertiary system in the Kosovo healthcare system for managing health staff data, as well as data on patient treatment. Meanwhile, $40 \%$ of respondent's state that among the main barriers is the 
lack of planning of medication stock and medical devices and lack of direct communication with suppliers, as it requires multiple approval for supply with medical devices and medications.

\section{Patients (Domain B)}

\subsubsection{Acute patients}

8 interviews with acute patients were conducted in the region of Prishtina, Gjilan, Peja, Ferizaj, Prizren and Kamenica, all interviews were conducted either in hospital clinics or in the home. Despite of their state of health they offered great cooperation during the interviews and tried to provide answers based on their experiences as acute patients. In the case of patient AC01 in the Gjilan region, who was hospitalized in Gjilan, where due to his older age and poor health, his family assisted to answer the questions? However, the patient, despite the serious health condition from cardiovascular problems, has offered high cooperation and sincerity in response. It is worth noting that the patient (AC02) in Kamenic agreed to be interviewed in the presence of the family 5 days after the operation and has been one of the most interesting interviews.

In the second domain the questions are compiled in order to gain a view of the current treatment and how they are able to receive a digital health treatment in terms of monitoring their health. Below all the answers are grouped by interview questions and presented in percentage as a group.

Regarding the need for regular doctor visits and examinations after surgery, $40 \%$ of acute patient respondents stated that they need control every 2 weeks. $60 \%$ of them state that they need to have checkups and doctor visits every month.

Whereas, regarding the contact with the doctor, $80 \%$ of them state that they have contact with the doctor during their visit to the hospital, while $20 \%$ declare that they have to contact also by telephone for consultation, in case of health condition deterioration, because according to them it is problematic to set the time for control due to the huge workload of health staff. 
To the question of how many times a day they need to see the vital parameters measured at the doctor after the operation, $90 \%$ of the respondents state they need to measure vital parameters at least once a day, while $10 \%$ declare that they need measure up to 2 times a day. Regarding the need for which vital parameters is needed to be measured in order to control their health status, $80 \%$ of them state that it is necessary to measure blood tension and heartbeat, while $20 \%$ of them claim to need measure the amount of blood sugar, , and measure and monitor the calories and diet of the food. As for the question of which data they think a physician would need, $75 \%$ of them say heartbeat and tension are needed to be sent to the doctor, while $25 \%$ declare that they have to send calories, diet, and physical activity.

Regarding the trust of a remote communication with their doctor, $80 \%$ of respondents declare that they would trust a digital system that enables distance communication with their doctor because, in emergency cases a short consultation already may help a lot. Regarding the possibility of placing any monitoring device in patients, all respondents declare that they are willing to set up such devices that monitor their health, but $65 \%$ of them state that they need assistance from their family members or other persons to put these devices in their body.

\subsubsection{Chronic Patients}

7 interviews with chronic patients were conducted in the region of Prishtina, Gjilan, Peja, Ferizaj, Prizren and Kamenica, where 4 of them were interviewed in their homes, while the other 3 patients were interviewed in hospital clinics. Interviews with chronic patients were conducted in a good spirit of collaboration, where they answered all questions based on their needs and experiences as chronic patients. This was the case with the patient in the Prishtinë region $\mathrm{CH} 01$, the interview was conducted during the time she performed the tests at a hospital clinic in Pristine, with the recommendation and guidance of a physician. Whereas, with the patient $\mathrm{ACH} 02$ in the Ferizaj region, the interview was conducted while he was waiting for his routine appointment with his cardiologist at the hospital clinic in Ferizaj.

When chronic patients were asked about how many treatments they had during the last month, $60 \%$ reported having 2 visits during the last month, 10\% said they had a visit during the last month and $30 \%$ of them declare that they have visits only every 3 months. Answering the next question, as what symptoms they think should be reported to their doctor using the 
internet instead of visiting in person, 70\% answered if would be necessary to send their symptoms, blood pressure, heart beats and blood sugar, they would do it, while $30 \%$ declare that it is necessary to send the results of fat and breathing, too.

If a digital system would minimize the personal doctor visit, all respondents declare that they are in and they believe that such a digital system enables them not to go so often to doctors. $80 \%$ of the respondents claim they often need to communicate with the doctor for quick consultations, and in these cases they use phone communication with the doctor.

90\% of respondents agreed with the measurement of vital parameters can be done by themselves, with a digitized system, assuming the technology will be advanced enough, only $10 \%$ of them think that this could happen in the near future.

Asking if digitalized connection to the doctor (via Internet) is easier for them and they trust in the solution, all respondents say it's easier to digitize their contact with doctors, and they believe in this digitization. $70 \%$ of respondents state that digitalization of connection with the doctor would help reduce their waiting time, as well as enable continuous communication. All respondents declare also such digitization would increase their quality of life, where $30 \%$ of them think this would affect the doctor's correct diagnosis and treatment, while $70 \%$ of them think that this would have an impact on improving their health by monitoring vital parameters. According to the respondents in most cases, due to the lack of continuous monitoring, their life is often jeopardized because the they reached an alarming value of any vital parameter but they had serious difficulties contacting the responsive healthcare staff.

\subsubsection{Healthy People}

Interviews were conducted with healthy people in the region of Prishtina, Gjilan, Peja, Ferizaj, Prizren and Kamenica, 4 of which were conducted in university schools and 6 during their fitness exercises. All interviewees were willing to cooperate, except for the one concerned by Peja municipality (HP07) who was a nutrition expert and he was more discreet in providing answers.

When asked about when doing exercises in fitness what parameters they want to measure, 75\% say they want to measure their physical activity, heartbeat and calories they consume 
and spend, while $10 \%$ do not want to measure heartbeat but just physical activity and calories. The latter mentioned group think that constant heartbeat measurement can cause stress, they do not want to know at all the times what is their condition. $25 \%$ of respondents want to have data on fats and blood pressure.

$80 \%$ of respondents declare that they would accept to carry any type of sensor that measures their activity and they trust inn them, because monitoring their own activity at any time they feel more secure about their health. While $20 \%$ declare that they do not like to keep any kind of sensor that measures their activity, but they are willing to use them if they have any health concerns.

As for the question of which life parameters are most often measured, everyone says that heartbeats and blood pressure are the parameters they measure, while $10 \%$ claim to measure oxygen saturation, as well. Regarding whether they want to send their own vital parameters to doctors, $90 \%$ state that they want to send vital parameter data to doctors, while $10 \%$ declare that they do not want to do it. All respondents believe that ongoing monitoring of their activity and their life parameters would help increasing their health awareness and, if it is necessary, would help doctors determine their diagnosis as accurately as possible.

Regarding the security and privacy of data when distributing vital information to a doctor or hospital, $65 \%$ claim they are worried about their data exposure but believe in the security of advanced systems, which should be supported by law. $35 \%$ believes the security and privacy of their data are not compromised, however physical data based on paper may be unsafe in the hands of healthcare staff.

\section{Experts (Domain C)}

The purpose of collecting data through personal interviews with computer scientists and information systems experts to provide additional contribution to the dissertation in order to gain a better view and assessment of the architecture of the proposed information system. A secondary objective is to justify the proposed architecture. The following assessments are divided into the level of architecture proposed at the application level, data, communication, security, and information system. 


\subsubsection{Application architect}

M. Shamolli is a software engineering assistant at UBT, with 7 years of experience in software development, and has completed BSc and MSc in computer science. Additional information that he provided is of added value to my application architecture proposal. The interview was conducted in the laboratories of the respective university.

To the question that the recommended Web-based and Mobile-based architecture can transmit data from devices such as smartwatch, sensor, etc. Shamolli thinks it's possible but it depends very much on what standard of communication has been chosen and is there a middleware between the devices and the web application. It is assumed that the solution using API in Mobile and Web he thinks there will be no problem, but care must be taken with the solutions of communication technologies between the MobileApp protocols and the receiving device. From his perspective, it is a promising architecture since SOA architecture provides many opportunities for cases for eHealth information systems, which are mostly web-based and micro-based.

When asked in what extent is possible to represent medical data in Web applications and Mobile, he believes that with the recommended solution it is possible. He thinks that ASP.net technology is the right choice but recommends that it should be carefully selected, since many options and types are available, like CORE, MODEL, and MVS etc.

Shamolli recommends that role-access should be set at the application level, since not every application should have access to the same services, but in terms of communication this is only possible during the development of these applications where roles are of great importance. This recommendation also applies to the Watson analytic link for the reports that need to have roles for each user. It also seen as a good opportunity because such a system will represent the data in how healthcare should see them to be understandable by the staff.

To the question of whether we can use the same database for mobile application, the expert says yes, as it's just a different platform but communication is the same as in Web access.

To the question of whether this architecture can provide real-time data, he thinks it can, but depends heavily on communication infrastructure. He also confirms that real-time 
communication is possible on the application level of architecture if it developed in the way as it is recommended in the strategy document.

To the question of whether virtualization of applications is the right choice, he thinks it is an adequate solution and does not hurt the performance of applications, since application virtualization is recommended to be separated from data virtualization.

\subsubsection{Database (Data Science) Expert}

E. Imeri is a Database Administrator with 17 years of work experience at EULEX Kosovo. He implemented the database security solution entirely at the European Commission. . He has a BSc and MSc in Computer Science with Data Science specialization in Kosovo. During the interview, he was very professional and gave enough suggestions regarding this research, especially in terms of data security. There has been little reservation about conversations about the job he does, due to legal obligations. The interview was conducted in a restaurant because we could not meet at his office due to the strict rules of the Eulex Mission in Kosovo.

Concerning the multi-source based statistical system solution, meaning communicating with different databases in order to extract valuable information, Imeri confirms that the solution is adequate but the configuration should be done professionally because the requirements can be different or combined and require professional configuration and fit the type of medical data stored on the proposed databases. IBM Watson is a platform that provides interface that adapts to the nature of the health system, so he thinks it's the right solution and the cost also is acceptable compared to the value of the work it does.

Microsoft-based SOA architecture, whether it's right selection to work on Web and Mobile platforms, or adapt to the database architecture that's recommended, he agrees that for the time being most of today's architecture work installed in this form and they are very addicted to web and mobile applications. As far as data integrity is concerned, it is a challenge to the level of networks and standards used at the access level. Indeed, he believes that if the application arrives with the information from the end device via the application level to the saved database and the data format is no problem, then it is the responsibility of developers how to preserve the attributes and the data entity. 
Regarding appropriateness of data virtualization, he argues that the solution offered is adequate; the only suggestion is that applications and data should be divided into separate hardware because of the amplifier. It is recommended to be incorporated within the private cloud.

Imeri agrees with encryption through AllwaysOn. It is the right solution, as even communication between applications and databases within the same institution should be encrypted even though they are within the same infrastructure.

\subsubsection{Network communication expert}

A. Salihu is a Network Engineering Assistant who earned a BSc and MSc in University of Pittsburgh in USA and currently he is a Phd student at TU Vienna.Salihu is a telecommunications engineer with experience in TDM / ATM / IP mixed wireless Backhauls for global telecom carriers. He is an expert in automated network configuration, service orchestration and AWS cloud services. Very enthusiastic and determined to design, deploy and optimize practical communication systems. He is currently working on $5 \mathrm{G}$ implementation. During the interview, he was very cooperative and enthusiastic about the proposal of this research, where he gave sincere suggestions and meaningful recommendations. The interview was conducted at a restaurant and lasted slightly longer than the other interviews.

Network resolution for the entire topology as it is recommended for the Real-time Patient Monitoring System, he noted that the healthcare data processing requires an adequate solution starting with the end user where MQQT protocol have been chosen. He believes this is the right solution because it allows communicating with standardized passwords while the data format that is transmitted belongs to the application level should be presented on web or mobile applications. In terms of communication it is an adequate solution. He confirmed this technology supports real-time communication, as well, all solutions starting from 4G network is adequate but he also recommends, it requires coverage when sensor data is received. As far as the WAN network for stakeholders is concerned, there is a redundancy, but the aspects of VPN tunnels should be taken into consideration, whether it is created only for communication of health data or for all traffic. He thinks it should be separated as it 
creates loads in the broadcast, even though fast devices have been planned. He thinks service vitality is the right solution, but virtualization at the network level is also good solution, as it makes possible the scalability in performance. At the level of network aggregation, he believes setting it redundant is acceptable.

Considering the value network security, Salihu thinks that starting with user access the recommended HTTPS satisfactory, at WAN level, 4G has built-in security, WiFi at home or at institutions is controversial enough, but it is important to filter traffic with Mac-Address at least for the user who uses medical data. PKI Certificate is a necessary solution as it is planned and he believes that at most levels an acceptable security is planned in this network topology.

Salihu agreed, the proposed network is realistic, it is rational as it is seen in the solutions offered and the topology can be simulated with virtual software before it is built and but it is good to look at costs at all levels.

\subsubsection{Security expert}

A. Bajraliu is a senior security consultant engineer at Mercedes-Benz in Düsseldorf, Germany and holds a Bachelor of Computer Science degree from UBT University. He has 6 years of experience in security and telecommunications and he is also certified at all levels at Cisco. Bajraliu initially received the proposal material and then the interview was conducted via Skype. During the interview, Bajraliu expressed high readiness for cooperation and responded honestly to all questions. After the interview on Skype, he also emailed me in writing all recommendations and responses. Bajraliu has been approached once again for additional consultations on written questions and he cooperated and responded very candidly to the additional recommendations.

Concerning maintenance of data integrity Bajraliu agrees that with the proposed network architecture data integrity has a good degree of protection; using HTTPS protocol with TLS encryption and SSL in broadcast integrity is protected. He warns to be careful with data modification in different database and/or communication between them. Regarding communication between hospitals and the main center he draws the attention to potential 
risks of secure WAN network and suggest a VPN tunnel for data traffic between stakeholders, as well as using these two IPsec protocols suffice for such a network. The main argument is the solution in question does not allow overloading the network to avoid a problem in the broadcast performance since the fact that each packet must be encrypted at the output side and decrypted at the input side requires significant networking device performance. But with an appropriate method of configuration minimizing the unnecessary traffic he believes this solution might be acceptable. He mentions another problem, access level between the ISP and the datacenter setting up a firewall is a good idea as it can prevent attacks from outside such as DoS, fishing, etc.

To the question of how you think about, is this solution acceptable: Bajraliu commented the end-user security issues, too. According to his opinion, Two Factor Authentication and Certificate ensure the expected level of security, he recommends using mobile msg or a combination of the serial number of the phone and message to confirm accessing the system.

\subsubsection{IS expert}

B. Zylfiu has a PhD in Information Systems in Kosovo, is a professor at the State University in Pristina. Zylifu during the interview has been as open as possible to any level of architecture proposed, and provided very detailed answers, trying to explain all his answers and recommendations as simply and clearly as possible. The interview lasted longer than other experts and was contacted once again via Skype interview for additional response.

During the interview with Information System expert, in their view the first question was how cross-platform architecture is interfering. Zylifu concluded that the recommendation of web and mobile platforms today are more adaptive and allow users to interoperate with minimal hardware resource usage. SOA-based architecture creates good opportunities to communicate with different databases, but it should be seen if the user modifies the information in a database, this change whether go across the architecture of other databases. He acknowledged that the solution offered to a statistical system based on real-time communication, which is Watson Analytic, is an adequate solution since such a system in Kosovo is a challenge to build. If it can be related to the data and application architecture, he 
believes it is a well-targeted solution based on best practices. We know that IBM has developed many successful products that help doctors. He added, this solution is worthwhile in terms of cost.

Zylifu thinks that the security is dealt with at every level, and especially the private cloud aspect is an adequate solution since services are more closely for any kind of changes for future requirements While other security aspects did not need much more efforts, he is afraid of creating loads on the technological devices as they have to deal with encrypting and decrypting of any packet is a burden to technological architecture.

Regarding standards, he recommends following the general technological standards, paying special attention to the medical standards, especially in case of medical devices, In the future they would be more adaptive to the existing infrastructure.

Zylifu finally commented the need for eHealth strategy. He mentioned he previously participated in several state-initiated projects such as digitization of schools, but the strategy for the Health Information System is more than necessary because with the information that we have been working for six years still has not been able to functionalize the existing system. This is the reason why there are much more in planning than in realization, often the same project gets multiple funding; nevertheless, we still have functional systems in most hospitals in Kosovo.

\subsection{Evaluation}

\section{Medical Personnel (Domain A)}

Based on interviews with medical staff, we see high level of readiness to use ICT in their daily work. The difficulties of entering patient records and information and other activities in the healthcare system hampers hospitals and blocks productivity in decisions making and in the daily work of health personnel, including the treatment of patients, management processes, personnel communication, and work planning.

It turns out that the lack of data creates problems to the health staff when treating patients, as they often encounter concealment of data from patients either consciously or unconsciously. As a result of this problem they convincingly admit that the digital information system for 
them is a very effective option for handling this ferry group (acute and chronic patients) as well as other groups. According to doctors, the better treatment of patients would enable patient health monitoring in real time and on a longer period. Health monitoring in real time and on longer periods helps health staff in making the most accurate diagnostic and therapeutic decisions Digitalization of the health system is necessary at all levels of health service in Kosovo, as well as incorporating other stakeholders: pharmacies, health insurance companies etc. This way each stakeholder could have the necessary information of patients.

\section{Patients (Domain B)}

According to the results of interviews with patients, they need constant contact with healthcare staff. They suffer from disadvantages contacting healthcare staff when they need it, because of difficult social and geographical conditions. As a result, they contact the health staff only during their regular checkups, or when the health condition is worsening, this way contacting other consultants in case of need is almost impossible.

The results show that patients view the digitalization of the health system as a good opportunity it enables almost continuous communication with the health staff and eliminates long waiting time in hospitals therefore increased care is expected. Patients believe in digital system, although short consultations on telephone with the staff have been productive but at the same time, they believe that a more advanced digital system would enable them a more meaningful communication and monitoring. The elderly interviewed patients although it appears that they support the digitalization of the health system, there is a lack of use of technology, based on the results it appears that they need assistance from family members or a second person in the use of ICT during their treatment.

The results of the interviews by healthy people show that this group supports their health monitoring through ICT, because they see it as an opportunity to raise awareness of health maintenance, but at the same time, they express reservations monitoring their health all the time, because they feel pressured about the data they provide. 


\section{Experts (Domain C)}

According to the results of the interviews with the experts, depending on their field of expertise they see the proposed architecture realistic in accordance with the requirements expressed by target groups and other potential users.

The Web and Mobile platform-based application architecture is seen by experts as the right solution for user access to the information system, as it does not require multiple hardware resources. According to expert recommendations, the functioning of patient monitoring in real-time depends also on the selected device that communicates with the application. Experts see as an adequate solution he choice of micro-based SOA architecture platform measuring vital values, which enables communication between databases and applications, when it comes to real-life communication between patients and healthcare staff. They also recommend that role sharing in access to services is vital for the better health system function. Experts support the data architecture layer, in harmony with the Watson statistical analysis system; it greatly helps the availability of data for all stakeholders, especially target ferry groups and healthcare staff.

Experts point to the need for increased care in data retention and privacy, where a solution can be the duplication of encryption, however it may reduce the performance of devices and communication platforms. Regarding selected data storage technologies, experts see effective the virtualization of services and the statistical support system.

The findings from experts about the communication level recommend that the selected communication meets the criteria for a real-time end-to-end communication, but also recommend that the selected way of encryption on one side protect security and data privacy, but on the other hand may lower the performance of the devices.

Security experts pointed out the communication architecture in general looks reliable at all levels, both in user access and datacenter, but they also recommend more detailed access to WiFi access as Homecare Access.

Regarding the results about the information system in general, experts concluded that architecture has a linkage between layers and also has the capacity to respond to health 
system requirements. In a comprehensive context, experts support architecture and consider it responsive to stakeholder requirements.

\section{Triangulation of results}

Based on the conclusions from Medical Staff (Domain A) Patient (Domain B) and Expert (Domain C) we see a correlation between each Domain. Domain A and Domain B requests support from Domain C. In general, it is seen that patients accept ICT as a solution, while healthcare staff sees ICT as an opportunity to improve the performance of the health system as well as increasing the availability of data and information. On the other hand, experts

estimate that the proposed architecture and strategy supports the demands of every interested groups. 


\section{Appendix 3: Technology Architecture Repository}

\subsection{Network WAN and LAN architecture}

In the technological architecture, the proposed network is comprised of the central area as the core point of the "Core Cisco Router" network, located in the central facility of the Ministry of Health in a special network in the center of Pristina. The Core Routers contain four routers that route all of the internal traffic and are interconnected with "edge" routers, through the firewall, which are also located within the same object. The vast majority of Routers are at Cisco Company and support many protocols in terms of performance security and endurance.

The border routers enable two-way traffic with network providers outside the facility. Data center communication is performed through a "Multilayer Switch" within the Ministry facility and supports the HTTPS Load Balancer for data security and balancing requests from users using web services to connect to the database and link layers hierarchical "core", "access" and data.

In the WAN network communication protocols we have used two types of protocols that are: Border Gateway Protocol (BGP), Enhanced Interior Gateway Routing Protocol (EIGRP)

EIGRP was used as an internal communication protocol. The reason for using EIGRP is that it offers Load Balancing, Security, classless IP addressing, and uses bandwidth algorithm, so the bandwidth line that is faster routes the traffic from that line. As well as the protocols between the Internet Service Provider and the Hot Line Standby Router (HSRP) protocol is a routing protocol that allows routers to use a number of routers that act as a single virtual router, with the failure of one router there is another standby router for the redundancy.

User Distribution. A layer that connects all devices defined as User Access. It is used in all facilities of interest parties that have communication with the health center where there are a large number of end-devices accessing the network, such as interest groups, hospitals, pharmacies, state institutions, patients etc. Communication is done with a data center, where through the endpoints that are located on each side is a Gateway Router that supports VPN to encrypt traffic from user to private cloud services that are physically located in the main 
facilities of the Ministry. Type of connection to the stakeholders and communication is made through fiber optics since post-war state infrastructure is located $1348 \mathrm{~km}$ throughout Kosovo and can be used the same by creating a VPN tunnel only for specific traffic for Communication Health Architecture.

\section{Data Center}

Services in the proposed architecture are located within the WAN network and by building a private cloud where many different services are allowed to avoid large investments and the time required to secure, implement, and replace the IT infrastructure. This method will work by utilizing the virtualization of 3-level services: those of applications and services and operating systems for server infrastructure.

\section{Private Cloud Infrastructure}

Infrastructure as a Service (IaaS): provide resources that are manageable and can grow, depending on the needs of the user. In individual perspective, the cloud offers enormous virtualization opportunities. In this model, the private cloud infrastructure is located in data centers, including space for data storage, servers, hardware parts, and layers for virtualization.

Virtualization allows the creation of many simulated environments with resources dedicated to a single hardware system. Virtualization of applications at the operating system level makes use of the infrastructure and servers in the private cloud, as well as the way how the software is developed today: to be expandable in terms of infrastructure and flexibility of development.

Among the core benefits of virtualization is that in combination with the microcomputerbased software architecture make it easy to extend the application and its infrastructure. There are several types of virtualization techniques that offer similar features. Most applications running on a server can easily share a machine with others if they can be isolated and secure. Moreover, in most cases, different OS are not required on the same server. OS level virtualization systems are designed to provide the isolation and security required to use multiple applications or copies of the same OS 
Platform as a Service (PaaS): Provides a private cloud environment, in which developers can use platform resources to develop applications. These include resources such as operating systems, different programming languages, databases, web server etc. As part of these platforms, various software infrastructure capabilities are provided, including the application platform, the integration platform, the medical analysis platform statistics, streaming video tele monitoring services, back-end services.

Software as a Service (Saas): The main feature of this model is that the service provider takes responsibility for the entire application and manages it. One of the major benefits of this model is that it has lower cost than buying an application. The main goal is to support end-users or simple users (Benharref \& Serhani, 2014).

Data Backup and Storage Space is a possibility to provide a regular backup of data. However, in many cases, backup data must be encrypted and protected from unauthorized access both internally and externally.

\section{Access Layer}

It is among the underlying points in which all end-users access the network and eHealth services. The intent of this layer is the logical separation of network access and often initial filtering (technology like Wireless 802.1X, 4G, 5G, and MQTT sensor-based protocol) (Lloret et al.2017). Based on referencing architectures there are specific terminology that apply to this layer, which are also used in the technical solution provided as:

User Access: Access components that enable end-user connections that use Ethernet devices (PCs, laptops, phones, sensors, tablets), so all users who have Network Interface Cards - NIC. Access is largely based on the Web service, as well as the Mobile Web App using the HTTPS protocol.

SA - Server Access: Access components that provide connection only to stakeholders equipment (hospitals, pharmacies, insurance companies etc.). These include devices and applications that enable continuity of the eHealth system, such as Web servers, file servers, authentication servers, etc. The list also contains exchange of messages through HL7 protocol, Corba, Healthcare Environment Distribution, XML. 


\subsection{Medical standards}

Other standards for data interoperability and health records such as EPSOS (European project demonstrating interoperability between EMRs in Europe), IHE (initiative to improve the interaction of health information) SNOMED (nomenclature for significant information exchange of health information) or The EMA structure (standard for compilation of pharmaceutical information) will be foreseen to be integrated into any system that wants to enable the import and export of healthcare information. ISO / IEC 62304 is an international standard that defines the life cycle requirements for the development of medical software in general and the software incorporated in medical equipment in particular. The standard describes the provisions according to the potential of the software to create a risk that could potentially result in damage. Certification is usually interpreted as meeting the provisions of ISO 13485 that specifies the quality management system that a producer must meet.(Stamate et al., 2018)

\section{Healthcare Environment Distribution (HED)}

HED is a middleware architecture which paves the path for a further collaboration and distribution between end-user applications including inheritance systems. DHE information is represented by the core concepts centered on the entity relationship model (ERM) eg. entities, relationships, and attributes. Indeed this implies that each service relates to one or more entities or the links between the entities and the information being taken are described by the attributes of the entities or relationships involved. DHE supports and fosters at a large extent the cooperation between individuals from different units of different health care centers.

\section{HL7 Communication}

As it is highlighted in the literature, the HL7 protocol in our architecture enables us to communicate between information systems (applications) in hospitals, based on electronic exchanges of data concerning exchanges healthcare-in (EDI). Optimizing HL7 Version 3 
enables us to communicate with various entities that relate to data and messaging elements, and guarantees the clarity and accuracy of information as well as the adaptability of standards on different healthcare platforms. Through HL7, the architecture will use unique object identifiers and allow the control of sending messages on time.(Piho et al., 2015)

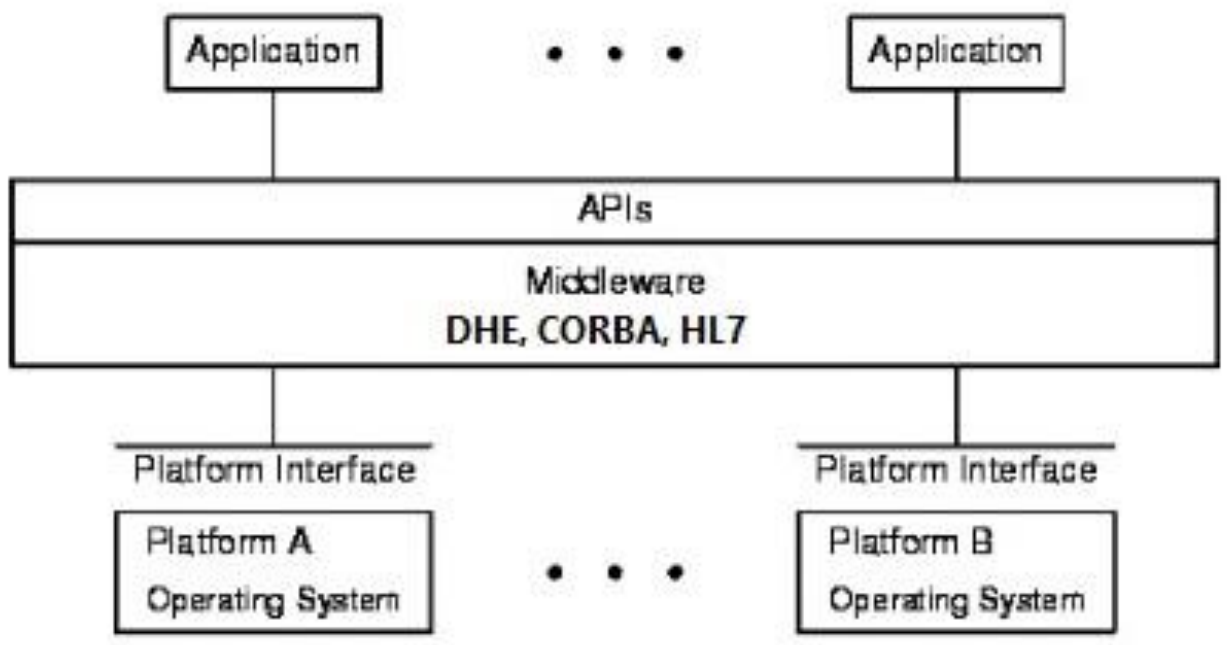

Figure 29. HL7 and DHE function

\subsection{Communication Security}

Protecting the confidentiality of patient information in a common healthcare environment is one of the most complex and demanding tasks. Accurately identifying users, assigning access permits, and complexity becomes even greater when it comes to providing a solution for sharing data between healthcare providers. Traditional approaches such as Mandatory Access Control, Discretionary Access Control and Role-Based Access Control. In the proposed technological architecture, I tried to recommend technologies that support the requirements and do not violate current laws to support privacy and confidentiality.

The solutions that are recommended are primarily based on three main rules such as follows;

- $\quad$ Confidentiality - information must be secure against unauthorized access

- Integrity - information must be secure against unauthorized modification

- Availability - information should be accessible to authorized users 
Communication to hospitals institutions through a VoIP service is the technology that signals voice over the Internet or any other IP network. Via Voice over IP using Internet Protocol (IP) to transmit voice as a packet to an IP network. Through this facility, each hospital center can use IP, Wan Networks and local LAN in hospitals to ensure interaction between doctors and patients at no extra cost. By using this possibility that the existing network offers us, we plan to include, besides the most commonly used communication, a specific channel for voice and video communication using the existing network. The standards to be used are as follows: $\mathrm{H}, 323$ is one of the protocols that enable multimedia messaging services, such as real-time audio, video and IP data, an H.323 terminal can be a PC or a device that can have the H.323 program and multimedia applications that works alongside three other protocols: H.225 / Q.931 Call Signaling, H.225.0 RAS (Registration, Admission and Signaling) and H.245 Media Control using a specific module in the Doctor's and Patient's approach, creating freeof-charge opportunities through this protocol. (Watzlaf, 2011)

SIP is mainly used for Internet conferencing, IP telephony, presence, and instant messaging. So it is a protocol that enables two edges or two extreme points to exchange information (media). It is a client-server-based protocol, in text format and similar to HTTP.As far as security is concerned with LAN and Wan, we have thought that by using specific configurations for voice communication and creating specific VLANs within a physical network, it is enabled to accept ports to perform voice IP traffic from an IP phone. Communication in and out of a network as long as the call arrives at the destination is scheduled to connect where voice over-the-air traffic is better encrypted by using dedicated VPN connections as well as the speed of the VPN process after all encrypted voice and requires decryption at the same time.

\section{Access and network security protocol}

Since the information is not encrypted at the end devices when read by the end user, then the confidentiality of the information is sensitive and is not guaranteed at any time, such as unauthorized access, the security of the message transmission, they will be encrypted by 
using the security layer for the transport layer (TLS). Taking into account each access level, below are all protocols that provide the basis for a secure communication.

The HTTPS protocol is an application layer protocol that is used to transmit all data, even if it is HTML files, image files, results, or whatever. Usually, HTTPS packs on TCP / IP swap and uses SSL to encrypt data on web services.

TCP (Transmission Control Protocol) is one of the major protocols of Internet Protocol Suite. TCP provides reliable distribution of packets or octets between the applications running hosts that communicate over the network. Major web applications such as the World Wide Web, e-mail, remote management, and file transfer rely on TCP.

SSL is a standardized security technology that enables the creation of an encrypted connection between the web server and the browser. SSL allows a server to authenticate itself to an SSL enabled client and, if necessary, allows the client to authenticate themselves to the server. After authenticating and negotiating crypto logical parameters, a secure channel is set so that the client and server can exchange information safely.

The MQTT protocol as an internal communication protocol relies on the medical sensor is proposed in this architecture and is manufactured by IBM and is applied to all IOT devices such as smartwatch, ECG sensor etc. The Message Queue Telemetry Transport (MQTT) protocol is a lightweight protocol suitable for devices with limited processing and memory capacities for the purpose of sending data over low bandwidth networks. MQTT sets three levels of quality service (QoS) for sending messages. This allows messages to be delivered more than once (MQTT is as reliable as TCP), in terms of device power conservation requires minimal power consumption. The protocol can be applied to most standardized medical sensors, smart watches, and medical devices.(Chooruang \& Mangkalakeeree, 2016).

\section{Security in mobile connection}

Each patient will be identified with the help of the national identification number to access the system and will use the security method as it is discussed in the "Access security in the application architecture" section. The mobile application will have an API that communicates 
with standardized sensors and time slots will be directly controlled by the specific API, under the Bluetooth communication protocols. In the same time using MMQT sensor protocol from IBM and this way the wireless communication standards can be incorporated into the mobile platform. Also, the mobile application will have an additional API for direct connection to the Watson analytical system, which in real-time generates statistics and recommends doctor and the patient's notification as well as within the system foresees a group of caregivers to address the problems whenever they happen. At the same time, notification of the problem is automatically sent to caregivers usually consisting of a group of volunteer or patient's relative. For this service an appropriate solution is recommended in "technology architecture" regarding privacy and confidentiality including the right of the user to control the collection and dissemination of personal information. Indeed, this is carried out by making use of mobile app within the healthcare system, doctors and nurses will have easy access to the services that will be provided by the mobile application, which will also assist patients in need of permanent medical care and patients will not necessarily be limited in hospital or institutional health places, instead of this they are free to be monitored at home. The patient is allowed to move freely and live a normal life, of course under medical supervision.

\section{WAN network security}

VPN WAN Network given the sensitivity of medical records, networking devices like Cisco routers are being supported by private VPNs that provide high-speed Internet traffic to the WAN network for any Stakeholders that communicates with medical services. VPN uses the AES algorithm that provides greater security due to the use of longer encryption keys, so use 128,192 bits or 256 bits in length, while 3DES uses a 168 or 112 bits key that greatly affects security.

\section{Firewall}

In terms of traffic filtration, a firewall is proposed to link the key point between the external network and the central infrastructure that is very sensitive and for this purpose, firewalls are used to protect the network from unauthorized access. These firewalls can be of hardware or 
software nature, which implement the security policies. In our network, we have predicted to have two (2) hardware firewalls that will filter traffic to the inbound and outbound network. These two firewalls will be placed on the perimeter of our network, respectively in the connections with the ISPs from whom we receive the service and have communication from stakeholders using the ISP network for connection to the special network of the ministry where the services are located in The main eHealth data center.(Milito, Morrow, Lake, \& Vargheese, 2014).

\section{Public Key Infrastructure (PKI)}

The main public infrastructure is the public coding system and the digital signature service. This system allows the connection between the public key with the proper user identity under the Certification Authority (CA). PKI infrastructure allows certificates to be widely used, including authentication, encryption, and digital recording purposes in different types of applications.

\section{Mobile access security}

Taking into account the problem regarding the patient authentication, it is recommended that applications are added to dual authenticated 2FA security (2 Factor authentication). In dual legalization, a combination of "something I know", such as password and "something you have", can be the phone, the ground, the card, and the third method. As a second authorization technique, it will be recommended as "something you are", which are biometric certifications. Access via an application that is provided to patients for access to the Internet will ensure that the application encrypts the data sent to the GP and ensures that no data is stored on the mobile device, three times, the wrong access blocks access to one hour and contains their privacy rules. Regarding access to web services, through the HTTPS protocol, all services-related requirements are included using the SSL protocol. 


\section{Infrastructure requirements and their functions}

Starting from the perspective of end-users of the health information system, the minimum requirements that the institutions and stakeholders have to have are as follows:

- $\quad$ Each medical staff in all secondary and tertiary primary hospitals using healthcare services should have access to Web services through a PC, Laptop, IPad, with optimum performance of at least $1.5 \mathrm{Ghz} 4 \mathrm{Giga}$ Ram processor and use the Lan network for communication outside the facility.

- Networking devices within institutions such as Router Wireless router switch, PC, Tablet should support voice, video, and message traffic.

- $\quad$ Each of the technological components inside and outside the office should be placed on a monitored IT domain by the Health Service IT, and have certain privileges in the domain.

- $\quad$ Each device must have licenses for any software and operating system.

- $\quad$ Each regional hospital, the outpatient clinic should have a router that is used as a gateway and supports security protocols that connect to the state-based data center using the ISP's external network.

- $\quad$ Each hospital should have wireless access via hub access point routers, but should be separated in a logical sense from inter-state communications as well as from the internal network.

- $\quad$ Each hospital must have a performance 5 Terabyte cache server for local platforms and traffic up to 500 local users that provides data center synchronization with the primary data center since sometimes there may be network interruptions and the data is initially stored on the cache server and then synchronize with the core date center.

- $\quad$ Each patient needs web access from a mobile or desktop device for communication between stakeholders.

- $\quad$ Stakeholders should have access to Web services through a PC, Laptop, iPad, with optimum performance of at least $1.5 \mathrm{Ghz} 4 \mathrm{Giga}$ Ram processor as well as a router connected 
to the private network with the state network, as well as stakeholders should have restricted privileged access by the Ministry of Health and Information System.

- $\quad$ Each hospital should have a group of at least 2 IT representatives for support and maintenance of network equipment and as virtual or real help desks in each Primary, Secondary and Third level hospital.

- Training of medical staff and IT staff and other users should be done in every respect, and is necessary during each change occurring in the system as well as during system use.

- $\quad$ The state network and state network data center must have at least 6 to 8 Cisco 7900 Routers that deliver services to the ISP network as well as support different communication protocols and support the security aspect of VPN IPsec, internal and external protocol EGIRP, OSPF, BGP.

- In the main data center there should be incorporated at least 5 to 6 servers that support online and traffic platforms up to 500,000 users at the same time, and have data storage space of up to 100 terabytes per server and at the same time processing speed At least 7 processors and up to 64 Gigabyte memory.

- $\quad$ Any existing technological equipment that is located in institutions that has optimal performance will be put into operation for communication needs in each institution. 


\subsection{Roadmap all activity}

\begin{tabular}{|c|c|c|c|c|}
\hline vorcion & BA 8EL INE 1.0 & Duration & $2020 / 228$ (2) & 2021141 m \\
\hline 1.0 & Analysis of requirements & $7.0 \mathrm{~d}$ & & \\
\hline 1 & Funotional roquirementt & $0.0 \mathrm{~d}$ & 2 & \\
\hline 3 & Mosting wth staknohoter & $0.0 \mathrm{~d}$ & $\Longrightarrow$ & \\
\hline 4 & 5y cisem Roguliromonte & $0.0 \mathrm{~d}$. & $\longrightarrow$ & \\
\hline 5 & Deslgn of architecture & $8.0 \mathrm{~d}$. & & \\
\hline 8 & Network Design & $20 \mathrm{~d}$ & 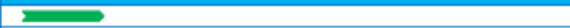 & \\
\hline 7 & Loglo T opology Notwork & $20 \mathrm{~d}$. & 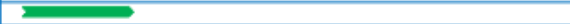 & \\
\hline 8 & Phy closel topolosyy Notwork & $40 \mathrm{~d}$. & 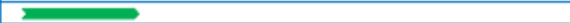 & \\
\hline 8 & Doctign Databs co & $4.0 \mathrm{~d}$ & 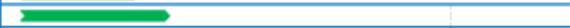 & \\
\hline 10 & Modloling Contro Databsto & $40 \mathrm{~d}$ & 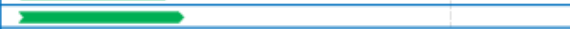 & \\
\hline 11 & Phermsoly Detabste & $40 \mathrm{~d}$ & 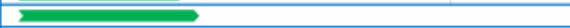 & \\
\hline 12 & Imsao Datastore & $40 \mathrm{~d}$ & 2 & \\
\hline 13 & CIVI Rovic tration Databs is & $40 \mathrm{~d}$ & 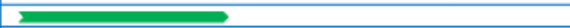 & \\
\hline 14 & Mon itorho Ap plllo attont and Wob do sing & $4.0 \mathrm{~d}$ & 2 & \\
\hline 16 & Dosion oul & $40 \mathrm{~d}$ & 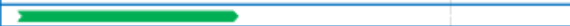 & \\
\hline 18 & Design Middleware & 400 & 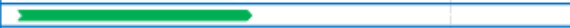 & \\
\hline 17 & \begin{tabular}{|l} 
Docilon Portal Pationt \\
\end{tabular} & $40 \mathrm{~d}$ & 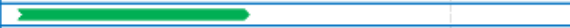 & \\
\hline 18 & Dos lon Doctor Portal & $40 \mathrm{~d}$ & 2 & \\
\hline 18 & Docilon Emoromon corveses & $40 \mathrm{~d}$ & 2 & \\
\hline 20 & Docilon incuranos and PAa ma of portal & $4.0 \mathrm{~d}$ & 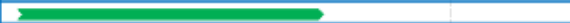 & \\
\hline 21 & Docign Bookond cy ctam & $40 \mathrm{~d}$ & 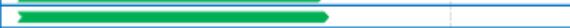 & \\
\hline 22 & Design Monitaring Appilications & 400. & 2 & \\
\hline 23 & Development Phase & $11.0 \mathrm{~d}$ & & \\
\hline 24 & Medloling - sestro Databace & $3.0 \mathrm{~d}$ & $\overline{\underline{I}}$ & $\longrightarrow$ \\
\hline 25 & Pharmacy Databaco & $3.0 \mathrm{~d}$. & $=$ & \\
\hline 28 & Imago Datactoro & $3.0 \mathrm{~d}$ & 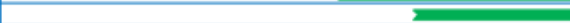 & $\overline{\mathrm{E}}$ \\
\hline 27 & CMill Ragilit tration Data ba co & $80 \mathrm{~d}$. & 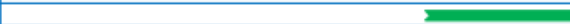 & \\
\hline 28 & Analy to Watc on IEM & $6.0 \mathrm{~d}$. & 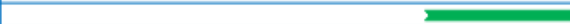 & $\longrightarrow$ \\
\hline 28 & M oblis and Wob Portal Plistorm & $3.0 \mathrm{~d}$. & & 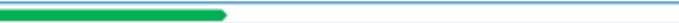 \\
\hline 20 & oul & $3.0 \mathrm{~d}$ & $=$ & \\
\hline 31 & M Idalowaro & $30 \mathrm{~d}$. & & 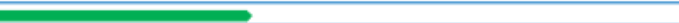 \\
\hline 32 & Portal Pation $W_{\text {W }}$ b & $3.0 \mathrm{~d}$ & & 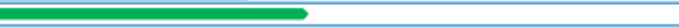 \\
\hline so & Dootor Portal & $30 \mathrm{~d}$ & & 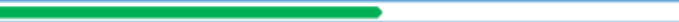 \\
\hline 24 & Emorgonoy corvloosc Portal & $3.0 \mathrm{~d}$. & & 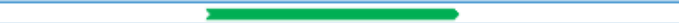 \\
\hline 36 & In euranoo and Pharmasy portal & $3.0 \mathrm{~d}$. & & 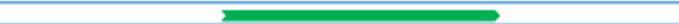 \\
\hline 28 & M on ito ring Appllostione & $30 \mathrm{~d}$. & & $\longrightarrow$ \\
\hline 37 & B sokend fy ctsm web Apploatton & $30 \mathrm{~d}$ & & 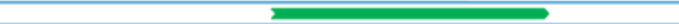 \\
\hline 28 & Notwork WAN & $30 \mathrm{~d}$. & 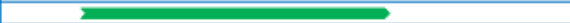 & \\
\hline 30 & Confly Notwork sorvose and soourtity & $3.0 \mathrm{~d}$. & 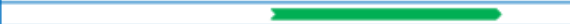 & \\
\hline 40 & Notwork Lan & $3.0 \mathrm{~d}$. & 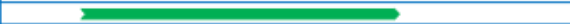 & \\
\hline 41 & Confo Lan cor Voset VoP, IP, Who Aososte & $3.0 \mathrm{~d}$ & ב= & 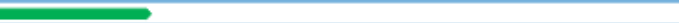 \\
\hline 42 & Privato C bud oonfouration & $30 \mathrm{~d}$ & 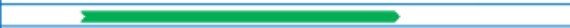 & \\
\hline 43 & Datac ontor corvoos & $3.0 \mathrm{~d}$. & 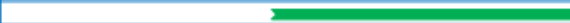 & \\
\hline 44 & Phy closa corver r hetallation & 300 & 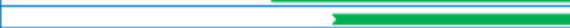 & 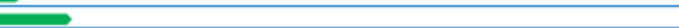 \\
\hline 45 & Virtualization services & $3.0 \mathrm{c}$ & 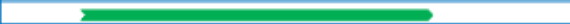 & \\
\hline 48 & Deseter rosoovery & $3.0 \mathrm{c}$ & & \\
\hline 47 & Sosourity Ao osct Conflyuration & $30 \mathrm{~d}$. & & \\
\hline 48 & Conflo HTTPB, TCP, SAL & $30 \mathrm{~d}$ & & 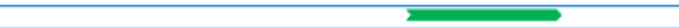 \\
\hline 48 & ODPR Dets & $30 \mathrm{~d}$ & & 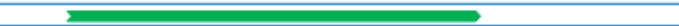 \\
\hline 60 & Standard oHto ath & $30 \mathrm{~d}$. & & 2 \\
\hline 61 & Has thoaro En vriom on t Dictribution & $3.0 \mathrm{~d}$ & $=$ & 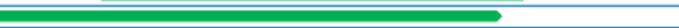 \\
\hline 62 & HL7, HOM & $3.0 \mathrm{c}$ & & \\
\hline 63 & Dotialimsoling & $30 \mathrm{~d}$ & & \\
\hline 64 & Standards implismontation Itoor C 215 & $3.0 \mathrm{~d}$ & & \\
\hline 65 & Ita ndards Impliom on tation IOONEEE 1107 ak & $3.0 \mathrm{~d}$ & & \\
\hline 56 & Secunity Development Phase & $9.0 \mathrm{~d}$. & & \\
\hline 67 & Notwork Wan so ourty & $40 \mathrm{~d}$ & & \\
\hline 68 & Firewall cosourith & $40 \mathrm{~d}$ & & \\
\hline 68 & VPN and P 100 TLS & $6.0 \mathrm{~d}$ & & \\
\hline 60 & in & $600 \mathrm{~d}$ & & \\
\hline 61 & HTTPI Protoool & $6.0 \mathrm{~d}$. & & \\
\hline 62 & Moblis soourity Conflouration & $6.0 \mathrm{~d}$. & & \\
\hline 63 & Publlo Koy intrac truoturo & $6.0 \mathrm{~d}$ & & \\
\hline 64 & Testing Phase & $17.0 \mathrm{~d}$ & & \\
\hline 65 & Web Application Testing & 800. & & \\
\hline 68 & Dostabacos to to ting & $600 \mathrm{~d}$ & & \\
\hline 67 & Notwork Tocting & 400 & & \\
\hline 68 & Soourity To cting & $4.0 \mathrm{~d}$ & & \\
\hline 68 & Dilsettor Rosovery To cting & $20 \mathrm{~d}$ & & \\
\hline 70 & Watcon Analy to Toc ting & $20 \mathrm{~d}$ & & \\
\hline 71 & Ucerc Tocting coosnario & $20 d$. & & \\
\hline 72 & 5y ctam To cting cososnario & $0.0 \mathrm{~d}$. & & \\
\hline 73 & Montoring Phace & 0.01 & & \\
\hline 74 & TAROET LINE FINAL 74 & $0.0 \mathrm{~d}$. & & \\
\hline
\end{tabular}

